\title{
Thermo-reversible Gelation Of Rod-Coil and Coil-Rod-Coil Molecules based on Perylene Diimides
}

\author{
by \\ Elianne Dahan \\ A thesis submitted to the Faculty of Graduate and Postdoctoral Affairs in partial fulfillment of \\ the requirements for the degree of \\ Doctor of Philosophy \\ in \\ Chemistry \\ Carleton University \\ Ottawa, Ontario \\ (C) 2013, Elianne Dahan
}




\section{Abstract}

Polymer gelation is a route to obtain highly organized morphologies. The aim of my thesis was to study the physical gelation of covalently attached perylene diimide (PDI) polymers during their self-assembly. With the gelators used, chain entanglement and network formation trap the solvent causing gelation. Hence, forces such as hydrogen bonding are not a requirement. We first describe the thermo-reversible gelation of PDMS via the physical route, without any functionalization. We discuss a solvent system, propylamine or hexylamine, gelled by PDMS, without any filler, catalysts or chemical crosslinks. The simple route to the physical gelation of PDMS was then extended to preparing PDMS attached PDI based gels. Morphological studies showed that of mono-substituted PDI (Mono-PDMS) gels consist of hollow spheres while disubstituted PDI (Di-PDMS) gels consist of ribbons that are folded along the length into an eaves trough. We attributed this gelation to the entanglement of the PDMS, in addition to the $\pi$ interaction mediated self-assembly of the perylene segments. We then continued our work with PDI attached to a water-soluble side chain; here we studied the gelation of PDI, substituted with Jeffamine ${ }^{\circledR}$ on one imide nitrogen (MJ-PTCDI) or both (DJ-PTCDI). As with the Di-PDMS and Mono-PDMS, the DJ-PTCDI is a Gemini surfactant and MJ-PTCDI is an inverse macromolecular surfactant. Previous studies in our group showed that self-assembly of the PDI was seen in water and aqueous solvent mixtures, but not in the other non-aqueous solvents. In the present case, the morphology of the gels depended on the amount of solvent: non-solvent ratio. Morphological studies showed that although fibers are not seen for DJ-PTCDI gels, the "chaining" of the spheres seems to be present. The SEM image of the cross section of the MJPTCDI sample shows the honeycomb morphology similar to that of DJ-PTCDI. Thus with MJPTCDI based gels also, the worm-like vesicular morphology occurs. Formation of worm-like 
micelles was reported recently, during the association of Gemini surfactants. For our final gelator, we attached oligostyrene to both sides of the PDI (PS-PTCDI-PS), to form a coil-rodcoil molecule, and studied its gelation behavior. This differs from the case of coil-rod-coil molecules discussed before; with Di-PDMS, the $\mathrm{T}_{\mathrm{g}}$ of the PDMS segment is $-125^{\circ} \mathrm{C}$. With DJPTCDI, considering that the glass transition temperatures of PEO and PPO are -50 and $-75^{\circ} \mathrm{C}$, respectively, the $T_{g}$ of Jeffamine ${ }^{\circledR}$ can be expected to be about $-60{ }^{\circ} \mathrm{C}$. However, here the oligostyrene segment has a $\mathrm{T}_{\mathrm{g}}$ of $43{ }^{\circ} \mathrm{C}$. Further, while the rod segment is a large aromatic chromophore, and the oligostyrene coil has aromatic side groups. The unique combination of a flexible polymer and a rigid, extended, $\pi$-electron system lead to interesting morphologies. When cyclohexane and toluene were used as the solvents, spherical morphologies were obtained; while fiber like morphology was obtained when trans-decalin was used. We finally used the PSPTCDI-PS as a guest in its corresponding polystyrene (PS) polymer matrix, in an attempt to make composite films. We showed that by attaching a side group (oligostyrene) which is compatible with the PS polymer, the mixing would be better. Hence, molecularly dispersed composite film could be obtained. Here, experimental factors, such as concentration of the PSPTCDI-PS guest molecules and solvents selection, played a role in obtaining uniform films. The aim was to fabricate films with no crystallization or phase separation. Further research on these composite polymer films will shed light on their outstanding optical properties. 


\section{Acknowledgements}

I am very grateful to have an opportunity to do my Ph.D. under the supervision of Professor P. R. Sundararajan. I appreciate his guidance and continuous support.

This work was supported by the Natural Sciences and Engineering Research Council of Canada

(NSERC). I thank the Ontario Graduate Scholarship for Science and Technology (OGSST) for four years of financial support.

I express my sincere thanks to Mr. Ann-Fook Yang and Ms. Denise Chabot (Agriculture Canada Research Laboratories, Ottawa, Canada) for the E-SEM and confocal microscopy images; Mr J.J Wang for technical support in SEM and TEM. Finally, I thank Dr. Jules Thibault for the Rheology experiments at the University of Ottawa.

I would like to thank my friend Anita Chan, and my colleagues Dr. Kamal Khan, and Dr. Rafiq Islam for their help and friendly discussions.

I especially appreciate my family for their continued love and support.

Most importantly I thank God. 


\section{Table of Contents}

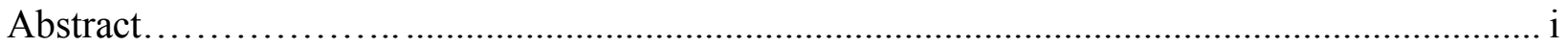

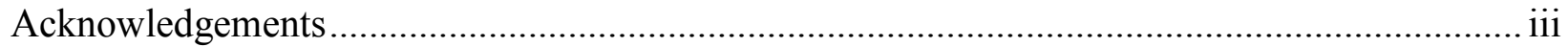

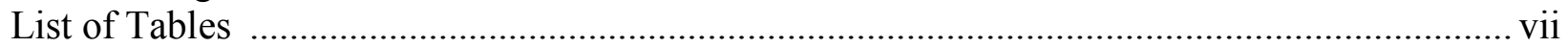

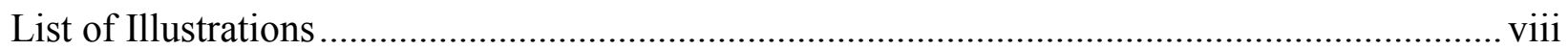

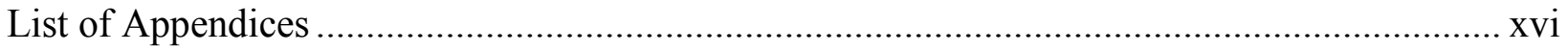

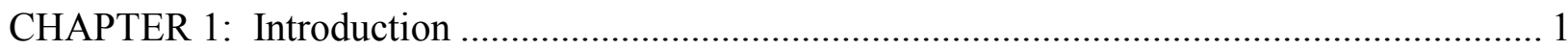

1.1 Basic Principles of Self-Assembly ..................................................................................... 2

1.2 Perylene Diimides: Building Blocks for Functional Supramolecular Architectures .... 4

1.2.1 The Optical Properties of Perylene Diimides in Solution ........................................... 7

1.2.2 Crystalline State of Perylene Diimides ........................................................................... 9

1.3 Organogels vs. Polymer Gels.................................................................................................... 12

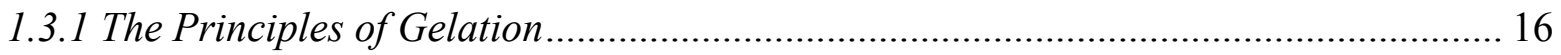

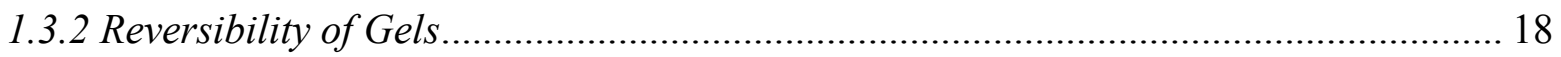

1.4 Organogels Based on Perylene Diimide Dyes .................................................................... 19

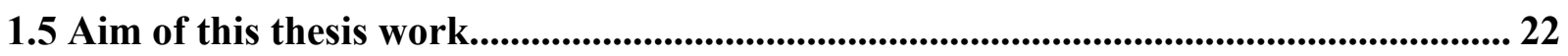

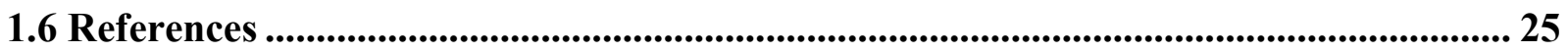

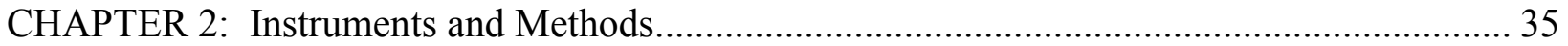

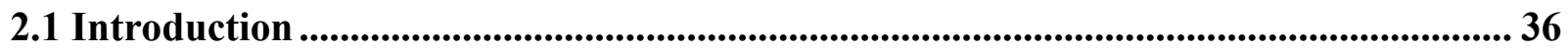

2.2 Instruments and Methods...................................................................................................... 36

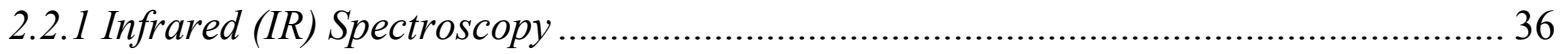

2.2.2 Nuclear Magnetic Resonance Spectroscopy (NMR) ................................................ 40

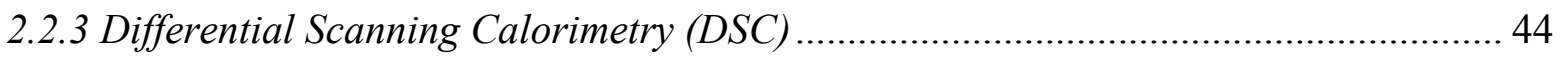

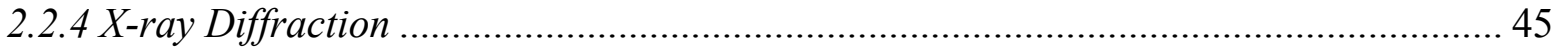

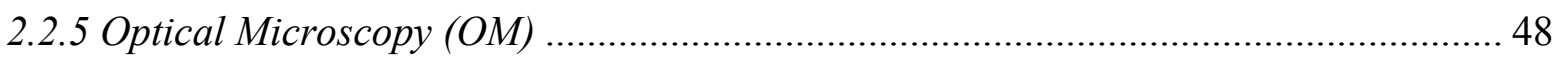

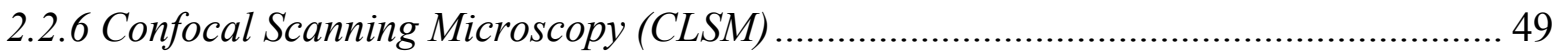

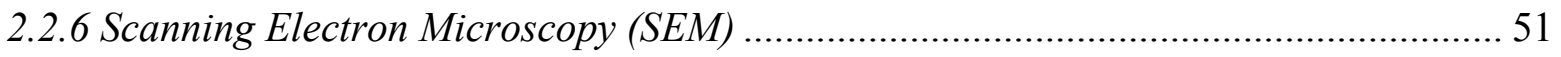

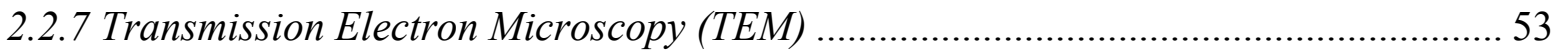

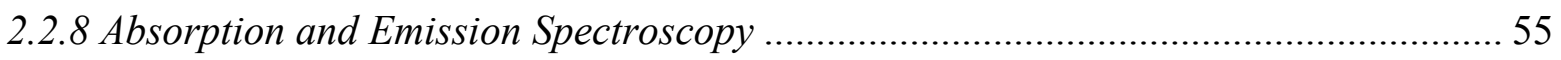

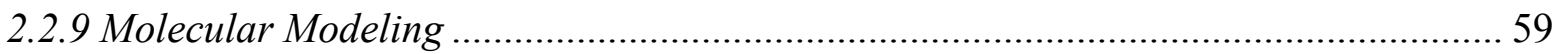

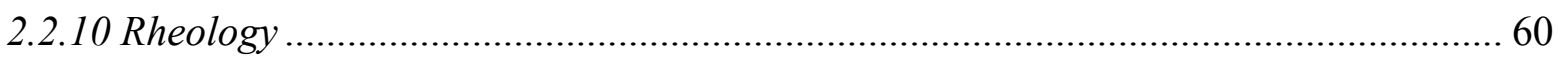

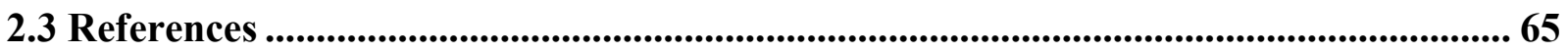

CHAPTER 3: Thermo-Reversible Physical Gels of Poly (Dimethylsiloxane) without Crosslinks

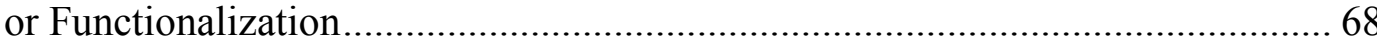

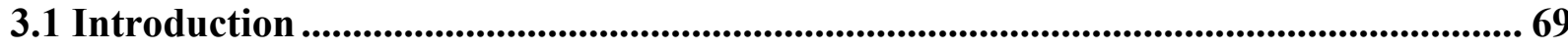

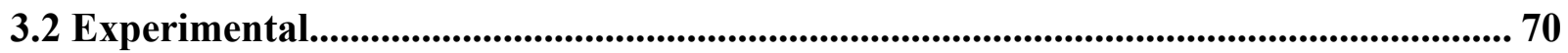

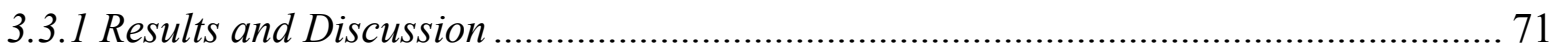

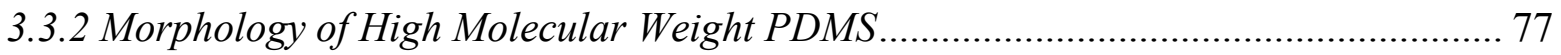

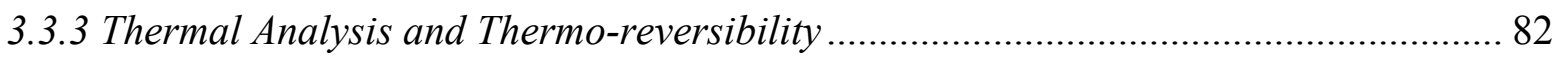




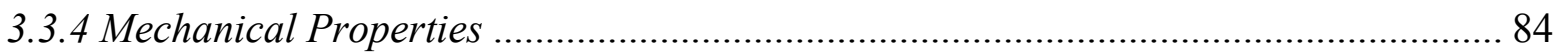

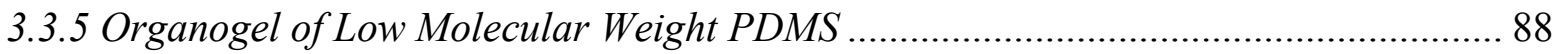

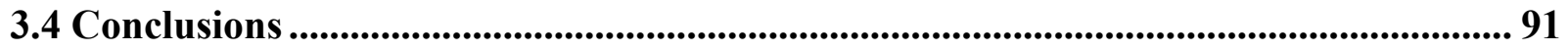

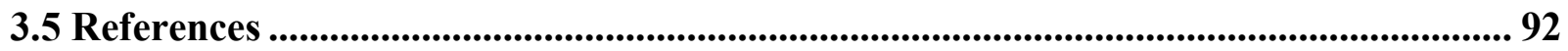

CHAPTER 4: Thermo-reversible Gelation of Rod-Coil and Coil-Rod-Coil Molecules Based on Poly (Dimethyl Siloxane) and PDI without Hydrogen Bonding Groups .............. 98

4.1 Introduction ................................................................................................................................ 99

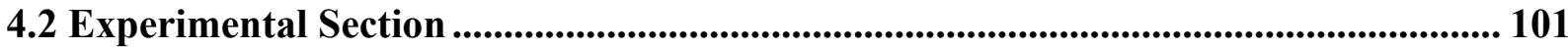

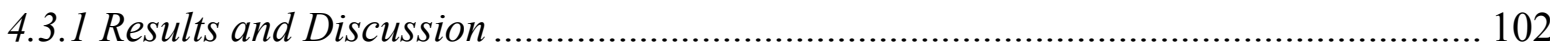

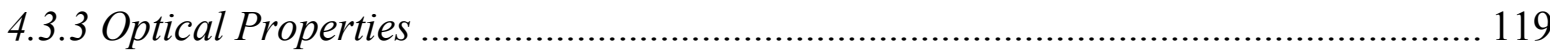

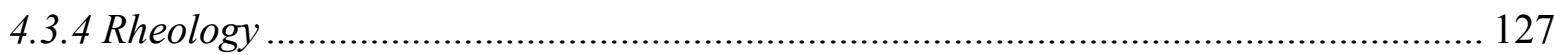

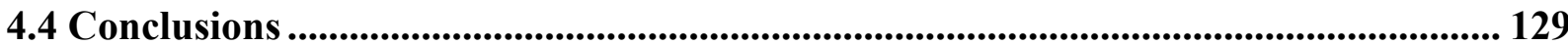

4.5 References ................................................................................................................................... 130

CHAPTER 5: The Thermo-reversible Gelation of PTCDI Mono/Di- Substituted with Ethylene Oxide/ Propylene Oxide (Jeffamine ${ }^{\circledR}$ ) Co-oligomer ......................................... 135

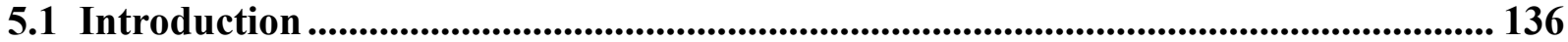

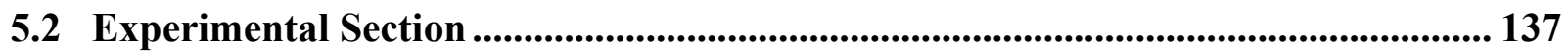

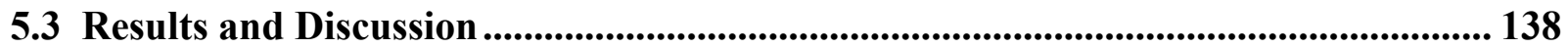

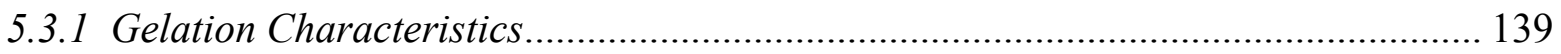

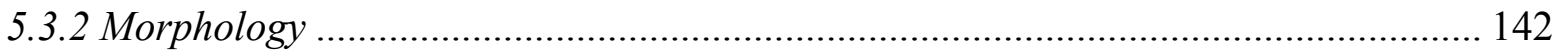

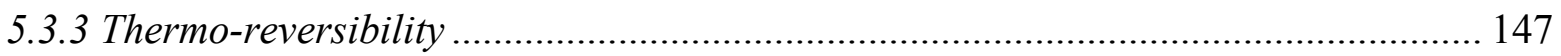

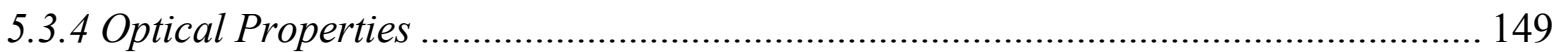

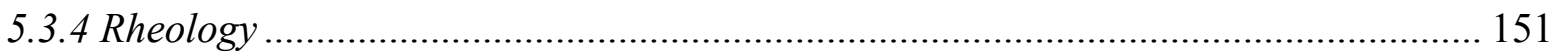

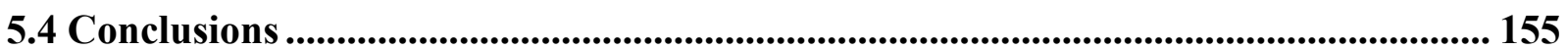

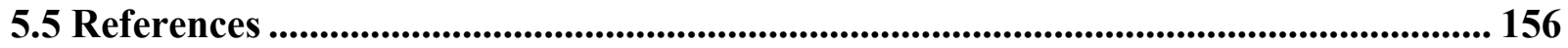

CHAPTER 6: Gelation of Oligostyrene Functionalized Perylene Diimide................................ 160

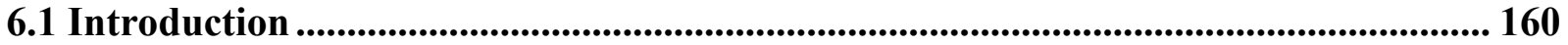

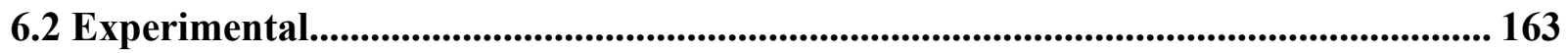

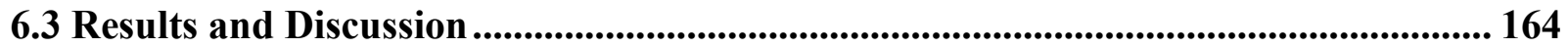

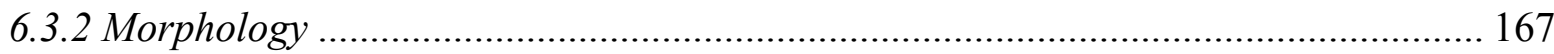

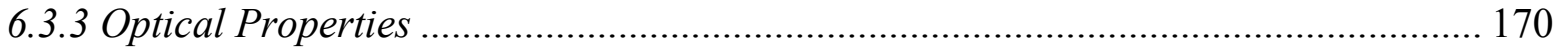

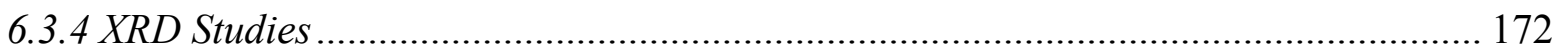

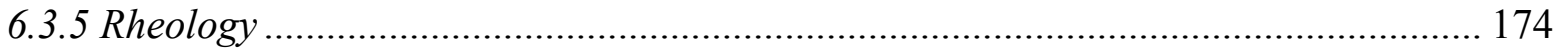

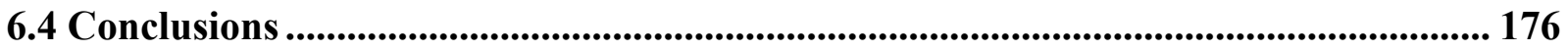

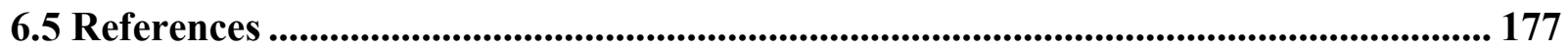

CHAPTER 7: The Fabrication of Composite Films of Self-Assembled PS-PTCDI-PS in a

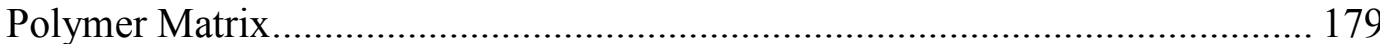

7.1 Introduction ......................................................................................................................... 180 
7.2 Experimental............................................................................................................................. 182

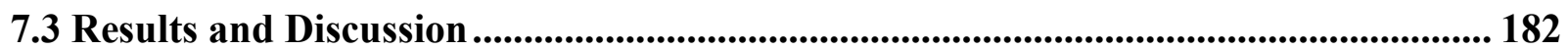

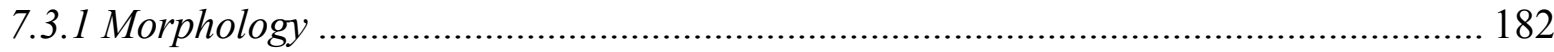

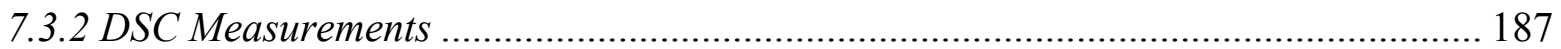

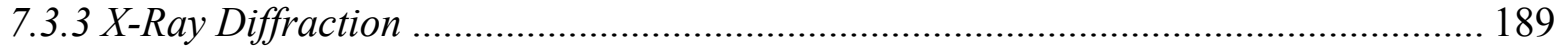

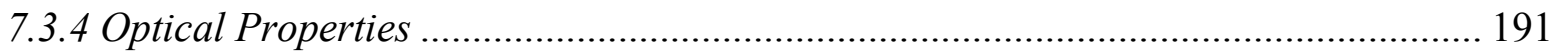

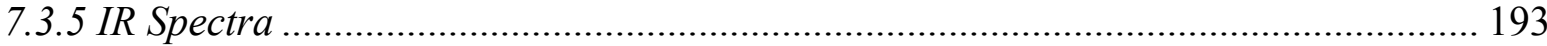

7.4 Conclusions and Future Directions .......................................................................... 196

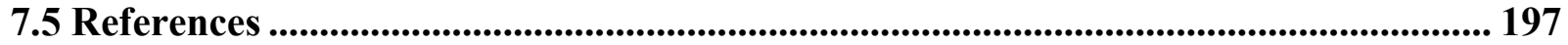

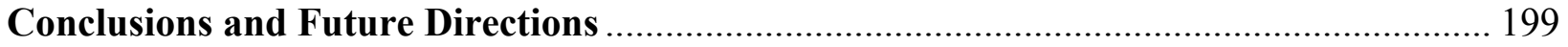

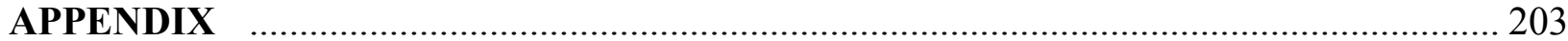




\section{List of Tables}

Table 3.1 The effect of various solvents on the poly (dimethyl siloxane)

sample.

Table 4.1 Variation of heat of fusion of perylene crystals in xerogels of Di-PDMS with

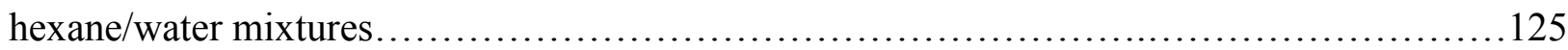




\section{List of Figures}

\section{CHAPTER 1}

Figure 1.1 $\pi-\pi$ stacking of perylene diimides involving (a) longitudinal and (b) transverse

offsets.

Figure 1.2 Schematic representation of a) the pseudo network of LMOG; b) a chemically crosslinked network of polymers; c) entangled physical network of polymers.....................13

Figure 1.3 Formation of a gel is tested by "stable-to-inversion" of a vial......................18

Figure 1.4 Schematic illustration of the formation of viscous lyotropic fluids and gels from

PBI 1 in methylene chloride and acetone.

\section{CHAPTER 2}

Figure 2.1 Group frequency and fingerprint regions of the mid-infrared spectrum..............38

Figure 2.2 The spin- $1 / 2$ nuclei include the most common ${ }^{1} \mathrm{H}$ NMR nucleus, as well as many other nuclei such as ${ }^{13} \mathrm{C},{ }^{15} \mathrm{~N}$ and ${ }^{31} \mathrm{P}$.

Figure 2.3 An NMR spectrum can be plotted as a function of frequency (a); however, it is best to use the chemical shift scale (b) in which frequencies are expressed relative to that of an agreed reference, such as TMS in the case of proton spectra

Figure 2.4 NMR spectrum acquired by varying the magnetic field over a small range while observing the RF signal from the sample. .42

Figure 2.5 Schematic representation of DSC cell. .44

Figure 2.6 Bragg construction illustrating the principle of diffraction where $d$ is the spacing between two atomic planes. .45

Figure 2.7 Schematic of the Bragg diffraction with the powder X-ray diffractometer. 
Figure 2.8 In a CLSM, with image processing, many slices can be superimposed giving an extended focus image.

Figure 2.9 Schematic diagram of a Scanning Electron Microscope........................51

Figure 2.10 Partial Jablonski diagram for Absorption, Fluorescence, and Phosphorescence....54

Figure 2.11 Emission spectrum as a mirror image of the absorption spectrum...............55

Figure 2.12 Steady state (a) flow behavior (b) viscosity of Newtonian and non-Newtonian fluids. 61

Figure 2.13 AntonPaar MCR 502 Series Rheometer with PP25 Parallel Plates.... .62

\section{CHAPTER 3}

Figure 3.1 Images of PDMS in Various Solvents. .72

Figure 3.2 PDMS gel (at right) prepared with propylamine as the solvent. The flow of PDMS starting material is seen in the vial on the left. .73

Figure 3.3 Images of inverted vials containing PDMS gel and xerogel....................73

Figure 3.4 ${ }^{1} \mathrm{HNMR}$ spectra of (a) PDMS gel (b) bulk PDMS in the siloxane region.............74 Figure 3.5 FTIR spectra of PDMS/propylamine gel, xerogel, PDMS/hexane solution and bulk PDMS

Figure 3.6 (a) Optical micrograph of PDMS gel from propylamine. (b) OM of the xerogel (c) Three dimensional image using confocal laser microscope (d) E-SEM image recorded at $-140^{\circ} \mathrm{C}$; (e) TEM micrograph of the xerogel; (f) Sample kept at gelation temperature for 30 minutes and cooled. .78

Figure 3.7 (a) Illustration of the formation of network and spherical domains during gelation with PDMS: (i) chains in bulk; (ii) chains dissolve and expand on heating; (iii) upon cooling, 
chains start collapsing and forming network trapping the solvent leading to gelation; (b) E-SEM image of a fracture domain showing the fibrils between domains...........................79

Figure 3.8 X-ray diffraction traces of PDMS gel (top) and xerogel..........................8 80

Figure 3.9 DSC traces of gels of PDMS during the heating, cooling and second heating cycles. The sample was kept for 45 minutes at $45^{\circ} \mathrm{C}$ during the cooling cycle to enhance the gel

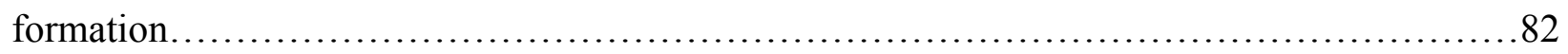

Figure 3.10 Optical micrographs of (a) gel at room temperature (b) at $50{ }^{\circ} \mathrm{C}$; (c) at $65{ }^{\circ} \mathrm{C}$

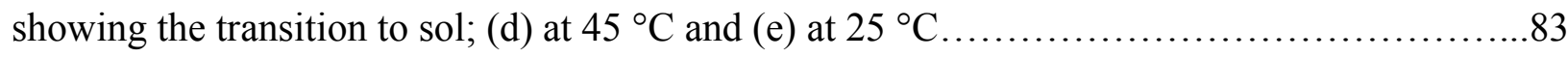
Figure 3.11 (a) Variation of G' and G" with frequency for PDMS gel, xerogel and the bulk PDMS (b) Thermo-reversibility of G' of the PDMS gel.

Figure 3.12 Stretching of (a) original PDMS high molecular weight sample and (b) the gel. (c) photograph of the manual stretcher used. .86

Figure 3.13 Compression and relaxation of (left) the original high Mw bulk PDMS and (right) the gel. The top photos show the compressed sample and the bottom, after withdrawal of the piston. Note that the gel recovered from the compressed state.

Figure 3.14 (a) Optical micrograph of the gel with low molecular weight PDMS (b) formation of rods during the organo gelation of low Mw PDMS. .88

Figure 3.15 DSC trace of low Mw PDMS during the heating, cooling and second heating cycles. The sample was kept for 45 minutes at $45^{\circ} \mathrm{C}$ during the cooling cycle to enhance the gel formation .89 


\section{CHAPTER 4}

Figure 4.1 Solution and Gelation Temperatures of (a) Di-PDMS (b) Mono-PDMS ( $\left.\mathrm{M}_{\mathrm{w}}: 1500\right)$.

The insets show the inverted vials containing the gels....

Figure 4.2 The solid curves show the temperatures of onset of turbidity of solutions of Monoand Di-PDMS in hexane and diisopropylamine. The dashed curves show the temperatures of completion of gelation.

Figure 4.3 DSC curves for the Di-PDMS based gel, during heating, cooling and heating cycles, showing thermo-reversibility of gelation.

Figure 4.4 Optical micrographs of Di-PDMS gels with (a), (b), and (c) with propylamine; (d), (e), and (f) with hexane/water. (a) $3 \mathrm{mM}$ (b) $8 \mathrm{mM}$ (c) $8 \mathrm{mM}$ gel after one week. (d) 95/5 (e) 90/10 (f) $85 / 15(8 \mathrm{mM})$. The insets show the inverted vials containing the gels. .106

Figure 4.5 SEM images of (a) Di-PDMS/propylamine xerogel; (b) a cross-section of xerogel in (a); (c) Di-PDMS/hexane/water (8mM/90/10) xerogel; (d) Di-PDMS/hexane/water (8mM/85/15) xerogel

Figure 4.6 Transmission electron microscope images of Di-PDMS gels with (a) propylamine (b) hexane/water: 95/5 mixture.

Figure 4.7 (a) - (f): Thermo-reversibility of the gel by slow-cooling; (g) and (h): Irreversibility by quenching. .110

Figure 4.8 OM showing that Mono-PDMS gel is not reversible if the sample is quenched from the hot solution.

Figure 4.9 (a) Optical microscope image of Mono-PDMS/propylamine gel (b) SEM of the xerogel (c) SEM of fractured gel (d) TEM image of the xerogel. 
Figure 4.10 (a), (b) and (c): OM of gels with 80/20, 50/50 and 20/80 Mono- / Di-PDMS, respectively; (d), (e) and (f): SEM images of gels with 80/20, 50/50 and 20/80 Mono-/ DiPDMS, respectively

Figure 4.11 X-Ray diffraction traces from the xerogels of (a) Mono-PDMS and (b) Di-PDMS. The PDMS Mw $=1500$. .116

Figure 4.12 X-ray diffraction profile of films of (a) and (b) Mono-PDMS; (c) and (d): DiPDMS. (a), (c) with Mw of 900; (b), (d) Mw of 3000 for the PDMS segment.... 117

Figure 4.13 (a) UV-Vis absorption spectra of Mono-PDMS/propylamine gel with various concentrations; (b) Fluorescence spectra of Mono-PDMS gel with various concentrations; (c) UV-Vis absorption spectra of Di-PDMS/propylamine gel with various concentrations (d) Fluorescence spectra of Di-PDMS gel with various concentrations .119

Figure 4.14 Absorption spectra of xerogels of Mono- and Di-PDMS.....................120

Figure 4.15 Absorption spectra of gels and solutions of blends of Mono- and Di-PDMS......122 Figure 4.16 Absorption spectra of (a) Mono-PDMS gels in hexane/ water: 95/5 mixtures, with different concentrations. (b) Mono-PDMS gels (10mM) in hexane/water: 95/5 and 85/15. (c) DiPDMS gels in hexane/ water: 95/5 mixtures, with different concentrations. (d) Di-PDMS gels $(10 \mathrm{mM})$ in hexane/ water mixtures with different water concentrations.

Figure 4.17 Quantum Yields for Mono- and Di-PDMS Gels with propylamine and hexane/water mixtures. .126

Figure 4.18 Storage and loss moduli of the gels (a) Di-PDMS/propylamine (b) Di-PDMS/ (hexane/water: 95/5) (c) Mono-PDMS/propylamine (d) Mono-PDMS/ (hexane/water: 95/5)...127 


\section{CHAPTER 5}

Figure 5.1 (a) MJ-PTCDI with propylamine-water (99/1 v/v) (b) DJ-PTCDI with propylaminewater $(99 / 1 \mathrm{v} / \mathrm{v})$ (c) MJ-PTCDI with propylamine-water $(95 / 5 \mathrm{v} / \mathrm{v})$ (d) DJ-PTCDI with propylamine-water $(95 / 5 \mathrm{v} / \mathrm{v})(\mathrm{e}) \mathrm{MJ}-\mathrm{PTCDI}$ with cyclohexane-toluene $(95 / 5 \mathrm{v} / \mathrm{v})(\mathrm{f})$ DJ-PTCDI

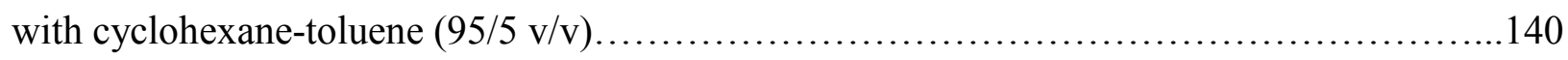

Figure 5.2 (a), (b) and (c): OM, SEM, and TEM, respectively of $10.00 \mathrm{mM}$ DJ-PTCDI gel with propylamine-water $(99 / 1 \mathrm{v} / \mathrm{v})$; (d), (e) and (f): OM, SEM and TEM, respectively, of $10.00 \mathrm{mM}$ MJ-PTCDI gel with propylamine-water. (g) SEM image of the cross section of DJ-PTCDI gel (h) cross section of MJ-PTCDI gel. 143

Figure 5.3 Two models of DJ-PTCDI packing; the substituents on both sides are hydrophilic. This would lead to a vesicular morphology. 144

Figure 5.4 SEM of Xerogel formed with cyclohexane/toluene $(95 / 5 \mathrm{v} / \mathrm{v})$ (a) MJ-PTCDI and (b) DJ-PTCDI; (c) MJ-PTCDI (d) DJ-PTCDI; Xerogel with cyclohexane/toluene (99/1 v/v) (e) DJ-PTCDI (f) cross section of DJ-PTCDI and (g) MJ-PTCDI. 145

Figure 5.5 DSC of $10.00 \mathrm{mM}$ gels with propylamine-water (99/1 v/v) (a) MJ-PTCDI (b) DJPTCDI .147

Figure 5.6 (a) UV-Vis absorption (b)Fluorescence of MJ-PTCDI gel with propylamine-water (99/1 v/v) (c) UV-Vis absorption (d) Fluorescence of DJ-PTCDI gel with propylamine-water $(99 / 1 \mathrm{v} / \mathrm{v})$

Figure 5.7 UV-Vis absorption (a) MJ-PTCDI gel (b) DJ-PTCDI gel with propylamine-water $(95 / 5 \mathrm{v} / \mathrm{v})$ .151 
Figure 5.8 (a) $10.00 \mathrm{mM}$ (b) UV-Vis absorption (c) Fluorescence spectra of MJ-PTCDI Gels; (d)10 mM (e) UV-Vis absorption (f) Fluorescence spectra of DJ-PTCDI Gels ; with

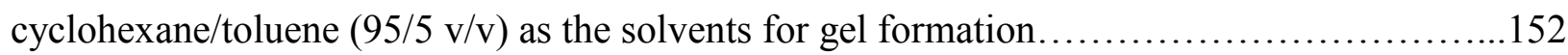

Figure 5.9 Variation of G' and G' with frequency (a) DJ-PTCDI and (b) MJ-PTCDI Gels with propylamine/water (99/1 v/v); (c) DJ-PTCDI and (d) MJ-PTCDI Gels with cyclohexane/toluene (95/5 v/v); (e) DJ-PTCDI (f) MJ-PTCDI Gels with cyclohexane/toluene (99/1v/v). .. .153

\section{CHAPTER 6}

Figure 6.1 Phase diagrams of gels formed with (a) cyclohexane (b) toluene (c) transdecalin 164

Figure 6.2 DSC traces of $8.00 \mathrm{mM}$ Gels in (a) cyclohexane (b) trans-decalin..................165

Figure 6.3 SEM of Xerogels from (a) Cyclohexane (b) Toluene (c) trans-decalin; and TEM of Xerogels from (d) Cyclohexane (e) Toluene (f) trans-decalin............................. 166

Figure 6.4 OM of Gels in trans decalin at: (a) 3.00 (b) 5.00 (c) 8.00 (d) $10.00 \mathrm{mM}$; (e) SEM and (f)TEM of Xerogel at $10.00 \mathrm{mM}$ .168

Figure 6.5 (a) UV-Vis of $10.00 \mathrm{mM}$ PSPerPS Gel from different solutions; (b) UV-Vis and

(c) Fluorescence Intensity of PSPerPS Gel from trans- decalin. 170

Figure 6.6 XRD traces of PS-PTCDI-PS Xerogels from (a) cyclohexane (b) trans-decalin.....172 Figure 6.7 Variation of G' and G' with frequency of PS-PTCDI-PS gels with (a) cyclohexane (b) toluene (c) trans-decalin. 174 


\section{CHAPTER 7}

Figure 7.1 OM images of PS/PS-PTCDI-PS films cast from different solvents at different concentrations.

Figure 7.2 SEM images of films at $5 \mathrm{wt} \%$ PS-PTCDI-PS from (a) chlorobenzene (b) chloroform and (c) THF

Figure 7.3 SEM of films at 10 wt \% PS-PTCDI-PS from (a) chlorobenzene (b) chloroform...183 Figure 7.4 Physical appearances of the PS/PS-PTCDI-PS films. $5 \mathrm{wt} \%$ PS-PTCDI-PS cast from (a) chlorobenzene (b) chloroform; $10 \mathrm{wt} \%$ PS-PTCDI-PS cast from (c) chlorobenzene (d) chloroform. 185

Figure 7.5 The variation of glass transition temperature is plotted as a function of PS-PTCDI-PS concentration in the PS polymer matrices using three different solvents

Figure 7.6 XRD patterns of three PS/ PS-PTCDI-PS (5 wt \%) films drop cast from different solvents.

Figure 7.7 UV-Vis absorption spectra of films drop cast from different solvents at different PS-PTCDI-PS concentrations.

Figure 7.8 IR spectrum of pure PS film cast from chloroform (top) compared to that of the PS/PS-PTCDI-PS (5 wt \%) blended film cast from chloroform (bottom).

Figure 7.9 IR spectrum of PS/PS-PTCDI-PS (5 wt \%) blended film cast from THF. 


\section{List of Appendices}

Appendix

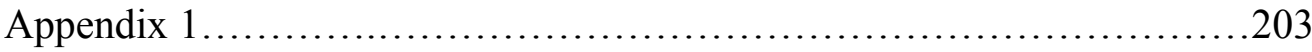

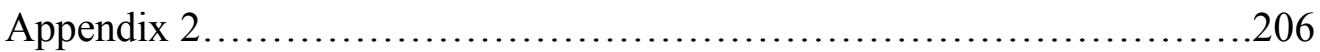

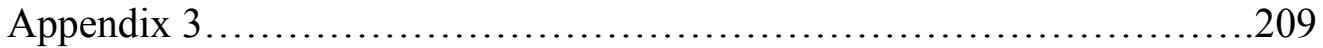

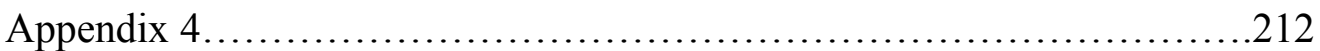

\section{List of Abbreviations}

PDMS Polydimethyl siloxane

PTCDI Perylene-3,4,9,10-tetracarboxylic diimide

MJ-PTCDI Mono Jeffamine monoisopropyl perylene tetracarboxylicacid diimide

DJ-PTCDI Jeffamine perylene tetracarboxylicacid diimide

FT-IR Fourier Transform Infrared Spectroscopy

NMR Nuclear Magnetic Resonance Spectroscopy

$\AA \quad$ angstroms or $1 \times 10^{-10}$ meters

PS Polystyrene

PS-PTCDI-PS Polystyrene perylene tetracarboxylicacid diimide

$\mathrm{M}_{\mathrm{w}} \quad$ Weight average molecular weight

OM Optical Microscopy

SEM Scanning Electron Microscopy

$\mu \mathrm{m} \quad$ micrometer

$\mathrm{nm} \quad$ nanometers

DSC Differential Scanning Calorimetry 


\title{
CHAPTER 1
}

\author{
Introduction
}




\subsection{Basic Principles of Self-Assembly}

Molecular self-assembly is the spontaneous association of molecules under equilibrium conditions into stable, structurally well-defined aggregates that are joined mainly by noncovalent interactions such as hydrogen bonding, $\pi-\pi$, hydrophobic and ionic interactions. The key factors in self-assembly are structural compatibility and complementarity. The morphologies and thermal behavior of the compounds are important factors in designing functional materials, and these properties change depending on the interactions mentioned above. An example of selfassembly is provided by the protein-enzyme lock-and-key fit in nucleic acids, ${ }^{1}$ which, by virtue of its simplicity, has inspired the design of non-biological, self-assembling structures. Familiar examples are the formation of double-stranded DNA by association of two complementary chains of DNA, ${ }^{2}$ and the intra-molecular folding of t-RNA. ${ }^{3}$ These structures rely, in part, on the complementary patterns of donation and acceptance of hydrogen bonds. These patterns can be replicated synthetically, and hydrogen bonds are substantially better defined in their directionality than are van der Waals interactions. Thus, molecules that are capable of forming networks of hydrogen bonds have become the foundation for the majority of the current research works in molecular recognition and self-assembly.

The field of supramolecular chemistry has grown exponentially in the last few decades, as indicated by the large number of articles, reviews, and books that address this subject. ${ }^{4 a-c}$ The recent development of "bottom up" methods to create nanostructures was inspired primarily by Nature, where there is a wide variety of complex nanostructures with astonishing precision. ${ }^{5,6}$ Precision and specificity are indicative of the control and directionality that result from the secondary interactions between complementary components in biological systems. However, the challenge is to create structures synthetically with similar precision and specificity by cleverly 
incorporating complementary recognition sites in the molecular components for secondary interactions. This challenge can be met only if we can first understand how molecular selfassembly in biological systems operates to generate well-defined aggregates. Then, the knowledge that is acquired can be transferred from biological systems to synthetic systems. Thus, understanding the supramolecular chemistry can have a profound effect on how efficiently chemists can use spontaneous secondary interactions such as hydrogen bonding, dipole-dipole interactions, charge transfer, van der Waals interactions and $\pi$ - $\pi$ stacking interactions, ${ }^{5}$ to prepare structures of different sizes and shapes with dimensions in the range of 1 to $100 \mathrm{~nm}$, as well as micron-scale self-assembly systems including the materials used in the research for this thesis.

The scientific community continues to use natural systems as an archetype, since all organisms in Nature use self-assembly to facilitate rapid expansion from small units into higherordered supramolecular structures. The fidelity of these self-assembled structures is regulated by the complementarity of their recognition units, and the final supramolecular assemblies are often multifunctional, i.e., they are able to perform a variety of tasks. Over the past three decades, polymer chemists have been striving to understand the basic principles associated with the hierarchical formation of the structure of biopolymers, and their work has resulted in significant progress in the field of synthetic polymer chemistry. However, at present, there are very few, if any, tools available that can exert a level of control of polymer structures that is comparable to that of biological systems. ${ }^{4 b}$ To date, the most successful attempts to prepare hierarchicallystructured polymer materials were based on combined strategies that made use of the achievements of several research areas, such as modern organic and polymer chemistry, ${ }^{5}$ materials science, and supramolecular chemistry. ${ }^{5,6}$ Thus, the supramolecular self-assembly of monodisperse, $\pi$-conjugated oligomers has been studied extensively as a means of creating 
hierarchically-structured, opto-electronically-active materials. An example in the recent advances in the field of optoelectronic devices ${ }^{6}$ is aimed at the development of new generations of biomedical devices. Most opto-electronic materials require conjugated systems, and conjugated molecules are known to aggregate due to the so-called " $\pi$ - $\pi$ interactions," a term used to describe the coupling between the $\pi$ electron clouds of adjacent aromatic molecules. The solid-state packing that results from the intermolecular interactions of these molecules impacts charge transport, ${ }^{6}$ thus it has a great influence on the opto-electronic properties and the performance of devices made of such compounds. Future advances in this field will require the development of synthetic materials that offer diverse chemical functionality and a degree of hierarchical structural order similar to that of biomaterials.

\subsection{Perylene Diimides: Building Blocks for Functional Supramolecular Architectures}

Due to the intrinsic insolubility of perylene diimides (PDI), their potential as fluorescent dyes with high fluorescence quantum yield and photostability was not discovered until 1959. ${ }^{14}$ Since that time, however, they have become the basis for extensive research on light-induced energy and electron transfer processes, ${ }^{15-17}$ and more application-focused studies have been conducted in the field of laser dyes and fluorescent light collectors. ${ }^{18-21}$ In recent years, significant effort has been devoted to the study of the self-assembly of $\pi$-conjugated organic materials, because proper ordering of the functional molecular building blocks is essential for the development of electronic and photonic organic materials that have competitive performance to their inorganic counterparts. For example, PDIs are the best $n$-type semiconductors available to date, and the most recent applications of PDI pigments are in the field of electronic materials. ${ }^{7,8}$ This n-type semiconductivity is related to the high electron affinity of perylene diimide dyes, ${ }^{9}$ 
which makes naphthalene, perylene, and higher rylene diimide dyes ${ }^{10}$ most promising for application in organic field-effect transistors. ${ }^{11}$ In addition, based on their unique combination of optical, redox, and stability properties, PDI dyes have been investigated for a few decades in the fields of electrophotography (xerographic photoreceptors) ${ }^{12}$ and photovoltaics. ${ }^{13}$ Over the past few years, there has been increasing interest in this class of chromophores because of their favorable properties mentioned above. Thus, perylene monoimide and diimide dyes have proved to be the best fluorophores currently available for single molecule spectroscopy. ${ }^{22}$

Since the discovery of the intense yellow-green photoluminescence of the parent perylene diimide1 (PDI1) (Scheme 1), numerous PDI dyes have been synthesized for use as fluorescent standards, in fluorescent light collectors, or as laser dyes. ${ }^{23}$ For such applications, it is essential for the imide substituent to have a negligible influence on the absorption and emission properties of PDIs because of the nodes of the highest occupied molecular orbital (HOMO) and lowest unoccupied molecular orbital(LUMO) at the imide nitrogens. Therefore, PDIs can be regarded as closed chromophoric systems with an $\mathrm{S}_{0}-\mathrm{S}_{1}$ transition (polarized along the extended molecular axis) in which the intensity and position remain unaltered by the respective imide substituents. ${ }^{43}$ Accordingly, imide substituents are well suited for tailoring application-directed properties such as solubility, and for preventing aggregation, which has a pronounced influence on the optical spectra. 

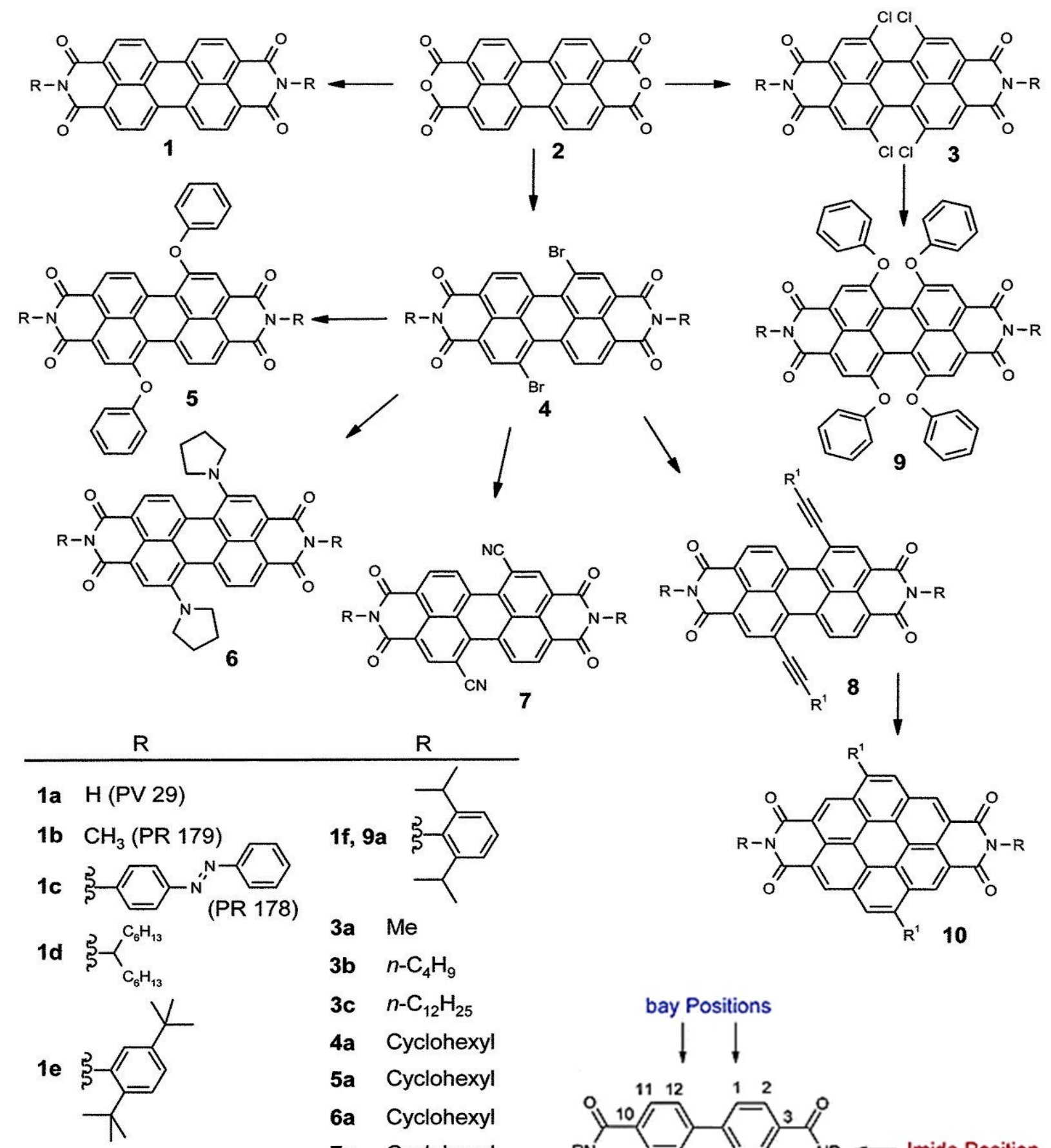

\section{$\mathrm{R}$}

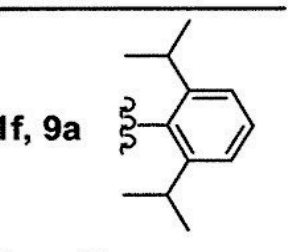

3a $\mathrm{Me}$

3b $\quad n-\mathrm{C}_{4} \mathrm{H}_{9}$

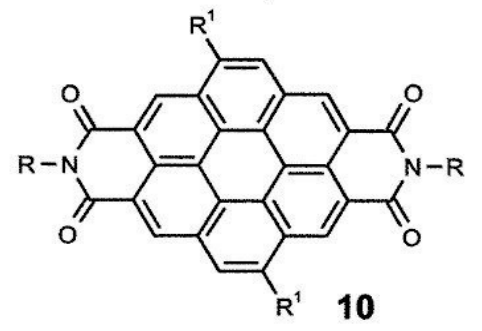

3c $n-\mathrm{C}_{12} \mathrm{H}_{25}$

4a Cyclohexyl

5a Cyclohexyl

6a Cyclohexyl

7a Cyclohexyl

8a Cyclohexyl $\mathrm{R}^{1}=n-\mathrm{C}_{10} \mathrm{H}_{21}$

10a Cyclohexyl $\mathrm{R}^{1}=n-\mathrm{C}_{10} \mathrm{H}_{21}$

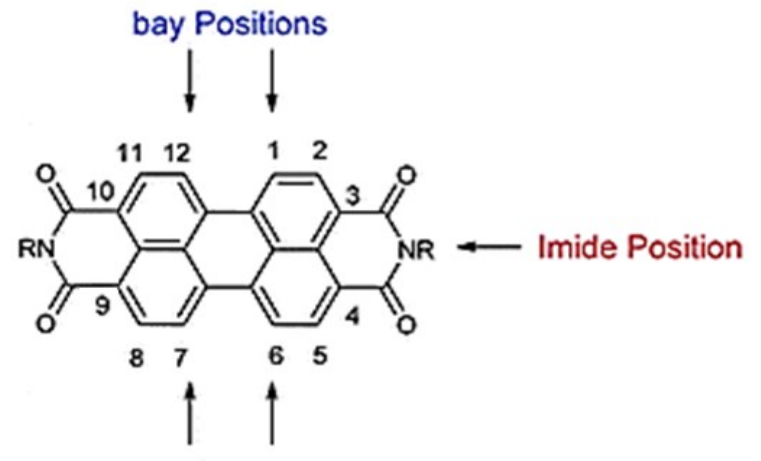

bay Positions

Scheme 1.1 Perylene diimide dyes that have been studied for their optical properties. ${ }^{45}$ 


\subsubsection{The Optical Properties of Perylene Diimides in Solution}

When dispersed in the common organic solvents, PDIs have large extinction coefficients at visible wavelengths (400 - $600 \mathrm{~nm}$ ); fluorescence quantum yields of almost unity; and long, singlet-excited-state lifetimes $(\sim 4 \mathrm{~ns}) .^{30-32}$ Generally, PDIs are characterized by a vibronicallystructured band with strong absorption in the visible region between 400 and $550 \mathrm{~nm}$, and they exhibit a strong yellow-green fluorescence as a mirror image of the absorption in common organic solvents. ${ }^{29}$ It has been reported that the electronic transitions for unsubstituted PDIs are predominantly HOMO to LUMO transitions. ${ }^{29}$ The absorbance of the $(0,0)$ vibronic transition at $\sim 527 \mathrm{~nm}$ and the $(0,1)$ vibronic band at $\sim 490 \mathrm{~nm}$ for free PDI molecules in solution shows the relationship to be $(0,0) /(0,1)>1.6$, and molecular aggregation of PDI dyes causes a significant decrease in this value. ${ }^{29,31}$

Similar phenomena also have been observed in the emission spectra of PDI in solution. As expected, substituents on the aromatic core bay-positions (i.e., positions 1, 6, 7, and 12 in Scheme 1) have much more obvious effect on the absorption and emission spectra of PDIs than substituents on the N-terminal groups due to the stronger electronic coupling between the PDIs' $\pi$-orbital and the substituents on the aromatic bay region. For example, two phenoxy substituents on the PDI bay positions result in $\sim 20 \mathrm{~nm}$ and $40 \mathrm{~nm}$ bathochromic shifts in the absorption and emission maxima ( $\lambda_{\mathrm{abs}}$ and $\lambda_{\mathrm{em}}$ ), respectively, than those associated with unsubstituted PDI dyes, and the color of the fluorescence changes to orange. ${ }^{29,32}$ More pronounced spectral changes occur upon substitution of the aromatic cores with electron donor groups, such as pyrrolidino, which gives PDI derivatives a dark-green color, both in the solid state and in solution, due to the bathochromic shift that exceeds $150 \mathrm{~nm}$. In contrast, limited changes in the fluorescence 
quantum yields were observed for PDI with electron-withdrawing substituents on the bay positions (dyes $3,4,7) .^{29,32}$

Langhals's swallow-tail-substituents ${ }^{24}$ have very high solubility (as in derivative 1d). For the longer homologue with 1-nonyldecyl chains, a solubility of as much as $35 \mathrm{~g}$ in $100 \mathrm{ml}$ of $\mathrm{n}$ heptane has been reported. ${ }^{24}$ For most applications (such as coloration of plastics), twisted aryl groups such as those that occur in $\mathbf{1 e}$ and $\mathbf{1 f}$, seem to be more advantageous because they increase solubility and prevent aggregation by steric means ${ }^{20}$ (Scheme 1). With two phenoxy groups attached at positions 1 and 7, the absorption maximum shifts by about $20 \mathrm{~nm}$, and with four phenoxy groups the maximum shifts by almost $50 \mathrm{~nm}$ compared to that of the unsubstituted dyes $\mathbf{1}$. The color of the fluorescence changes to orange (dye 5) and red (dye 9). In both cases, all favorable properties of these perylene fluorophores are maintained, e.g., high fluorescence quantum yield, small effects of the solvent on the optical properties, and high photostability. ${ }^{25}$ The dyes ${ }^{10}$ have a green color due to the two electron-donating pyrrolidino groups that produce a bathochromic shift of $160 \mathrm{~nm}$, resulting in emission in the infrared region. Since this spectral shift is caused by charge transfer, pronounced solvatochromism is observed for these green dyes, and their fluorescence quantum yield is decreased. In the case of coronene bisimide 10, significant changes in the absorption spectra occur that are not typical for PDI dyes. ${ }^{25}$ Accordingly, new electronic properties arise by expansion of the p-conjugated system orthogonal to the imide-imide axis.

From the structural point of view, a reasonable approach for obtaining supramolecular PDI dye assemblies is the modification of these chromophores in such a way that structural growth (or 'self-assembly') becomes possible only in one or two dimensions. Intermolecular interaction in a third dimension, which could lead to the formation of crystalline solids, is 
prevented by attaching appropriate substituents to the molecules. An example of such an approach is Seybold ${ }^{20}$ dye 9a, in which the bulky phenoxy groups strongly interfere with the aggregation of the p-systems, resulting in remarkably high solubility. In this dye, the imide substituents are positioned properly for the introduction of supramolecular receptor units. ${ }^{20}$ Since the most promising receptor units in supramolecular chemistry are particularly those that enable non-covalent interactions such as hydrogen-bonding and $\pi$ - $\pi$ stacking, the ultimate aim is to design a polymer with receptor units that direct the self-assembly of the individual building blocks in a defined manner.

\subsubsection{Crystalline State of Perylene Diimides}

The $\pi$-interaction mediated self-assembly of the perylene segment is influenced by the substituents. Some of the short linear substituents lead to liquid crystalline behavior; in fact, most of the research on perylene diimides has been done with short side-group substitutions at the imide position since it has been shown that attachment of longer chains to PDIs impedes liquid crystalline ordering (Figure 1.1). According to X-ray diffraction studies of single crystals, ${ }^{26,27}$ PDIs are flat, planar $\pi$-systems; in the solid state, these platelet-like molecules form stacks. The packing of PDIs has been studied in detail by Graser, Hädicke, and Klebe. ${ }^{26}$ In their papers, they presented the crystal structures of 18 PDIs that they had determined in their research. The only difference between these 18 diimides is the imide substituent. Six more derivatives were reported recently by Zugenmaier et al. ${ }^{27}$ All of these imides exhibit similar crystal structures, i.e., planes of PDIs arranged in parallel stacks that are about 3.3 to $3.6 \AA$ apart. ${ }^{26}$ However, the perylene planes do not fully overlap with each other. The offset differentiates one perylene from another. Graser et al ${ }^{26}$ set up a model that defined a transverse offset, which is the cross-shift of an imide, and a longitudinal offset, which is the shift along the imide (Figure 1.1). The color of a dye is 
determined by the electronic properties of the individual molecular chromophores and by the electronic interactions with the vicinal molecules in the crystal. In the PDI packing structure, the interaction between two planes is via $\pi-\pi$ stacking.

(a)

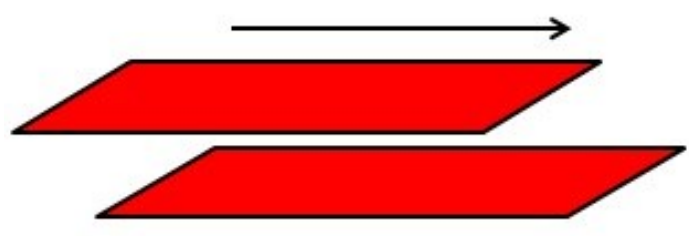

(b)

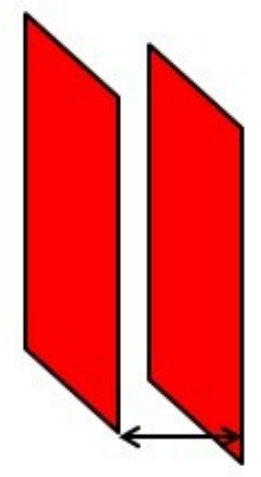

(d)

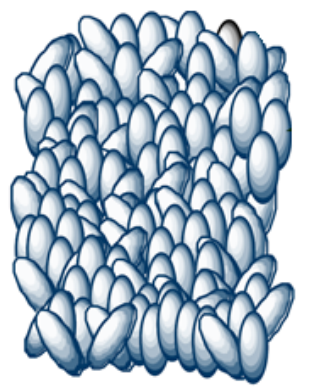

LC phase

\section{Crystalline \\ solid}

Figure 1.1 $\pi-\pi$ stacking of perylene diimides involving (a) longitudinal and (b) transverse offsets (c) crystalline solid packing (d) LC phase

Different substituents change the color of diimides due to the difference between the transverse and longitudinal offsets. Thus, PDIs may exhibit very different colors in the solid state depending on the packing of these diimides. According to Graser et al.'s theory, ${ }^{26}$ the color of the band in the absorption spectra depends on the projected overlap of neighboring perylene skeletons. An empirical model was developed for the absorption maxima of the 18 PDIs in the solid state as functions of three crystal parameters, i.e., perylene-perylene stacking distance (d), 
transverse offset shift (t), and longitudinal offset shift (1). However, 'd' was not included in the empirical equation because most PDIs have similar interplanar d-spacing of about $3.5 \AA$ in their crystal structures. As a result, the following equation gives the best determination of the maximum absorption $\left(\lambda_{\max }\right)$ :

$$
\lambda_{\max }=9.718 t^{2}-82.009 t-21.888 l+735.329
$$

The longitudinal shift causes a linear change in the wavelength, while the change in the maximum absorption has a much more significant dependence on the transverse shift. Therefore, the differences in the colors of the diimides are affected to greater extent by the transverse shift than by the longitudinal shift. Since the width of the PDI skeleton is about $4 \AA$, the transverse shift cannot exceed this distance. Otherwise, there is no overlap between neighboring molecules. Theoretically, the maximum of absorption will be $735 \mathrm{~nm}$, if the two molecules are superimposed. However, to avoid unfavorable interactions between the packed molecules in all compounds, a relatively longitudinal and transverse shift was observed. For the $24 \mathrm{PDIs},{ }^{26,27}$ the longest wavelength found was $625 \mathrm{~nm}$.

The side chain of the PDI has a crucial role in controlling the molecular packing conformation. ${ }^{26,27}$ For example, changing the side chain from ethoxyethyl to methoxypropyl transforms the color of the perylene aggregate from red to black. ${ }^{26 e}$ Obviously, the observed spectral change in these solids is the manifestation of an aggregation effect, because the molecules have the same absorption spectra in solution. Hence, side-chain modulation provides a potentially effective way to control and optimize the molecular packing conformation, thus enabling the 1D growth of the self-assembly. Also, the two nitrogen positions at the imides of PDI are nodes in the $\pi$-orbital wave function, ${ }^{28}$ and they provide countless options for modifying the structures of the two side chains without significant alteration of the electronic properties of 
the perylene molecule. This makes it feasible to examine the effect of the side chain on the molecular packing conformation, hence the morphology of the molecular self-assembly.

\subsection{Organogels vs. Polymer Gels}

Physical gels, a form of soft matter, have been exploited for many practical purposes for several centuries. The hierarchical formation of 3D network superstructures from self-assembled nanostructures that are reversible and controllable may be useful for various applications ${ }^{33}$ in the design and processing of innovative devices. In the past, many studies have been conducted concerning structurally diverse gelator systems, e.g., systems based on saccharides, ${ }^{34}$ peptides and ureas, ${ }^{35}$ nucleobases, ${ }^{36}$ steroid derivatives, ${ }^{37}$ and dendrimers. ${ }^{38}$ Recently, in the field of photofunctional gels, "designed" dye molecules have been introduced in various applications such as sensors, optoelectronic devices, and light-harvesting modules. ${ }^{4 c}$ However, despite the growing number of studies on structurally-diverse gelators and their technological applications, it is surprising that a fundamental understanding of the specific characteristics of molecular selfassembly and gelation is still quite limited. ${ }^{34}$ The difficulties associated with understanding the mechanism of gelation arise from the fact that significant research efforts are still being devoted for identifying the potential molecular architectures of gelators by screening large numbers of compounds and testing a wide range of solvents that may be suitable for supporting gel-phase networks. Hence, an understanding of the structure-activity relationships that support and promote self-assembly into gels would enhance the probability that systems could be designed in which molecular modification could control nanoscale assembly, and ultimately endow specific functionalities to various molecules. 
Gelation can particularly occur with two different systems: from organic molecules that self-assemble into organogels; or from polymer solutions, which once cooled to below the gelation threshold, produce physical gels. ${ }^{57}$ Polymer gels and organogels are made up of different building blocks, but they are very similar as far as morphology and rheological properties are concerned. Both systems are thermo-reversible which means they can be melted and reformed at will through heating and cooling cycles without altering the chemical structures of their constituents. Organogelators self-assemble via one-dimensional growth modes to form fibers, strands, or tapes that are frequently crystalline. The primary nanoscale objects, regardless of their shapes, join in three-dimensional networks that encapsulate the liquid component, and inhibit its flow. However, it is generally assumed that strong intermolecular forces, such as $\mathrm{H}-$ bonding, electrostatic attractions, and $\pi-\pi$ stacking interactions are necessary to stabilize these assemblies. Conversely, polymer gels are regarded as three-dimensional networks of macromolecules that are physically or chemically cross-linked (the physical cross-link comes about by chain entanglement as shown in Figure 1.2 (c)). This polymer network consists of several components including bridging strands, cross-links (or junctions), dangling ends, and loops that trap the solvent. Organogels and polymer gels are compared below to highlight their differences and similarities. 


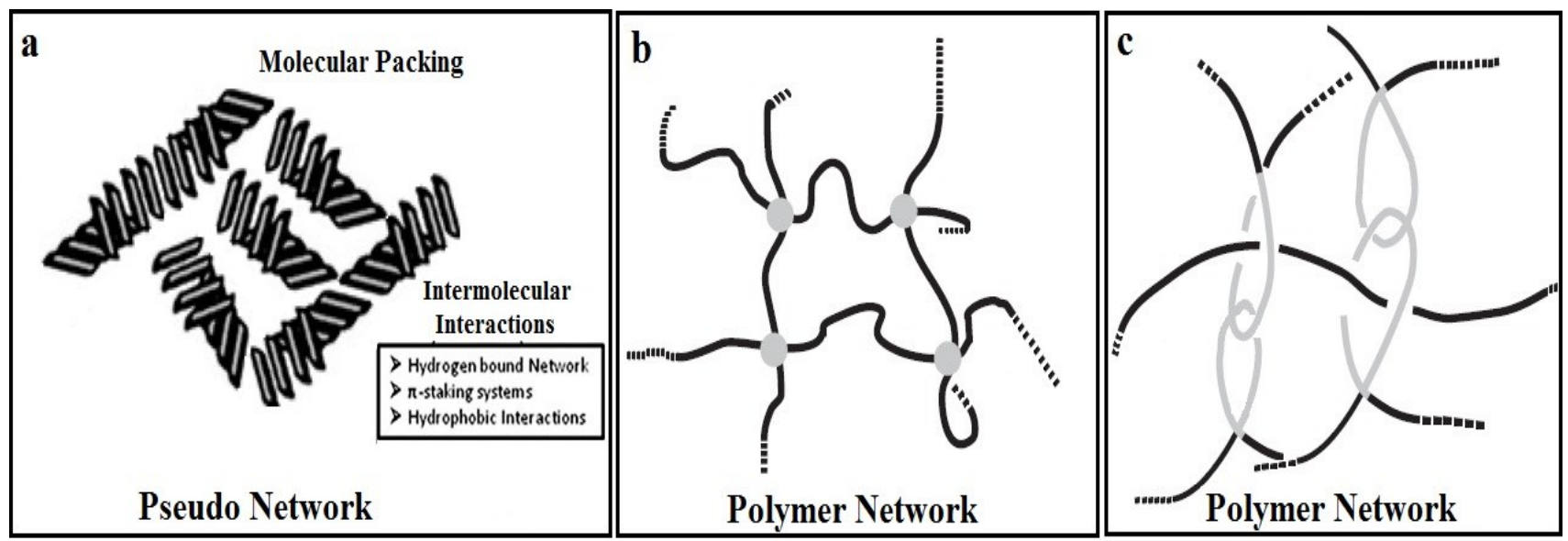

Figure 1.2 Schematic representation of a) the pseudo network of LMOG; b) a chemically crosslinked network of polymers; c) entangled physical network of polymers

Organogels are thermo-reversible, quasi-solid, soft materials that consist of lowmolecular weight organogelators (LMOGs) self-assembled in organic liquids (usually $\geq 95 \mathrm{wt} \%$ ). LMOGs self-assemble via physical interactions including hydrogen-bonding, ${ }^{39-42} \pi-\pi$ stacking, ${ }^{43}$ dipole-dipole interactions, ${ }^{44}$ and London dispersion forces, ${ }^{45}$ into 3 -dimensional networks. ${ }^{46-48}$ The solvent-gelator specific intermolecular interaction (i.e., H-bonding) and non-specific intermolecular interactions (dipole-dipole, dipole-induced, and instantaneous dipole-induced forces) are equally important in establishing a continuous three-dimensional network. ${ }^{49,}{ }^{50}$ In molecular gels, self-assembly is an intricate process that must balance solubility and the intermolecular forces that control growth into axially-symmetric, elongated aggregates. ${ }^{47,} 50-53$ During assembly, individual small molecules are driven to aggregate into oligomers by molecular self-recognition and intermolecular non-covalent interactions, and, subsequently, these oligomers assemble into fibrillar aggregates that immobilize the solvent via capillary forces. ${ }^{54,55}$

Physical gelation in polymers is considered to be the result of liquid-liquid phase separation and vitrification caused by the spinodal decomposition mechanism. ${ }^{56,57}$ Such a 
phenomenon could also be accompanied by crystallization in the case of semi-crystalline polymers. ${ }^{58}$ Gels with crystallizable polymers such as isotactic polystyrene, poly (vinyl chloride), and polyethylene have been studied, ${ }^{59,60}$ and liquid-liquid phase separation accompanied by crystallization has been attributed to the formation of networks. The gel of atactic polystyrene (aPS), ${ }^{61}$ which is a non-crystallizable polymer, has also been found to form networks or spherical domains consisting of networks with cyclohexanol as the solvent. The thermo-reversible gelation of a-PS is of considerable interest because the mechanisms of physical cross-linking, which generally are thought to be involved in reversible network formation, seemingly do not apply. Comparison of the gelation behavior of a-PS with that of crystalline polymers revealed several important similarities that suggested that the phenomena are closely related. Gelation of a-PS showed an inverse square dependence of the critical gelation concentration on the molecular weight, indicating that chain overlap is a necessary condition for gel formation. In order to probe further the relationship between gels of crystalline and non-crystalline polymers, the thermo-reversible gelation of a series of chlorinated polyethylenes was investigated previously, ${ }^{62}$ and it was concluded that while crystallization may contribute to the nonequilibrium characteristics of thermo-reversible gelation, gelation of the non-crystalline, chlorinated polyethylenes demonstrates that crystallinity is not required for gel formation. Atactic polystyrene also undergoes thermo-reversible gelation, ${ }^{61}$ and as in the case for noncrystalline chlorinated polymers, the gel formation and gel melting temperatures of a-PS are identical. ${ }^{62}$ This suggests that the mechanism of physical cross-linking that produces the gel network in non-crystalline polymers is an equilibrium process that does not involve crystallization in the conventional sense. 


\subsubsection{The Principles of Gelation}

The first attempt to link macroscopic and microscopic properties to define a gel was made by Hermans, ${ }^{63}$ and it served as the basis for Flory's much more comprehensive definition. Flory defined a gel as a two-component, colloidal dispersion with a continuous structure with macroscopic dimensions that is permanent on the time scale of the experiment and has a rheological behavior akin to that of a solid. ${ }^{63}$ Based on this definition, any gel must develop a three-dimensional network that permeates its volume and remains stable within specific ranges

of concentration and temperature. In a Faraday Discussion meeting on Gels in 1995, Keller ${ }^{64}$ discussed various structures in more detail. He illustrated gel morphology schematically as the liquid-liquid phase separation intercepted by vitrification. Keller defined a "polymer gel" as follows:

(1) A polymer-rich phase connected by isolated, solvated chains

(2) Continuously connected phases

(3) Glassy solid phase containing solvent-rich phases

(4) Glassy dispersed phase connected through adhesive contact

Keller also identified two classes of gels that arise from the liquid to crystalline phase transformation, as follows:

(1) Junctions, bundles, micelles, or aggregated rigid chains connected by released chains

(2) Network elements formed by fibrous crystals constituted by rigid or semi-flexible chains that do not fold on crystallization

Today, gels are classified based on the Nature of the bond involved in the cross-linking region, and the classification of gels as chemical gels (irreversible) and physical gels (reversible) is widely accepted. In the chemical gels, the cross-linking region is formed by covalent bonds, 
while in the physical gels, that region is formed by hydrogen bonds, ionic bonds, and hydrophobic interactions. Therefore, self-complementary, unidirectional, intermolecular interactions can be considered as prerequisites for organo-gelation. ${ }^{65}$ Although a wide range of gelators has been reported, it is unfortunate that, so far, the development of new gelators is more a serendipity than a planned event. ${ }^{66}$ Thus, the question arises concerning how to design a system that can gel one or more specified organic solvents. As noted by Weiss ${ }^{67}$ and coworkers, the following aspects should be taken into consideration:

(1) The structural requirements for a molecule to gel the solvent

(2) The relationship (if any) between the packing arrangements of gelator molecules in their bulk crystalline states and in their various aggregates in the gels

(3) How the molecular packing of gelators in their aggregate states influences the mechanical, thermodynamic, optical, and other properties of the gels

The morphology of the self-assembled gels may be either discrete constructions, such as empty vesicles or extended assemblies, such as fibrillar networks. These assemblies in turn trap the solvent in a 3D network. Many groups have tried to elucidate the correlations among structures and electronic properties of the gelator and the solvent, as well as the physical properties of their gels. The commonality for designing a gelator based on the results reported before is that the formation of gels indicates a hierarchical process ranging from individual dissolved molecules to $3 \mathrm{D}$ entangled superstructures. ${ }^{68}$ Therefore, by rational design of molecular structures and precise control of their aggregation by altering various factors, such as the solvent, temperature, and concentration, it is possible to direct the self-assembly pathway towards the formation of gels. 


\subsubsection{Reversibility of Gels}

Most supramolecular gels are characterized by a gel to solution (gel-to-sol) transition at moderate temperatures, since the strength of non-covalent bonding is comparable to ambient thermal energy. ${ }^{69}$ Thus, supramolecular gels lack robustness even though these are very adaptive in regard to their responsiveness to stimuli. In general, it is a significant challenge to design of an extensive supramolecular three-dimensional network ${ }^{65}$ that is both robust $^{69}$ and adaptive. Usually, gels are prepared by warming a small amount of gelator (0.1-10 wt\%) in an organic solvent (or water for hydrogels); upon cooling below the temperature of gelation ( $\left.\mathrm{T}_{\text {gel }}\right)$, the entire solvent is immobilized and can support its own weight without collapsing. This is frequently tested by turning the test tube upside down; if no flow is observed, the solution is said to have gelled (Figure 1.3). Hence, the self-assembled aggregates in the gel are linked in complex, threedimensional networks that immobilize the liquid component to variable extents. ${ }^{65}$

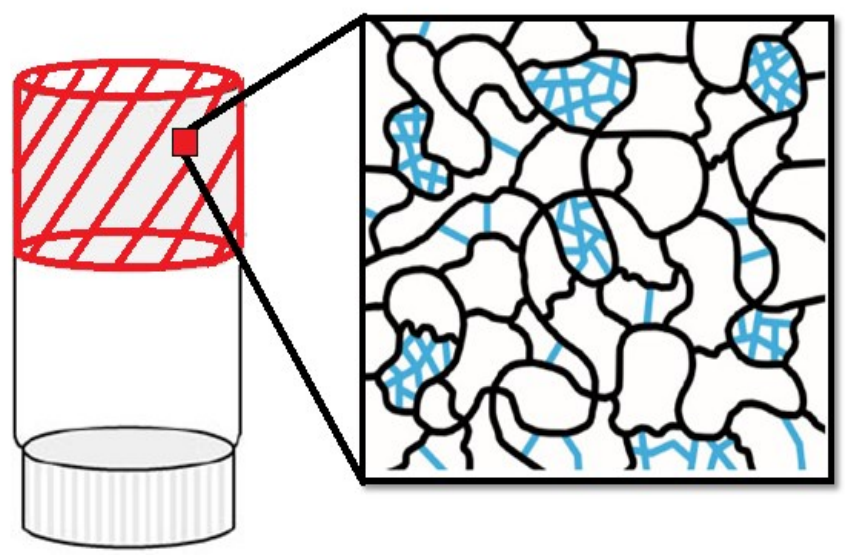

Figure 1.3 Formation of a gel is tested by "stable-to-inversion" of a vial

The 3D superstructures are maintained by a subtle balance between gelator-gelator interaction and gelator-solvent interaction. In addition to the self-driven factors that influence the 
formation of a gel such as temperature and concentration, external driving forces have a significant effect on the reversibility of the gels. A gelator's gelation ability in a specified solvent can be evaluated by critical gelation concentration (CGC), which indicates the lowest concentration of gelator molecules required for gelating a liquid at room temperature. At a certain concentration, the gel becomes thermo-reversible, and the phase transition temperature ( $T_{\text {gel }}$ from solution to gel (sol-to-gel) can be determined. The methods used for this purpose include differential scanning calorimetry (DSC), temperature-sweep rheological experiments, and hot-stage microscopy.

\subsection{Organogels Based on Perylene Diimide Dyes}

Many efforts have been made to incorporate functional dye molecules into gelators, and there have been extensive studies on the gelation of self-assembled mono- and di-substituted PDIs mediated by $\pi$ interaction. ${ }^{74,75}$ Many researchers, including Shinakai, Würthner, and Yagai, have reported the development of PDI-based organogelators. ${ }^{70-73}$ In these studies, well-defined, fibrous aggregates were observed by atomic force microscopy (AFM), and their formation has been attributed to $\pi-\pi$ stacking and intermolecular hydrogen bonding between the PDI molecules (Figure 1.4).

Achieving supramolecular control over these chromophore-linked molecular systems continues to be a challenge. The main challenge of 1D self-assembly of PDI molecules lies in controlling and optimizing the strong $\pi-\pi$ interaction between the perylene planes and the intermolecular interactions between the side chains. ${ }^{76}$ The self-assembly of the gelator depends upon a variety of factors including the solvent, concentration, temperature, and, above all, the molecular structure. A minimal structural alteration such as the length of the flexible side chain of a gelator is often crucial for the manipulation of the gelation-induced properties depending on 
the subtle hydrophobic/ hydrophilic balance. For example, most gelators have been reported to form a gel in a single solvent. However, a mixture of solvents could enhance the supramolecular organization of the gelator. Therefore, an optimum balance between the hydrophilic and hydrophobic groups in the gelator, obtained either by varying the molecular structure or changing the polarity of a solvent mixture, could control the gelation-assisted properties. 

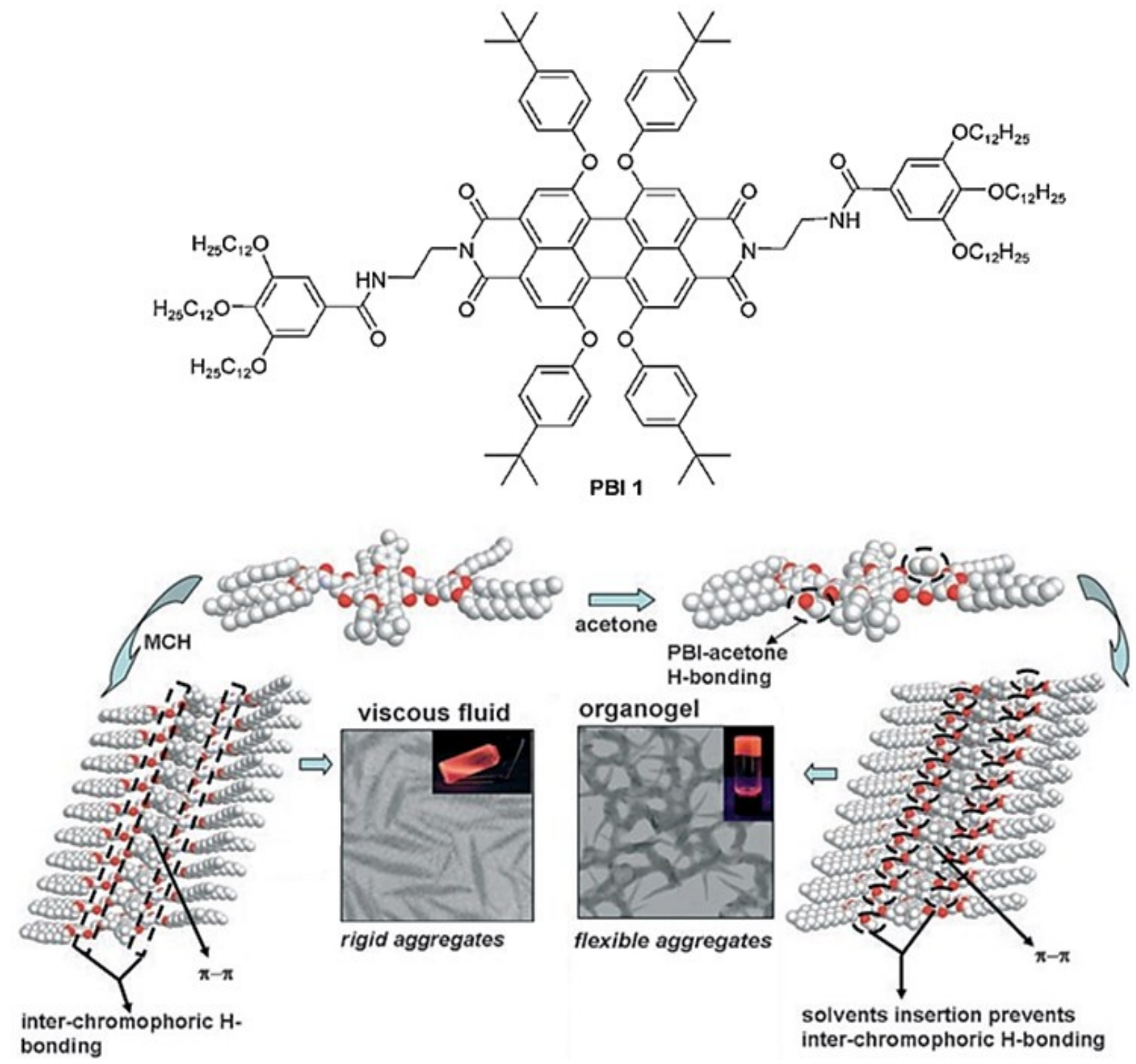

Figure 1.4 Schematic illustration of the formation of viscous lyotropic fluids and gels from

PBI 1 in methylene chloride and acetone $\mathrm{e}^{73}$ 


\subsection{Aim of this thesis work}

Most of the research on PDIs has been done with short side-group substitutions at the imide position. ${ }^{77}$ It has been shown that attachment of longer chains to PDIs impedes liquid crystalline ordering. ${ }^{78}$ Controlled aggregation of photoactive pigments in polymer matrices via $\pi$ interaction-mediated interaction has potential applications in solar cells. Our group previously studied the self-assembly of polymer-attached perylene diimides in aqueous and non-aqueous solvents and in films. The aim of my thesis is to study the gelation of covalently attached PDI polymers during their self-assembly. Polymer gelation is a route to obtain highly-organized morphologies. The formation of organogels involves small molecule gelators, which is distinctly different from formation of polymer-based gels. In organogels, network formation and solvent trapping depend on self-assembly of the small molecule gelator via non-covalent interactions such as hydrogen bonding. In contrast, in the polymer gelators, chain entanglement and network formation trap the solvent causing gelation, as observed in case of a-PS. Forces such as hydrogen bonding are not a requirement.

In this thesis, the results of the study of five polymer-based gel systems will be presented in five different chapters. Following is an overview of the chapters:

Chapters-1 and -2: These are introduction chapters to the work presented throughout this thesis as well as the materials and methods used to carry out the projects.

Chapter-3: The preparation of gels of poly (dimethyl siloxane) (PDMS) reported in the literature to date involves catalysts and chemical cross-linkers (chemical gels) or functionalization with organogelators. In this chapter, we report thermo-reversible, physical gels of PDMS without cross-linking or functionalization. PDMS is most commonly used as a sealant or separation layer for volatile organic components. It possesses properties such as high 
hydrophobicity, permeability, and good thermal and mechanical resistance, which make it an interesting membrane material for organic extraction and is easy to manufacture. Membrane transport properties depend on the micro-structure of the polymer network, that means those properties are influenced by the preparation conditions of the polymer. Hence, this made us to undertake the study of the gelation ability of PDMS first before exploring the PDMS substitutedPDIs and the other side-group substituents.

Chapter-4: A rod-coil system consisting of polydimethylsiloxane and perylene diimide (PDMS-PDI), previously studied by our group, showed behavior similar to that of amphiphilic block copolymer systems with micellar or vesicular morphologies depending on the blockspecific solvent used. The PDMS-PTCDI behaved like an inverse macromolecular surfactant containing an insoluble head group. This study was an initial attempt to prepare such vesicles in non-aqueous media. ${ }^{78}$ In Chapter-4, we present the gelation properties as well as morphology of PDMS-PTCDI along with a coil-rod-coil molecule, PDMS-PTCDI-PDMS; the luminescent properties of these organogels were examined with UV-Vis and fluorescent spectroscopy.

Chapter-5: In the literature, most of the studies regarding the gelation of PDIs are based on organic solvents to meet solubility criteria; hence, the study of self-assembly in aqueous media is very attractive. ${ }^{79}$ We study here the gel formation due to competitive hydrophilic and hydrophobic interactions. Jeffamine ${ }^{\circledR}$ (EO/PO copolymer) was used as the hydrophilic polymer, attached either to one side or both sides of a PDI unit. In this case, MJ-PTCDI was considered as inverse surfactant, since contrary to the usual definition of a surfactant, here the head group is hydrophobic, and the tail is hydrophilic. 
Chapter-6: Non-crystalline atactic polystyrene (a-PS) gels have been found to form spherical domains connected by networks. ${ }^{61}$ Since a-PS has a planar $\pi$-bonding side group, we investigated whether additional support to the $\pi-\pi$ interaction of the perylene units would enhance aggregation. Chapter- 6 describes the gelation properties as well as the resulting morphology of the extended $\pi$-electron system of a-PS. We showed here how the unique combination of a flexible polymer and a rigid, extended, $\pi$-electron system (PS-PTCDI-PS) could lead to interesting morphologies.

Chapter-7: Incorporation of functional molecules in polymers is a well-known method for devices that use films; however, small molecules do not form strong or stable films. In chapter-7, we dispersed the gelator compounds used in chapter 6, PS-PTCDI-PS, in their corresponding polymers (PS) in an attempt to make composite polymer films. Further research on these composite polymer films will shed light on their outstanding optical properties due to the presence of the perylene units. In this chapter, we proposed some future research plan for investigating the mechanical and barrier properties from these unique polymer-polymer composite interactions among the compatible polymers used.

In the overall work presented in this thesis, methods such as X-ray diffraction, optical microscopy, scanning electron and transmission electron microscopy (SEM and TEM), differential scanning calorimetry (DSC), and infra-red (IR), UV-Vis, and fluorescence spectroscopies were used to analyze the structure, morphology, and luminescent properties of the prepared gels. 


\subsection{References}

1. Saenger, W. Principles of Nucleic Acid Structure. Springer Advanced Texts in Chemistry. Springer-Verlag: New York, 1984.

2. Cantor, C.R.; Schimmel, P.R. Biophysical Chemistry, Vols. I-III. W.H. Freeman and Co.: San Francisco, 1980. pp. 1109-1264.

3. Brion, P; Westhof, E. Hierarchy and Dynamics of RNA Folding. Annu Rev Biophys Biomol Struct. 1997, 26, 113-137.

4. (a) Vögtle, F. Suparmolecular Chemistry; John Wiley \& Sons: New York, 1991. (b) Hecht, S. Construction with Macromolecules. Materials Today 2005, 8(3), 48-55.(c) Zang, L.; Che, Y.; Moore, J.S. One-dimensional Self-assembly of Planar $\pi$-conjugated Molecules: Adaptable Building Blocks for Organic Nanodevices. Acc. Chem. Res. 2008, $41,1596-1608$.

5. Hoeben, F.M.; Jonkheijm, P; Meijer, E; Schenning, A.J. About Supramolecular Assemblies of $\pi$-conjugated Systems. Chem. Rev. 2005, 105, 1491.

6. Barbara, P. F.; Gesquiere, A. J.; Park, S. J.; Lee, Y. J. Single Molecule Spectroscopy of Conjugated Polymers. Accounts Chem. Res. 2005, 38, 602.

7. Struijk, C. W.; Sieval, A. B.; Dakhorst, J. E. J.; van Dijk, M.; Kimkes, P.; Koehorst, R. B. M.; Donker, H.; Schaafsma, T. J.; Picken, S. J.; van de Craats, A. M. Liquid Crystalline Perylene Diimides: Architecture and Charge Carrier Mobilities. J. Am. Chem. Soc. 2000, $122,11057-11066$.

8. Dimitrakopoulos, C.D.; Malenfant, P.L. Organic Thin Film Transistors for Large Area Electronics. Adv. Mater. 2002, 14, 99-117. 
9. Lee, S.K.; Zu, Y.; Herrmann, A.; Geerts, Y.; Mllen, K.; Bard, A.J. Electrochemistry, Spectroscopy and Electrogenerated Chemiluminescence of Perylene, Terrylene, and Quaterrylene Diimides in Aprotic Solution J. Am. Chem. Soc. 1999, 121, 3513-3520.

10. Quante, H.; Müllen, K. Quaterrylenebis(dicarboximides). Angew. Chem., Int. Ed. Engl. $1995,34,1323-1325$.

11. Würthner, F.Plastic Transistors Reach Maturity for Mass Applications in Microelectronics. Angew. Chem., Int. Ed. 2001, 40, 1037-1039.

12. Law, K.Y. Organic Photoconductive Materials: Recent Trends and Developments. Chem. Rev.1993, 93, 449-486.

13. (a) Schmidt-Mende, L.;Fechtenkötter, A.; Müllen, K.; Moons, E.; Friend, R.H.; MacKenzie, J.D. Self-Organized Discotic Liquid Crystals for High-Efficiency Organic Photovoltaics. Science 2001, 293, 1119-1122. (b) Yakimov, A.; Forrest, S.R. High Photovoltage Multiple-heterojunction Organic Solar Cells Incorporating Interfacial Metallic Nanoclusters. Appl. Phys. Lett. 2002, 80, 1667-1669.

14. Geissler, G.; Remy, H. Ger. Pat. Appl., DE 1130099, 1959 (Chem. Abstr., 1962, 57, P11346f).

15. O'Neil, M.P.; Gaines, G.L.; Niemczyk, M.P.; Svec, W. A.; Gosztola, D.; Wasielewski, M. R. Picosecond Optical Switching Based on Biphotonic Excitation of an Electron Donor-Acceptor-Donor Molecule. Science 1992, 257, 63-66.

16. Prathapan, S.; Yang, S.I.; Seth, J.; Miller, M.A.; Bocian, D.F. Holten, D.; Lindsey, J.S. Synthesis and Excited-State Photodynamics of Perylene-Porphyrin Dyads 1.Parallel Energy- and Charge-Transport via a Diphenylethyne Linker. J. Phys. Chem. B. 2001, $105,8237-8248$. 
17. Serin, J.M.; Brousmiche, D.W.; Frechet, J.M.J. Cascade Energy Transfer in a Conformationally Mobile Multichromophoric Dendrimer. Chem. Commun. 2002, 26052607.

18. (a) Sadrai. M.; Bird, G.R. A New Laser Dye with Potential for High Stability and a Broad Band of Lasing Action: Perylene-3,4,9,10-tetracarboxylic acid-bis-N,N'(2',6' xylidyl)diimide. Opt. Commun.1984, 51, 62-64. (b) Lohmannsroben, H.G. and Langhals, H. Laser Performance of Perylenebis(Dicarboximide) Dyes with Long Secondary Alkyl Chains. Appl. Phys. B. 1989, 48, 449-452. (c) Reisfeld, R.; Seybold, G. Solid State Tunable lasers in the visible based on Luminescent Photoresistant Heterocyclic Colorants. Chimia. 1990, 44, 295-297.

19. Ford, W.E.; Kamat, P.V. Photochemistry of 3, 4, 9, 10-Perylenetetracarboxylic Dianhydride Dyes. 3. Singlet and Triplet Excited-state Properties of the Bis(2,5-di-tertbutylphenyl)imide Derivative. J. Phys. Chem. 1987, 91, 6373-6380.

20. (a)Seybold, G.; Wagenblast, G. New Perylene and Violanthrone Dyestuffs for Fluorescent Collectors. Dyes Pigm. 1989, 11, 303-317. (b) Seybold, G.; and Stange, A. (BASF AG), Ger. Pat., DE 3545 004, 1987 (Chem. Abstr. 1988, 108, 77134(c)).

21. Gvishi, R.; Reisfeld, R.; Burshtein, Z. Spectroscopy and Laser Action of the "Red Perylimide Dye" in Various Solvents. Chem. Phys. Lett. 1993, 213, 338-344.

22. (a) Mais, S; Tittel, J; Basche, T; Brauchle, C; Gohde, W; Fuchs, H; Muller, G; Müllen, K. Terrylenediimide: A Novel Fluorophore For Single-Molecule Spectroscopy and Microscopy from 1.4 K to Room Temperature. J. Phys. Chem. A. 1997, 101, 8435-8440. (b) Langhals, H.; Jaschke, H.; Ring, U.; von Unold, P. Imidazolo Perylene Imides: A 
Highly Fluorescent and Stable Replacement of Terrylene. Angew. Chem. 1999, 111, 143145.

23. Langhals, H. Cyclic Carboxylic Imide Structures as Structure Elements of High Stability. Novel Developments in Perylene Dye Chemistry. Heterocycles. 1995, 40, 477-500.

24. Langhals, H.; Demmig, S.; Potrawa,T. The Relation between Packing Effects and Solid State Fluorescence of Dyes. J. Prakt. Chem. 1991, 333, 733-748.

25. Würthner, F. Perylene Bisimide Dyes as Versatile Building Blocks for Functional Supramolecular Architectures. Chem. Commun. 2004, 14, 1564-1579.

26. (a) Graser, F.; Hädicke, E. Crystal Structure and Color of Perylene-3, 4: 9, 10bis(dicarboximide) Pigments. Liebigs Ann. Chem. 1980, 1994-2011; (b) Graser, F.; Hädicke, E. Liebigs Ann. Chem. 1984, 483-494; (c). Hädicke, E.; Graser, F. ActaCrystallogr., Sect. C 1986, 42, 189-195; (d) Hädicke, E.; Graser, F. ActaCrystallogr., Sect. C 1986, 42, 195-198; (e) Klebe, G.; Graser, F.; Hädicke, E.; Berndt, J. Acta Crystallogr., Sect. B 1989, 45, 69-77.

27. Zugenmaier, P.; Duff, J.; Bluhm, T. L. Crystal and Molecular Structures of Six Differently with Halogen Substituted Bis (benzylimido) perylene. Cryst. Res. Technol. 2000, 35, 1095-1115.

28. Kazmaier, P. M.; Hoffmann, R. J. A Theoretical Study of Crystallochromy. Quantum Interference Effects in the Spectra of Perylene Pigments. Am. Chem. Soc. 1994, 116, 9684-9691.

29. Würthner, F. Perylene Bisimide Dyes as Versatile Building Blocks for Functional Supramolecular Architectures. Chem.Commun. 2004, 14, 1564-1579. 
30. Wang, H.; Peng, B.; Wei, W. Solar Cells Based on Perylene Bisimide Derivatives. Progress Chem. 2008, 20, 1751-1760.

31. Gómez, R.; Veldman, D.; Blanco, R.; Janssen, R. A. J. Energy and Electron Transfer in a Poly(fluorene-alt-phenylene) Bearing Perylene diimides as Pendant Electron Acceptor Groups. Macromolecules 2007, 40, 2760-2772.

32. Würthner, F.; Thalacker, C.; Diele, S.; Tschierske, C. Fluorescent J-type Aggregates and Thermotropic Columnar Mesophases of Perylene Bisimide Dyes. Chem. Eur. J. 2001, 7, 2245-2253.

33. Nishinari, K. Some thought on the definition of gel. Progr Colloid Polym Sci. 2009, 136, $87-94$.

34. (a)Wu S.; Gao, J.; Emge, T. J.; and Rogers, M. A. Influence of Solvent on The Supramolecular Architectures in Molecular Gels. Soft Matter2013, 9, 5942-5950. (b) Kiyonaka, S.; Shinkai, S.; Hamachi, I. Combinatorial Library of Low Molecular-Weight Organo- and Hydrogelators Based on Glycosylated Amino Acid Derivatives by SolidPhase Synthesis. Chem.Eur. J. 2003, 9, 976-983.

35. Fages, F.; Vögtle, F.; Zinic, M. Systematic Design of Amide- and Urea- Type Gelators with Tailored Properties. Top. Curr. Chem. 2005, 256, 77-131.

36. Araki, K.; Yoshikawa, I. Nucleobase-containing Gelators Top. Curr. Chem. 2005, 256, 133-165.

37. Zinic, M.; Vogtle, F.; Fages, F. Cholesterol-based Gelators. Top. Curr. Chem.2005, 256, $39-76$.

38. Smith, D. K. Dendritic Gels - Many Arms Make Light Work. Adv. Mater. 2006, 18, 2773-2778. 
39. Kuwahara,T.; Nagase, H.; Endo, T.; Ueda, H.; Nakagak, M. Crystal Structure of DL-12hydroxystearic acid. Chem. Lett. 1996, 25, 435-436.

40. Lam, R.; Pederson, T.; Quaroni, L.; Rogers, M.A. A Molecular Insight into the Nature of Crystallographic Mismatches in Self-Assembled Fibrillar Networks Under NonIsothermal Crystallization Conditions. Soft Matter. 2010, 6, 404-408.

41. Li, J.L.; Liu, X.Y.; Wang, R.Y.; Xiong, J.X. Architecture of a Biocompatible Supramolecular Material by Supersaturation-driven Fabrication of its Network, J. Phys. Chem. B. 2005, 109, 24231-24235.

42. Rogers, M.A.; Bot, A.; Lam, R.S.H.; Pedersen, T.; May, T. Multi-component Hollow Tubules Formed Using Phytosterol and $\gamma$-oryzanol-based Compounds: An Understanding of their Molecular Embrace. J. Phys. Chem. B. 2010, 114, 8278-8295.

43. Moffat, J.R.; Smith, D.K. Controlled Self-Sorting in the Assembly of 'Multi-Gelator ' Gels. Chem. Commun. 2009, 316-318.

44. Terech, P; Furman, I.; Weiss, R.G. Structures of Organogels Based upon Cholesteryl 4(2-Anthryloxy) butanoate, a Highly Efficient Luminescing Gelator: Neutron and X-ray Small-Angle Scattering Investigations. J. Phys. Chem. 1995, 99(23), 9558-9566.

45. Toro-Vazquez, J.F.; Morales-Rueda, J.; Mallia, VA; Weiss, R.G. Relationship Between Molecular Structure and Thermomechanical Properties of Candelilla Wax and Amides Derived from (R)-12-Hydroxystearic Acid as Gelators of Safflower Oil. Food Biophys. 2010, 5, 19-202.

46. Mallia,V.A.; Butler, P. D.; Sarkar, B.; Holman, K. T.; Weiss, R. G. Reversible Phase Transitions Within Self-Assembled Fibrillar Networks of (R)-18-(n- 
alkylamino)octadecan-7-ols in Their Carbon Tetrachloride Gels. J. Am. Chem. Soc. 2011, $133,15045-15054$.

47. Weiss, R.G.; Terech, P. Introduction, in Molecular Gels: Materials with Self-Assembled Fibrillar Networks. Springer, Dordrech: The Netherlands, 2006, pp. 1-13.

48. George, M.; Weiss, R.G. Molecular Organogels. Soft Matter Comprised of Low Molecular-Mass Organic Gelators and Organic Liquids. Acc. Chem. Res. 2006, 39, 489497.

49. Zhu, G.; Dordick, J.S. Solvent Effect on Organogel Formation by Low Molecular Weight Molecules. Chem. Mater.2006, 18, 5988-5995.

50. Gao, J.; Wu, S.; Rogers, M.A. Harnessing Hansen Solubility Parameters to Predict Organogel Formation. J. Mater. Chem. 2012, 22, 12651-12658.

51. Suzuki, M.; Nakajima, Y.; Yumoto, M.;Kimua, M.; Shirai, H.; Hanabusa, K. Effects of Hydrogen Bonding and van der Waals Interactions on Organogelation Using Designed Low-Molecular-Weight Gelators and Gel Formation at Room temperature. Langmuir 2003, 19(21), 8622-8624.

52. Rogers, M.A.; Marangoni, A.G. Solvent-Modulated Nucleation and Crystallization Kinetics of 12-Hydroxystearic Acid: A Non-isothermal Approach. Langmuir 2009, 25(15), 8556-8566.

53. Raynal, M.; Bouteiller, L. Organogel formation rationalized by Hansen solubility parameters. Chem. Commun. 2011, 47, 8271-8273.

54. Hardy, J.G.; Hirst, A.R.; Smith, D.K. Exploring Molecular Recognition Pathways in Oneand Two-Component Gels Formed by Dendritic Lysine-Based Gelators. Soft Matter. 2012, 8, 3399-3406. 
55. Edwards, W.; Lagadec, C.A.; D. K. Smith, D.K. Solvent-Gelator Interactions- Using Empirical Solvent Parameters to Better Understand the Self-Assembly of Gel-Phase Materials. Soft Matter. 2011, 7, 110-117.

56. Guenet, J.M. Thermo-reversible Gelation of Polymers and Biopolymers. London: Academic Press, 1992.

57. Guenet, J.M. Polymer Thermo-reversible Gels vs. Organogels. Macromol Symp. 2006, $241,45-50$.

58. Terech, P.; Weiss, R.G. Low Molecular Mass Gelators of Organic Liquids and the Properties of their Gels. Chem. Rev. 1997, 97, 3133-3159.

59. (a) Guenet, J. M.; Wittmann, J. C.; Lotz, B. Macromolecules 1985, 18, 420. (b) Sundararajan, P. R.; Tyrer, N.; Bluhm, T. Macromolecules 1982, 15, 286. (c) Atkins, E. D. T.; Isaac, D. H.; Keller, A.; Miyasaka, K. J. Polym. Sci., Polym. Phys. Ed. 1977, 15, 211. (d) Girolamo, M.; Keller, A.; Miyasaka, K.; Overbergh, N. J. Polym. Sci., Polym. Phys. Ed. 1976, 14, 39. (e) Atkins, E. D. T.; Isaac, D. H.; Keller, A. J. Polym. Sci., Polym. Phys. Ed. 1980, 18, 71. (f) Wellinghoff, S.; Shaw, J.; Baer, E. Macromolecules 1979, 12, 932. (g) Luo, Y.; Kayakabe, S.; Xi, Y.; Bin, Y.; Xu, C.; Masaru Matsuo, M. J. Polym. Sci. Part B: Polym. Phys. 2011, 49, 384.

60. Guenet, J.M. Thermo-reversible Gelation of Polymers and Biopolymers. Academic Press: London, 1992.

61. Hikmet, R. M.; Callister, S.; Keller, A. Thermo-reversible Gelation of Atactic Polystyrene: Phase Transformation and Morphology. Polymer 1988, 29, 1378.

62. Tan, H.M.; Chang, B.H.; Baer, E.; Hiltner, A. Relationship between Crystallinity and Thermo-reversible Gelation. European Polymer Journal 1983, 19 (10), 1021-1025. 
63. Flory, P. J. Introductory Lecture. Faraday Discuss. Chem. Soc. 1974, 57, 7-18.

64. Keller, A. Faraday Discuss. 1995, 101, 1.

65. Terech, P.; Weiss, R.G. Low Molecular Mass Gelators of Organic Liquids and the Properties of Their Gels .Chem. Rev. 1997, 97, 3133-3159.

66. van Esch, J.H.; Feringa, B.L. New Functional Materials Based on Self-Assembling Organogels: From Serendipity towards Design. Angew. Chem. Int. Ed. 2000, 39, 22632266.

67. Abdallah, D.J.; Weiss, R.G. Organogels and Low Molecular Mass Organic Gelators. Adv. Mater. 2000, 12, 1237-1247.

68. Maeda, H. Anion-Responsive Supramolecular Gels. Chem. Eur. J. 2008, 14, 1127411282.

69. Krieg, E.; Shirman, E.; Weissman, H.; Shimoni, E.; Wolf, S.; Pinkas, I.; Rybtchinski, B. Supramolecular Gel Based on a Perylene Diimide Dye: Multiple Stimuli Responsiveness, Robustness, and Photofunction. J. Am. Chem. Soc. 2009, 131, 14365-14373.

70. Sugiyasu, K.; Fujita, N.; Shinkai, S. Visible-Light-Harvesting Organogel Composed of Cholesterol-Based Perylene Derivatives. Angew. Chem. 2004, 116, 1249-1253.

71. Würthner, F.; Hanke, B.; Lysetska, M.; Lambright, G.; Harms, G.S. Gelation of a Highly Fluorescent Urea-Functionalized Perylene Bisimide Dye. Org. Lett. 2005, 7, 967 -970.

72. Yagai, S.; Monma, Y.; Kawauchi, N.; Karatsu, T.; Kitamura, A. Supramolecular Nanoribbons and Nanoropes Generated from Hydrogen-Bonded Supramolecular Polymers Containing Perylene Bisimide Chromophores. Org. Lett. 2007, 9, 1137-1140. 
73. Würthner, F.; Li, X.Q.; Zhang, X.; Ghosh, S. Highly Fluorescent Lyotropic Meso phases and Organogels Based on J-Aggregates of Core-Twisted Perylene Bisimide Dyes. Chem. Eur. J. 2008, 14, 8074- 8078.

74. Dimitrakopoulos, C. D.; Malenfant, P. R. L.Organic Thin Film Transistors for Large Area Electronics. Adv. Mater. 2002, 14, 99-117.

75. Gregg, B.A. Evolution of Photophysical and Photovoltaic Properties of PeryleneBis(phenethylimide) Films upon Solvent Vapor Annealing. J. Phys. Chem. 1996, $100,852$.

76. Hoeben, F.M; Jonkheijm, P; Meijer, E.W.; Schenning, P. About Supramolecular Assemblies of $\pi$-Conjugated Systems. Chem. Rev. 2005, 105, 1491-1546.

77. Yang, X.; Xu, X.; Ji, H.F. Solvent Effect on the Self-Assembled Structure of an Amphiphilic Perylene Diimide Derivative. J. Phys. Chem. B. 2008, 112, 7196-7202.

78. Yao, D.; Bender, T. P.; Sundararajan, P. R. Self-Assembled Vesicular Nanostructures of Perylene End-Capped Poly(dimethylsiloxane). Macromolecules 2005, 38, 6972-6978.

79. Islam, R. M.; Dahan, E.; Saimani, S.; Sundararajan, P. Preclusion of Nano Scale SelfAssembly in Block-Selective Non-Aqueous Solvents for Rod-Coil and Coil-Rod-Coil Macromolecular Surfactants Based on Perylene Tetracarboxylic Diimide. European Polymer Journal 2012, 48, 1538-1554. 


\section{CHAPTER 2}

Instruments and Methods 


\subsection{Introduction}

This chapter describes the characterization methods used in this thesis and the principle of operation of the various techniques. These include infrared spectroscopy (IR), nuclear magnetic resonance spectroscopy (NMR), differential scanning calorimetry (DSC), X-ray diffraction (XRD), optical microscopy (OM), confocal scanning microscopy (CLSM), scanning electron microscopy (SEM), transmission electron microscopy (TEM), and UV-visible and fluorescence spectroscopy. Rheology and molecular modeling with HyperChem software are also introduced in this chapter.

\subsection{Instruments and Methods}

\subsubsection{Infrared (IR) Spectroscopy}

Infrared spectroscopy is one of the most often used spectroscopic tools for the study of characterization of materials. IR spectroscopy measures the vibrational energy level of molecules. The characteristic band parameters measured in IR spectroscopy are frequency (energy), intensity, band shape (environments of bonds), and the polarization of various modes, that is, the transition-moment direction in the molecular framework. ${ }^{1-4}$ The position of an infrared absorption band is specified in frequency units by its wavenumber measured in reciprocal centimeter $\left(\mathrm{cm}^{-1}\right)$, or by wavelength measured in nanometer $(\mathrm{nm})$.

Absorption of infrared radiation causes transitions between vibrational energy levels of a molecule, which are also accompanied by several rotational motions. Vibrational energy corresponds to the vibrations of atoms about the mean center of their chemical bonds, whereas rotational energy changes occur due to tumbling motion of a molecule ${ }^{1-4}$. In order to absorb IR radiation, a molecule must undergo a net change in dipole moment as a consequence of its 
vibration. Homonuclear diatomic molecules do not absorb IR radiation. On the other hand, complex mixtures give rise to very crowded spectra in which assigning of bands to specific functional groups is very difficult. Therefore, IR Spectrometry is mostly useful for pure substances ${ }^{1}$.

In an IR spectrum, the IR region usually is represented as wavenumber (in $\mathrm{cm}^{-1}$ ) vs. percent transmittance $(\% \mathrm{~T}) \cdot{ }^{1-4}$ Transmittance is the ratio of the radiant power $(\mathrm{P})$ transmitted by a sample to the radiant power incident on the sample $\left(\mathrm{P}_{0}\right)$ :

$$
\mathrm{T}=\mathrm{P} / \mathrm{P}_{0}
$$

Transmittance $(\mathrm{T})$ is related with absorbance $(\mathrm{A})$ as:

$$
A=-\log T
$$

There are two types of molecular vibrations, stretching and bending. Stretching vibration corresponds to the movement along the bond axis, which causes changes in the interatomic distance. Bending motion, on the other hand, corresponds to the changing of bond angles. Three things, number, positions and the intensity of absorption bands need to be considered while examining an IR spectrum resulted from a molecule. The theoretical number of bands, i.e. fundamental vibrations, corresponds for a molecule can be calculated from the formulas $3 \mathrm{~N}-6$ and $3 \mathrm{~N}-5$ for both non-linear and linear molecules, respectively. The formulas are derived based on the model that a molecule has three degrees of freedom on the $\mathrm{x}, \mathrm{y}$ and $\mathrm{z}$ coordinates, and molecules with $\mathrm{N}$ atoms have $3 \mathrm{~N}$ degrees of freedom. ${ }^{3}$ However, in a non-linear molecule three of these motions translate the whole molecule on the $\mathrm{x}, \mathrm{y}$ and $\mathrm{z}$ axes, while another three rotate the molecule on these axes, leaving $3 \mathrm{~N}-6$ motions for vibrational changes. On the other hand, for a linear molecule rotation around one of these axes does not affect the molecule and it has $3 \mathrm{~N}-5$ degrees of freedom for vibrations. The spectrum of a molecule may contain fewer or more bands 
than expected. The reason for having fewer bands is due to the degenerate vibrations, resulted from the absorptions of common groups at the same wavenumber. ${ }^{1}$ In addition, some of the fundamental bands can be too weak to be observed or so close that they coalesce. More bands can be seen when overtones, multiples of a given frequency, and combination bands, differences or sums of two other vibrations, are accompanied with the spectrum. The position of absorption bands depends on the relative masses of the atoms, force constants of the bonds and geometry of the atom, and these can be calculated from the Hooke's law. ${ }^{1-4}$ In practice, however, specific groups do not absorb at a definite wavenumber due to the effects of local environment of the group, they represent a broad band spreading over a range of wavenumbers. ${ }^{1}$

The IR spectrum can be divided into two regions - group frequency region (4000 to $\left.1400 \mathrm{~cm}^{-1}\right)$ and finger-print region $\left(600 \mathrm{~cm}^{-1}\right.$ to $\left.1400 \mathrm{~cm}^{-1}\right)$ (Figure 2.1). The region from 4000 to $1400 \mathrm{~cm}^{-1}$ gives information about the functional group present in the structure. Many functional groups absorb infrared radiation at about the same wavenumber, regardless of the structure of the rest of the molecule. For example, $\mathrm{C}-\mathrm{H}$ stretching vibrations usually appear between 3200 and $2800 \mathrm{~cm}^{-1}$ and carbonyl $(\mathrm{C}=\mathrm{O})$ stretching vibrations usually appear between 1800 and $1600 \mathrm{~cm}^{-1}$. The region from $600 \mathrm{~cm}^{-1}$ to $1400 \mathrm{~cm}^{-1}$ is called the fingerprint region because the pattern of absorption in this region is unique to any particular compound. ${ }^{2}$ The bands in this region can be used with great utility to assign the substitution pattern. ${ }^{3}$ 


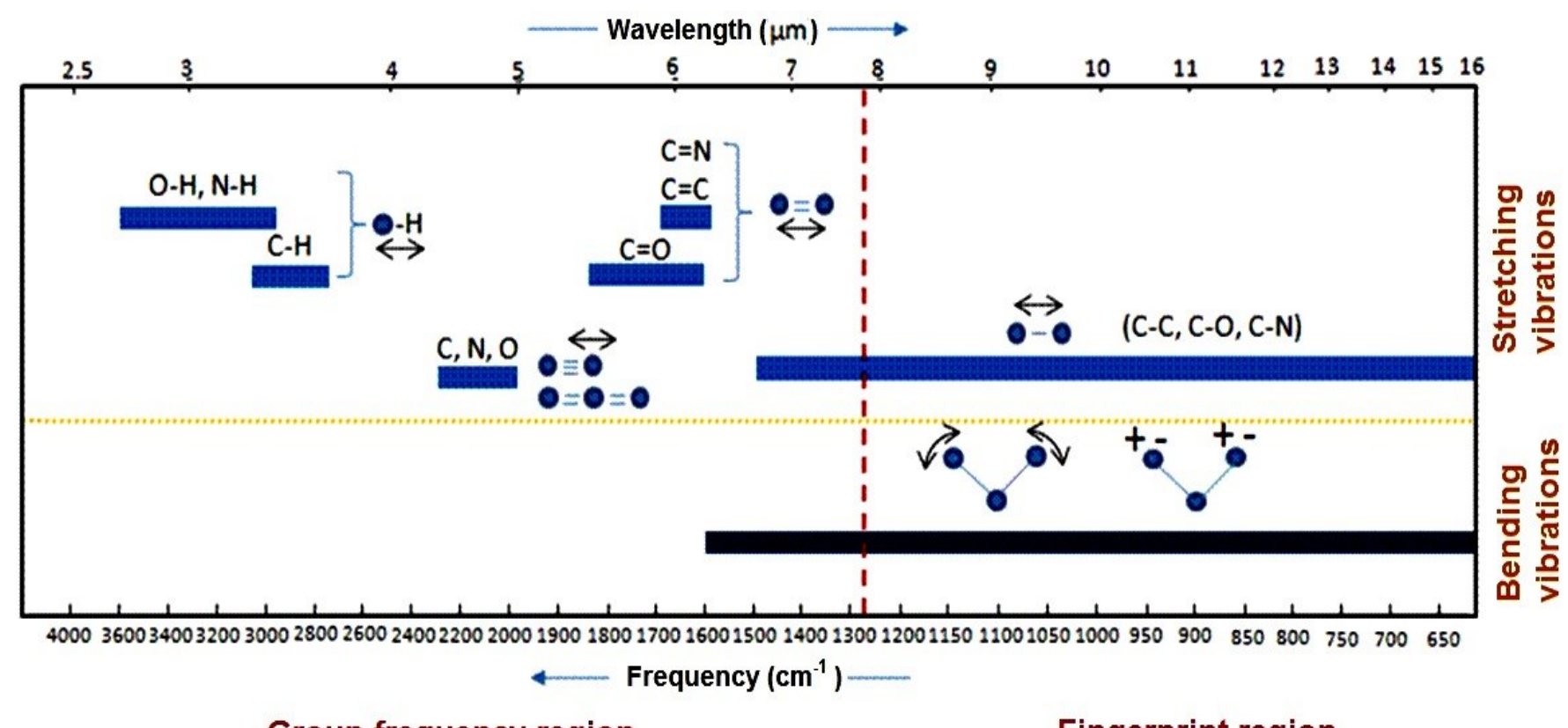

Group frequency region

Fingerprint region

Figure 2.1 Group frequency and fingerprint regions of the mid-infrared spectrum [from $\mathrm{Smith}^{5}$ ]

In the case of polymers, vibrational spectroscopy may differ from that of small molecules. ${ }^{6,7}$ The foremost difference is that although a polymer molecule often consists of many thousands of atoms, the molecule does not observe the $(3 \mathrm{~N}-6)$ discrete vibrations expected for a small molecule. Instead, for a regular polymer chain, only vibrations corresponds to the repeat units with specific phases give IR fundamental bands, and this greatly reduces the spectral complexity. In general, the interpretation of a polymer spectrum can be made on several levels. The simplest approach treats a polymer as a collection of individual functional groups and attempts to assign observed bands by using group frequency correlations. This can aid identifying (fingerprinting) or quantifying of polymer composition. ${ }^{6}$ The second level correlates spectral features with specific conformations or phases, and is valuable for morphological studies. ${ }^{7}$ The third approach attempts to assign the individual bands to their symmetry species by measuring their response to polarized radiation. This aids band assignments and helps to 
distinguish possible chain structures. ${ }^{7}$ Therefore, in terms of polymer characterization, IR spectroscopy may be employed to identify unknown polymer and by means of conformational coupling effects, permit investigations of stereochemical structures, orientation and morphology of polymers. It follows that the effect of variables which produce changes in these properties such as temperature, processing parameters, annealing times etc. may also be monitored by following the changes in the vibrational spectra.

In this thesis, FT-IR spectroscopic measurements were carried out at ambient conditions using a Varian 1000 Scimitar Series spectrophotometer. The FT-IR spectra of the polymer samples were recorded using a transparent $\mathrm{KBr}$ pellet for background correction with identical sample holder. The data were processed using the Varian Resolution (version 4.0.5.009) software.

\subsubsection{Nuclear Magnetic Resonance Spectroscopy (NMR)}

Since the 1960s, NMR spectroscopy has become a major spectroscopic tool for the study of chain configuration, sequence distribution, and microstructure in material. ${ }^{8,9}$ The technique of NMR utilizes the property of spin (angular momentum and its associated magnetic moment) possessed by nuclei whose atomic number and mass number are not both even. A special class of such nuclei, namely the class of spin half nuclei include, among others, the isotopes ${ }^{1} \mathrm{H}{ }^{13} \mathrm{C},{ }^{15} \mathrm{~N}$,

${ }^{17} \mathrm{O}$, and ${ }^{19} \mathrm{~F}$.. Application of a strong static magnetic field to a material containing such spin half nuclei splits the energy level into two, representing states with spin parallel and anti-parallel to the field (Figure 2.2). Application of a second low magnitude oscillating magnetic field (radio frequency) perpendicular to the large static field causes transitions between the states through absorption or emission of energy. The energy change is observed as a resonance peak or line in 
experiments where either field strength of the static magnetic field or frequency of the excitation is varied keeping the other constant. The positions of resonance lines on the scale of frequency or magnetic field for a particular nucleus depend on the local fields created by the surrounding electron cloud, which in turn result from the nature and location of the atomic groups in the vicinity of the protons and from the nature of chemical bonds present in the molecule.

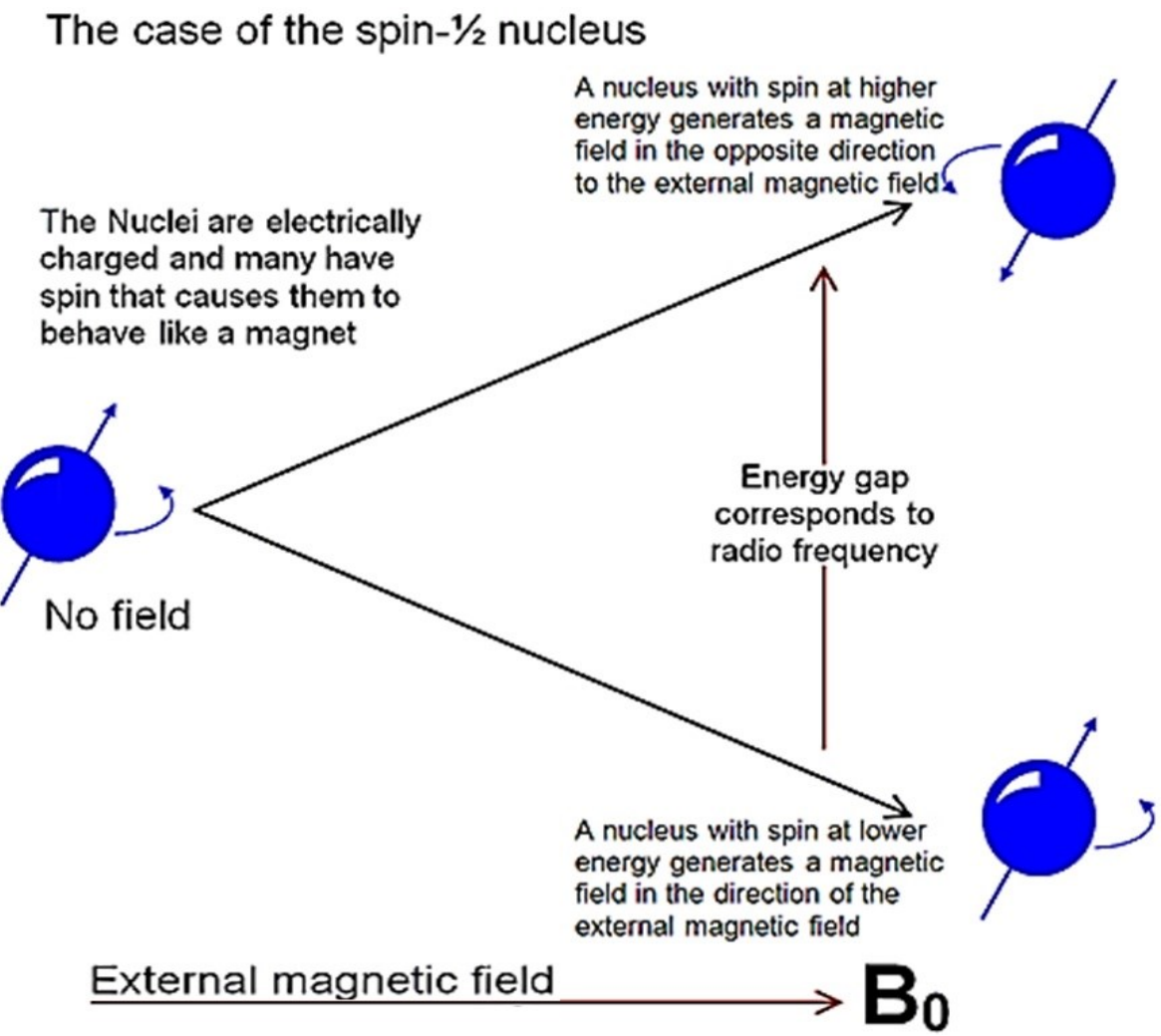

Figure 2.2 The spin-1/2 nuclei include the most common ${ }^{1} \mathrm{H}$ NMR nucleus, as well as many other nuclei such as ${ }^{13} \mathrm{C},{ }^{15} \mathrm{~N}$ and ${ }^{31} \mathrm{P}$ [from Bushong ${ }^{8}$ ]

The displacements in the resonance due to the chemical (electronic) environment, called chemical shifts, and are measured in parts per million in frequency on a scale labeled $\delta$. The zero of the $\delta$ scale is a reference point provided by the single resonance of the equivalent protons in a substance showing minimum chemical shift, such as tetramethylsilane (TMS) (Figure 2.3). 

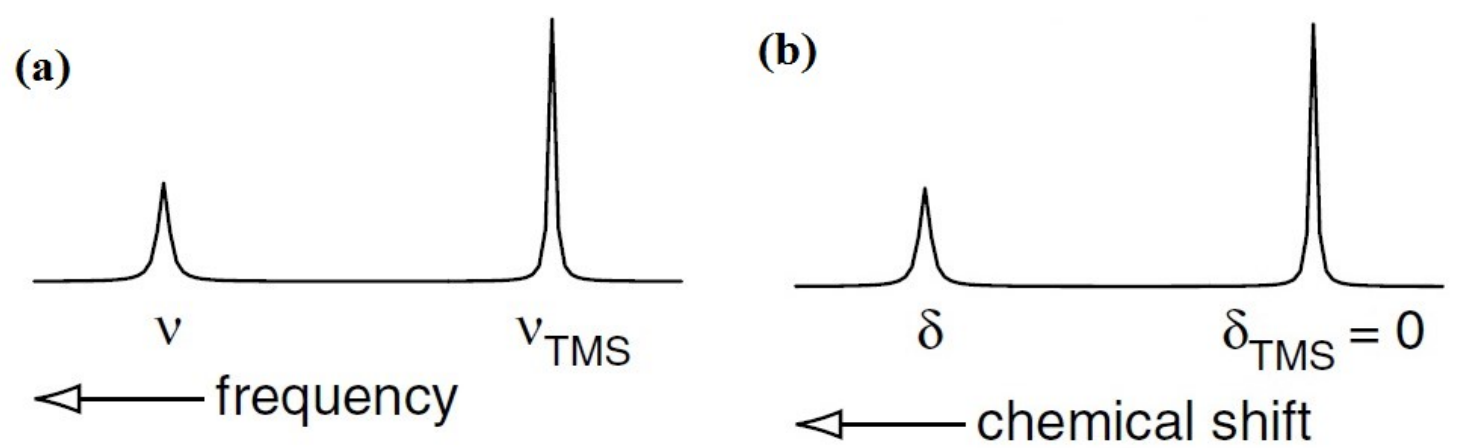

Figure 2.3 An NMR spectrum can be plotted as a function of frequency (a); however, it is best to use the chemical shift scale (b) in which frequencies are expressed relative to that of an agreed reference, such as TMS in the case of proton spectra

The actual procedure for obtaining the spectrum varies, but the simplest is referred to as the continuous wave $(\mathrm{CW})$ method for a ${ }^{1} \mathrm{H}$ NMR (Figure 2.4). A solution of the sample in a uniform $5 \mathrm{~mm}$ (diameter) glass tube is oriented between the poles of a powerful magnet, and is often spun to average any magnetic field variations, as well as tube imperfections. Radio frequency radiation of appropriate energy is broadcast into the sample from an antenna coil (colored red). A receiver coil surrounds the sample tube, and emission of absorbed radio frequency $(\mathrm{RF})$ energy is monitored by dedicated electronic devices and a computer. An NMR spectrum is acquired by varying or sweeping the magnetic field over a small range while observing the RF signal from the sample. An equally effective technique is to vary the frequency of the RF radiation while holding the static field strength constant. In proton NMR, additional complexity and additional information result from coupling of the resonances of protons on adjacent carbon atoms, resulting in the splitting of their resonances into $(n+1)$ peaks, where $n$ is the number of equivalent neighboring protons. To aid in interpretation, two experimental modifications are useful. The first of these is the use of high magnetic field strength (present NMR is usually 400-500 MHz). The second development, double resonance or spin decoupling, 
provides great simplifications of spectra. A second radiofrequency field is used that has the effect of removing the coupling and collapsing multiplet spectra to much simpler ones.

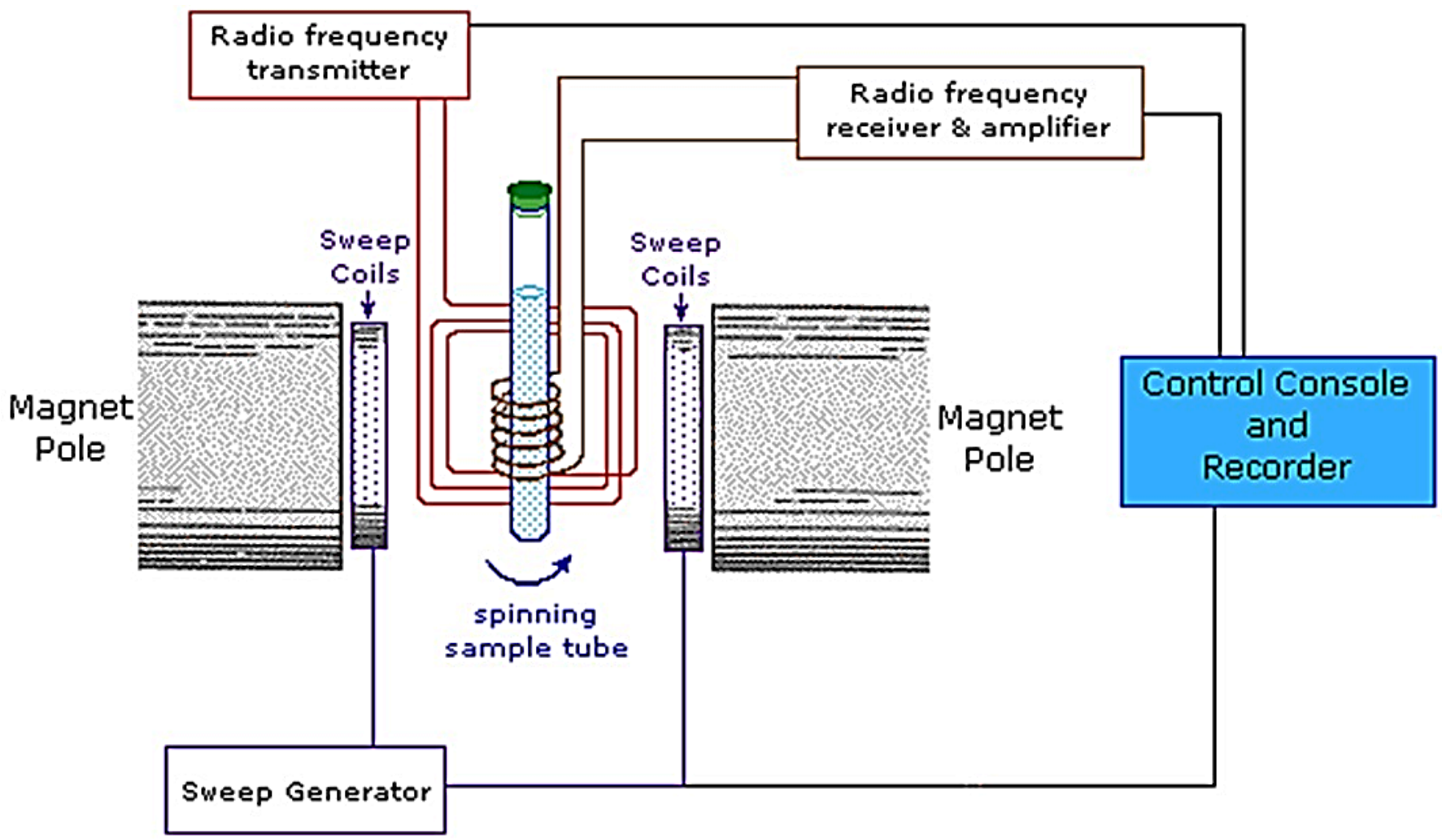

Figure 2.4 NMR spectrum acquired by varying the magnetic field over a small range while observing the RF signal from the sample [from Nyquist ${ }^{9 b}$ ]

In this thesis, a Bruker-400 instrument was used to record all ${ }^{1} \mathrm{H}$ NMR spectra, while Tetramethylsilane (TMS) was used as internal reference. 


\subsubsection{Differential Scanning Calorimetry (DSC)}

Differential scanning calorimetry is one of the most common techniques for quantitative studies of thermal transitions in polymers, organic materials, metals, ceramics and composites. In DSC, the sample and an inert reference are heated, usually in a nitrogen atmosphere for measuring the thermal transitions. ${ }^{10}$ Figure 2.5 is a schematic representation of the DSC cell. The sample and the reference are provided with individual heaters, and energy is supplied to keep the sample and reference temperatures constant (Figure 2.5). The electrical power difference between sample and reference $(\mathrm{d} \Delta \mathrm{Q} / \mathrm{dt})$ is recorded. This heat flow/temperature data provides valuable information regarding the physical/chemical properties of the materials such as the crystalline melting temperature $\left(\mathrm{T}_{\mathrm{m}}\right)$, the crystallization temperature $\left(\mathrm{T}_{\mathrm{c}}\right)$ and the heat of fusion $(\Delta \mathrm{H})$. The glass transition temperature $\left(\mathrm{T}_{\mathrm{g}}\right)$, which is a second order transition, and the specific heat capacity $\left(\mathrm{C}_{\mathrm{p}}\right)$ of a polymer can also be determined from DSC. Polymorphic transitions are also detectable. ${ }^{10}$ Among the DSC applications of polymers, glass transition temperature $\left(\mathrm{T}_{\mathrm{g}}\right)$ is one of the most important ones; generally, small molecules do not show a $\mathrm{T}_{\mathrm{g}}$. Glass transition is exhibited by amorphous polymers or the amorphous regions of partially crystalline polymers when a hard, brittle, glass-like state is transformed into a viscous or rubbery state. According to Fox and Flory's theory ${ }^{11}$, below $\mathrm{T}_{\mathrm{g}}$, the local conformational arrangement of the polymer segments is independent of both molecular weight and temperature. The glass transition temperature is a very important characteristic of a polymer. 


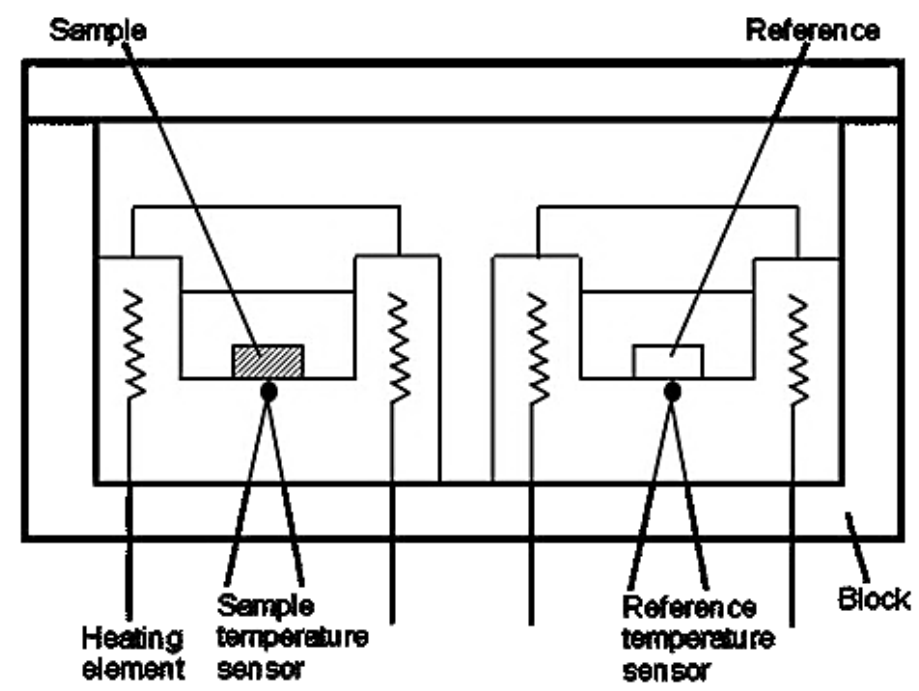

Figure 2.5 Schematic representation of DSC cell

In this study, we performed thermal analysis of our samples using a TA Instruments 2010 differential scanning calorimeter at a heating rate of $10{ }^{\circ} \mathrm{C} / \mathrm{min}$. A few selected samples were heated at a heating rate of $1{ }^{\circ} \mathrm{C} / \mathrm{min}$. The instrument was calibrated for temperature and energy with indium and tin as certified reference materials. DSC traces for gel samples were recorded with about $10-15 \mathrm{mg}$ of the samples under the flow of nitrogen. The uncertainty in the measurements was $\pm 1{ }^{0} \mathrm{C}$ for the melting and crystallization points and $\pm 2 \mathrm{~J} / \mathrm{g}$ for the heat of fusion and crystallization.

\subsubsection{X-ray Diffraction}

X-ray diffraction, a non-destructive analytical technique based on constructive interference of monochromatic X-ray, is commonly used for identification and quantitative determination of various crystalline forms or phases. Crystalline substances are composed of parallel rows of atoms separated by "unique" distance (d-spacing); diffraction occurs when electromagnetic radiation enters such a crystalline substance and is scattered from the different 
planes of that substance. The wavelengths of $\mathrm{X}$-rays are comparable to interatomic distances in crystals. ${ }^{12,13}$ Most of our knowledge regarding atomic positions and intramolecular distances is gained from X-ray diffraction measurements.. For complete information on the molecular ordering in a crystalline or semicrystalline material, it is necessary to obtain a single crystal of the compound. ${ }^{12,13}$ However, when a single crystal is not available, powder X-ray diffraction can provide valuable information regarding the interplanar distances between atomic planes (d spacing), crystallite size and crystallinity.

In powder X-ray diffraction, the reflections are obtained from several microcrystals in different orientations. This method is more convenient, but gives less information than the single crystal method. ${ }^{12,13}$ Direct methods are also available to determine the crystal structure using single crystals. The basic equation of X-ray diffraction is the Bragg formula:

$$
2 d \sin \theta=n \lambda
$$

where, $\lambda$ is the wavelength of $\mathrm{X}$-ray radiation, $\mathrm{d}$ the distance between atomic planes, $\theta$ is the angle of incidence of the x-ray beam on the atomic planes and $n(1,2,3 \ldots, n)$ is the order of the reflection. Constructive interference only occurs for certain $\theta$ values correlating to a (hkl) plane, specifically when the path difference is equal to $\mathrm{n}$ wavelengths (Figure 2.6).

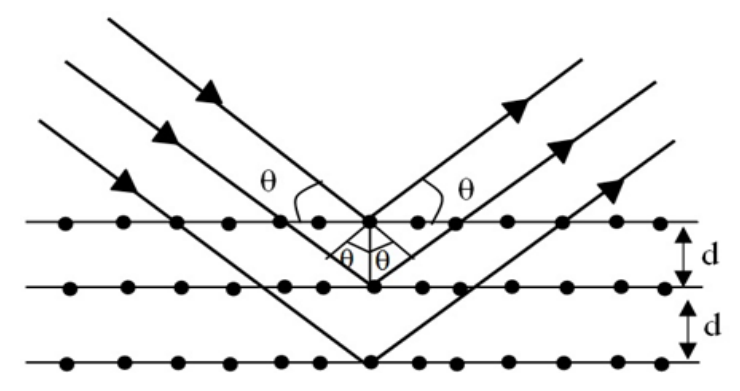

Figure 2.6 Bragg construction illustrating the principle of diffraction where $d$ is the spacing between two atomic planes 
Higher order reflections corresponding to $\mathrm{n}>1$ are extremely weak in intensity; only those corresponding to $n=1$ are observed. Hence, the above equation reduces to

$$
2 d \sin \theta=\lambda
$$

Rearrangement of Bragg's equation gives the $d$-spacing as

$$
d=\frac{\lambda}{2 \sin \theta}
$$

Equation 2.5 shows that when the distance (d) between the two planes in the lattice is large, the

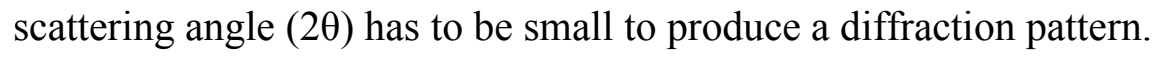

The diffractions obtained at angles smaller than $2^{0}(\mathrm{~d}>44 \AA)$ are called Small Angle Xray Diffraction (SAXD) ${ }^{13}$ The diffractions obtained at all larger angles, theoretically extending up to $180^{\circ}$ are called Wide Angle X-ray Diffraction (WAXD). ${ }^{13}$ WAXD provides information about the spatial arrangement of atoms, whereas SAXD provides information about domain arrangements (e.g. long spacing). ${ }^{13}$

In our study, X-ray diffraction data were collected within the range of $2^{0} \leq 2 \theta \leq 50^{0}$ for most of the samples using a Philips automated powder diffractometer, Model PW 1710. Nickelfiltered $\mathrm{Cu} \mathrm{K} \mathrm{K}_{\alpha}$ radiation $(\lambda=1.542 \AA)$ was used. The possible presence of texture in many samples was checked by taking additional diffractograms by orienting the samples in the plane of measurement by $90^{\circ}$. Figure 2.7 shows a schematic of the Bragg diffraction with this diffractometer. 


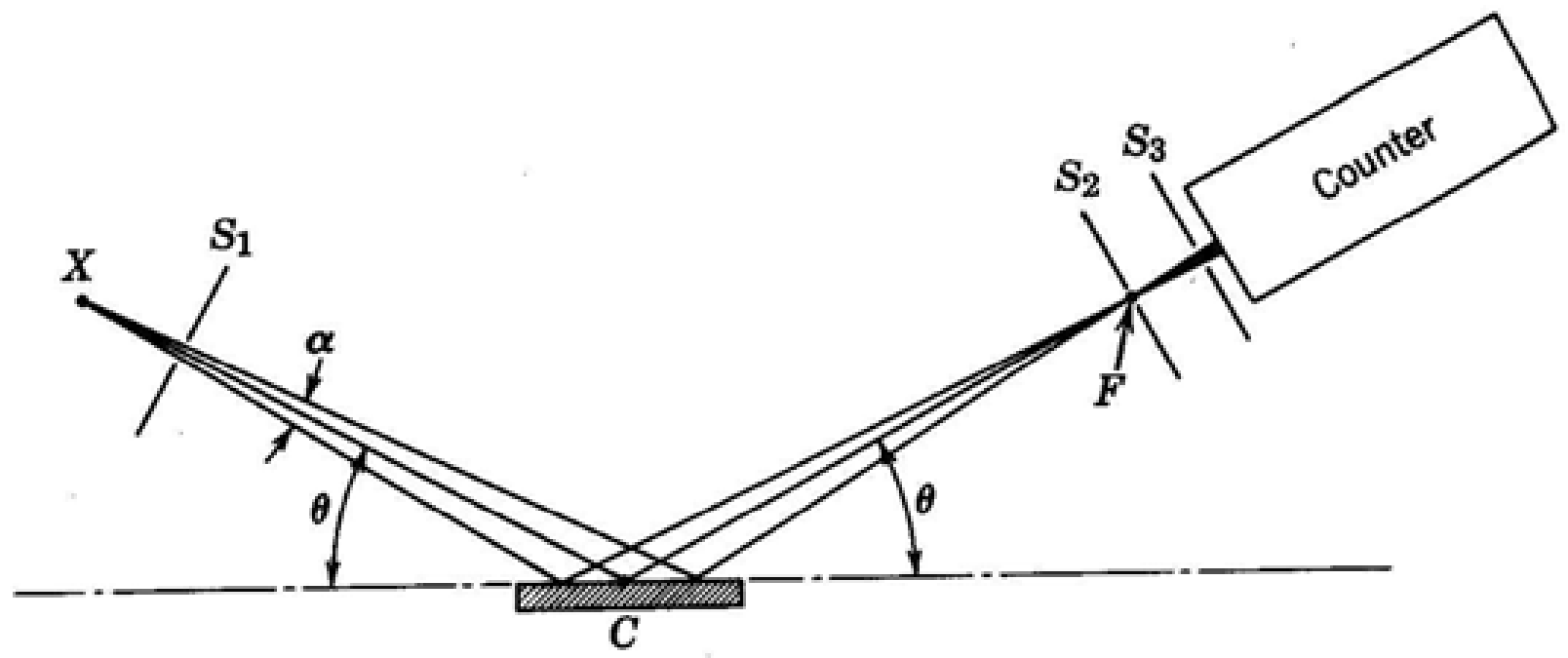

Figure 2.7 Schematic of the Bragg diffraction with the powder X-ray diffractometer [Klug ${ }^{13}$ ]. Here, $S_{1}, S_{2}$ and $S_{3}$ are the divergence slit, receiving slit and scatter slit respectively; $\alpha=1-4^{0}$

The MDI Data scan 3.2 software (Materials Data Inc., Livermore, CA) was used for data collection. The results were analyzed using MDI Jade 5.0 XRD Pattern Processing software.

\subsubsection{Optical Microscopy (OM)}

Optical microscopy is applied not only to examine more clearly the fixed and unchanging features of an object, but also to probe into it for observing the behavior or other changes that can occur in a variety of applied conditions and circumstances. An advanced instrument thus can be equipped with a range of accessories and features such as for various systems of illumination or technique including heating-stage, phase-contrast, interferometry, photomicrography, fluorescence, ultra-violet light, and polarizations. ${ }^{14}$ An optical microscope can resolve surface characteristics of the order of $2000 \AA$. Polarized light microscopy uses plane-polarized light to analyze structures that are birefringent; i.e., the structures that have two different refractive indices at right angles to one another. Normal un-polarized light can be considered as many sine 
waves, each oscillating at any one of an infinite number of orientations (planes) around the central axis. Plane-polarized light only oscillates in one plane because the polarizer only transmits light in that plane.

The polarized light microscope must be equipped with both a polarizer, positioned in the light path somewhere before the specimen, and an analyzer (a second polarizer), placed in the optical pathway after the objective rear aperture. Image contrast arises from the interaction of plane-polarized light with a birefringent (or doubly-refracting) specimen to produce two individual wave components that are each polarized in mutually perpendicular planes. The velocities of these components are different and vary with the propagation direction through the specimen. After exiting the specimen, the light components become out of phase, but are recombined with constructive and destructive interference when they pass through the analyzer.

In our study, optical microscopy was used extensively to investigate the morphology of the prepared gels and xerogels. The optical micrographs were recorded using a Zeiss Axioplan polarized optical microscope $(\mathrm{OM})$, equipped with a Linkam hot stage for variable temperature optical microscopy. All the optical micrographs were taken in transmission mode. Northern Eclipse (version 6.0 and 8.0) image processing software was used to record the images as well as to calculate (where it was possible) the size of the features, e.g., fibers, spheres, spherulites, etc.

\subsubsection{Confocal Scanning Microscopy (CLSM)}

The major optical difference between a conventional microscope and a confocal microscope is the presence of the confocal pinholes, which allow light only from the plane of focus to reach the detector ${ }^{15}$ (Figure 2.8). This forms the principle of a confocal microscope where "out of focus" light is removed from the image by the use of a suitably positioned pinhole. 
This produces images of exceptional resolution and clarity, and also allows the user to collect optical slices of the object for creating a $3 \mathrm{D}$ representation of the sample. ${ }^{15}$ The image is reconstructed serially, i.e. point by point using a single photodetector, rather than in parallel (in bright field, phase contrast, etc.), where a 2D image is recorded via a detector array, such as a camera. The illumination light is focused down to a small spot at the sample plane, while the detector records the light originating from the same spot, hence the name confocal. ${ }^{15}$ This technique involves changing the level or plane at which the sample is observed. If the plane of focus is changed, or the object moved, a series of images at different positions can be produced through the thickness of the object, i.e. a series of $\mathrm{X}-\mathrm{Y}$ images at different $\mathrm{Z}$ positions. ${ }^{15}$ Such a series of images is a three dimensional representation of the object being looked at, produced by optical sectioning. This is the main feature and a major advantage of confocal microscopy over conventional microscopy.

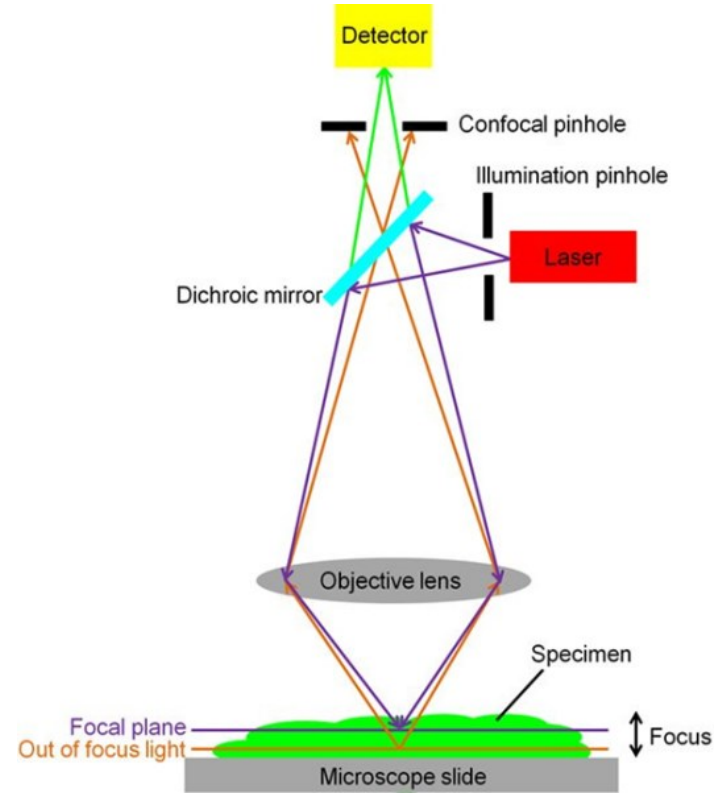

Figure 2.8 In a CLSM, with image processing, many slices can be superimposed giving an extended focus image (from Pawley ${ }^{15}$ ). This can only be achieved in conventional microscopy by reduction of the aperture and thus sacrificing resolution 
In this thesis, a confocal laser-scanning microscope was used to observe the morphology of our poly(dimethylsiloxane) (PDMS) gel samples. Confocal laser-scanning microscopy (CLSM) images were recorded at Agriculture Canada Research Laboratories. Samples were prepared by spreading a small amount of the PDMS gel on a glass slide, Fluoromount G mounting medium (Electron Microscopy Science Cat \# 17984) was added and covered with a cover slip. CLSM was performed using a Zeiss LSM 510 Duo microscope equipped with a multi-line Argon 2 laser and an Apochromat 63X Oil DIC objective (Zeiss \# 420782-9900). Excitation was done with $488 \mathrm{~nm}$, and the auto fluorescence emission was collected with a LP $505 \mathrm{~nm}$ filter. In most cases overlay projections were produced, i.e. several images were taken at different focal planes and overlays were produced with the confocal microscope software Zen 2009.

\subsubsection{Scanning Electron Microscopy (SEM)}

The need to observe, analyze, and correctly explain phenomena occurring on a micrometer or submicrometer scale is the driving force behind the development of sophisticated microscopic techniques in this era of rapidly expanding nanotechnology. The scanning election microscopy (SEM) is such a technique which permits the observation and characterization of heterogeneous organic and inorganic materials and surfaces on such a local scale. ${ }^{18}$ In SEM, the area of the sample to be examined is irradiated with a finely focused electron beam which is swept in a raster across the surface of the specimen. Upon irradiation, various types of signals are produced which includes secondary electrons, backscattered electrons, Auger electrons, characteristic X-rays and photons of various energies. In SEM, the secondary and backscattered electrons are the signals of greatest interest because of their variance as a result of differences in 
surface topography as the electron beam scans across the specimen surface. These scattered electrons are used to produce a signal that modulates the intensity of the electron beam in a cathode-ray tube, scanning in synchronization with the microscope beam. The resulting images have great depth of fields and a remarkable three-dimensional appearance ${ }^{18}$ due to the confinement of the secondary electrons to a volume near the beam impact area of the sample and the shadow relief effect of the secondary electron contrast. SEM provides useful information on surface topology with a resolution of about $100 \AA .^{17,18}$ Figure 2.9 represents the schematic of a scanning electron microscope.

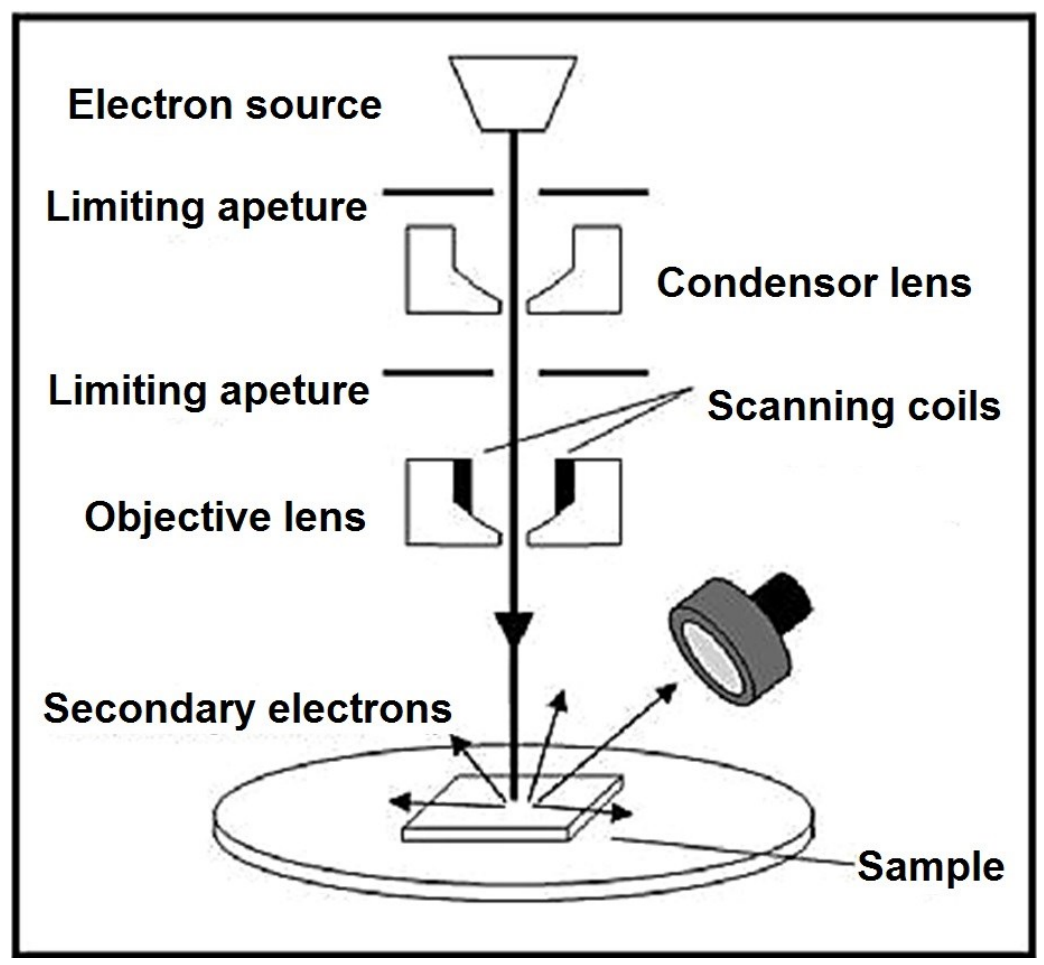

Figure 2.9 Schematic diagram of a Scanning Electron Microscope [from Kalantar-zadeh ${ }^{18}$ ]

In this study, SEM images were obtained using a JEOL JSM-6400 scanning electron microscope. Samples were prepared for SEM by mounting on carbon tape and sputter coating 
with $80: 20 \mathrm{Au} / \mathrm{Pd}$ alloy. The accelerating voltage of SEM was $15 \mathrm{kV}$. Scanning electron microscopy (SEM) images of some samples were obtained using a VEGAII XMU (TESCAN, Czech Republic) scanning electron microscope. Dried samples were sputter coated with 80:20 Au/Pd target using a Hummer VIII Sputtering System (Anatech Ltd., Alexandria, VA) before recording the images. SEM images were captured at an accelerating voltage ranging from 5 to 20 $\mathrm{kV}$. Vega TC software were used for both the microscope manipulation and capturing the images. Cryo-SEM images were recorded with a CryoTrans CT1000 system attached to a Philips XL 30 E-SEM located at Agriculture Canada Research Laboratories (courtesy to Mr. Ann-Fook Yang). The specimens were cooled in a liquid nitrogen slush $\left(-210^{\circ} \mathrm{C}\right)$ in vacuum $(3 \times 10-5$ mbar), then stage mounted and defrosted to $-80^{\circ} \mathrm{C}$ to eliminate surface moisture before cooling gradually with $\mathrm{N} 2$ to a temperature of $-140^{\circ} \mathrm{C}$, coated with gold and examined in the SEM at $20 \mathrm{kV}$.

\subsubsection{Transmission Electron Microscopy (TEM)}

TEM provides detailed structural information at levels down to atomic dimensions. Under favorable conditions, the most capable instruments can resolve detail at the $0.1 \mathrm{~nm}$ level, but such high resolution examination is seldom possible with a thick sample. ${ }^{17}$ Nevertheless, it is possible to obtain information within the range 1-100 $\mathrm{nm}$ with varying degrees of difficulty. This is beyond the range of light microscopy, and TEM can provide information that can rarely be obtained by any other means. Further advantage of the TEM is that it can be rapidly adjusted to provide an electron diffraction pattern from selected areas, facilitating investigation of crystal structure and orientation, and enabling particular morphological features to be identified. The main disadvantage of the TEM is that it can be used only on thin samples of less than $1 \mu \mathrm{m}$ 
thickness, and preferably less than $100 \mathrm{~nm}$ thickness. Problems are encountered as a direct consequence of the way in which the sample and the electron beam interact, because electron irradiation results in chemical changes, destruction of crystallinity and mass transport. ${ }^{16,17}$

In addition, the electron beam may also cause heating, which increases the beamsensitivity of the material still further. In those cases, the problems are often best overcome by the indirect examination of the sample. In effect, successful TEM depends on three skills: preparing a good specimen, acquiring good data, and adequate interpretation. TEM images are actually contrast images. ${ }^{17}$ Contrast is produced in the image if different sites within the specimen transmit electrons with different efficiencies. For example, if a very thick region of the specimen is adjacent to a very thin region, most electrons passing through the thick part will be scattered and will fail to pass through the aperture causing the corresponding part of the image to be dark, whereas a high proportion of the electrons passing through the thin area will be undeviated and will reach aperture making the image bright. This is known a "mass-thickness" contrast, because a similar effect can be seen if two regions having same thickness but containing atoms with different atomic masses transmit electrons with different efficiencies. As a consequence, i.e., the image from atom silicon (atomic weight is 28) is darker than that from atom carbon (atomic weight is 12).

In this thesis, direct examination was used for studying the samples; transmission electron micrographs were recorded using a FEI Tecnai F20 G2 FE TEM operated at 200kV. The samples were prepared by spreading a small amount of the gels onto a carbon lacey grid. The thin xerogels were formed by allowing the gels to dry for a few hours before examination. 


\subsubsection{Absorption and Emission Spectroscopy}

Outer orbital electrons of a molecule have higher energy than inner shell electrons and the energies are quantized. During the process of electron transition a photon can be absorbed when its energy corresponds to the excitation of an electron to a more energetic state, and a photon of an appropriate wavelength can be emitted as well when an excited electron drops back to a lower state. The fraction of light that absorbs during electronic transition is called absorbance and is proportional to concentration and path length. The transitions from lowest energy ground state $\left(\mathrm{S}_{0}\right)$ to excited states $\left(\mathrm{S}_{1}, \mathrm{~S}_{2}\right.$, etc......) are associated with photon wavelength of IR, visible and UV band. If the electronic transition occurs in the visible range of wavelength, we see color. The Jablonski diagram, in Figure 2.10, is a partial energy diagram that represents the energy of photoluminescent molecule in its different energy states.

Singlet Excited States

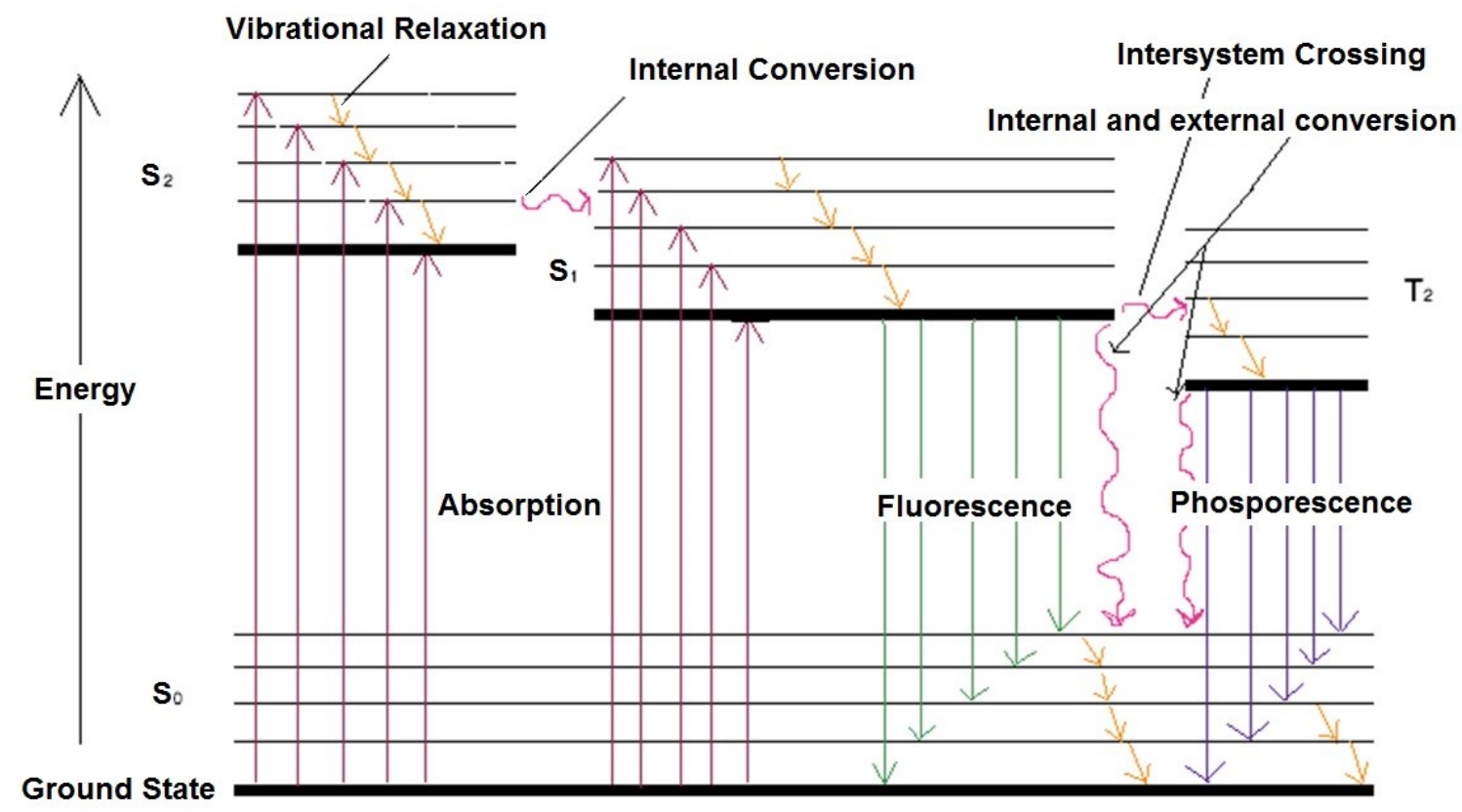

Figure 2.10 Partial Jablonski diagram for Absorption, Fluorescence, and Phosphorescence 
The upper lines represent the energy state of the three excited electronic states: $\mathrm{S}_{1}$ and $\mathrm{S}_{2}$ represent the electronic singlet state, while $T_{1}$ represents the first electronic triplet state. The upper darkest line represents the ground vibrational state of the three excited electronic state. The energy of the triplet state is lower than the energy of the corresponding singlet state. There are numerous vibrational levels that can be associated with each electronic state as denoted by the thinner lines. Absorption transitions can occur from the ground singlet electronic state $\left(\mathrm{S}_{\mathrm{o}}\right)$ to various vibrational levels in the singlet excited vibrational states.

Electronic energy levels are composed of vibrational and rotational energy levels; therefore, an electronic transition is always accompanied by vibrational and rotational level transitions. This gives a broad absorption band resulting from the superposition of vibrational and rotational transitions. The emission spectrum originates from emission of photon by excited electron to get back to the ground state and is often a mirror image of the absorption spectra (Figure 2.11).

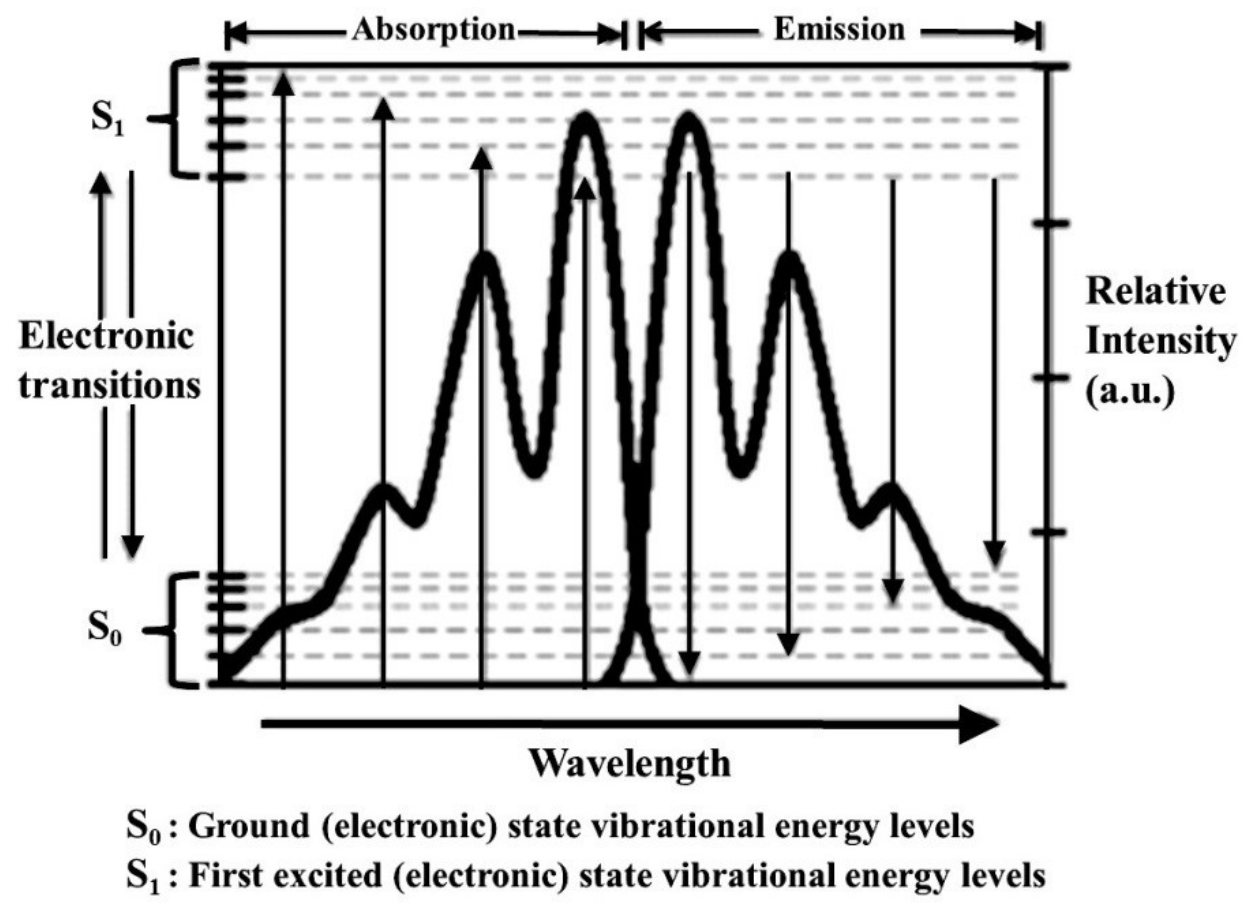

Figure 2.11 Illustration of emission spectrum as a mirror image of the absorption spectrum 


\subsubsection{UV-visible Spectroscopy}

The UV-visible spectrum corresponds to electronic excitation, and the energy levels depend on the chemical bonds within the specimen. Sigma $(\sigma)$ electrons, involved in covalent bonds, absorb high energy photons in the UV region, whereas $\pi$ electrons absorb low energy photons at longer wavelengths, often in the visible region. Although UV-visible spectroscopy is not capable alone of completely identifying an unknown compound, the advantage of using very

small samples and rapid analysis makes it still a common routine analytical technique. ${ }^{20} \mathrm{~A}$ modern application of UV-visible spectroscopy is in the analysis of rigid rod molecules with liquid crystal properties. The $\pi$-electrons in a conjugated system are delocalized, which means that they are not restricted to an atomic orbit, but can move freely within the conjugated region. The uninterrupted conjugation lengths can be obtained using UV-visible spectroscopy. The theory starts with the assumption that the behavior of a $\pi$-electron can be modeled by a particle in a square potential well with infinite side, and whose length is chosen to coincide with the conjugation length within the molecule. Such a particle can exist anywhere within the well, but it cannot escape. This represents a good approximation, because a $\pi$-electron has a very low probability of escaping.

In this study, The UV-visible absorption spectra were recorded using a Varian CARY 3 UV-Vis spectrophotometer. The path length of the quartz sample cube was $10 \mathrm{~mm}$. The data were processed by CARY WinUV Software version 3.00.

\subsubsection{Fluorescence Spectroscopy}

Fluorescence occurs when some chromophores, after being electronically excited, reemit the energy. Upon absorbing photon, the chromophore molecule is usually brought to a state that 
is excited both electronically and vibrationally. However, non-radiative relaxation very quickly brings the chromophore to its lowest vibronic level within the electronically excited state. When the remaining excitation energy is reemitted, the chromophore returns to the electronic ground state, and this is known as fluorescence emission. The molecule usually retains some vibrational energy, which is subsequently also dissipated. Thus, the fluorescent radiation occurs at longer wavelengths than the exciting radiation; a whole fluorescence spectrum is observed because the molecules may end up in different vibronic states. The reradiation process is slow as electronic phenomena; the lifetimes of the excited states are measured in nanoseconds, while most transitions among electronic states are completed in picoseconds or faster. In studies on $\pi$ conjugated systems, the chromophore is either a part of the macromolecule itself or fluorescent probes are covalently attached to the macromolecules (e.g., on chain end). Since most fluorescence occurs in a solvent, the environmental factors must be considered. ${ }^{21}$ These factors include interactions with solvent and other dissolved compounds, temperature, $\mathrm{pH}$, and the concentration of fluorescent species. The effects of these four parameters vary from fluorescent moiety to moiety. Absorption and emission spectra as well as the quantum efficiencies of fluorescent molecules are influenced by these parameters.

In this study, the fluorescent emission data were collected using a Varian CARY Fluorescence spectrophotometer at the excitation wavelengths $\left(\lambda_{\mathrm{ex}}\right)$ of $460 \mathrm{~nm}$ and $417 \mathrm{~nm}$ with a bandwidth of $5 \mathrm{~nm}$ for excitation and $5 \mathrm{~nm}$ for emission. Data collection and processing were done by Eclipse WinFLR Software (version 1.1). 


\subsubsection{Molecular Modeling}

There are three main reasons why computational chemistry is so widely used. First, it can help us to understand the behavior of a system at molecular level information that may be difficult if not impossible to obtain by any experimental technique. Second, it enables researchers to evaluate many possible choices, in terms of reactants, reaction conditions and so on, before undertaking any experimental work. Third, computational methods can be used to investigate systems under extreme conditions such as very high pressures and/or temperatureswhich cannot be reproduced in the laboratory. Easy-to-use software packages are now available based on a wide variety of ingenious computational methods; these can be used to build computer models that probe the intimate details of sample reactions, predict the characteristics of materials such as catalysts and polymers, and visualize the interactions of biological molecules and drugs.

Modeling study ranges from single isolated molecules to polymers containing thousands of atoms immersed in solvent molecules. Computational chemists have at their disposal a variety of methods but all programs are based on the procedure for calculating the energy of the system. The programs then make small changes to the system and recalculate the energy after each change. It uses the principle that a chemical system tends to adopt the lowest energy which it can attain to select the most likely configuration of the system. Energies can be calculated using two basic methods: quantum mechanics and molecular mechanics. Quantum mechanics offers the most fundamental approach, and is mostly used for simple atoms and molecules; however, it is usually not feasible to model larger molecules, or assemblies of molecules. Molecular mechanics is particularly useful for modeling large molecules and assemblies of molecules.. ${ }^{22,23}$ The molecular mechanics approach considers the energy of any arrangement of atoms to be made up 
of several distinct parts: the bond stretch (extension or compression from the "ideal" values); bond angle bend; stretch-bend interaction (to account for change in bond bending difficulty when bonds are stretched a certain amount); torsion ; and van der Waals attraction or repulsion (which is separated into interactions between atoms that are bonded to bonded atoms and more distantly connected atoms). These steric energies can be calculated separately and then added up.

HyperChem is a desktop molecular modeling system developed by HyperCube Inc. (Waterloo, Ontario). It is used to run on PC under Windows. Like most other molecular modeling programs, HyperChem is accessed through a graphical user interface with pull-down menus. It provides a molecular editor for constructing molecules on the screen or it can read molecular structure files in several formats, and it provides a variety of molecular mechanics and quantum mechanical computational methods together with various options for presenting and rendering different aspects of molecular structure, including isodensity surfaces, electron orbitals, and vibrational modes. HyperChem is more basic than Cerius ${ }^{2}$. It is more suitable for small organic molecules and natural polymers. In this thesis, simple molecular modeling was performed using HyperChem (Version 7.0) on Windows XP operation system. Geometry optimization of the molecular structures was done using the $\mathrm{MM}+$ force field.

\subsubsection{Rheology}

\subsubsection{Introduction}

Fluids can be classified as "compressible" or "incompressible" according to their response to

externally applied force or deformation. ${ }^{24-27}$ Thus the simplest relation between force and deformation is Hooke's law, where force is proportional to deformation:

$$
\sigma=\mathrm{G} \gamma
$$


where, $\sigma$ is the force per unit area or stress, $\gamma$ is the relative change of strain, and $G$ is the elastic modulus, which is an intrinsic property of a solid.

According to this, Hookean materials do not flow and are linearly elastic. Therefore, stress remains constant until the strain is removed, and the material returns to its original shape. Hooke's law can be used to describe many solids; only brittle materials are linearly elastic up to the point where they fracture. ${ }^{25,26}$

At the other end of the spectrum, Isaac Newton proposed that for fluids, the force per unit area required to produce motion is $\mathrm{F} / \mathrm{A}$ and is denoted by $\sigma$.

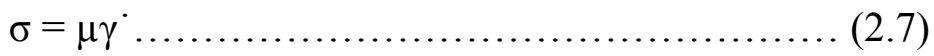

where, $\sigma$ is proportional to the shear rate $\gamma^{\circ}$; and the constant of proportionality, $\mu$, is known as the coefficient of viscosity and results from the lack of slipperiness.

Gases, simple organic liquids, solutions of low molecular weight inorganic salts, molten metals are all Newtonian fluids. Thus, the classification of fluids may be according to their response to an applied shear stress or shear rate, leading to the so called "Newtonian" and "nonNewtonian" fluids. ${ }^{25}$ The next section is focused on discussing the behavior of incompressible fluids under shear stress and shear rate leading to non-Newtonian fluids. A non-Newtonian fluid is one whose flow curve shows an apparent viscosity that depends on flow conditions such as flow geometry, shear rate, angular frequency, etc. (Figure 2.12). 

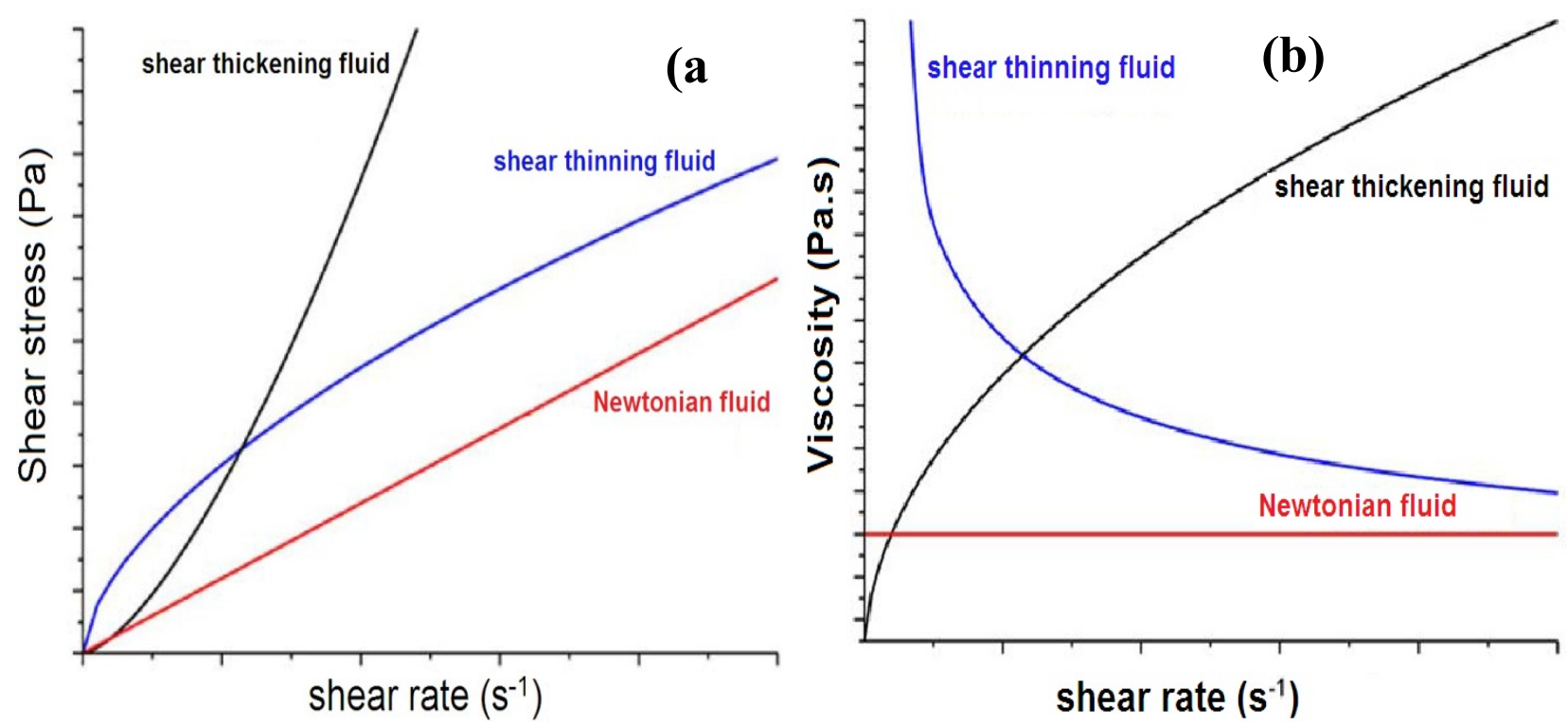

Figure 2.12 Steady state (a) flow behavior (b) viscosity of Newtonian and non-Newtonian fluids

\subsubsection{Viscoelastic Properties of Gels}

Macroscopically, a gel is solid-like but exhibits viscoelastic rheological behavior ${ }^{24-28}$. Hence, viscoelastic properties on intermediate time scales are most appropriate for studying gelation. Small strain tests are preferable, since they avoid rupturing the fragile network structure. ${ }^{26}$ No specific equipment is required beyond what one would use for characterizing viscoelasticity in liquids or solids. Most common are rotational rheometers with concentric disk fixtures (Figure 2.13), cone and plate fixtures, or Couette geometry. 


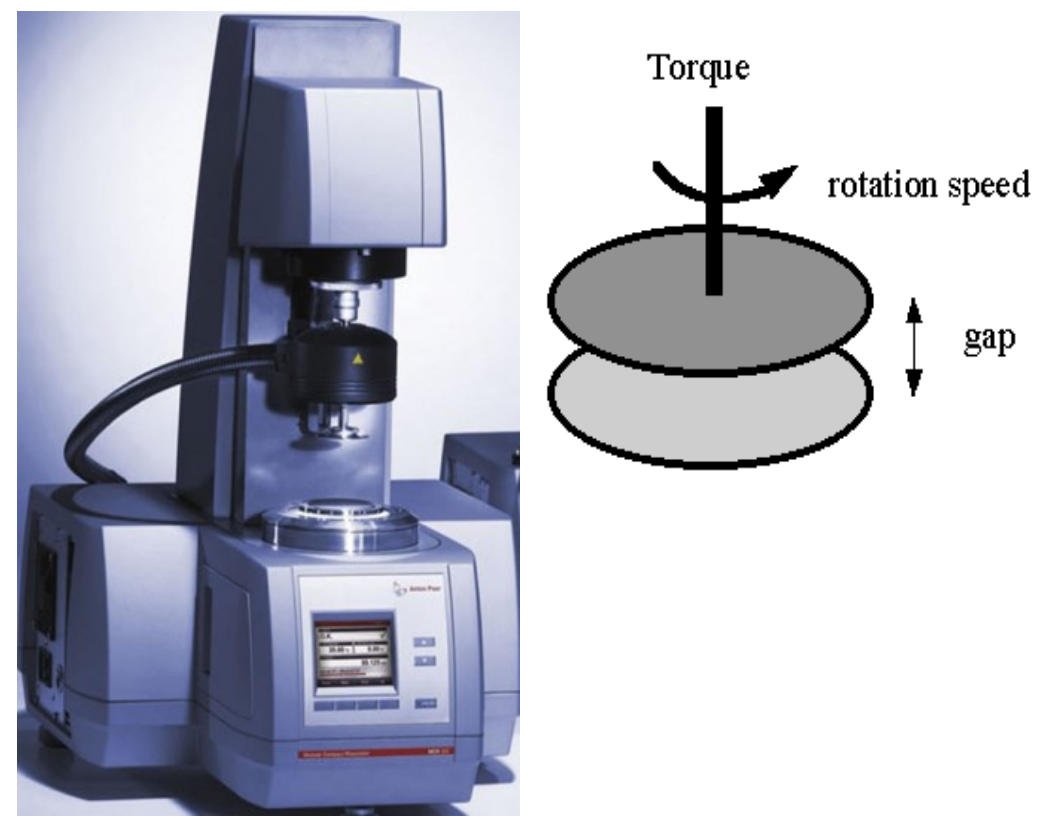

Figure 2.13 AntonPaar MCR 502 Series Rheometer with PP25 Parallel Plates

Typically, commercial rheometers probe frequencies up to tens of Hz. The upper range is limited by the onset of inertial effects, when the oscillatory shear wave decays appreciably before propagating throughout the entire sample. ${ }^{26}$ If the shear strain amplitude is small, the structure is not significantly deformed and the material remains in equilibrium; in this case, the affine deformation of the material controls the measured stress. The time-dependent stress is linearly proportional to the strain, and is given by:

$$
\sigma(t)=\gamma_{0}\left[G^{\prime}(\omega) \sin (\omega t)+G^{\prime \prime}(\omega) \cos (\omega t)\right]
$$

where, $G^{\prime}(\omega)$ is the response in phase with the applied strain and is called the elastic or storage modulus, a measure of the storage of elastic energy by the sample. $G^{\prime \prime}(\omega)$ is the response out of phase with the applied strain, and is called the viscous or loss modulus, a measure of viscous dissipation of energy. The complex shear modulus is defined as:

$$
\mathrm{G}^{*} \equiv \mathrm{G}^{\prime}+\mathrm{iG}^{\prime \prime}
$$


A rheometric measurement normally consists of a strain (deformation) or a stress analysis at a constant frequency (normally $1 \mathrm{~Hz}$ ) combined with a frequency analysis, e.g. between 0.1 and $100 \mathrm{~Hz}$. The strain sweep gives information of the elastic modulus G', the viscous modulus $\mathrm{G}^{\prime \prime}$ and the phase angle $\mathrm{d}$. A large value of $\mathrm{G}^{\prime}$ in comparison of $\mathrm{G}^{\prime \prime}$ indicates pronounced elastic (gel) properties of the product being analyzed. For such a product the phase angle is also small, for example, $20^{\circ}$; since a phase angle of $0^{\circ}$ means a perfectly elastic material and a phase angle of $90^{\circ}$ means a perfectly viscous material. The frequency sweep gives information about the gel strength where a large slope of the G' curve indicates low strength and a small slope indicates high strength.

Oscillatory rheology is a powerful characterization tool because by varying the amplitude and frequency of the applied strain, a wide range of timescales and behaviors can be studied. ${ }^{28}$ For this thesis, we will limit our experiments to small amplitude experiments within the linear viscoelastic regime which allows an investigation of the gel response without disruption of the gel structure.

We used a Anton Paar MCR 502 Rheometer, with parallel plates PP25 and Rheoplus/32 V3.61 application. The edges of the plate were sealed with wax prior to the experiments. Rheological experiments were carried out at the Centre de Recherche en Plasturgie et Composites, École Polytechnique de Montréal, Montréal Canada. 


\subsection{References}

1. Skoog, D.A.; Leary, J.J. Principles of Instrumental Analysis, 4th Ed., Saunders College Publishing: New York, 1992, pp. 252-280.

2. Kalsi, P.S. Spectroscopy of Organic Chemistry, New Age International Ltd, 4th Ed., 2000, pp. 60-63.

3. Pavia, D.L.; Lampman, G.M.; Kriz, G.S. Introduction to Spectroscopy, Harcourt College Publisher, 2001, 3rd Ed., pp. 41.

4. Osland, R.J. Principles and Practices of Infrared Spectroscopy, PyeUnicam Ltd.: Great Britain, 1985, pp. 5-32\&64-69.

5. Smith, B. Infrared Spectral Interpretation, CRC Press, 1999.

6. Theophanides, T. Infrared Spectroscopy - Materials Science, Engineering and Technology, InTech: New York, 2012.

7. Bower, D. I. The Vibrational Spectroscopy of Polymers, Cambridge University Press: New York, 1992.

8. Bushong, S.C. Magnetic Resonance Imaging, 2nd Ed., Mosby-Year Book, Inc., 1996.

9. (a) Silverstein, R. M.; Bassler, G. C; and Morrill, T. C. Spectrometric Identification of Organic Compounds, 5th Ed., Wiley, 1991.(b) Nyquist, R.A. Interpreting Infrared, Raman, and Nuclear Magnetic Resonance Spectra, Academic Press: New York, 2001, pp. 900.

10. Wendlandt, W.W. Thermal Analysis, Wiley Interscience: New York, 1986, pp. 667.

11. (a) Fox, T.G.; Flory, P.J. J. Appl. Phys. 1950, 21, 581. (b) Fox, T.G.; Flory, P.J. J. Polym. Sci.1954, 14, 315.

12. Chapiro, A. Radiation Chemistry of Polymeric Systems, Interscience: London, 1962. 
13. Klug, H.P; and Alexander, L.E.X-Ray Diffraction Procedures: For Polycrystalline and Amorphous Materials $2^{\text {nd }}$ Edition, John Wiley: New York, 1974.

14. Mertz, J. Introduction to Optical Microscopy, Roberts and Company Publishers: Colorado, 2010.

15. Pawley, J. Handbook of Biological Confocal Microscopy, 3rd Ed.;Springer Science+Business Media, LLC. New York, 2006; Chap. 1.

16. Williams, B.; Carter, C. Transmission Electron Microscopy: A Textbook for Materials Science, Plenum Press: New York, 1996; Chap. 1 and 2.

17. Saville, B. P. in Applied Polymer Light Microscopy; Hemsley, D. A., Ed.; Elsevier Science Publishers Ltd.: New York, 1989; Chap. 4.

18. Kalantar-zadeh, K.; and Fry, B. Nanotechnology-Enabled Sensor, 1st edition; Springer Science: New York, 2008.

19. Gore, M.G. Spectrophotometry and Spectrofluorimetry: A Practical Approach, Oxford Univ. Press.2000.

20. Rao, C.N.R. Ultra-Violet and Visible Spectroscopy: Chemical Applications, 3th Ed. Butterworths: London, 1975, pp. 100.

21. Pesce, A.M.; Rosen, C.-G.; Pasby, T.L. Fluorescence Spectroscopy, Marcel Dekker Inc: New York, 1971

22. Eichinger, B.E.; Khare, R. Molecular Modeling in: Encyclopedia of Polymer Science and Technology, Interscience: New York, 2003.

23. HyperChem Manual, Hypercube Inc., Waterloo, 2002

24. Ferry, J. Viscoelastic properties of polymers, Wiley: New York, 1980. 
25. Grillet, A; Wyatt, N.B.; and Gloe, L. M. Polymer Gel Rheology and Adhesion, Rheology, Dr. Juan De Vicente (Ed.), InTech.: New York, 2012. Chap. 3.

26. Macosko, C. W. Rheology: Principles, Measurements, and Applications, Wiley-VCH, Inc.: New York, 1994.

27. Patel, S. K.; Malone, S.; Cohen, C.; Gillmor, J. R. \& Colby, R. H. Elastic Modulus and Equilibrium Swelling of Poly(dimethylsiloxane) Networks. Macromolecules 1992, $25,5241-5251$.

28. Gittes, F.; Schnurr, B.; Olmsted, P.D.; MacKintosh, FC.; Schmidt, C.F. Microscopic viscoelasticity: shear moduli of soft materials determined from thermal fluctuations. Physical Review Letters 1997, 79, 3286-3289. 


\section{CHAPTER 3}

\section{Thermo-Reversible Physical Gels of Poly (Dimethylsiloxane) without Crosslinks or Functionalization}

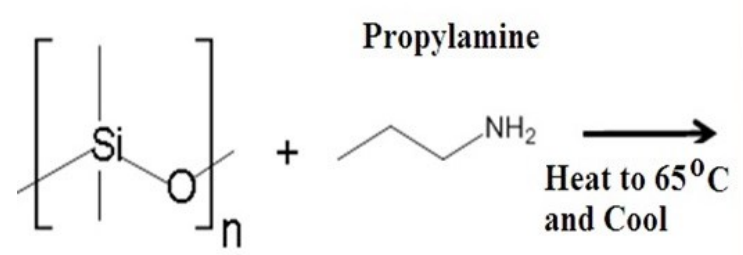

PDMS

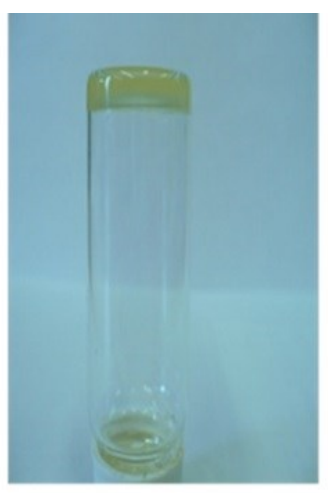

Thermo-reversible Physical Gel

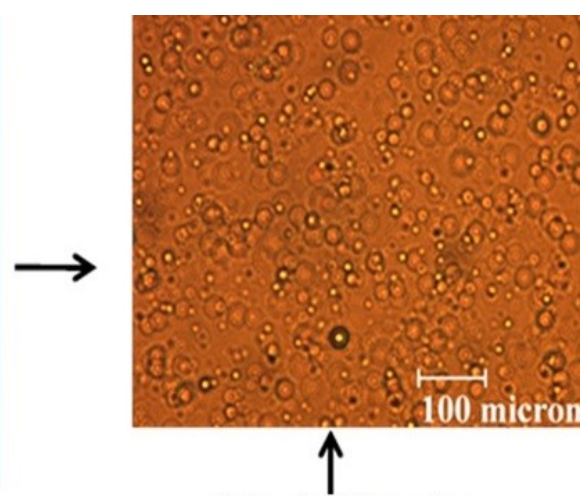

Spherical Domains with Network

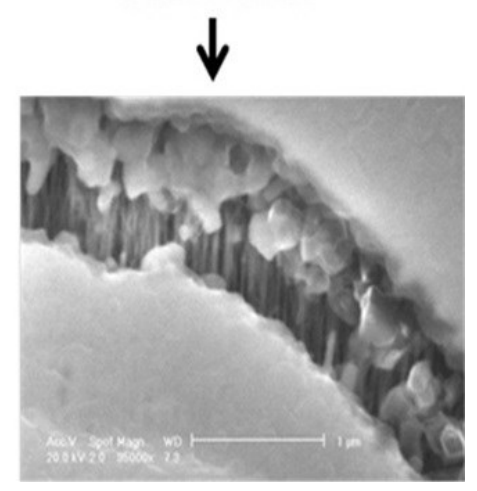




\subsection{Introduction}

Gels can generally be classified as non-reversible, when made with covalently formed cross links (chemical gel) and reversible, if non-covalent interactions such as hydrogen bond, ionic bond or solvophobic interactions are used (physical gel) ${ }^{1,2}$. Gelation of the latter type in polymers is considered to be the result of liquid-liquid phase separation and vitrification ${ }^{3}$. Such a phenomenon could also be accompanied by crystallization in the case of semicrystalline polymers $^{4-12}$. This type of gel is usually made by dissolving the gelator in a solvent by heating, and then cooling to a lower temperature for gel formation ${ }^{3}$.

Poly (dimethylsiloxane) (PDMS) is the most commonly used silicon-based organic polymer with diverse applications ${ }^{13-16}$ as e.g., a sealant, separation layer for volatile organic components etc. ${ }^{17}$. It possesses properties such as high hydrophobicity, permeability, and good thermal and mechanical resistance which make it an interesting membrane material for organic extraction and is easy to manufacture ${ }^{18}$. Membrane transport properties depend on the microstructure of the polymer network and therefore on its preparation conditions. PDMS can be cross-linked into networks ${ }^{13-16}$.

Gels of PDMS have so far been obtained only via the "chemical gel" route, by cross linking and swelling with a solvent. Typically this is obtained with PDMS networks formed with the base polymer and a curing agent, and cross-linked through a hydrosilylation reaction under thermal curing with an organo-metalic catalyst such as platinum ${ }^{19-21}$. There have been several studies in this regard ${ }^{22-26}$ and gels containing fillers have also been reported $^{27-29}$. Suzuki et al ${ }^{30}$ reported the gelation of low molecular weight PDMS functionalized with a known gelating agent, with the latter directing the gelation. These authors used bis(3-aminopropyl)poly(dimethylsiloxane) $\left(\mathrm{M}_{\mathrm{W}}=900-1000\right)$ and poly[dimethylsiloxane-co-(3-aminopropyl) 
methylsiloxane], functionalized with a L-lysine derivative, the latter being the gelating agent. There has been no report to date on gels of PDMS via the physical gelation route, without the use of catalysts and cross links or without functionalization. (When we refer to "PDMS gel" in this chapter, we denote the physical gels in which PDMS is the gelator of an organic solvent).

Since we studied the gelation of perylene diimide substituted with PDMS (Chapter 4), we examined the possibility of obtaining gels of PDMS without cross links or functionalization, and succeeded. In this chapter, we describe the thermo-reversible gelation of PDMS via the physical route, without any functionalization. The choice of effective solvent for the preparation of polymer gels or organogels of small molecules has so far been discovered by serendipity than by a priori design ${ }^{31}$. We discuss a solvent system, propylamine or hexylamine, gelled by PDMS, without any filler, catalysts or chemical crosslinks.

\subsection{Experimental}

Poly (dimethylsiloxane) $\left(\mathrm{M}_{\mathrm{w}}: 182,600 ; \quad \mathrm{M}_{\mathrm{n}}: 106,000\right.$, CASRN 9016-00-6), propylamine and hexylamine were purchased from Aldrich Chemical Company. A low molecular weight PDMS (silicon putty, Mw: 323) was purchased from General Electric Company (Batch 613-01613 GE \# SE-30).

Clear solutions of PDMS in propylamine or hexylamine were prepared with constant stirring at temperatures ranging from $65-90^{\circ} \mathrm{C}$. As in our previous studies ${ }^{32,33}$, gels were then prepared by quenching the solution in dry ice or by slow cooling to room temperature by turning off the hot plate. Concentrations of PDMS ranging from $0.03-0.30 \mathrm{M}(5.478 \mathrm{~g} / \mathrm{L}$ to $18.26 \mathrm{~g} / \mathrm{L})$ were used. Gels were prepared in closed vials to avoid the evaporation of the solvent. Gelation was tested by tube inversion (with no flowing solvent). 


\subsubsection{Results and Discussion}

Gels with crystallizable polymers such as isotactic polystyrene, poly (vinyl chloride), and polyethylene have been studied $^{5-12}$, and liquid-liquid phase separation accompanied by crystallization has been attributed to network formation. The gel of atactic polystyrene, which is a non-crystallizable polymer has also been found to form networks, or spherical domains connected by networks ${ }^{3,34}$. In the present case, PDMS is crystallizable below $-40{ }^{\circ} \mathrm{C}$. The glass transition $\left(\mathrm{T}_{\mathrm{g}}\right)$ itself is below this temperature at $-125^{\circ} \mathrm{C}$, as compared to the $\mathrm{T}_{\mathrm{g}}$ of about $100^{\circ} \mathrm{C}$ for atactic polystyrene.

As we mentioned above, gels of PDMS have never been prepared without crosslinks, and these constitute irreversible gels. Solvents for thermo-reversible physical gels have so far been discovered by serendipity than by a priori design ${ }^{31}$. There have been recent studies ${ }^{35-37}$ aimed at developing predictability of gelation, based on the solubility parameters of solvents and organo gelators, solvent viscosity, solid-liquid phase boundary of the gelator etc.. However, in a recent review, Das et al ${ }^{38}$ acknowledged the hurdles "that still limit the understanding of the intricacies of gel formation through the process of self assembly". While these studies relate to organogels involving small molecule gelators, there is also a distinction between the formation of organo and polymer based gels. With the former, network formation and solvent trapping depends on self assembly of the small molecule gelator via by non-covalent interactions such as hydrogen bond. However, in the case of polymer gelators, chain entanglement and network formation trap the solvent causing gelation, as in the case of atactic polystyrene ${ }^{34}$ and atactic PMMA ${ }^{39}$. Forces such as hydrogen bonding are not a requirement.

Whitesides et $\mathrm{al}^{40}$ examined the relationship between the solubility parameters of a number of solvents and the swellability of cross-linked PDMS and found diisopropylamine to 
cause the most swelling, followed by triethylamine and pentane. If a solvent swells the polymer at room temperature, chain expansion would occur. If the polymer then dissolves upon increasing the temperature, entanglement of the coils could follow upon lowering the temperature which would trap the solvent and cause gelation. Based on this premise, we experimented with these solvents. However, all three of the above solvents dissolved our noncross-linked polymer at room temperature and hence were not suitable for the gelation experiments. The list of solvents and their behavior are given in Table 3.1.

Table 3.1. The Effect of Various Solvents on the Poly (dimethyl siloxane) Sample

\begin{tabular}{lcl}
\hline Solvent & Swelling at room temperature? (visual) & Result after heating and cooling \\
\hline Hexyl amine & Yes & Gel \\
\hline & Yes & Clear Solution \\
\hline Triethyl amine & Yes & Turbid Solution/Precipitate \\
\hline Hexane & No & Precipitate \\
\hline Acetonitrile & & Turbid Solution \\
\hline
\end{tabular}

* The images of the experimental vials are shown in Figure 3.1

This led us to choose linear amines, viz., propylamine and hexylamine. Here we show that thermo-reversible physical gels with PDMS can be prepared without crosslinks or functionalization, with these solvents. We will discuss mainly the features of the gels prepared with propylamine in this paper. 


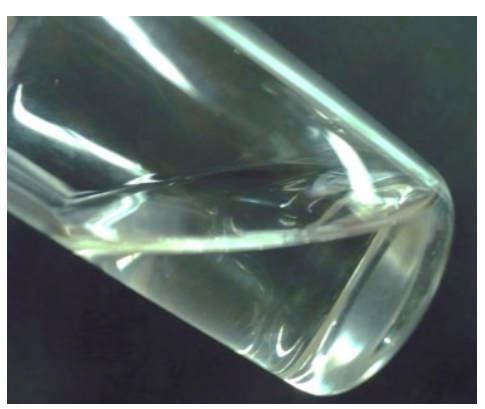

Diisopropylamine

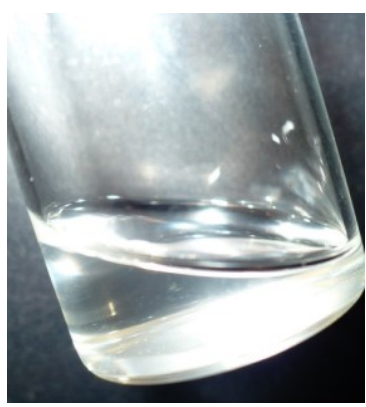

Triethylamine

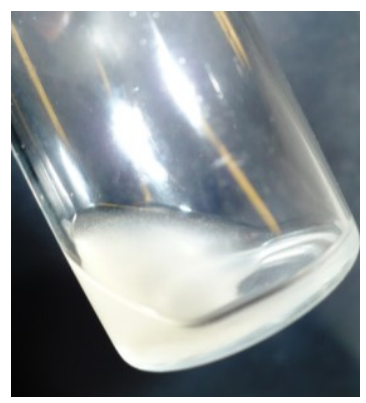

Pentane

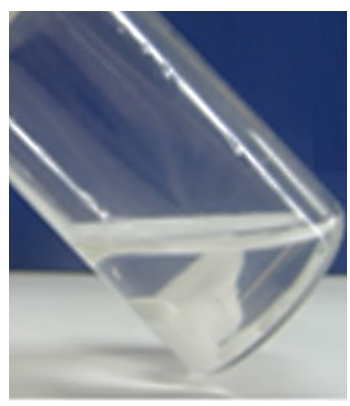

Ethyl acetate

Figure 3.1 Images of PDMS in Various Solvents

Clear solutions were obtained above $70{ }^{\circ} \mathrm{C}$ with propylamine and the onset of gelation occurred at $65{ }^{\circ} \mathrm{C}$. Complete immobilization of the solvent was achieved at $38-40{ }^{\circ} \mathrm{C}$. With hexylamine, a temperature of $80{ }^{\circ} \mathrm{C}$ was required for obtaining a solution and gelation was complete at $30-35{ }^{\circ} \mathrm{C}$. Upon heating the PDMS/propylamine solution to $70{ }^{\circ} \mathrm{C}$, the polymer dissolves, as mentioned above and the chain expands. The temperature coefficient of the end-toend distance ${ }^{41}$ of PDMS is also positive $\left(0.78 \mathrm{deg}^{-1}\right)$ and much higher than e.g., that of polystyrene $\left(0.3 \mathrm{deg}^{-1}\right)$. Upon reducing the temperature, the chains collapse, entangling themselves in a network, trapping the solvent and cause gelation. The minimum concentration required for gelation was $0.03 \mathrm{M}$ with both solvents. Figure 3.2 shows the inverted vials containing PDMS and the gel prepared with $0.08 \mathrm{M}$ concentration of PDMS in propylamine. While the original sample of PDMS in the inverted vial flows along the walls, the gel prepared as above does not. It remained so for several weeks. All gels were opaque when cooled; these gels were dried at room temperature for a few days to prepare xerogels.

As seen in Figure 3.3, the xerogels retain their shape after solvent evaporation, and do not collapse to a film. The entanglements that occur during the cooling of the solution (and gelation) serve as physical cross-links (as discussed below) and persist in the xerogels (as seen from the G' and G" described later). 


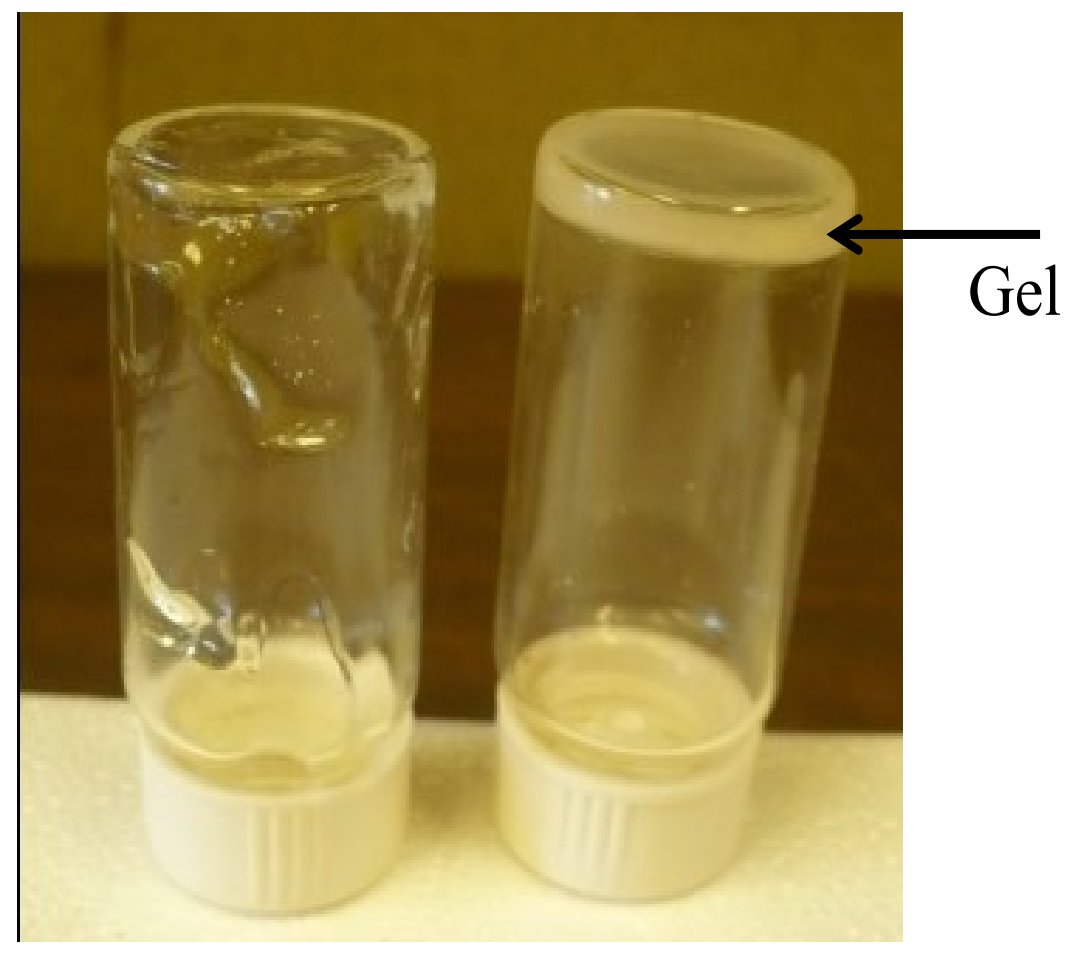

Figure 3.2 PDMS gel (at right) prepared with propylamine as the solvent. The flow of PDMS starting material is seen in the vial on left.

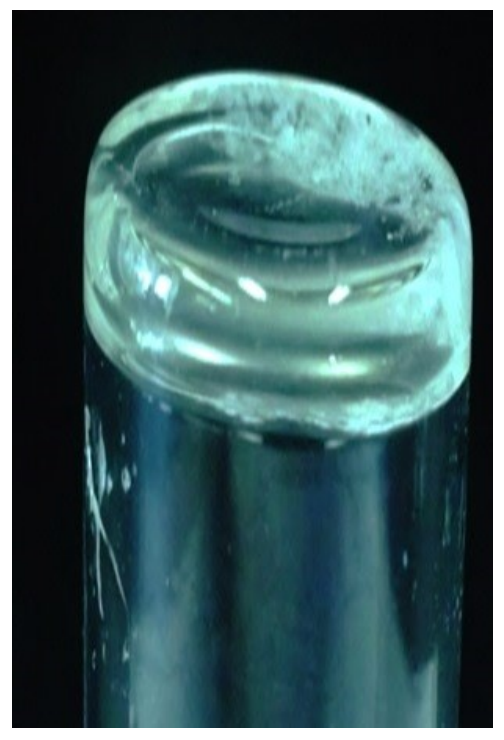

PDMS Gel

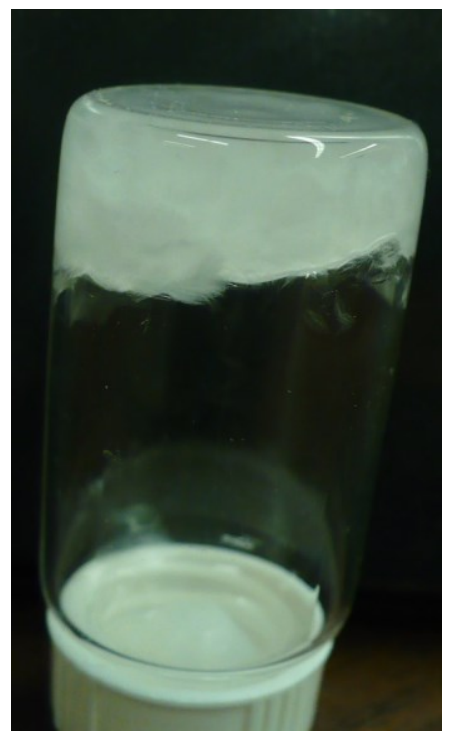

PDMS Xerogel

Figure 3.3 Images of inverted vials containing PDMS gel and xerogel 
No specific molecular interaction between PDMS and propylamine is indicated by NMR spectra. NMR spectra of the bulk sample and the gels were similar to those reported in the literature for PDMS. ${ }^{42}$ Figure 3.4 shows the ${ }^{1} \mathrm{H}$ NMR spectra of the PDMS gel and the bulk PDMS in the siloxane region, which are very similar.

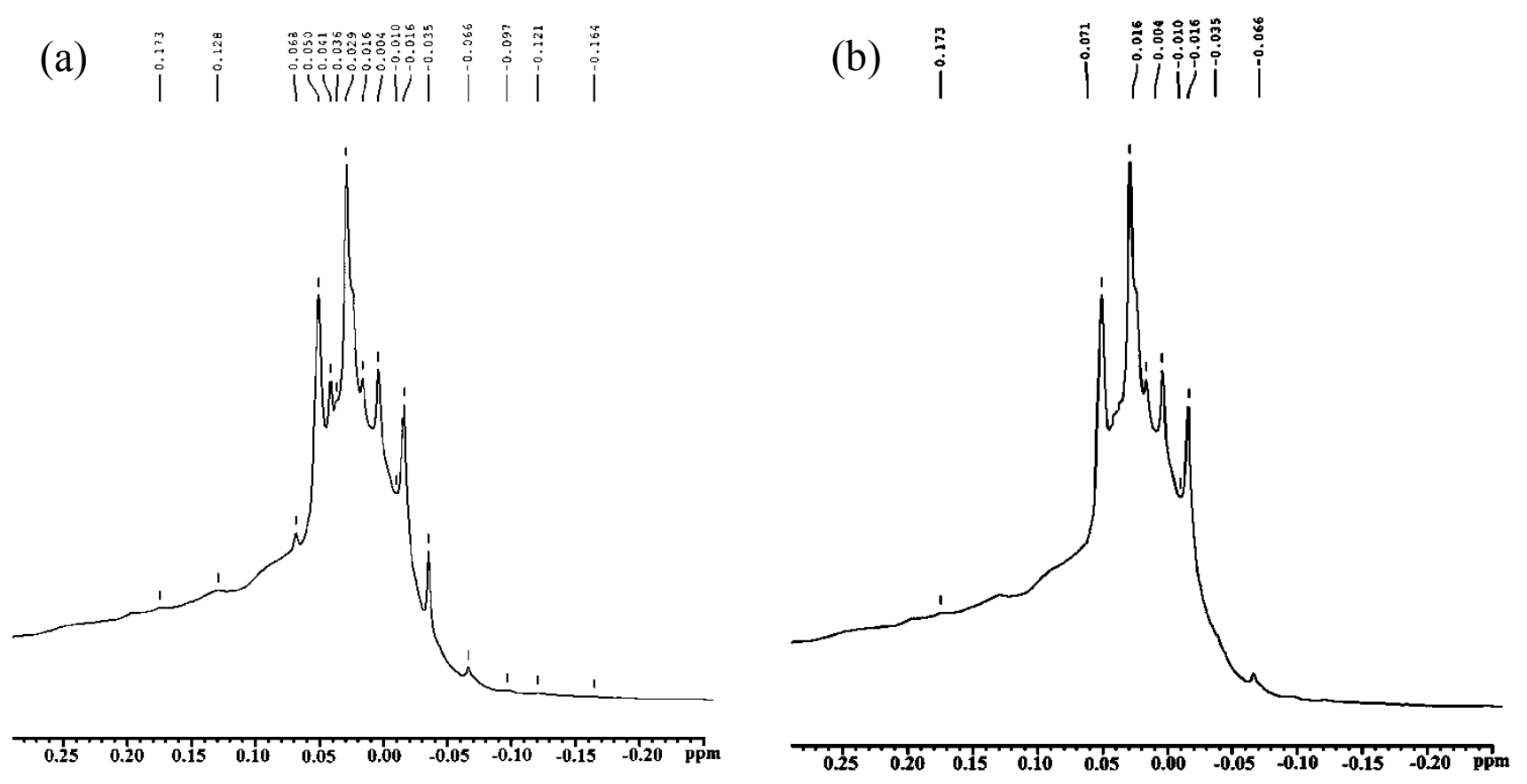

Figure 3.4 NMR spectra of (a) PDMS/ propylamine gel (b) bulk PDMS in the siloxane region

However, the FTIR spectra (Figure 3.5) show broadening of the gel spectrum in the Si-OSi absorption region, compared to those of the bulk PDMS, xerogel and heptane solution. Mark ${ }^{43}$ determined a dipole moment of $\mu=0.60 \mathrm{D}$ for the Si-O and O-Si bonds in PDMS. The $\mu$ of propylamine is $1.4 \mathrm{D}$. It is possible that dipole interactions arise in the solution and the gel. 


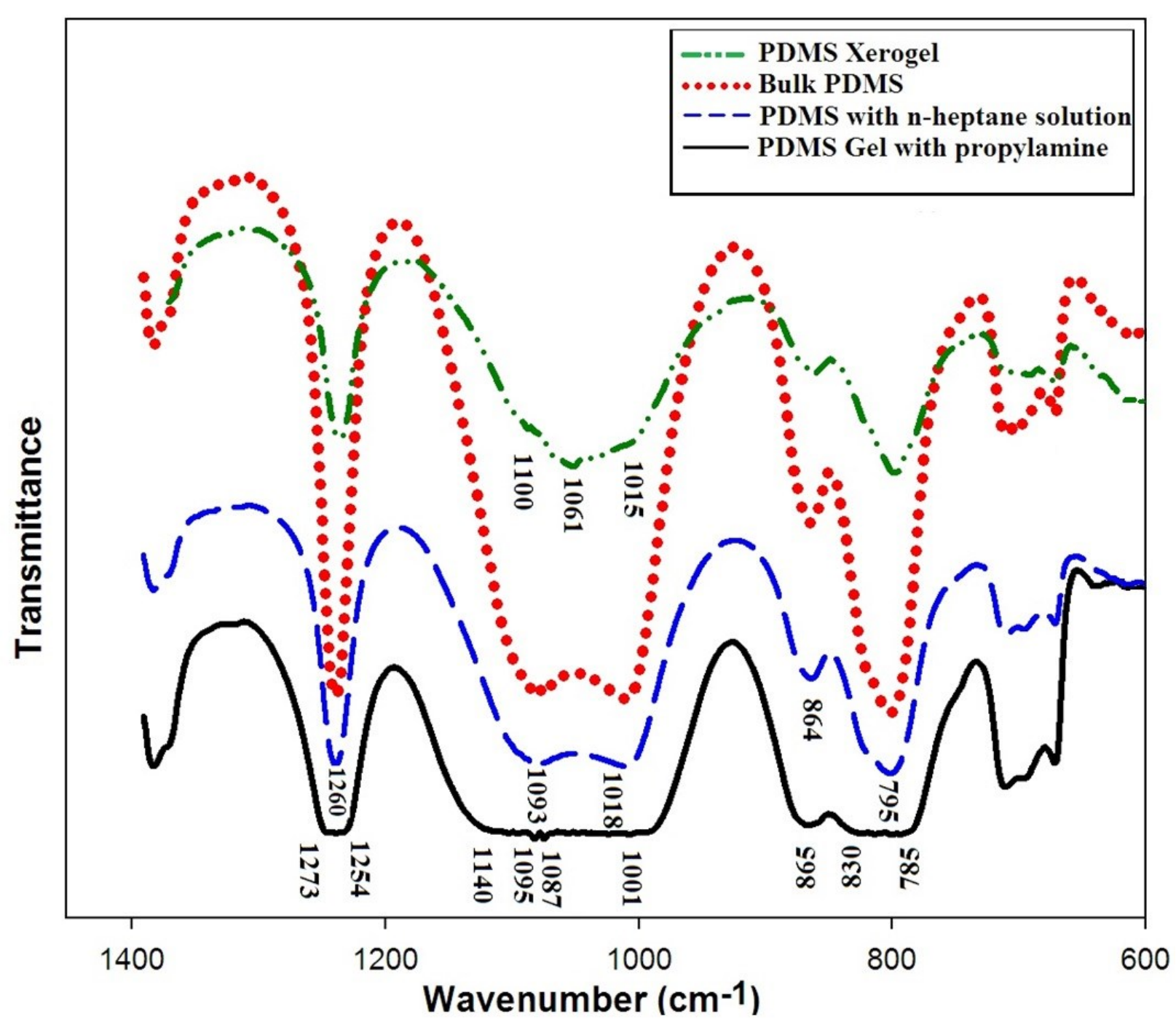

Figure 3.5 FTIR spectra of PDMS/propylamine gel, xerogel, PDMS/hexane solution and bulk PDMS

In contrast to atactic polystyrene mentioned above, PDMS is a 'gummy' polymer. It flows at room temperature and it gels the above low molecular weight organic solvents. However, this is not a case of gels of emulsions. The emulsions require surfactants for stabilization and cross-linkers for gelation. ${ }^{44-47}$ In the present case, neither surfactants nor crosslinkers are used. 


\subsubsection{Morphology of High Molecular Weight PDMS}

Due to the low $\mathrm{T}_{\mathrm{g}}$ of PDMS $\left(-125^{\circ} \mathrm{C}\right)$ we used optical microscopy mainly, and the ESEM and TEM for a few experiments. The OM of the slow-cooled gel and the xerogel at room temperature (using propylamine) are shown in Figure 3.6a and 3.6b. It is seen that the morphology consists of spherical domains, with a broad distribution of sizes, ranging from 6 to $25 \mu \mathrm{m}$, with an average of $14 \mu \mathrm{m}$. The size distribution is shown in Figure S5a. Such spherical structures have been seen in the case of filler-loaded and cross linked gels of PDMS ${ }^{22}$. The higher magnification images (Figure 3.6d) obtained with the E-SEM show even much smaller domains of about $300 \mathrm{~nm}$. A three-dimensional image constructed by recording several layers with the confocal laser scanning microscope is shown in Figure 3.6c. The TEM image of the xerogel in Figure 3.6e shows even much smaller spherical domains, and fibrils connecting these domains. The larger spherical domains observed in the optical micrographs and the SEM could be the aggregated structures of smaller spheres seen in the TEM image. Maintaining the sample at the gelation temperature for 30 minutes led to uniform spherical domains, as seen in Figure 3.6f and S5b (see DSC results below). Formation of spherical domains with network connectivity (beaded string structure) has been seen with the gelation of atactic polystyrene and was discussed in detail by Keller ${ }^{34}$. This was attributed to phase segregation via nucleation. However, nucleation here is not due to crystallization as in the case of isotactic or syndiotactic polystyrenes or polyethylene. It is due to the segregation (bunching up) of the chains as shown schematically in Figure 3. PDMS being a highly flexible polymer with a $T_{g}$ far less than that of polystyrene, spherical rather than fibrous aggregation of the chains results during the sol-gel transition. It is known $n^{41,48,49}$ that due to the large difference in the bond angles at O-Si-O $\left(110^{\circ}\right)$ and $\mathrm{Si}-\mathrm{O}-\mathrm{Si}\left(145^{\circ}\right)$ along the chain, the trans-trans conformation of the contiguous bonds would 
lead to chain closure after about eleven units. In the crystalline state ${ }^{50}$ (at $-90{ }^{\circ} \mathrm{C}$ ), the PDMS chain adopts a flat helical conformation, with a displacement of about $35^{\circ}$ from the trans conformation. Further, because of the long Si-O and Si-C bonds (1.64 and $1.90 \AA$, respectively), the energy difference between the trans and the gauche conformations is only of the order of $\mathrm{RT}^{41,48}$. Such a lack of preference for a predominant conformation and low barrier between the trans and gauche states can be expected to lead to coiled segments, resembling macrocyclization ${ }^{49}$, which would facilitate entanglement of the coils during gelation.

As the gelation proceeds from solution, the chains entangle to form spherical domains and these domains are connected by parts of the chains to form a network. This is seen in the TEM image in Figure 3.6e and the E-SEM image of a fracture domain (Figure 3.7b), recorded at $-140{ }^{\circ} \mathrm{C}$. The thickness of the fibrils seen in the fracture in the E-SEM image is about $40 \mathrm{~nm}$, indicating that these are bundles of a few chains. The inset in Figure 3.6d also shows fibrils connecting the spherical domains. The fibrils in the TEM image are much thinner. Such entangled domain formation is facilitated by the molecular flexibility of the chain as discussed above. The TEM image (Figure 3.6e) shows spherical domains of $10-20 \mathrm{~nm}$ in diameter. The root-mean-square unperturbed end-to-end distance measured by Flory et $\mathrm{al}^{51}$ for a high molecular weight PDMS is $73 \mathrm{~nm}$. In a good solvent condition, it can be expected to be longer. Thus a chain could bridge these spherical domains. The root-mean-square unperturbed radius of gyration reported $^{52}$ from small angle neutron scattering experiments is $4 \mathrm{~nm}$. Jadav et $\mathrm{al}^{53}$ reported $\mathrm{R}_{\mathrm{g}}$ values ranging from 6 to $13 \mathrm{~nm}$ for PDMS samples used for membranes. The $\mathrm{R}_{\mathrm{g}}$ of the spherical domains (Figure 3.6e) with diameters of $10-20 \mathrm{~nm}$ is $3.9-7.7 \mathrm{~nm}$. Thus the $\mathrm{R}_{\mathrm{g}}$ of the PDMS as deduced from the neutron scattering experiments is slightly larger than the $\mathrm{R}_{\mathrm{g}}$ of the spherical domains seen in Figure 3.6e. 

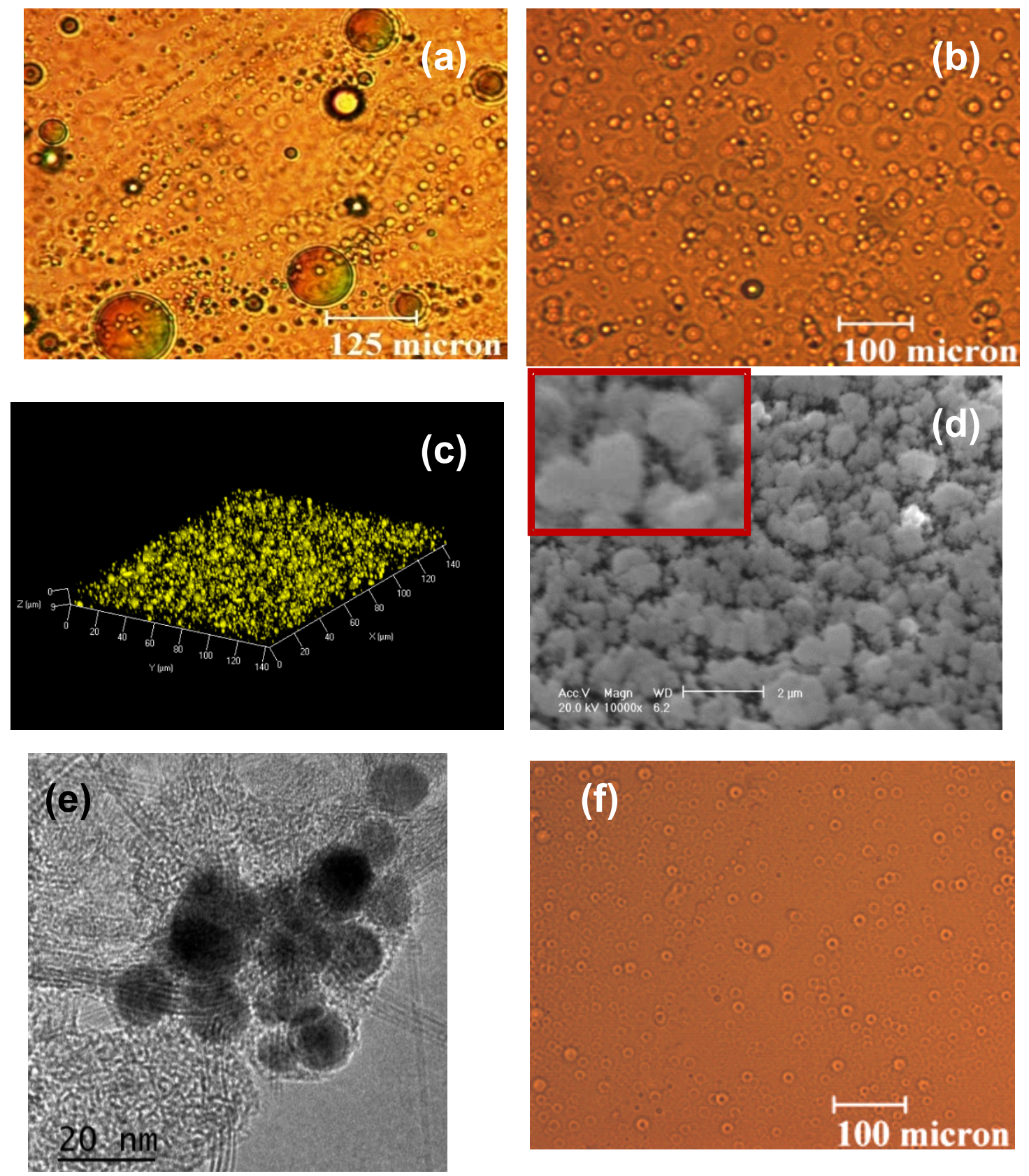

Figure 3.6 (a) Optical micrograph of PDMS gel from propylamine. (b) OM of the xerogel (c) Three dimensional image using confocal laser microscope (d) E-SEM image recorded at $-140{ }^{\circ} \mathrm{C}$; (e) TEM micrograph of the xerogel; (f) Sample kept at gelation temperature for 30 minutes and cooled 


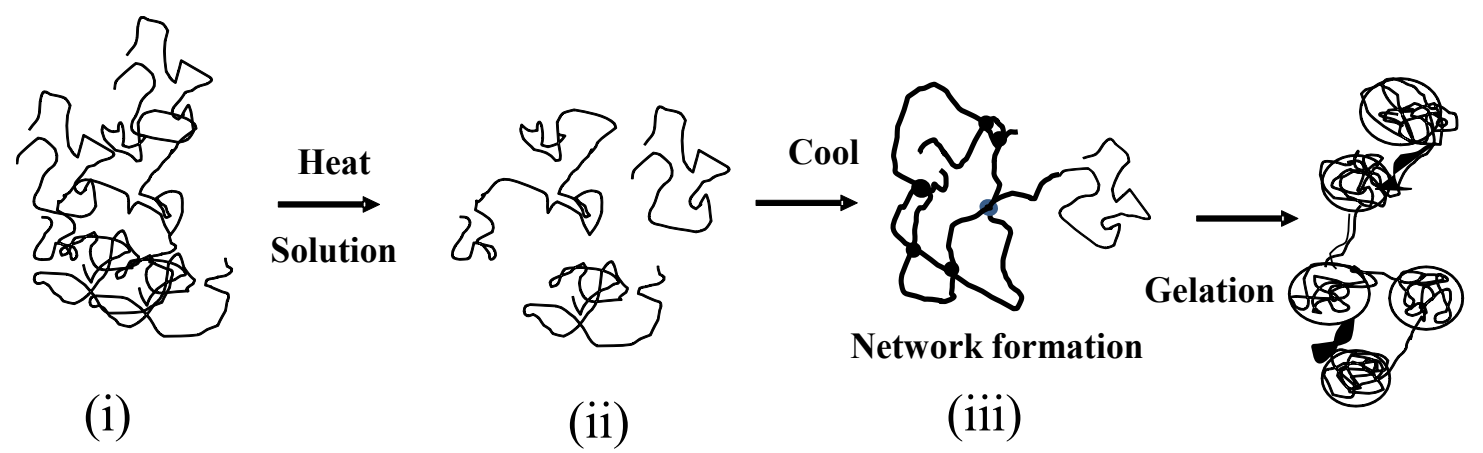

(a)

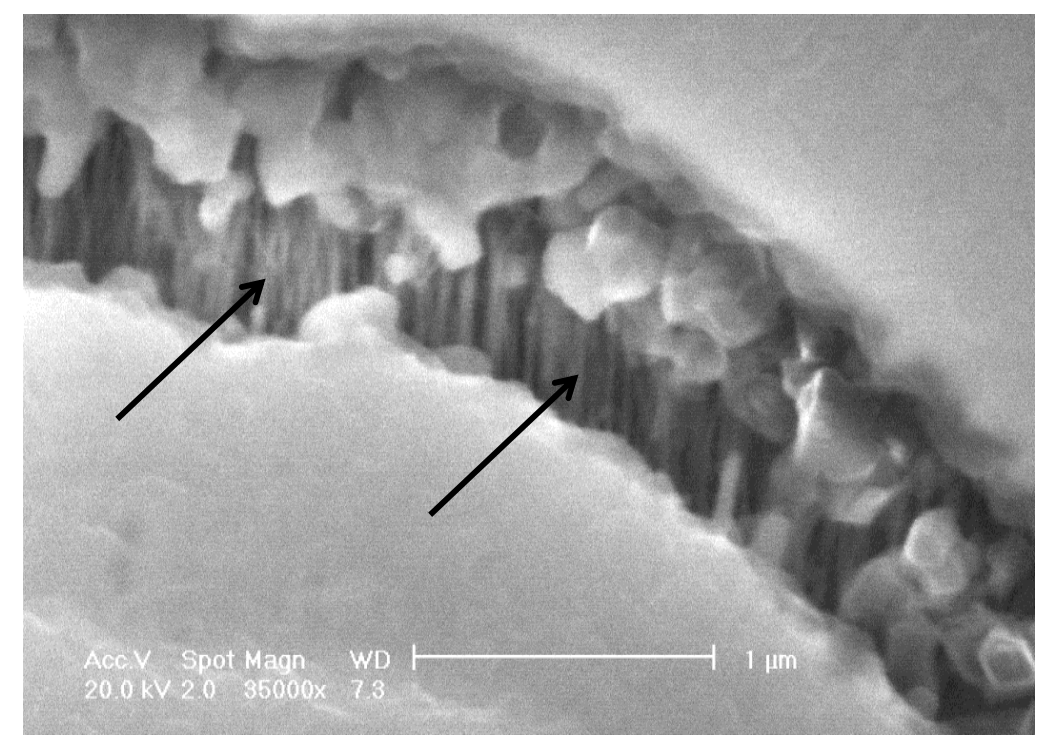

(b)

Figure 3.7 (a) Illustration of the formation of network and spherical domains during gelation with PDMS: (i) chains in bulk; (ii) chains dissolve and expand on heating; (iii) upon cooling, chains start collapsing and forming network trapping the solvent leading to gelation; (b) E-SEM image of a fracture domain showing the fibrils (shown by arrows) between domains

X-ray diffraction showed that the gel as well as the xerogel, do not develop any crystallinity, as seen from Figure 3.8. A diffuse peak with a d-spacing of $6.5 \AA$ is seen, which is typical of diffraction from amorphous polymers. 

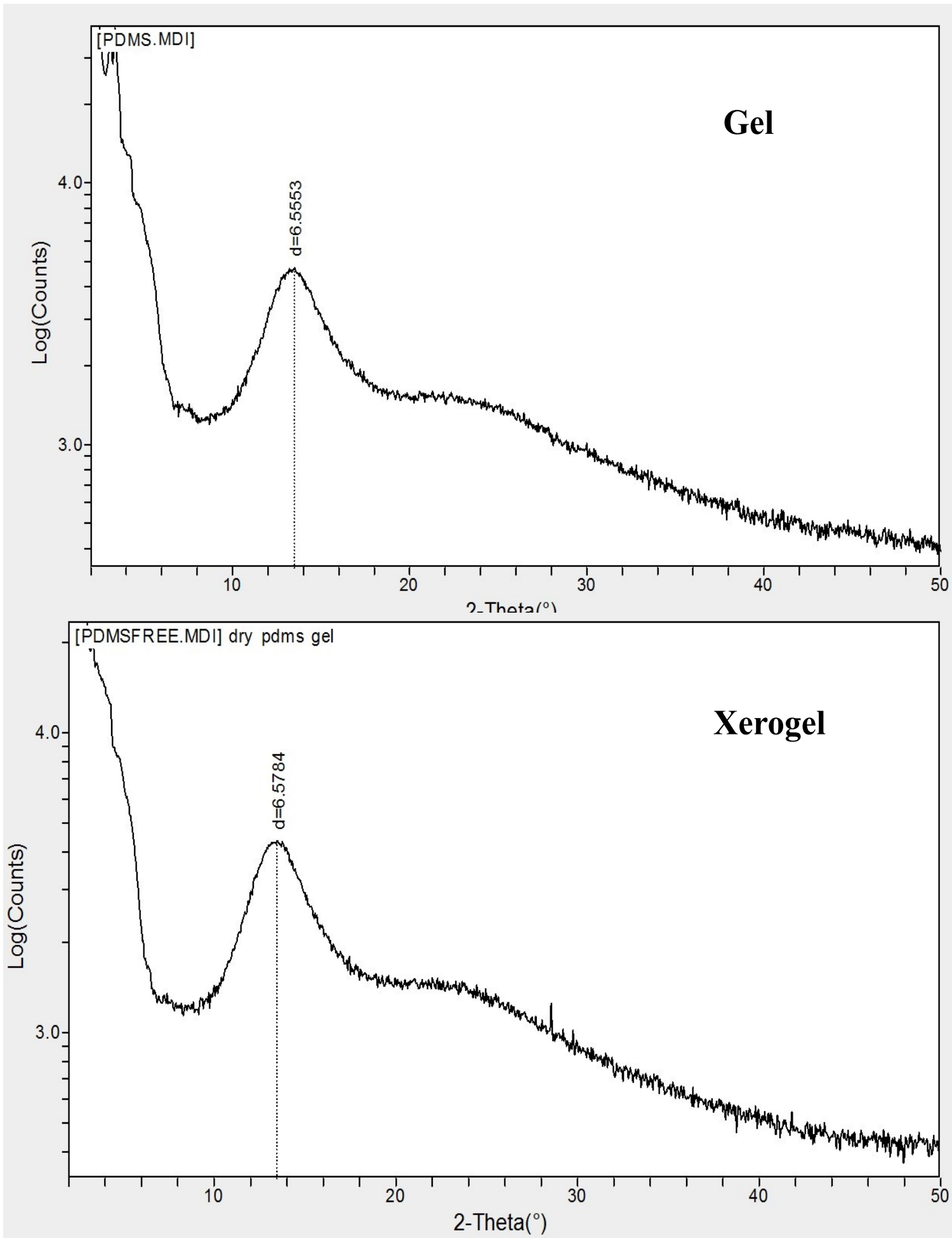

Figure 3.8 X-ray diffraction traces of PDMS gel (top) and xerogel (bottom) 


\subsubsection{Thermal Analysis and Thermo-reversibility}

The thermo-reversibility of the gels was verified with both DSC and OM. The DSC analysis (Figure 3.9) of the gels showed an endotherm at $65^{\circ} \mathrm{C}$ in the first heating cycle corresponding to the gel-sol transition, and a sol-gel transition exotherm at $45^{\circ} \mathrm{C}$ upon cooling. This shows that the gelation is thermo-reversible. With the scan rate of $10{ }^{\circ} \mathrm{C} / \mathrm{min}$ used here, the sample does not have sufficient time to gel during the cooling cycle and a shallow endotherm for gel-sol transition resulted during the second heating cycle. We found that during the cooling cycle, it is necessary to maintain the temperature in the DSC close to the sol-gel transition for about 30-45 minutes to observe the well-defined endotherm in the second heating cycle. Using this approach, an endotherm comparable to that in the first heating cycle was obtained in the second heating cycle also, as seen in Figure 3.9. We noted above that maintaining the sample at the gelation temperature for 30 minutes led to more uniform spherical domains (Figure 3.6e). Repeated heating and cooling in the closed vial (as in Figure 3.1) also confirmed the thermoreversibility of gelation. The gel-sol-gel transitions were observed under the OM as well. For this purpose, a sample of the gel was kept in a cylindrical well attached to the microscope slide, closed with a cover glass, and the temperature was varied with the hot stage. Figure 3.10 shows the micrographs at five different temperatures. Most of the spherical domains became diffuse at $65^{\circ} \mathrm{C}$, and upon cooling, they began reforming at $45^{\circ} \mathrm{C}$, confirming thermo-reversibility. 


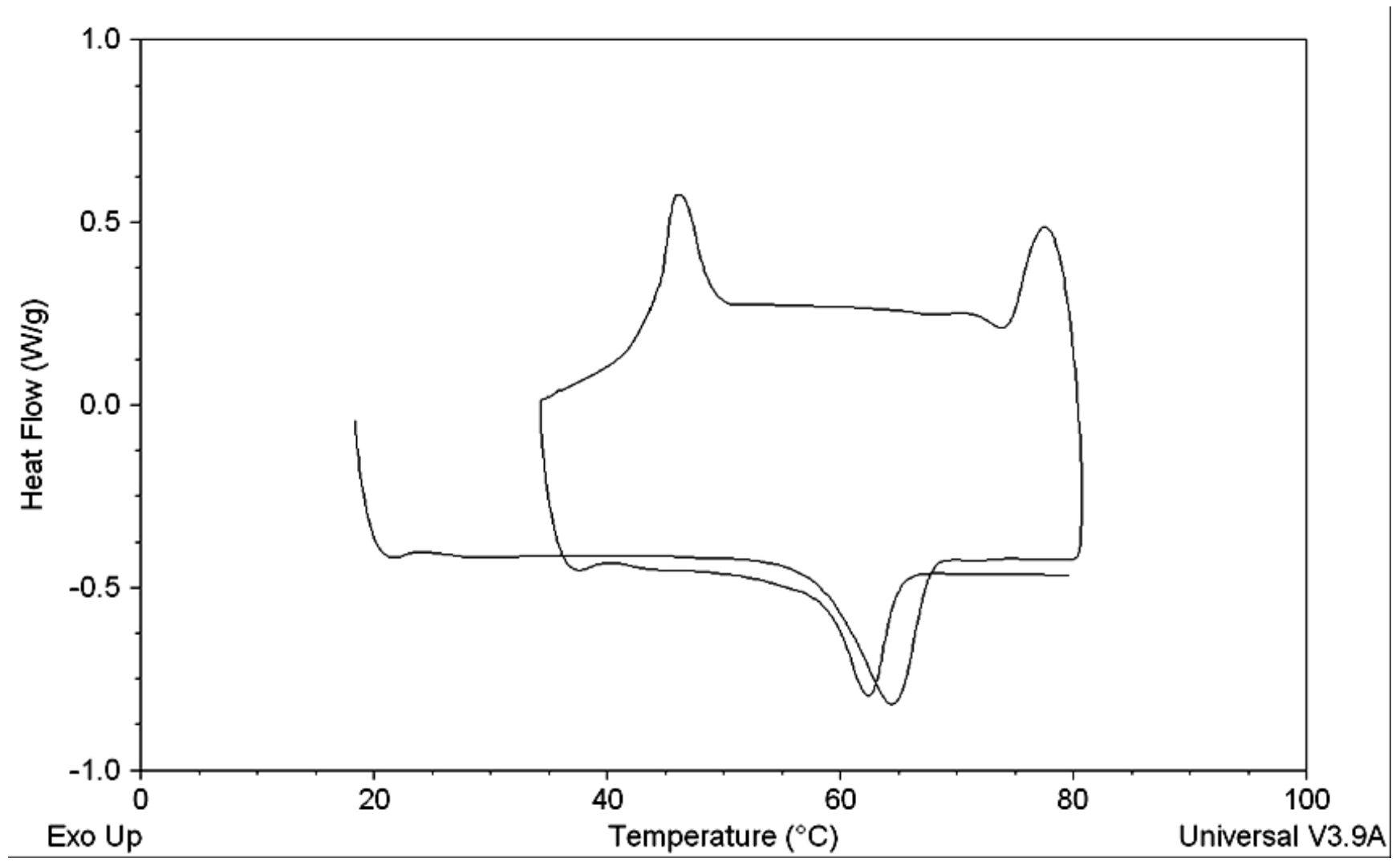

Figure 3.9 DSC traces of gels of PDMS during the heating, cooling and second heating cycles. The sample was kept for 45 minutes at $45^{\circ} \mathrm{C}$ during the cooling cycle to enhance the gel formation 

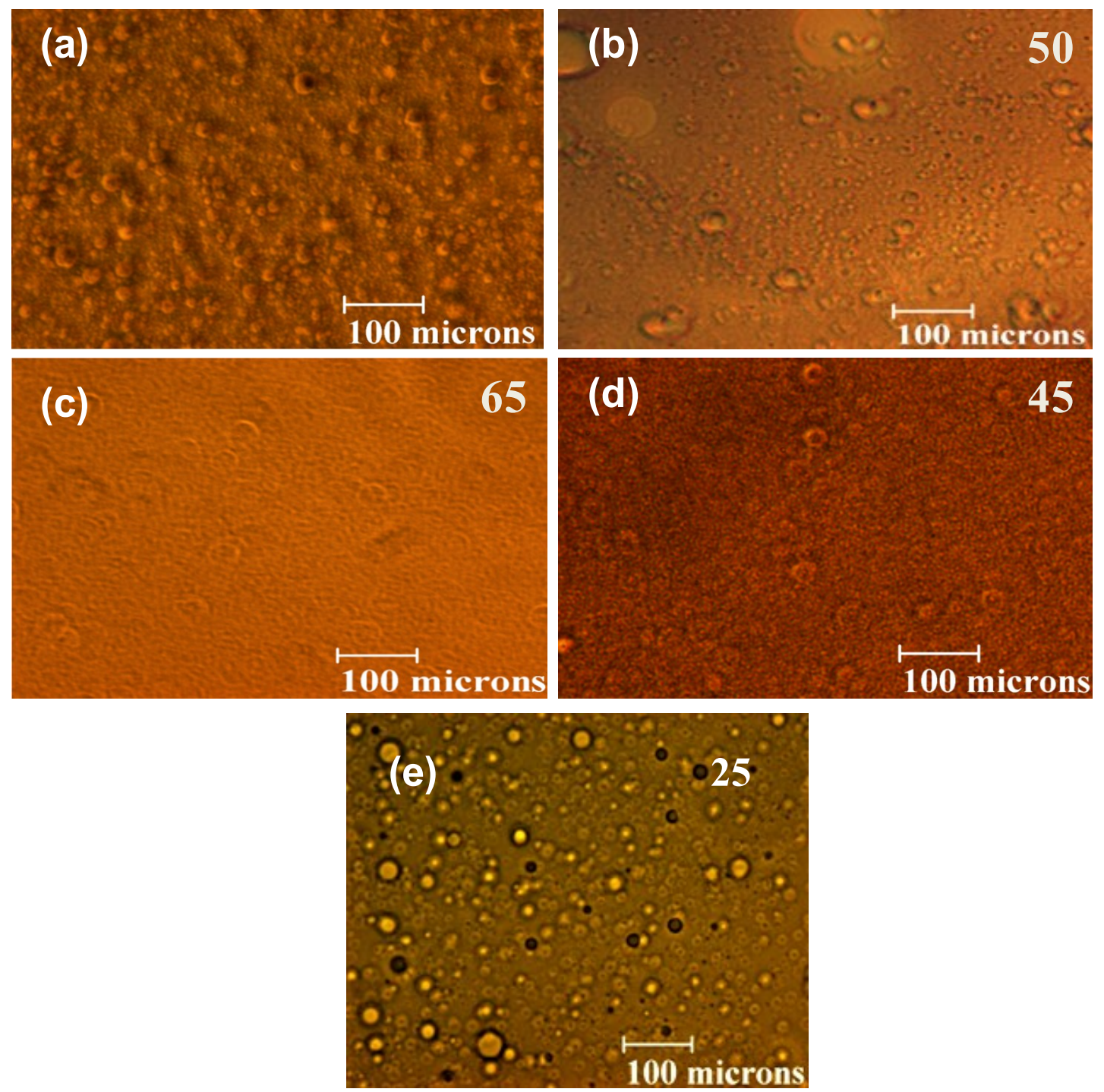

Figure 3.10 Optical micrographs of (a) gel at room temperature (b) at $50{ }^{\circ} \mathrm{C}$; (c) at $65{ }^{\circ} \mathrm{C}$ showing the transition to sol; (d) at $45^{\circ} \mathrm{C}$ and (e) at $25^{\circ} \mathrm{C}$ with the spherical domains reformed

\subsubsection{Mechanical Properties}

The variations of G' and G' with frequency are shown in Figure 3.11a for the bulk sample of PDMS, the gel and the xerogel. It is seen that for the PDMS gel, the G' is higher than the G" and remains almost invariant through the frequency range. This confirms that the material is indeed a gel. The xerogel shows higher moduli than the gel and in this case also the G' is higher. However, for the bulk PDMS, G' is higher than G' over the frequency range, and both 
moduli increase with frequency. Thus, a clear distinction is seen between the gels and the bulk PDMS materials. The thermo-reversibility of the gel is seen in the rheological measurements as well. For this experiment, the gel sample was heated from 25 to $70{ }^{\circ} \mathrm{C}$ (continuous ramp), at a slow rate of $0.15 \mathrm{deg} / \mathrm{min}$, with a frequency of $1 \mathrm{~Hz}$. The edge of the parallel plate was sealed with wax to prevent solvent evaporation. The same rate was used for cooling the sample back to $25^{\circ} \mathrm{C}$. It is seen from Figure $3.11 \mathrm{~b}$ that upon increasing the temperature, a transition occurs in $\mathrm{G}$ ' between 50 and $60{ }^{\circ} \mathrm{C}$, and that it is fully reversible. 


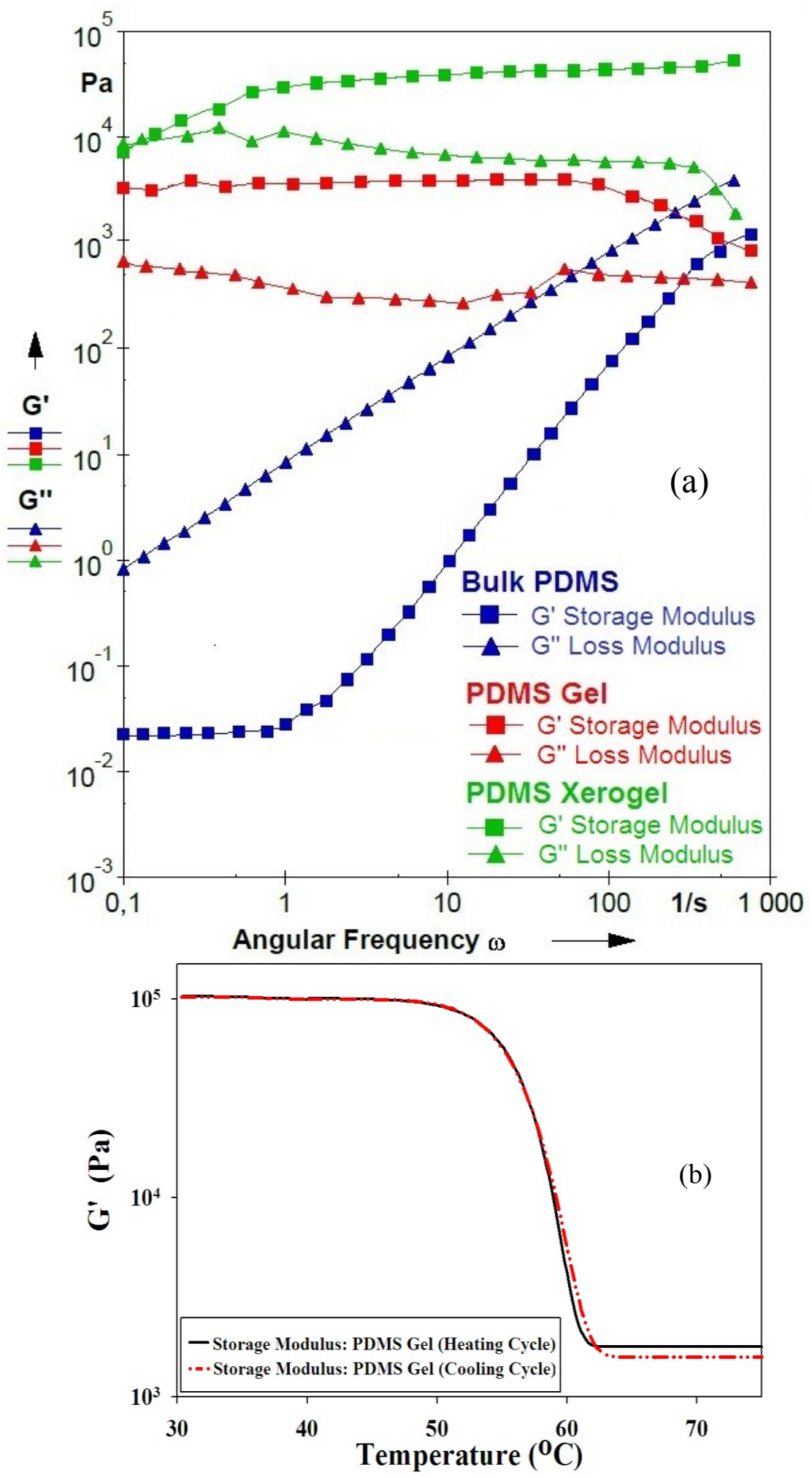

Figure 3.11 (a) Variation of G' and G' with frequency for PDMS gel, xerogel and the bulk PDMS (b) Thermo-reversibility of G' of the PDMS gel 
Further evidence for the existence of network connectivity between the spherical domains is indicated by a simple stretching test. We used a manual stretching device (Figure 3.12) to test the elongation of the original PDMS sample (which flows as seen in Figure 3.1) and the gel. The elongation ratio was calculated as

$$
\lambda=\left(\mathrm{L}-\mathrm{L}_{0}\right) / \mathrm{L}_{0}
$$

where $\mathrm{L}_{0}$ and $\mathrm{L}$ are initial and final lengths, respectively. We used $3 \mathrm{mg}$ of the bulk PDMS or the gel sample, and set the initial separation of the edges of the stretcher to $0.05 \mathrm{~cm}$. The samples were stretched slowly, and ten sets of measurements were performed. As seen in Figure 3.12, the original PDMS sample can be stretched to a large extent, with an elongation ratio of $\lambda=57 \pm 4$, while the gel, due to the network formation, resists stretching. Its elongation ratio $(\lambda)$ is $7.8 \pm 1.7$.

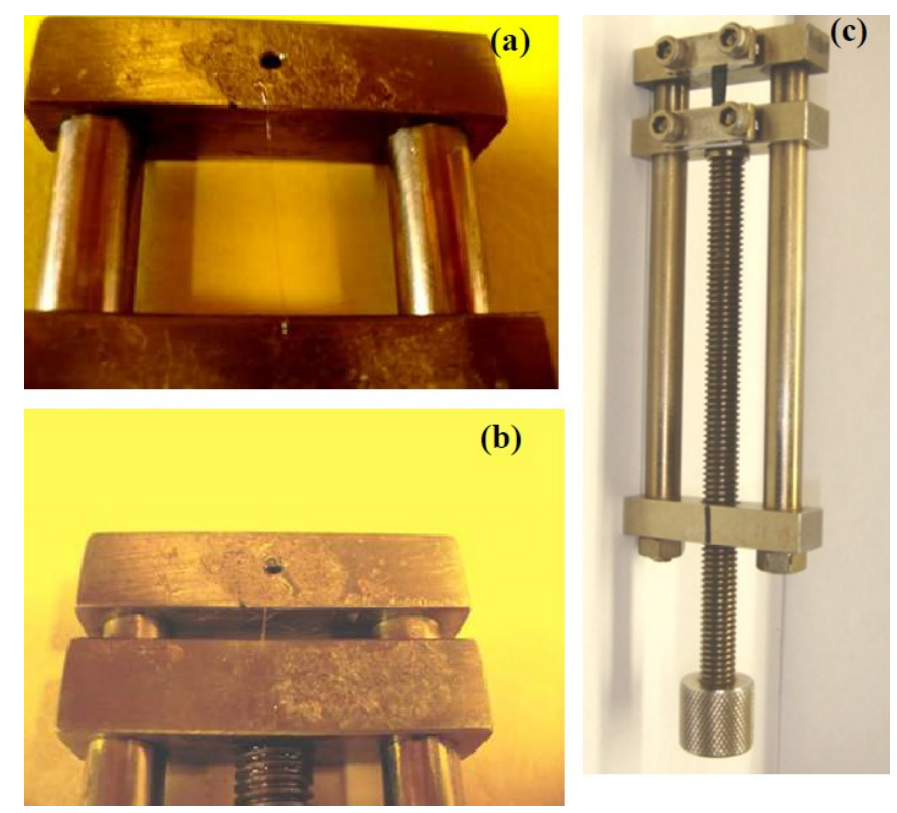

Figure 3.12 Stretching of (a) original PDMS high molecular weight sample and (b) the gel

(c) photograph of the manual stretcher used 
Yet another test using a piston was suggested by Guenet ${ }^{1,2}$ to distinguish between a gel and a paste. Following his pictorial representation, we used a common laboratory syringe with the nozzle sealed, to compress the material and note its relaxation upon slow withdrawal of the piston. The intent here is to show the difference, and not to derive a quantitative measurement. Figure 3.13 shows the photographs of the compressed gel (top right) and after withdrawal of the piston head (bottom right). The gel completely recovered upon release of the stress, confirming that it is a gel and not a paste. In contrast, the bulk PDMS, upon withdrawal of the piston remained in its compressed location, and did not recover.

Bulk PDMS
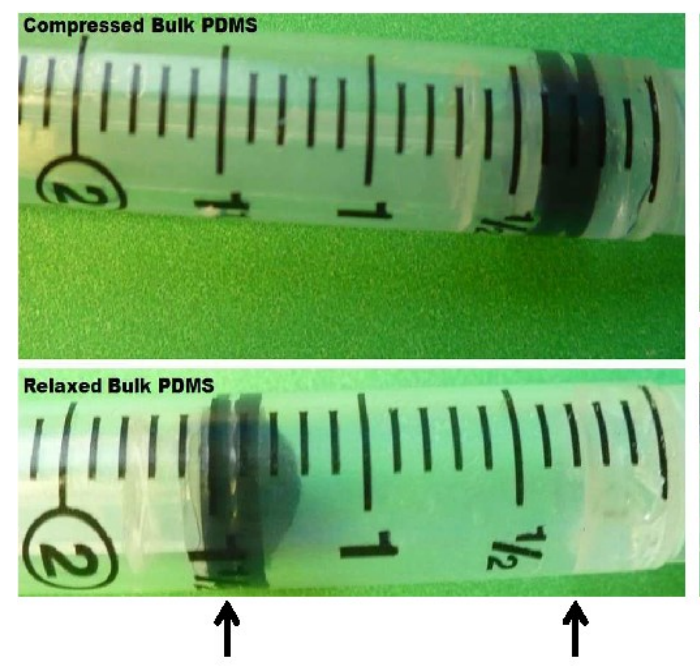

Piston Head Sample Edge
PDMS Gel

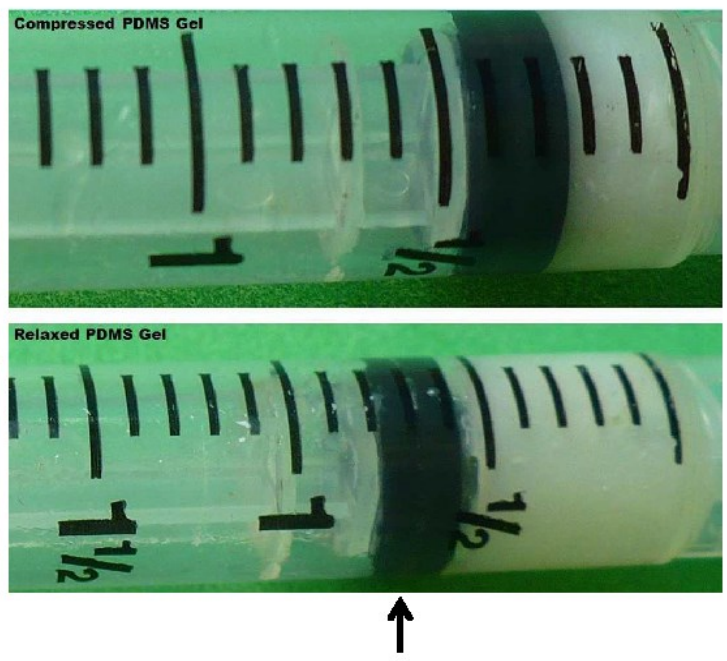

Piston Head

Figure 3.13 Compression and relaxation of (left) the original high Mw bulk PDMS and (right) the gel. The top photos show the compressed sample and the bottom, after withdrawal of the piston. Note that the gel recovered from the compressed state

\subsubsection{Organogel of Low Molecular Weight PDMS}

The gel with the PDMS of low molecular weight $(\mathrm{Mw}=323$, with just four skeletal atoms $)$ can be classified as an organogel. This sample showed a different morphology upon gelling, as 
shown in Figure 3.14 a. Rods of about $50 \mu \mathrm{m}$ in length are seen, which would indicate that with this molecular weight, the molecules simply aggregated in an extended fashion. A model of the aggregation is shown in Figure $3.14 \mathrm{~b}$. The birefringence seen in Figure $3.14 \mathrm{a}$ is due to amorphous orientation, and X-ray diffraction confirmed that these are not crystalline at room temperature. In our previous study ${ }^{54}$ this sample, from the bulk, formed single crystals upon cooling to a temperature of $-100{ }^{\circ} \mathrm{C}$. Thermo-reversibility was confirmed in this case also with DSC scans as shown in Figure 3.15.
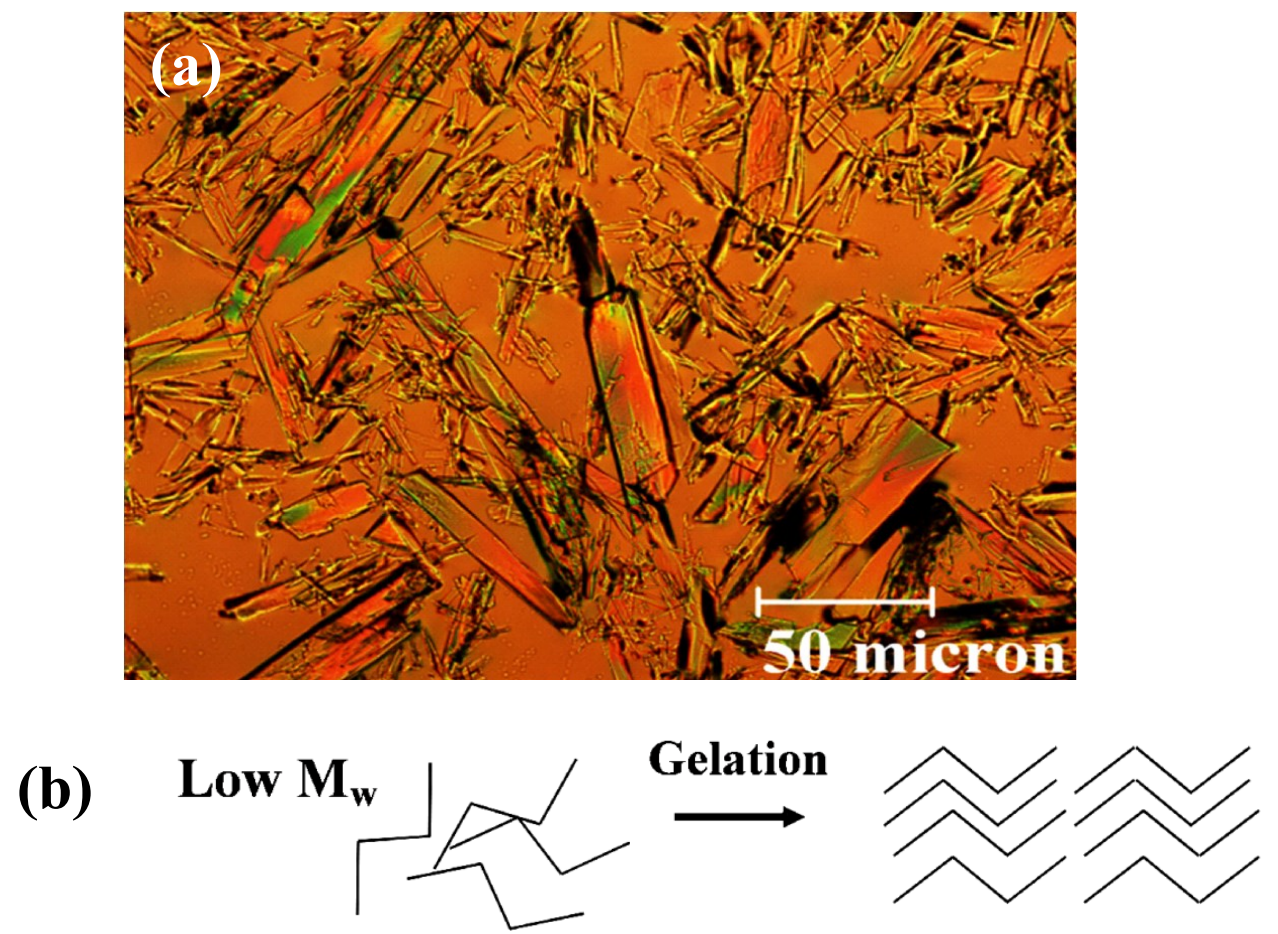

Figure 3.14 (a) Optical micrograph of the gel with low molecular weight PDMS (b) formation of rods during the organo gelation of low Mw PDMS 


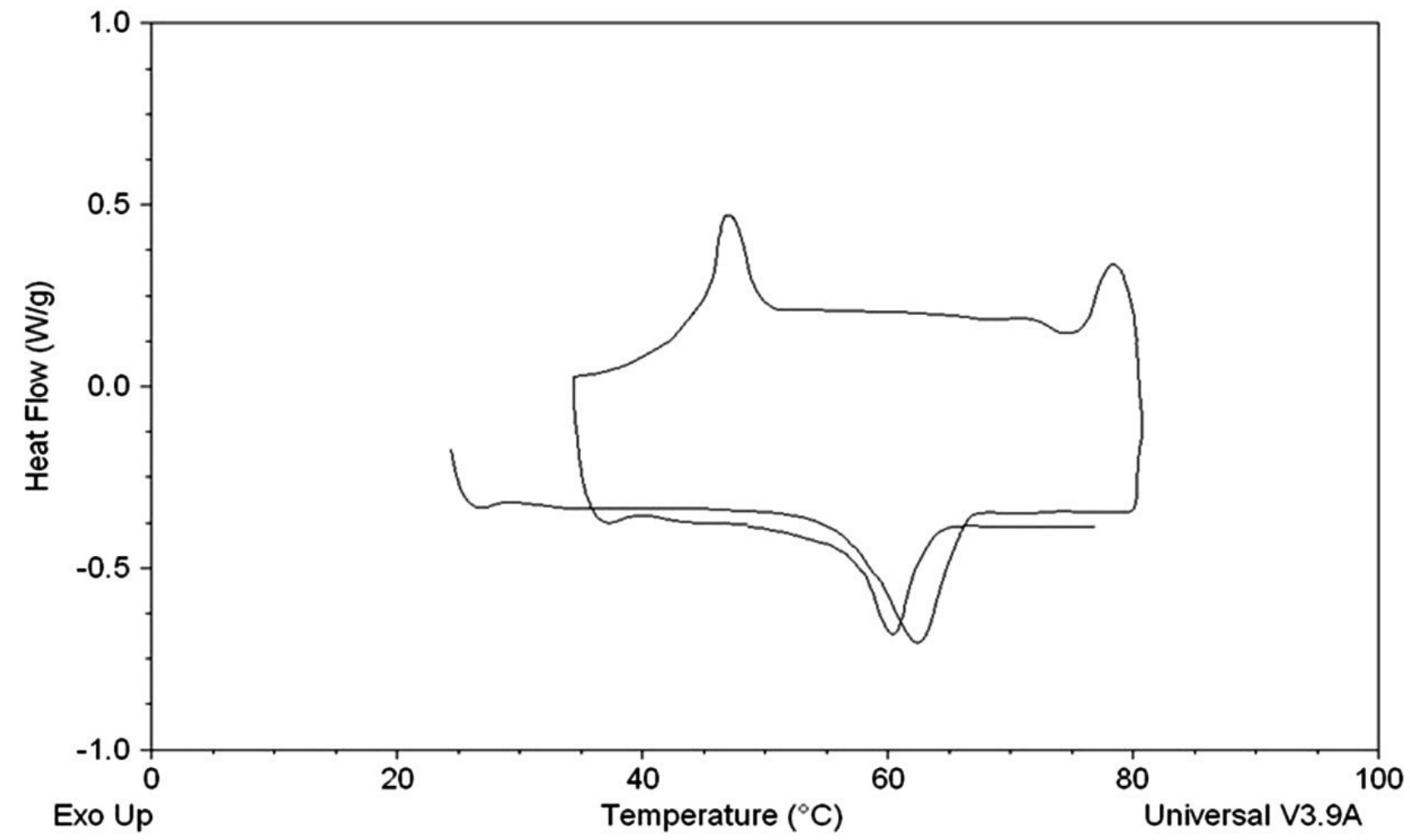

Figure 3.15 DSC trace of low Mw PDMS during the heating, cooling and second heating cycles. The sample was kept for 45 minutes at $45^{\circ} \mathrm{C}$ during the cooling cycle to enhance the gel formation 


\subsection{Conclusions}

In this chapter, we have shown that thermo-reversible physical gels of PDMS can be prepared with propyl amine or hexylamine as the solvent, without any catalysts and crosslinks or functionalization. The morphology of the gels consists of spherical domains. Due to the low $\mathrm{T}_{\mathrm{g}}$ of the polymer and the tendency for adopting conformations analogous to macrocyclization, spherical domains result rather than fibrous networks. A low molecular weight sample $(\mathrm{Mw}=$ 323, with just four skeletal atoms) formed organogels with propylamine, with a rod-like morphology. We have demonstrated that the spherical domains formed with the high Mw PDMS are part of a network, as seen from the E-SEM images of the fracture domain and the TEM micrograph. The gelation and thermo-reversibility is demonstrated by DSC, OM and rheology. With the DSC experiments, we have devised a method to achieve thermo-reversibility, with very similar gel-sol transition endotherms in the first and second heating cycles. A simple compression experiment confirmed that these are gels and not pastes. With the DSC measurements, after the first heating cycle (in which a well- defined gel-sol endotherm is seen) it was necessary to keep the sample at the sol-gel temperature for about 30 minutes during the cooling cycle, in order to obtain an endotherm during the second scan, which is comparable to that in the first heating cycle. These procedures might be useful in thermo-reversibility studies of other systems. The simple route to physical gels of PDMS might be extended to preparing particle filled PDMS and other PDMS based composite gels. 


\subsection{References}

1. Guenet, J.M. Thermo-reversible Gelation of Polymers and Biopolymers. London: Academic Press, 1992.

2. Guenet, J.M. Polymer Thermo-reversible Gels vs Organogels. Macromol Symp. 2006, $241,45-50$.

3. Keller, A. Aspects of Polymer Gels. Faraday Discuss. 1995, 101, 1-49.

4. Terech, P.; Weiss, R.G. Low Molecular Mass Gelators of Organic Liquids and the Properties of Their Gels. Chem. Rev. 1997, 97, 3133-3159.

5. Guenet, J.M.; Wittmann, J.C.; Lotz, B. Thermodynamics Aspects and Morphology of Isotactic Polystyrene Physical Gels. Macromolecules 1985, 18, 420-427.

6. Sundararajan, P.R.; Tyrer, N.; Bluhm, T. Solvent Dependent Conformations in Gels of Isotactic Polystyrene. Macromolecules 1982, 15, 286-290.

7. Atkins, E.D.T.; Isaac, D.H.; Keller, A.; Miyasaka, K.J. Analysis of Anomalous X-ray Diffraction Effects of Isotactic Polystyrene Gels and its Implications for Chain Conformation and Isomeric Homogeneity. Polym. Sci. Polym. Phys. Ed., 1977, 15, 211-226.

8. Girolamo, M.; Keller, A.; Miyasaka, K.; Overbergh, N.J. Gelation-Crystallization in Isotactic Polystyrene Solutions and its Implications to Crystal Morphology, to the Origin and Structure of Gels, and to the Chemical Homogeneity of Polyolefins. Polym. Sci. Polym. Phys. Ed. 1976, 14, 39-61.

9. Atkins, E.D.T.; Isaac, D.H.; Keller, A.J. Conformation of Polystyrene with Special Emphasis to the Near All-trans Extended-Chain Model Relevant in Polystyrene Gels. Polym. Sci. Polym. Phys. Ed. 1980, 18, 71-82. 
10. Wellinghoff, S.; Shaw, J.; Baer, E. Polymeric Materials from the Gel State. The Development of Fringed Micelle Structure in a Glass. Macromolecules 1979, 12, 932939.

11. Luo, Y.; Kayakabe, S.; Xi, Y.; Bin, Y.; Xu, C. Matsuo, M. Characteristics of Several Kinds of Polyethylene Gel Estimated by Small-Angle Light Scattering Under Cross Polarization. Polym. Sci. Part B: Polym. Phys. 2011, 49, 384-397.

12. Sawatari, C.; Okumura, T.; Matsuo, M. Molecular Weight Dependence on the Morphological Properties of Polyethylene Gels. Polym J. 1986, 18, 741-758.

13. Silicon-Containing Polymers: The Science and Technology of Their Synthesis and Applications. Jones, R.G.; Ando,W.; Chojnowski, J. Eds. Kluwer Academic Publishers, 2001.

14. Silicon-Based Polymer Science: A Comprehensive Resource. Zeigler, J.M.; Fearon, F.W.G. Eds. 1989 Advances in Chemistry Series 224. American Chemical Society.

15. Mark, J.E.; Allcock, H.R.; West, R. Inorganic Polymers, Oxford University Press, 2005.

16. Silicon Polymers. Muzafarov, A.M. Ed. Advances in Polymer Science 235. Springer, 2010.

17. Tomanek, A. Silicones and Industry. Munich: Hanser (Wacker Chemie), 1991.

18. Nguyen, Q.T.; Nobe, K. Extraction of Organic Contaminants in Aqueous Solutions by Pervaporation. Membrane Sci. 1987, 30, 11-22.

19. Cai, D.; Neyer, A.; Kuckuk, R.; Heise, H.J. Raman, Mid-Infrared, Near-Infrared and Ultraviolet-Visible Spectroscopy of PDMS Silicone Rubber for Characterization of Polymer Optical Waveguide Materials. Molec. Struct. 2010, 976, 274-281. 
20. Geissler, E.; Horkayt, F.; Hecht, A.M.; Rochas, C. Investigation of PDMS Gels and Solutions by Small-Angle Scattering. Polymer. 1997, 38, 15-20.

21. Ye, H.; Gu, Z.; Gracias, D.H. Kinetics of Ultraviolet and Plasma Surface Modification of Poly(dimethylsiloxane) Probed by Sum Frequency Vibrational Spectroscopy Langmuir, 2006, 22, 1863-1868.

22. Mahltig, H.B.; Haufe ,H.; Bottcher, H.J. Functionalisation of Textiles by Inorganic Sol-gel Coatings. Mater. Chem. 2005, 15, 4385-4398.

23. Falcâo, A.N.; Carrapico, M.J.; Sousa, S. SANS Study of Zirconia-Silica and TitaniaSilica Hybrid Materials. J. Sol-Gel Sci. Technol., 2003, 26, 349-352.

24. Falcâo, A.N.; Pedersen, J.S.; Mortensen, K. Structure of Randomly Cross-linked Poly(dimethylsiloxane) Networks Produced by Electron Irradiation. Macromolecules 1993, 26, 5350-5364.

25. Braun, J.L.; Mark, J.E; Eichinger, B.E. Formation of Poly (dimethysiloxane) Gels. Macromolecules 2002, 35, 5273-5282.

26. Yu, T.; Wakuda, K.; Blair, D.L.; Weiss, R.G. Reversibly Crosslinking AminoPolysiloxanes by Simple Triatomic Molecules. Facile Methods for Tuning Thermal, Rheological, and Adhesive Properties. Polym. Preprints 2009, 50(2), 118-119.

27. Hajsz, T.; Csetneki, I.; Filipcsei, G.; Zrinyi, M. Swelling Kinetics of Anisotropic Filler Loaded PDMS Networks. Phys. Chem. Chem. Phys., 2006, 8, 977-984.

28. Sertchook, H.; Elimelech, H.; Avnir, D. Composite Particles of Silica/ Poly(dimethylsiloxane). Chem. Mater. 2005, 17, 4711- 4716. 
29. Dewimille, L.; Bresson, B.; Bokobza, L. Synthesis, Structure and Morphology of Poly (Dimethylsiloxane) Networks Filled with in situ Generated Particles. Polymer 2005, $46,4135-4143$.

30. Suzuki, M.; Owa, S.; Shirai, H.; Hanabusa, K.J. Poly(dimethylsiloxane)-Based Polymer Organogelators with L-lysine Derivatives as a Organogelation-Causing Segment. Polym. Sci. Polym. Chem. 2006, 44, 3817-3824.

31. Van Esch, J.H. We Can Design Molecular Gelators, But Do We Understand Them? Langmuir 2009, 25, 8392- 8394.

32. Khanna, S.; Khan, M.K.; Sundararajan, P.R. Influence of Double Hydrogen Bonds and Alkyl Chains on the Gelation of Nonchiral Polyurethane Model Compounds: Sheets, Eaves Trough, Tubes and Oriented Fibers. Langmuir 2009, 25, 13183-93.

33. Moniruzzaman, M.; Sundararajan, P.R. Low Molecular Weight Organogels Based on Long-Chain Carbamates. Langmuir 2005, 21, 3802-3807.

34. Hikmet, R.M.; Callister, S.; Keller, A. Thermo-reversible Gelation of Atactic Polystyrene: Phase Transformation and Morphology. Polymer 1988, 29, 1378-1388.

35. Xu, H.; Song, J.; Tian, T.; Feng, R. Estimation of Organogel Formation and Influence of Solvent Viscosity and Molecular Size on Gel Properties and Aggregate Structures, Soft Matter 2012, 8, 3478-3486.

36. Feng, L.; Cavicchi, K. A. Investigation of the Relationships Between the Thermodynamic Phase Behaviour and Gelation Behavior of a Series of Tripodal Trisamide Compounds. Soft Matter 2012, 8, 6483-6492.

37. Raynal, M.; Bouteiller, L. Organogel Formation Rationalized by Hansen Solubility Parameters. Chem Commun. 2011, 47, 8271-8273. 
38. Das, D.; Kar, T.; Das, P. K. Gel-Nanocomposites: Materials with Promising Applications. Soft Matter 2012, 8, 2348-2365.

39. Vaysse,M.; Khan, M. K.; Sundararajan, P. Carbon Nanotube Reinforced Porous Gels of Poly(methyl methacrylate) with Nonsolvents as Porogens. Langmuir 2009, 25, 7042-7049.

40. Lee, J. N.; Park, C.; Whitesides, G. M. Solvent Compatibility of Poly(dimethylsiloxane)-Based Microfluidic Devices. Anal. Chem. 2003, 75, 65446554.

41. Flory, P. J. Statistical Mechanics of Chain Molecules.1969, John Wiley and Sons, N. Y.

42. (a) Morgan, A. M.; Pollack, S. K.; Beshah, K. Synthesis and Multidimensional NMR Characterization of PDMS- $b$-PS Prepared by Combined Anionic Ring-Opening and Nitroxide-Mediated Radical Polymerization. Macromolecules 2002, 35, 4238-4246.

(b) Su, F-H.; Shyu, S-S.; Chen, Y-C. NMR Properties of Poly(dimethyl siloxane) Colloids as New Contrast Agents for NMR Imaging, Polym. Int. 2000, 49, 787-794 (c) O’Neill, I. K.; Pringuer, M. A.; Prosser, H. J. Comparison of the Nuclear Magnetic Resonance and Infrared Spectroscopic Methods for the Quantitative Analysis of Polydimethylsiloxane. J. Pharm. Pharmacology 1975, 27, 222-225.

43. Mark, J. E. Dipole Moments of Dimethylsiloxane Chains, J. Chem. Phys. 1968, 49, 1398-1402.

44. Jiang, K.; Thomas, P. C.; Forry, S. P.; De Voe, D. L.; Raghavan, S. R. Microfluidic Synthesis of Monodisperse PDMS Microbeads as Discrete Oxygen Sensors. Soft Matter 2012, 8, 923-926. 
45. Perro, A.; Nicolet, C.; Angly, J.; Lecommandoux, S.; Le Meins, J-F.; Colin, A. Mastering a Double Emulsion in a Simple Co-Flow Microfluidic to Generate Complex Polymersomes. Langmuir 2011, 27, 9034-9042.

46. Harth, E.; Van Horn, B.; Lee, V. Y.; Germack, D. S.; Gonzales, C. P.; Miller, R. D.; Hawker, C. J. A Facile Approach to Architecturally Defined Nanoparticles via Intramolecular Chain Collapse J. Am. Chem. Soc. 2002, 124, 8653-8660.

47. Bibette, J.; Mason, T. G.; Gang, H.; Weitz, D. A. Kinetically Induced Ordering in Gelation of Emulsions. Phys. Rev. Lett. 1992, 69, 981-984.

48. Flory, P.J.; Crescenzi, V.; Mark, J.E. Configuration of the Poly-(dimethylsiloxane) Chain. III. Correlation of Theory and Experiment J. Am. Chem. Soc.1964, 86,146-152.

49. Flory, P. J.; Semlyen, J. A. Macrocyclization Equilibrium Constants and the Statistical Configuration of Poly ( dimethylsiloxane) Chains J. Am. Chem. Soc., 1966, 88, 32093212.

50. Damaschun, G. RiJntgenographische Untersuchung der Struktur von Silikongummi Kolloid-Z., 1962, 180, 65-67.

51. Flory, P. J.; Mandelkern, L.; Kinsinger, J. B.; Shultz, W. B. Molecular Dimensions of Polydimethylsiloxanes. J. Am. Chem. Soc. 1952, 74, 3364-3367.

52. Beltzung, M.; Picot, C.; Rempp, P.; and Herz, J. Investigation of the Conformation of Elastic Chains in Poly(dimethylsiloxane) Networks by Small-Angle Neutron Scattering. Macromolecules 1982, 15, 1594-1600.

53. Jadav, G. L.; Aswal, V. K.; Singh, P. S. Characterization of Polydimethylsiloxane Pervaporation Membranes Using Small-Angle Neutron Scattering. J. Membrane Sci. 2011, 378, 194-202.

54. Sundararajan, P.R. Polymer 2002, 43, 1691. 


\section{CHAPTER 4}

\section{Thermo-reversible Gelation of Rod-Coil and Coil-Rod-Coil Molecules Based on Poly (Dimethyl Siloxane) and Perylene Imides without Hydrogen Bonding Groups}

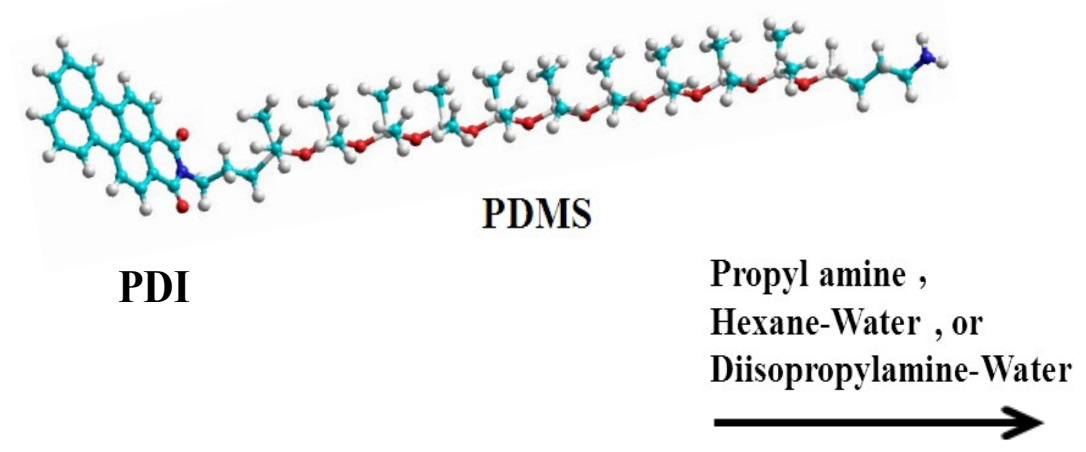

Heat/ Slow-cool

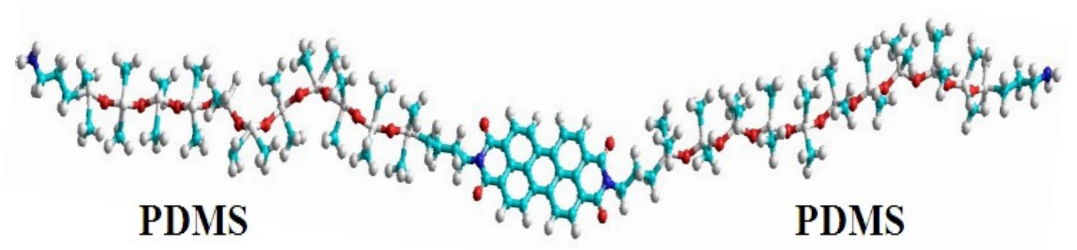

PTCDI
Thermo-reversible Gel
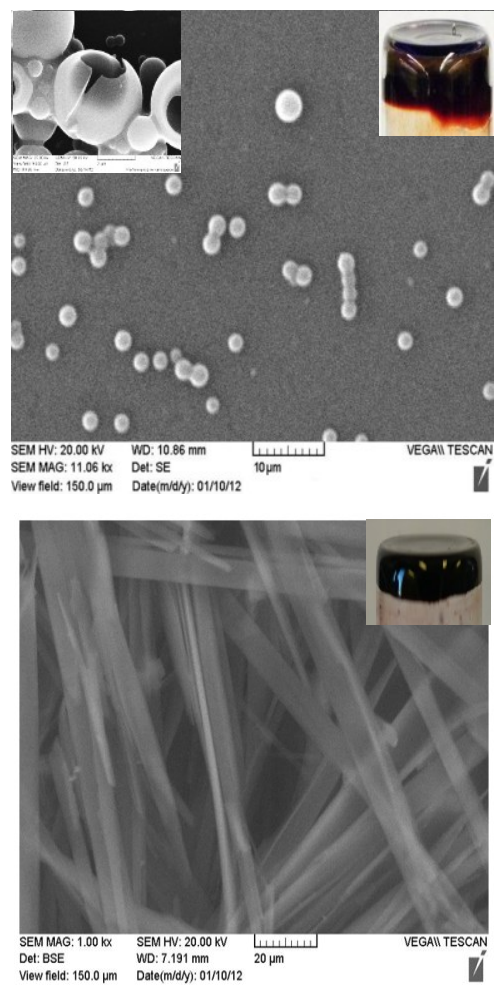


\subsection{Introduction}

Perylene tetracarboxylic diimides (PTCDI) have been the subject of extensive research during the past few decades due to their applications as electronic materials in xerographic photoreceptors $^{1}$ and photovoltaics ${ }^{2}$. The optical absorption properties depend on the $\pi$-overlap between adjacent molecules. Through an extensive crystallographic study on perylene derivatives, Hädicke and Gracer related the effect of peripheral substituents on the extent of $\pi$ overlap and the color of the pigments. ${ }^{3}$ The structural and photo-physical properties can thus be tuned by substitutions at the imide as well as the bay positions. The $\pi$-interaction mediated selfassembly of the perylene segment would then be influenced by the substituent. Some of the short linear substituents lead to liquid crystalline behavior. ${ }^{4}$ Linear and forked substituents have been shown to give rise to nano-fiber morphologies. ${ }^{5,6}$ Supramolecular polymers with nanoribbon and nanorope morphologies were created by functionalizing PTCDI with melamine and forming hydrogen bond complex with cyanuric acid. ${ }^{7}$ Likewise, hydrogen bonded lamellar organization of soluble PTCDI has been reported by Seki et al. ${ }^{8}$ Our group previously reported the structure and morphologies of perylene imides substituted with poly (dimethylsiloxane) (PDMS) either on one imide position (Mono-PDMS) or on both positions (Di-PDMS), ${ }^{9,10}$ as shown in Scheme 1. (We denote PDMS-PDI and PDMS-PTCDI-PDMS as Mono-PDMS and Di-PDMS, respectively in this paper). These are examples of rod-coil and coil-rod-coil systems, with the rod being a photoactive molecule with a large aromatic structure. Since we used PDMS segments with 10, 20 and 40 repeat units, this substitution is longer than any of those cited above. The Mono-PDMS is an inverse macromolecular surfactant (head group is insoluble) relevant to non-aqueous systems, and the Di-PDMS is similar to a Gemini surfactant (about the axis of symmetry) as indicated in Scheme 1. Similar to amphiphilic block copolymer systems, micellar or vesicular morphologies 
were observed with Mono-PDMS, depending on the block specific solvent used. ${ }^{9}$ The Di-PDMS molecule exhibited a nanoweb morphology. ${ }^{10}$ Attachment of such longer chains to PTCDI impedes liquid crystalline ordering.

An organogel is a state of matter that has seen an extensive exploration in the past few years as to the design, properties and possible applications. ${ }^{11-19}$ This is essentially the entrapment of a solvent in the physical network structure resulting from the self-assembly of organic small molecules facilitated by non-covalent interactions such as hydrogen bonding and $\pi \ldots \pi$ interactions. While those involving hydrogen bonds have been studied by several authors, $\pi$ interaction mediated organogel based on oligo-p-phenylenevinylenes (OPV) was first reported by Ajayaghosh and George. ${ }^{20,21}$ Since then, several PTCDI derivatives as gelators have also been reported. Shinkai et $\mathrm{al}^{22}$ prepared several gelators by connecting cholesterol groups to imide positions of PTCDI. The compounds could form a gel in a mixed solvent of p-xylene and 1propanol. Würthner and co-workers reported highly fluorescent organogels based on several PTCDI derivatives ${ }^{23,24}$. Recently, Wu et $\mathrm{al}^{25}$ described the gels based on PTCDI substituted with short linear hydrophilic and hydrophobic segments, as well as bay-substitution, in mixed solvents. Phthalocyanine based organogels were reported by Diaz et al. ${ }^{26,27}$

In all the $\pi$-system based gels mentioned above, including the OPV, in addition to the self-assembly promoted by $\pi-\pi$ interaction, the gelation seems to have been assisted by hydrogen bonding as well. The above authors inserted hydrogen bonding groups in the substitution design of the PTCDI. In this paper, we describe the gelation of two $\pi$-systems, where there is no hydrogen bonding involved. The Mono-PDMS and Di-PDMS described above, form thermoreversible gels with propylamine. The gelation is driven by the $\pi$ interaction and the polymer segment. Another aspect of this work is as follows: it is usual in the studies on gelation that if a 
solvent dissolves a solute at room temperature, the latter is not considered a prospective gelator for that solvent. We find, however, that Mono-PDMS and Di-PDMS can gel hexane and diisopropylamine, although these are good solvents at room temperature. Yet another aspect of this paper is that with Mono-PDMS, the perylene is mono-substituted, and gelation involving such mono-substituted perylene imide has not been reported to date. The thermo-reversibility, morphology, and optical absorption properties are discussed.

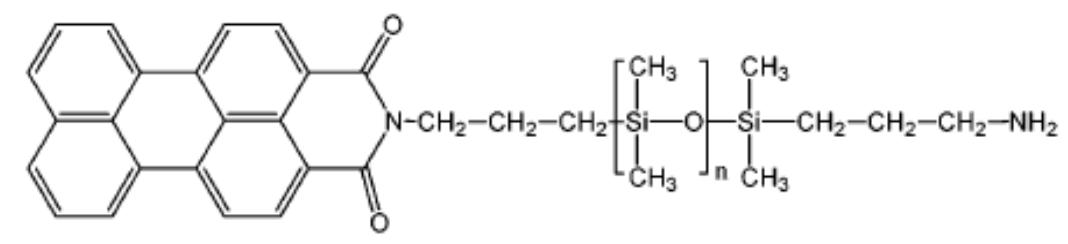

(a)

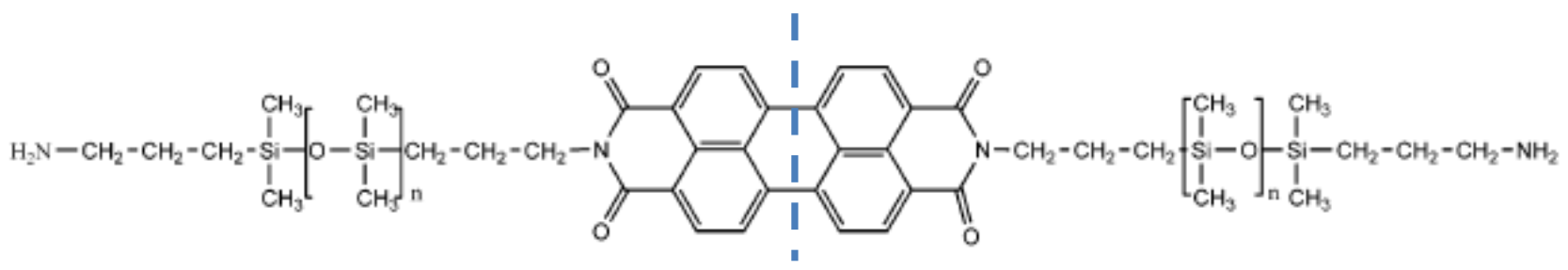

(b)

\section{Scheme 1. (a) Mono-PDMS (b) Di-PDMS. $n=10,20$ or 40. The dashed line indicates the symmetry on either side that render the molecule a (non- aqueous) Gemini surfactant}

\subsection{Experimental}

Amine-terminated poly (dimethylsiloxane)s (DMS-A11, DMS-A12, and DMS-A15) were purchased from Gelest Inc. and used as received. Their molecular weights were determined by Yao et al to be 875,1500 , and 3035, respectively. The synthesis of Mono-PDMS and DiPDMS was described before. ${ }^{9,10}$ Weighed amounts of Di-PDMS and Mono-PDMS $\left(\mathrm{M}_{\mathrm{w}}\right.$ of PDMS: $1500 ; 3035)$ of different concentrations $(3-10 \mathrm{mM})$ were dissolved in the required 
amount of propylamine at temperatures ranging from $50-80{ }^{\circ} \mathrm{C}$ with constant stirring. Closed vials were used to avoid the evaporation of the solvent. This resulted in a red solution. The gels were prepared by slowly cooling the solution to room temperature. Gelation was confirmed by tube inversion, i.e., no flowing solvent. Xerogels were prepared by drying the gels at room temperature for a few days and then under vacuum overnight for complete removal of propylamine. The same procedure was followed to prepare gels from hexane/ water and diisopropylamine/ water mixtures, at different water concentrations ranging from $5-15 \%$.

\subsubsection{Results and Discussion}

The PDMS attached to PTCDI or PDI here is 20 or 40 monomer units long $\left(\mathrm{M}_{\mathrm{w}}=1500\right.$ or 3035), and is longer than most substituents used so far for perylene imides. Although PDMS is an amorphous polymer, the self-assembly of the perylene imide was evident from the crystalline X-ray diffraction reported before. ${ }^{9}$ In a previous publication ${ }^{28}$, we discussed the gelation of PDMS/ propylamine system, without any crosslinks or functionalization of PDMS. Both MonoPDMS and Di-PDMS are insoluble in propylamine at ambient temperatures. Upon heating to 65$70{ }^{\circ} \mathrm{C}$, clear solutions were obtained for both Mono-PDMS and Di-PDMS. Upon cooling the solutions, gelation occurs. Figure 4.1 shows the effect of concentration on the temperatures of dissolution, onset of gelation and completion of gelation, respectively. For example with $8.0 \mathrm{mM}$ concentration, a transparent orange solution was obtained at $68^{\circ} \mathrm{C}$. When the solution was slow cooled, a deep red-colored, opaque and immobile gel began to form at $59{ }^{\circ} \mathrm{C}$. It is seen that these temperatures are similar for both Mono- and Di-PDMS. The inset in Figure 4.1 shows the inverted vials containing the gels. The minimum concentration required for gelation is $3.0 \mathrm{mM}$. 

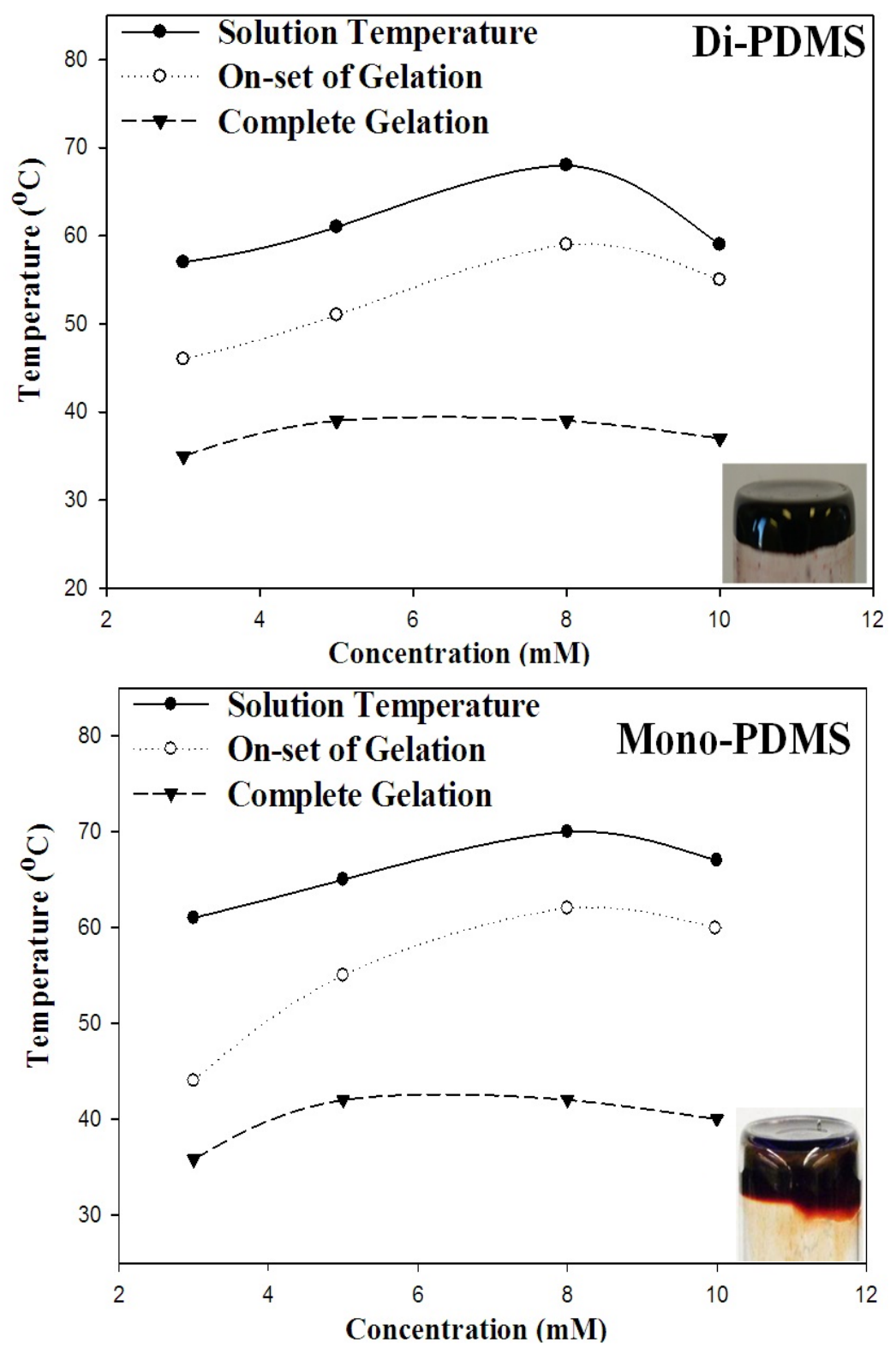

Figure 4.1 Solution and Gelation Temperatures of (a) Di-PDMS (b) Mono-PDMS ( $\left.\mathrm{M}_{\mathrm{w}}: 1500\right)$. The insets show the inverted vials containing the gels

Whitesides et $\mathrm{al}^{29}$ found hexane and diisopropylamine to be good solvents for PDMS. The solubility parameter of the two solvents is the same as that of PDMS $\left(7.3 \mathrm{cal}^{1 / 2} \mathrm{~cm}^{-3 / 2}\right)$. These solvents dissolve both Mono- and Di-PDMS at room temperature and would not normally qualify as gelators. If water (a non-solvent) is added to the solution, precipitation occurred, as 
one would expect. We serendipitously found that upon heating the solution of Mono- or DiPDMS in hexane or diisopropylamine, cloudiness developed at about $70{ }^{\circ} \mathrm{C}$ indicating phase separation. Adding water $(5-15 \%(w t))$ at this stage and then cooling to room temperature resulted in a gel. Figure 4.2 shows the temperature dependence of the diisopropylamine and hexane solutions. Without the addition of water, precipitation occurred if the solution was quenched or slowly cooled to room temperature. Heating the solute in a good solvent and forming a gel is a new and unexpected result, but also raises the question as to the cause for such a behavior. We offer a tentative reasoning as follows: PDMS by itself, in a solution of hexane, does not phase separate upon heating to $70{ }^{\circ} \mathrm{C}$. Di- or Mono-PDMS dissolve in hexane or diisopropylamine at room temperature. Upon heating, it is likely that PTCDI or PDI begins to aggregate at about $70{ }^{\circ} \mathrm{C}$. Upon cooling, the perylene aggregates do not go back into solution, and precipitation occurs. However, if water is added at $70{ }^{\circ} \mathrm{C}$, it collapses the PDMS segment as well. The entanglement of the PDMS segment as well as the aggregation of perylene leads to gelation.

The DSC analysis (Figure 4.3) of both Di-PDMS and Mono-PDMS gels showed an endotherm at $65{ }^{\circ} \mathrm{C}$ in the first heating cycle corresponding to the gel-sol transition, and a sol-gel transition exotherm at $41^{\circ} \mathrm{C}$ upon cooling, and a gel-sol transition during the second heating cycle. This confirms the thermo-reversibility of the gels. A well-defined transition, at $67^{\circ} \mathrm{C}$ is seen in the second heating cycle. Often, only a shallow transition would be seen in the second heating cycle in the case of gels. As in our previous work ${ }^{28}$, we kept the sample at $45^{\circ} \mathrm{C}$ for 30 minutes during the cooling cycle to enable sufficient gel formation. This causes the endotherm in the second heating cycle to be as well-defined as the one in the first cycle. We show such reversibility using optical microscopy as well. 

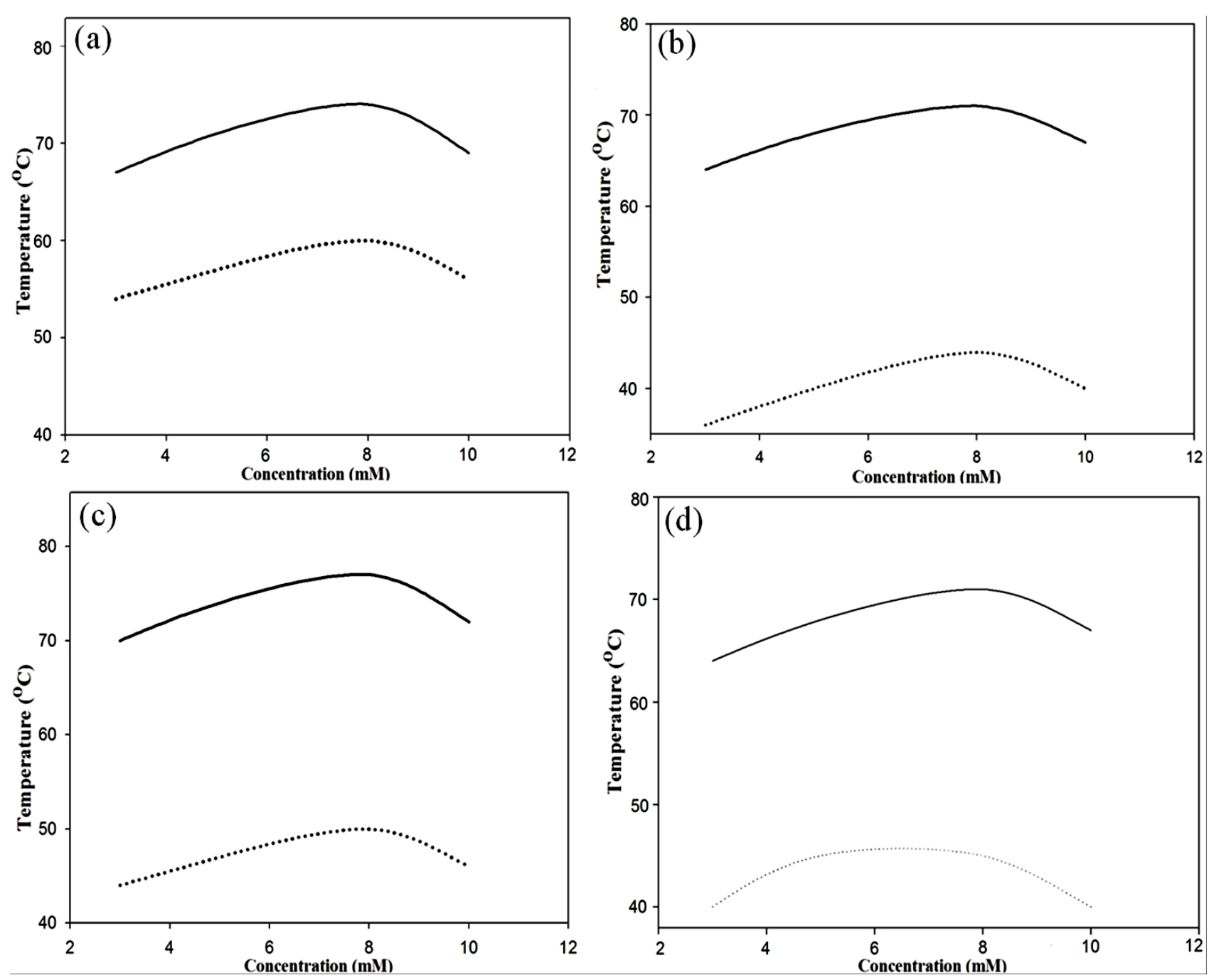

Figure 4.2 The solid curves show the temperatures of onset of turbidity of solutions of Monoand Di-PDMS in hexane and diisopropylamine. Water (5\%) was added at these temperatures. The dashed curves show the temperatures of completion of gelation. (a) Di-PDMS/ diisopropylamine (b) Mono-PDMS/ diisopropylamine (c) Di-PDMS/ hexane (d) Mono-PDMS/ hexane 


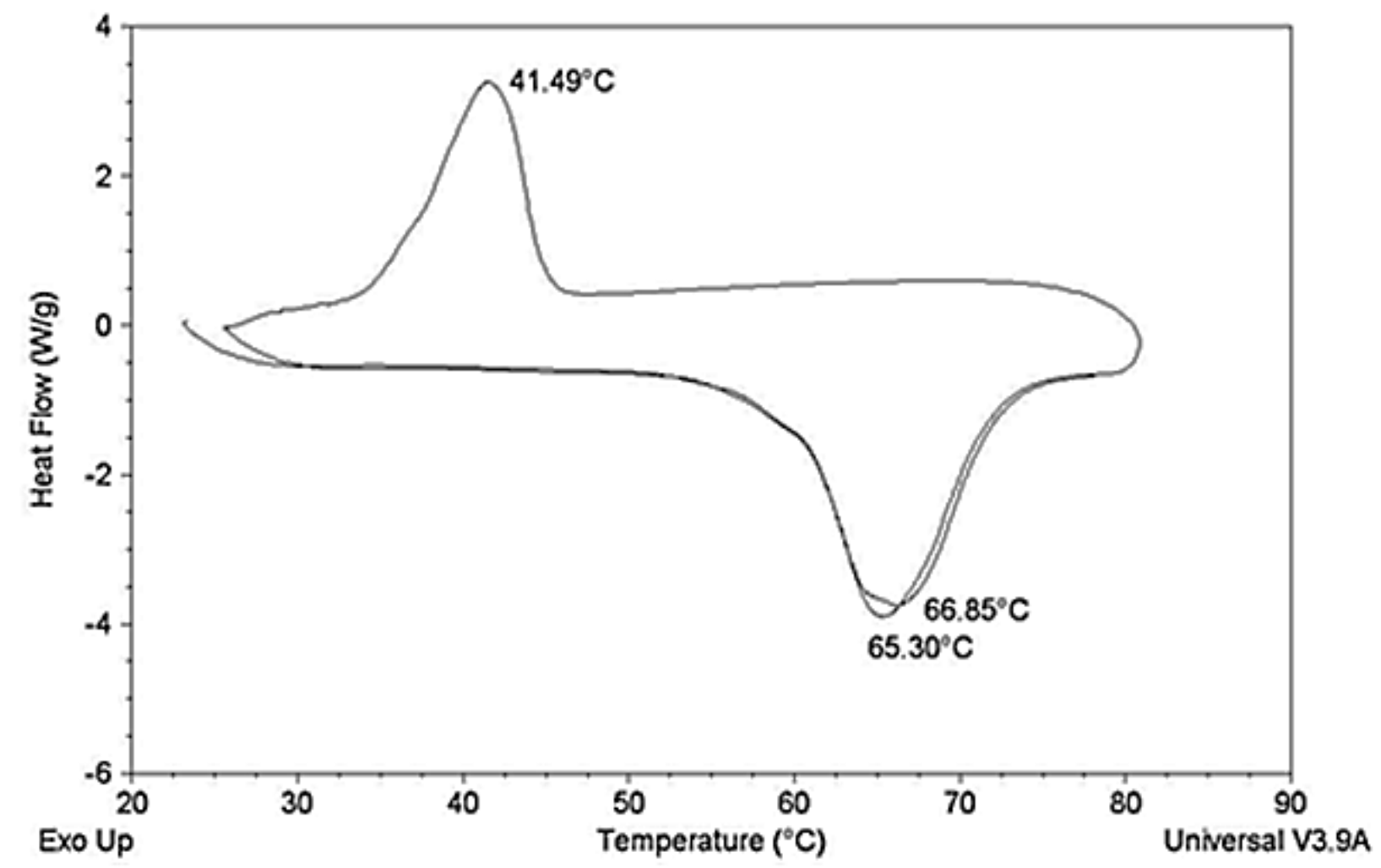

Figure 4.3 DSC curves for the Di-PDMS based gel, during heating, cooling and heating cycles, showing thermo-reversibility of gelation. The sample was kept at $45^{\circ} \mathrm{C}$ during the cooling cycle for 30 minutes before the second heating

\subsubsection{Morphology}

In our previous work ${ }^{10}$, we observed nano-web morphology with drop-cast films of DiPDMS. The polymer, PDMS, by itself will not form a fiber, due to its very low $\mathrm{T}_{\mathrm{g}}$ of $-125^{\circ} \mathrm{C}$. Thus, it is the aggregation of PTCDI, along with intercalation of the PDMS chains that leads to the nano-web morphology that was seen. A schematic of such assembly was given in the previous paper. ${ }^{10}$ The optical microscope images of the propylamine gels with $3 \mathrm{mM}$ and $8 \mathrm{mM}$ concentrations of Di-PDMS are shown in Figures 4.4 a and b respectively. 


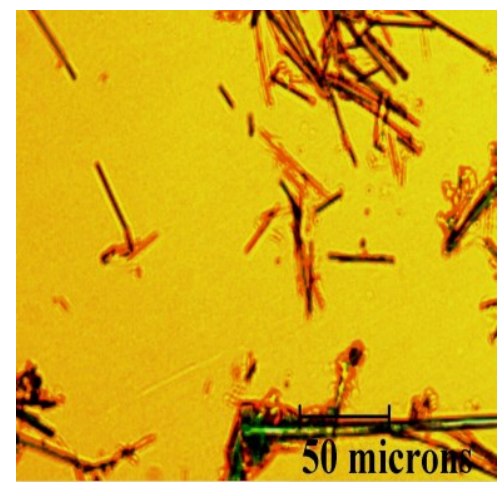

(a)

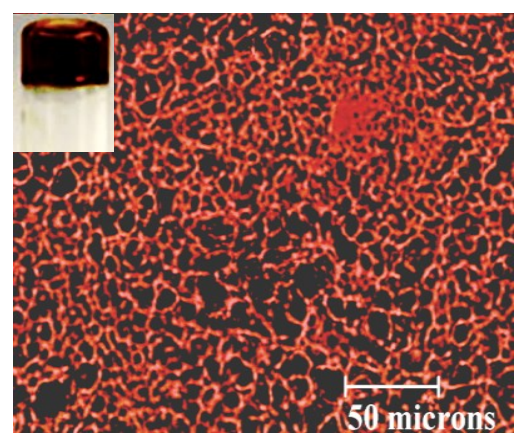

(d)

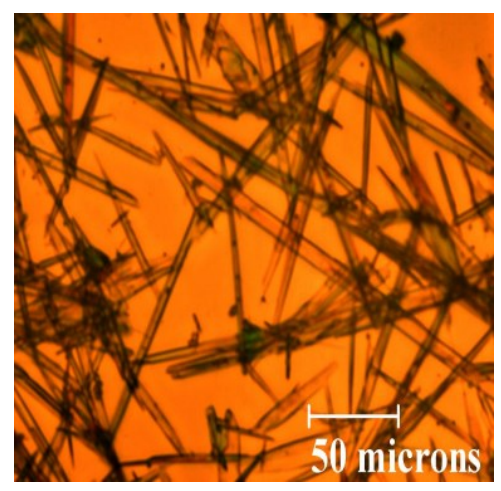

(b)

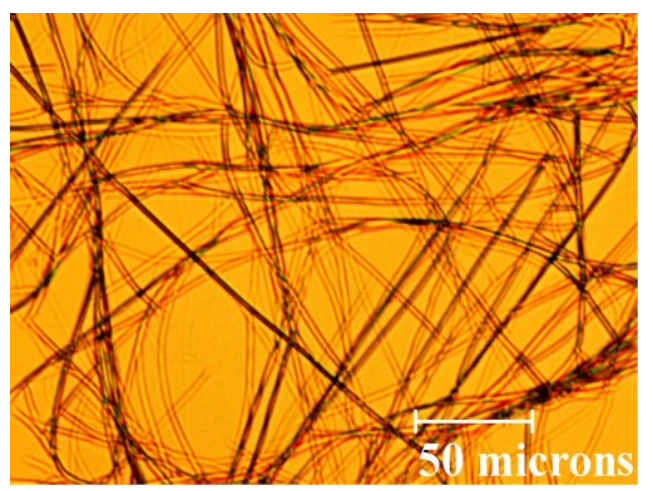

(c)

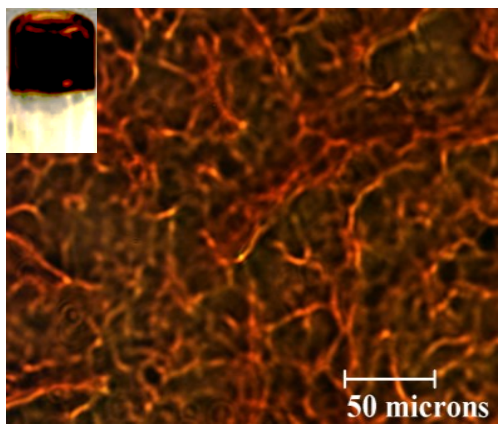

(e)

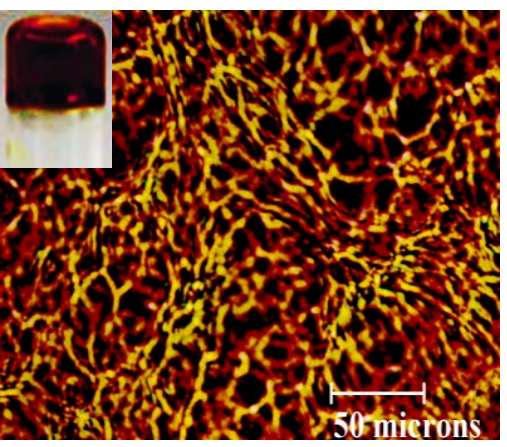

(f)

Figure 4.4 Optical micrographs of Di-PDMS gels with (a), (b), and (c) with propylamine; (d),
(e), and
(f) with hexane/water.
(a) $3 \mathrm{mM}$
(b) $8 \mathrm{mM}$
(c) $8 \mathrm{mM}$ gel after one week. (d) $95 / 5$

(e) $90 / 10$ (f) $85 / 15(8 \mathrm{mM})$. The insets show the inverted vials containing the gels

In both cases, fibrous morphology is seen, with the fibers becoming denser with an increase in concentration. The fibers are a few hundred microns in length. Thus, fibers are formed in the gel, even without the presence of hydrogen bonding groups in the molecule. These fibers continue to grow with time, as seen in Figure 4.4c which was recorded four days after the gel was made. Figures 4.4 d-f show the morphology of the gels made with hexane/water mixtures of $95 / 5,90 / 10$ and $85 / 15$, respectively. The fibers form networks, and the average 
thickness of fibers increased from 2 to $4 \mu \mathrm{m}$ as the water content increases from 5 to $15 \%$. Similar network morphology was seen before in drop-cast films of Di-PDMS from chloroform. ${ }^{10}$ Figure 4.5 shows the SEM micrographs of the xerogels from propylamine and hexane/water.
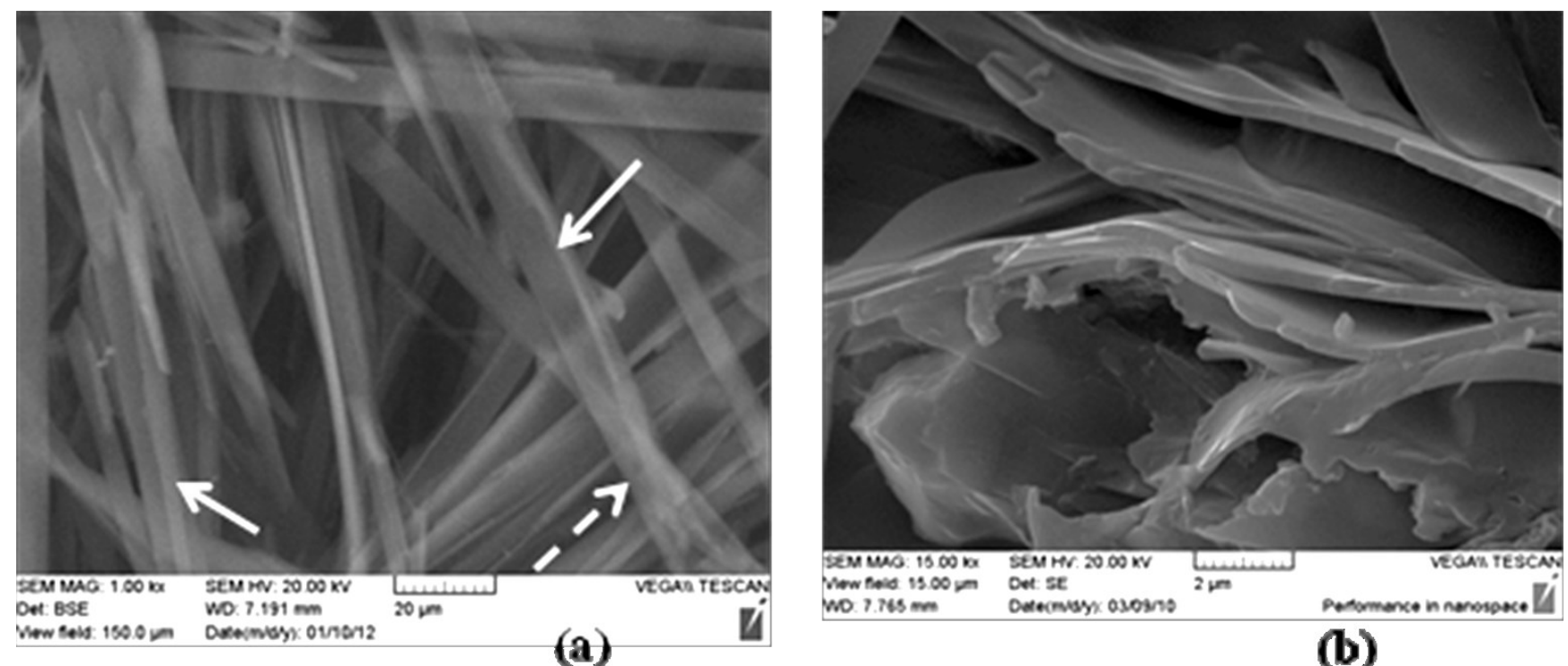

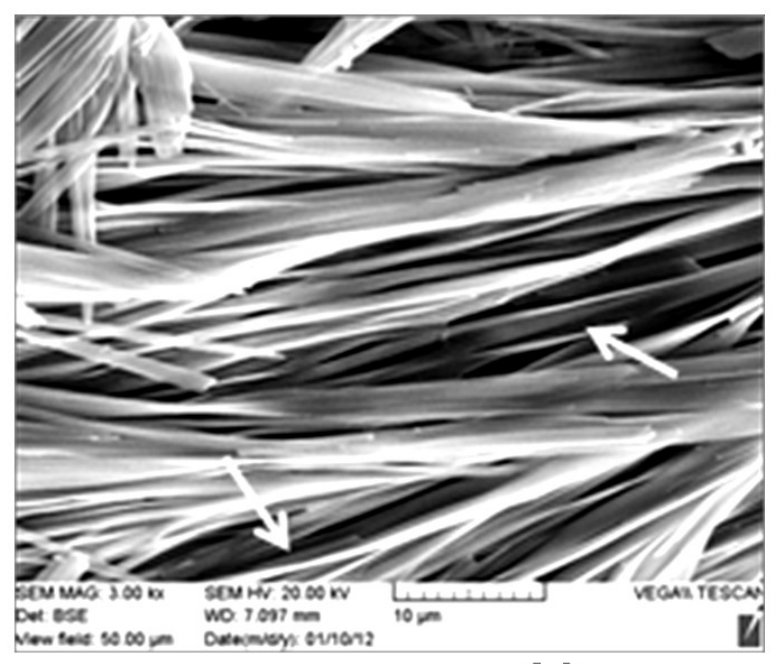

(c) (b)

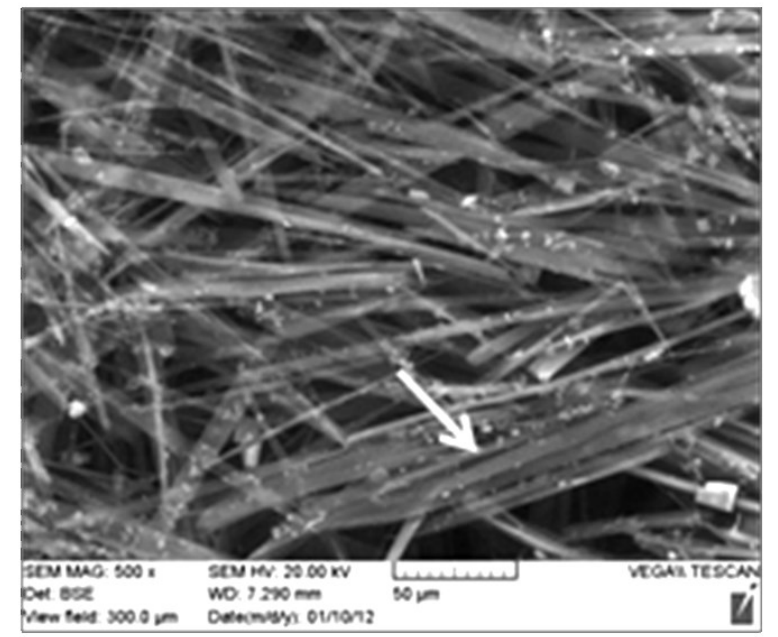

(d)

Figure 4.5 SEM images of (a) Di-PDMS/propylamine xerogel; (b) a cross-section of xerogel in (a); (c) Di-PDMS/hexane/water (8mM/90/10) xerogel; (d) Di-PDMS/hexane/water (8mM/85/15) xerogel 
The fibers of several microns in length are seen in Figure 4.5 a. An image of the crosssection of the gel (cut with a razor blade) is shown in Figure $4.5 \mathrm{~b}$ and consists of sheets with a thickness of about $0.5 \mu \mathrm{m}$. A dense mat of fibers is seen in Figure $4.5 \mathrm{c}$ and $\mathrm{d}$ for the xerogel from hexane/water. An interesting feature seen in Figures $4.5 \mathrm{a}, \mathrm{c}$ and $\mathrm{d}$ is that the fibers are not flat ribbons, but are curled to look like an eaves trough. These are marked with arrows in these figures. These are not perfect hollow tubes. There is some twisting seen as well, as marked with the dashed arrow in Figure 4.5 a. While twisting of ribbons and hollow tube formation is commonly observed with chiral molecules, we have shown before that such hollow tube formation can occur during the assembly of non-chiral molecules due to predominant asymmetry of interactions between molecules in the three directions. ${ }^{30}$ The case that we reported was that of biscarbamate molecules in organogels, in which two hydrogen bonds and van der Waals interactions (between alkyl chains) occur along a sheet of molecules in one direction, and only weak van der Waals interactions in the other two directions. Such sheets do not prefer to be flat, but fold. ${ }^{31-33}$ In the present case, the $\pi$-interaction would occur between the PTCDI units along the packing direction along with van der Waals interactions between the PDMS chains, and weak van der Waals interactions between the aromatic hydrogen atoms and the PDMS segment in the other two directions. Such an asymmetry in interactions seems to be sufficient to cause folding of the ribbons in this case.

Figure 4.6 shows the TEM images of Di-PDMS gels with propylamine and hexane/water (95/5) mixture. The fine structure of the ribbons seen in Figure 4.6 consists of nano-wires, 1$1.2 \mathrm{~nm}$ in thickness. Thus, the presence of the PDMS chain is sufficient to form the nano-wires, without the hydrogen bonding groups in the molecule. 

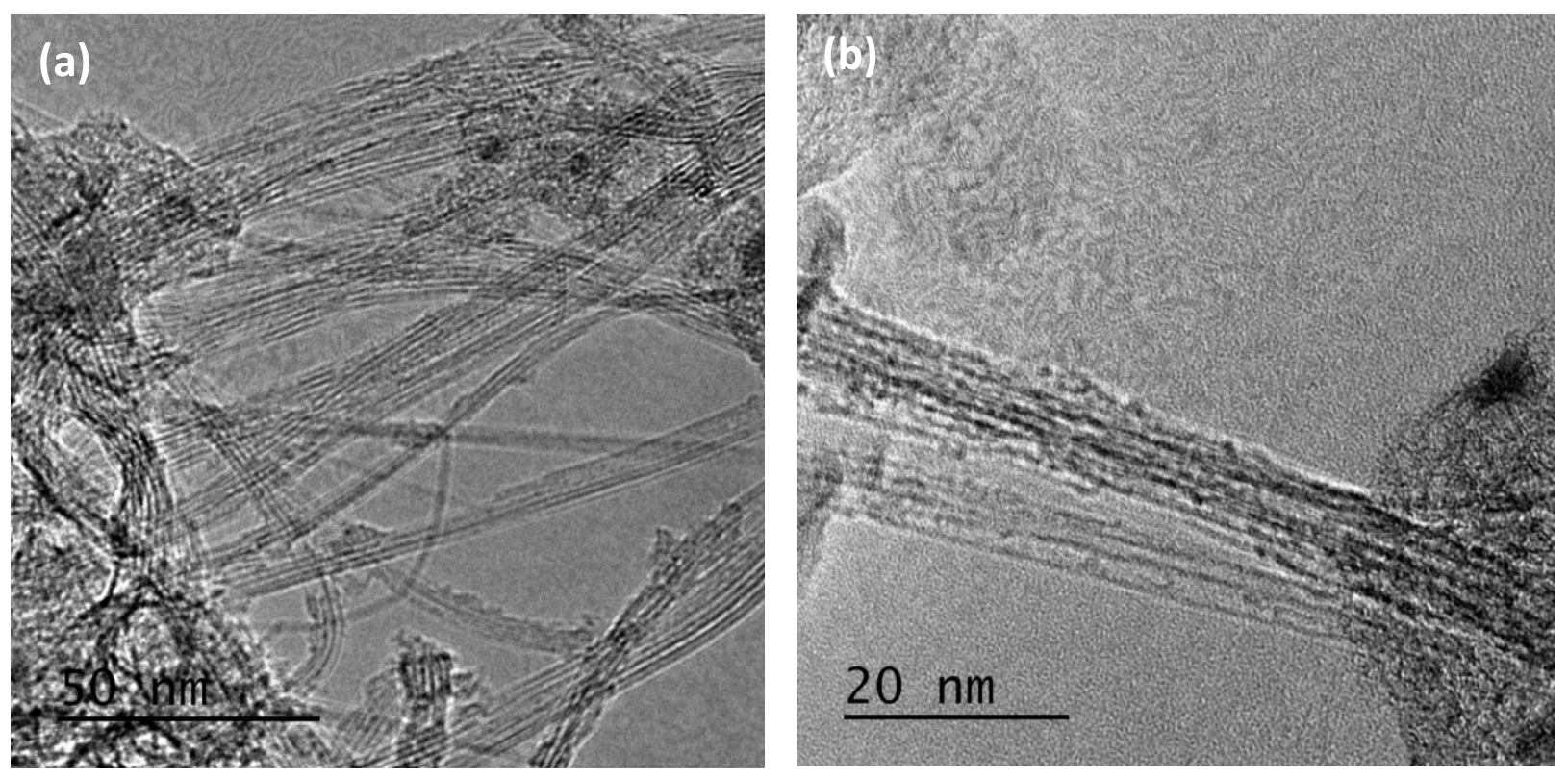

Figure 4.6 Transmission electron microscope images of Di-PDMS gels with (a) propylamine (b) hexane/water: 95/5 mixture

While gelation occurred in the present work without the presence of hydrogen bonding groups, intercalation of PDMS segments is found to be necessary. This is demonstrated by an OM study, as shown in Figure 4.7. Starting from the gel which shows the fibrillar morphology (as in Figure $4.4 \mathrm{~b}$ ), the temperature of the sample was varied in the hot stage of the OM. Melting occurs above $60{ }^{\circ} \mathrm{C}$, and at $100{ }^{\circ} \mathrm{C}$ the sample is completely molten (Figure $4.7 \mathrm{~d}$ ). Upon slowly cooling, the gel fibers form again, as seen in Figure 4.7e. As mentioned above in the discussion of the DSC analysis, the sample was kept at $40{ }^{\circ} \mathrm{C}$ for $30 \mathrm{~min}$. to allow for the gel to develop. Figure $4.7 \mathrm{f}$ shows the completion of gelation. It is worth pointing out that the images in Figures $4.7 \mathrm{a}$ and 4.7 e are very similar. Figure $4.7 \mathrm{~h}$ shows that when the sample was quenched from $100{ }^{\circ} \mathrm{C}$ to room temperature, gel fibers did not form. Instead, crystals of PTCDI are seen. The same behavior was seen with mono-PDMS as well, as shown in Figure 4.8. This would indicate that the PDMS segments did not intercalate to form the gel fibers upon quenching. 

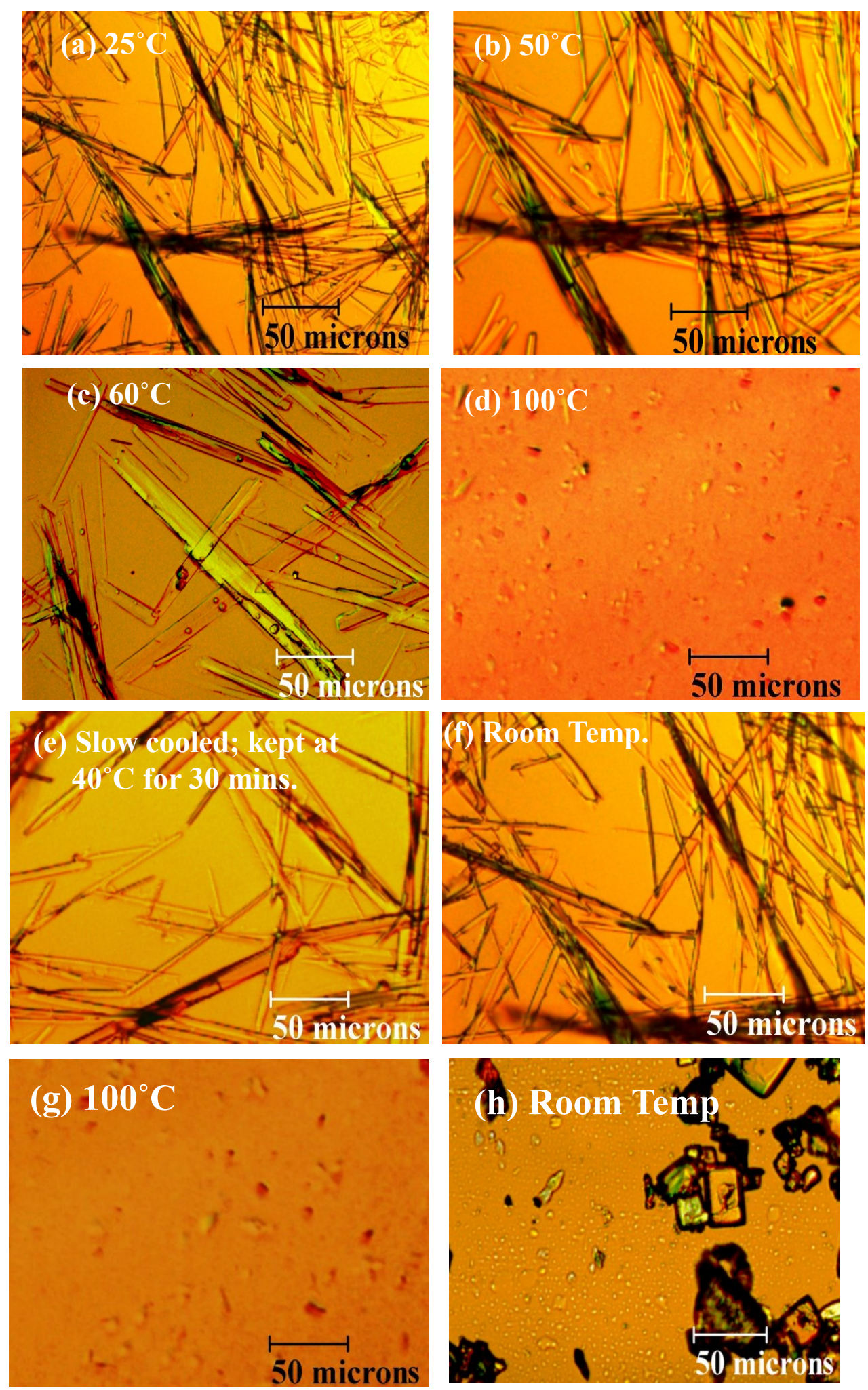

Figure 4.7 (a) - (f): Thermo-reversibility of the Di-PDMS gel by slow-cooling; (g) and (h): Irreversibility by quenching 

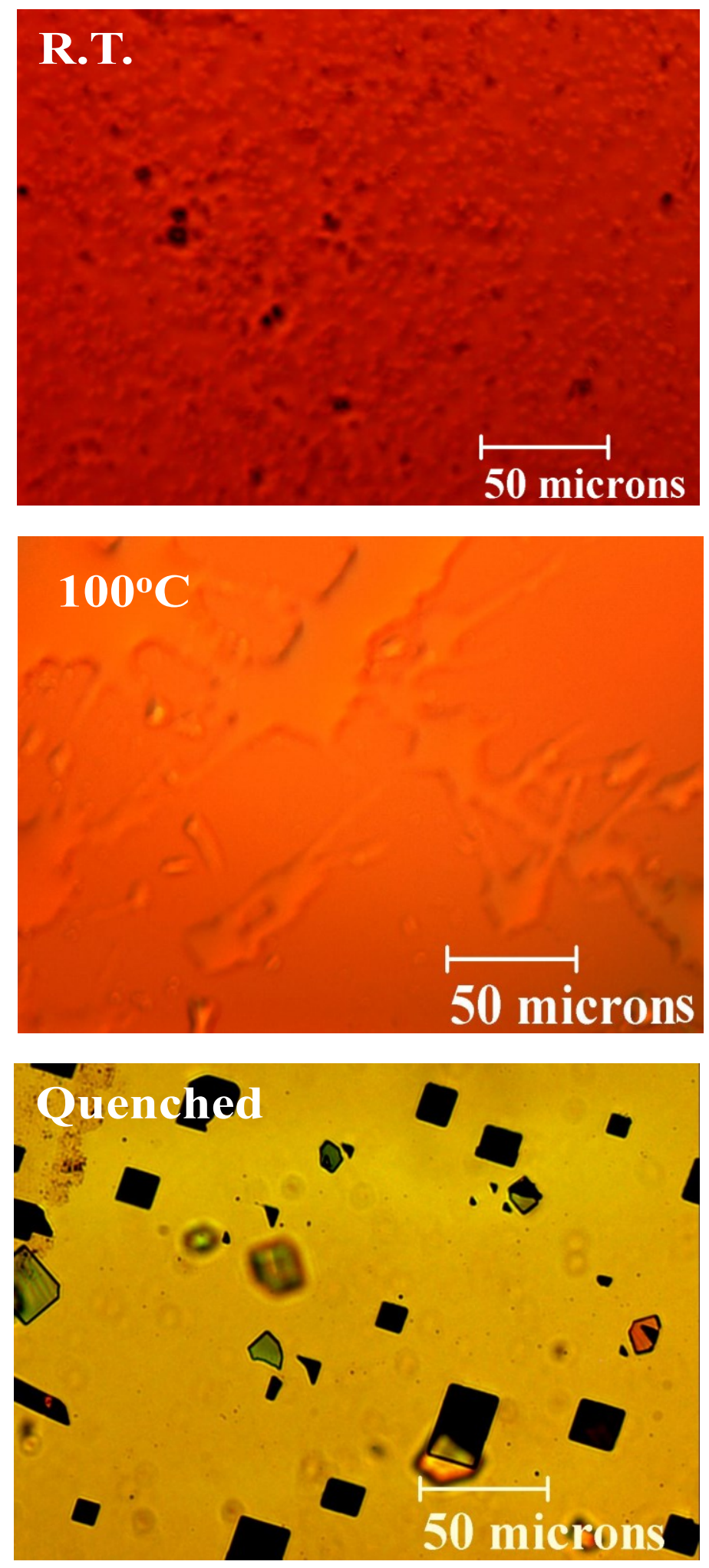

Figure 4.8 The Mono-PDMS gel is not reversible if the sample is quenched from the hot solution. Crystals are formed instead. Slow cooling leads to thermo-reversible gel 
Slow cooling is necessary for gelation to occur. We had observed gelation with small molecule gelators as well as polymers upon slow cooling as well as quenching, although the resulting morphology would differ. However, in the present case, slow cooling is required to enable intercalation of the PDMS segments. It is realized that the entanglement molecular weight $\mathrm{M}_{\mathrm{c}}$ and molecular weight between entanglements $\left(\mathrm{M}_{\mathrm{e}}\right)$ for PDMS are 24,500 and 12,000, respectively. ${ }^{34,35}$ These numbers correspond to the behavior of the polymer in the dense melt, whereas we are dealing with solution here, and with the PDMS segment anchored to PTCDI.
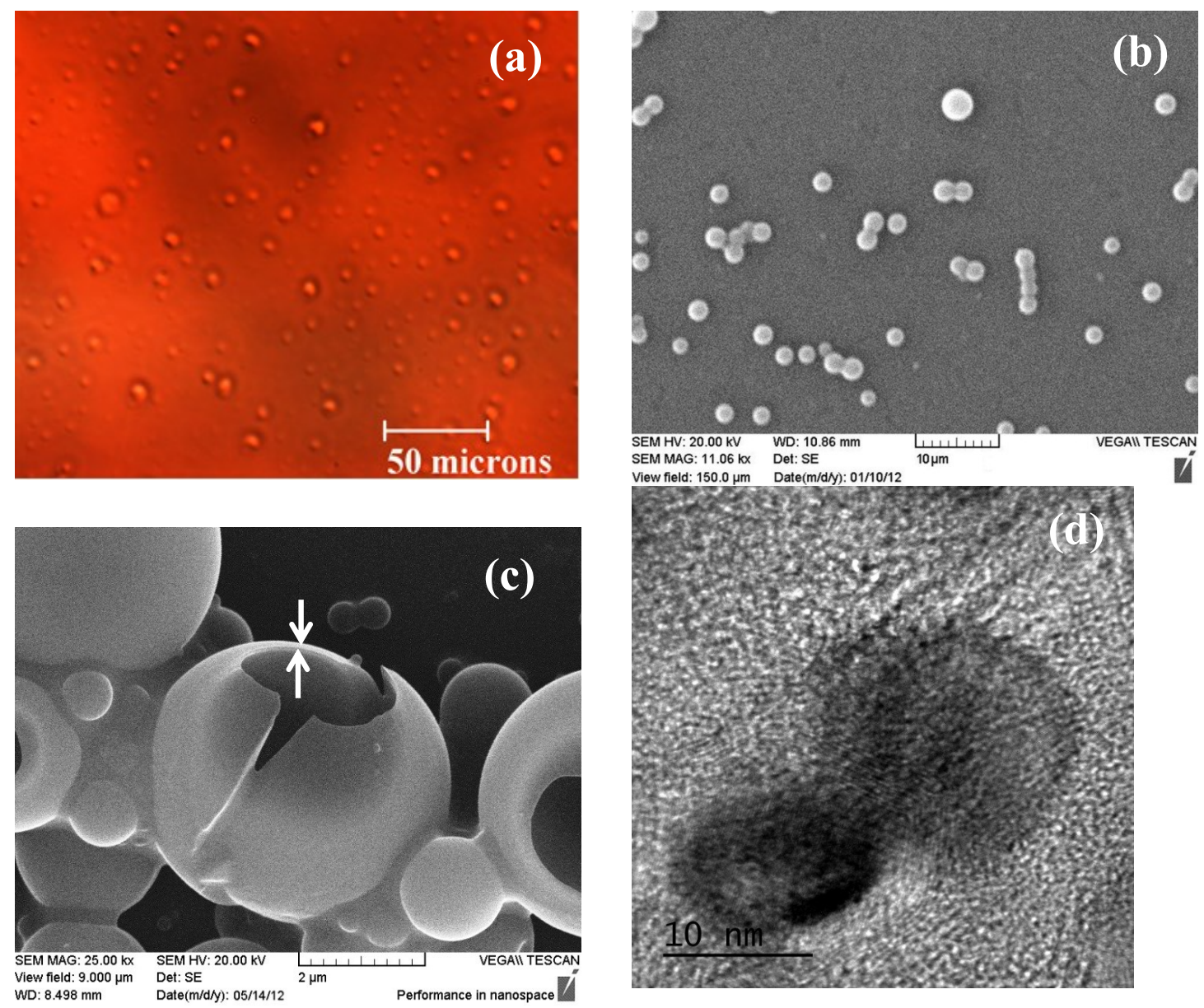

Figure 4.9 (a) Optical microscope image of Mono-PDMS/propylamine gel (b) SEM of the xerogel (c) SEM of fractured gel (d) TEM image of the xerogel 
As mentioned above, the organogels of mono-substituted PDI have not been reported so far. Figure $4.9 \mathrm{a}$ and $\mathrm{b}$ show the optical and scanning electron micrographs, respectively, of the Mono-PDMS gel, which consist of spherical structures of 2-4 $\mu \mathrm{m}$ in diameter. Our previous study on drop-cast films showed vesicular morphology. ${ }^{9}$ To verify if the spherical structures are hollow, a piece of the gel was kept between glass slides, dipped in liquid nitrogen and a slight pressure was applied. The SEM image in Figure 4.9 c shows the fracture morphology, and confirms that the spheres are hollow. The thickness of the wall, measured as shown by the arrows, is about $50 \mathrm{~nm}$. The TEM image in Figure $4.9 \mathrm{~d}$ shows even smaller spheres of about 10 $\mathrm{nm}$ in diameter.

Both Mono- and Di-PDMS gel propylamine, and the transition temperatures are nearly the same (as seen from DSC analysis). We examined if in a blend of these the perylene segment of these two molecules would overlap and form a molecular complex during gelation or they would self-sort. We find that each of the components maintained their inherent morphologies during gelation. Figures 4.10 a-c show the OM images of gels of 80/20, 50/50 and 20/80 Mono/Di-PDMS. The spherical morphology due to Mono-PDMS is seen along with the fibrous growth of Di-PDMS on these spherical domains. These two morphologies are not isolated from each other. With Di-PDMS as the minor component, short fibers are seen in Figure 4.10 a. The thickness of the fibers increases with the Di-PDMS content. The SEM image in Figure $4.10 \mathrm{~d}$ for $80 \%$ Mono-PDMS shows that its spherical morphology is distinct, with fibers of Di-PDMS embedded in it. With 50/50 mixture of the two, fibers of Di-PDMS are seen (Figure 4.10 e), with the spheres of Mono-PDMS as over-growth. Note that most of these spheres are adhering to the fibers, as if they coalesce and assemble directionally along the fibers. With Di-PDMS as the 
major component, the fibers become denser. The thickness of the fibers increases from about 115 $\mathrm{nm}$ in Figure 4.10 e to $1-2 \mu \mathrm{m}$ in Figure $4.10 \mathrm{f}$ for $80 \%$ Di-PDMS.
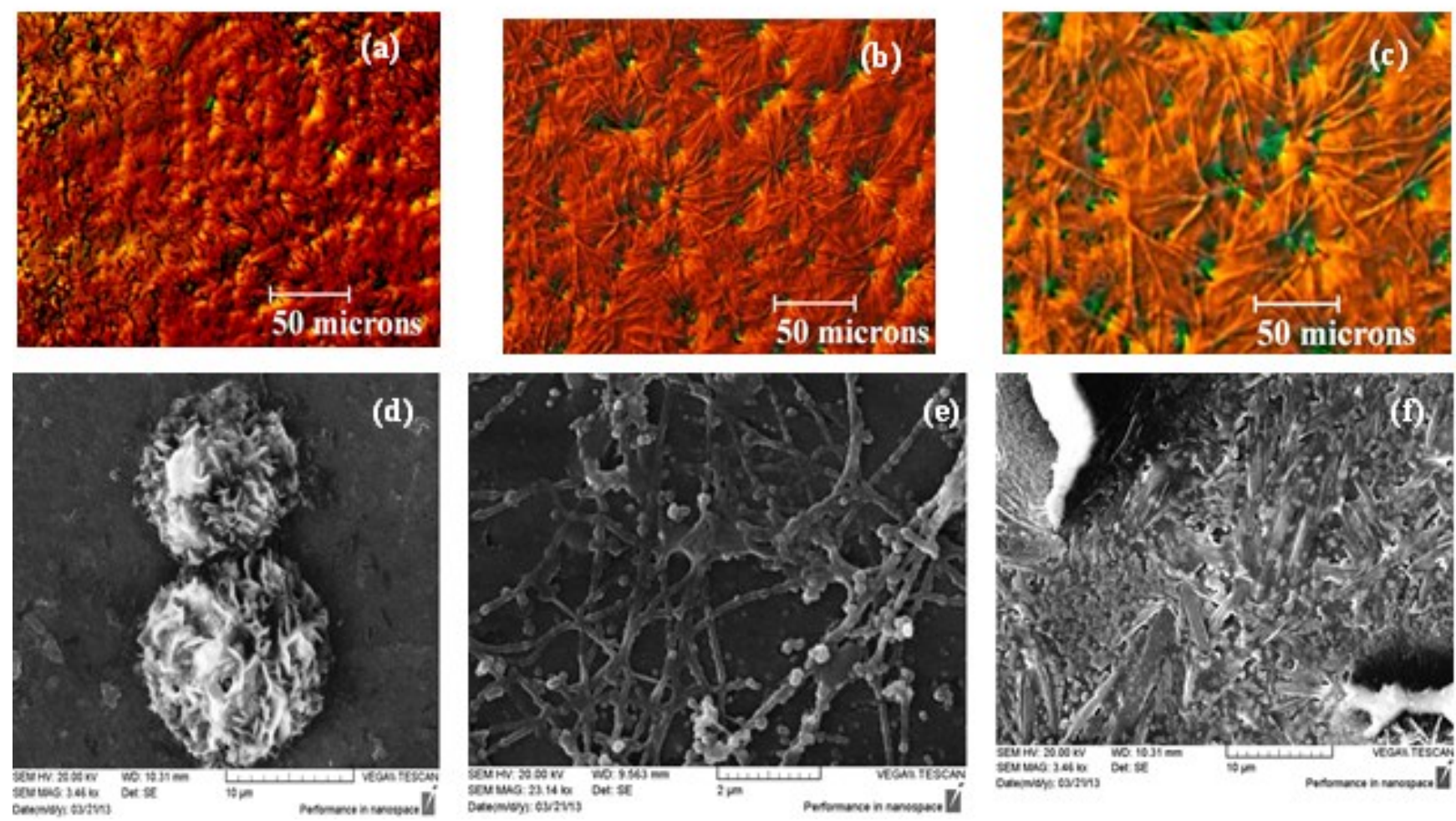

Figure 4.10 (a), (b) and (c): OM of gels with 80/20, 50/50 and 20/80 Mono- / Di-PDMS, respectively; (d), (e) and (f): SEM images of gels with 80/20, 50/50 and 20/80 Mono-/ DiPDMS, respectively

X-ray diffraction (XRD) confirmed the crystalline nature of the gels due to the stacking of the perylene segment. Figure 4.11 shows the XRD from the xerogels of Mono- and DiPDMS. The $\mathrm{M}_{\mathrm{w}}$ of the PDMS was 1500 in these cases. Since the (amorphous) PDMS concentration in Di-PDMS is twice that in Mono-PDMS, the crystalline peaks are not well resolved for the former in Figure 4.11 (b). The effect of the PDMS on the diffraction can be seen in Figure 4.12, which shows the XRD from drop-cast films of Mono- and Di-PDMS, with PDMS 
molecular weights of 900 and 3000. The number of peaks becomes reduced for Mono-PDMS as the $\mathrm{M}_{\mathrm{w}}$ of PDMS increases from 900 to 3000 . Only two broad peaks are seen for Di-PDMS with $\mathrm{M}_{\mathrm{w}}=900$ and just one with $\mathrm{M}_{\mathrm{w}}=3000$. Thus the crystalline diffraction due to the stacking of the perylene segment is masked by the diffraction from PDMS, especially since Si is a heavier atom. 

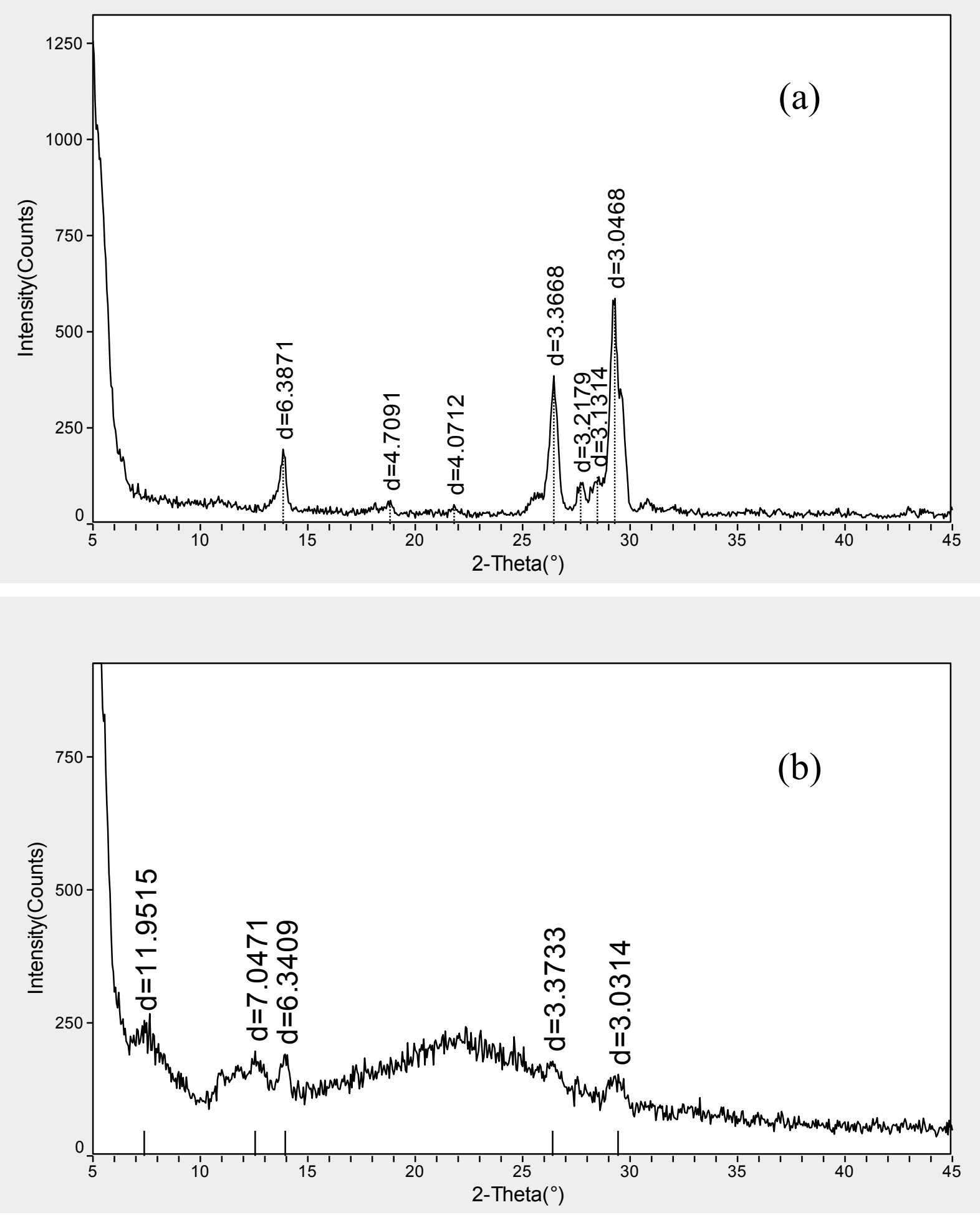

Figure 4.11 X-Ray diffraction traces from the xerogels of (a) Mono-PDMS and (b) Di-PDMS. The PDMS $\mathrm{M}_{\mathrm{w}}=1500$ 
In the case of self-assembled systems, one would expect the packing to be similar in the crystalline state and in the gels. However, we find differences between the powder XRD of as synthesized samples and the xerogels. With Mono-PDMS, our previous work ${ }^{9}$ showed XRD of the as-synthesized powder sample with d-spacings of $7.7(\mathrm{~s}), 6.34(\mathrm{w}), 3.61(\mathrm{~m}), 3.35(\mathrm{~m})$ and 3.01(w) A. (The intensities are qualitatively stated as strong, medium, and weak). With the XRD of the xerogel of Mono-PDMS (Figure 4.11 (a)), the $7.7 \AA$ reflection is absent. Those corresponding to 3.36 and 3.04 are strong, and the $6.39 \AA$ reflection is of medium intensity. With Di-PDMS, the XRD of the as-synthesized sample showed only broad reflections with spacings of 7.52 and $3.51 \AA$. We find the XRD of the Di-PDMS xerogel to be different, as seen in Figure 4.11 (b). Apart from noting these differences, it would be awkward to draw any conclusions regarding the crystalline packing, with just two or three reflections.
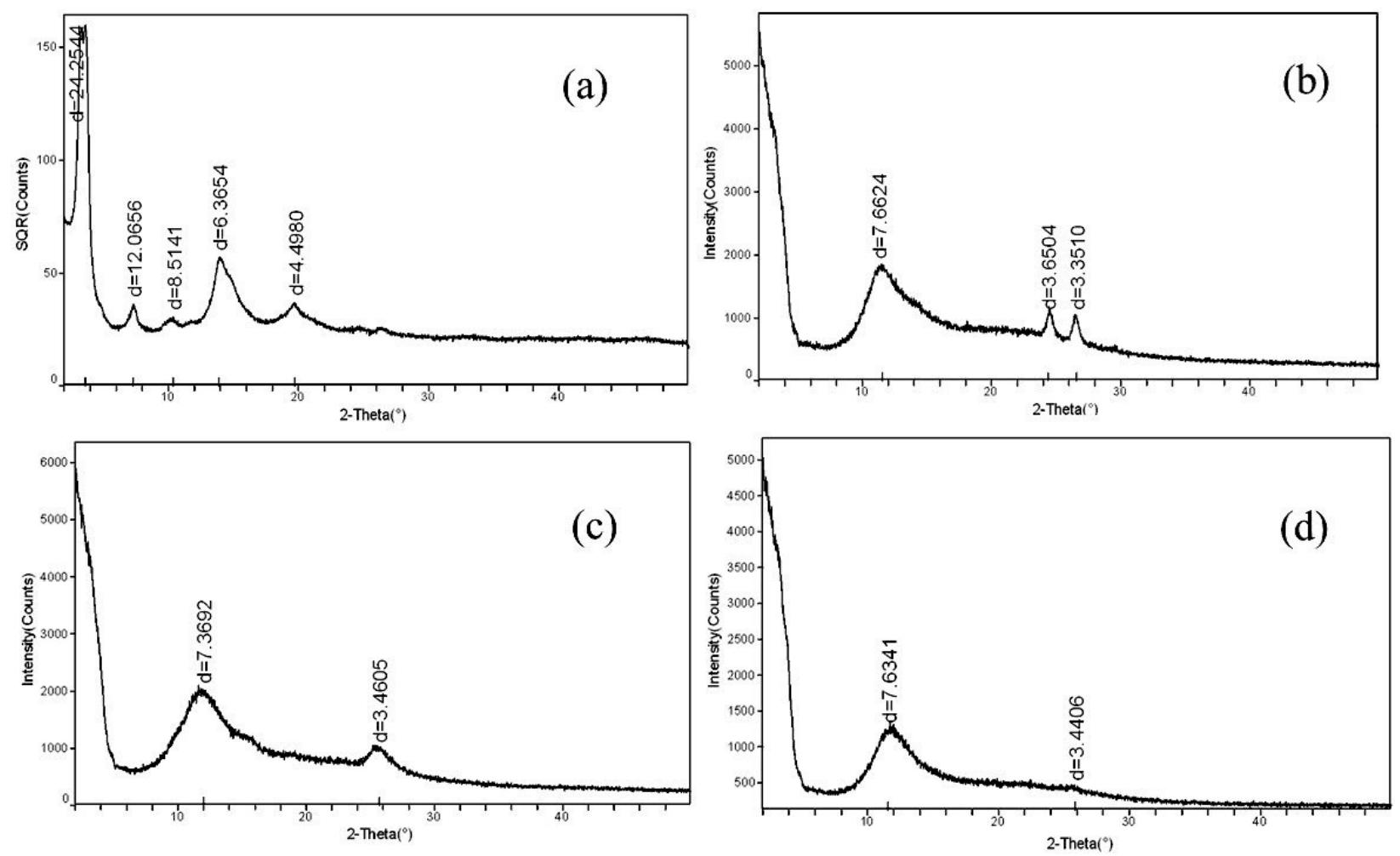

Figure 4.12 X-ray diffraction profile of films of (a) and (b) Mono-PDMS; (c) and (d): DiPDMS. (a), (c) with $M_{w}$ of 900; (b), (d) $M_{w}$ of 3000 for the PDMS segment 


\subsubsection{Optical Properties}

Figure 4.13 shows the UV-Vis absorption and fluorescent spectra of the Mono- and DiPDMS gels as a function of concentration. Also included in Figures 4.13 (a) and 4.13 (c) are the absorption spectra in chloroform solution, with $10 \mathrm{mM}$ concentration. With Mono-PDMS, the chloroform solution shows a shoulder at $441 \mathrm{~nm}$, and maxima at 515 and $560 \mathrm{~nm}$, with the 515 $\mathrm{nm}$ peak being more intense. The absorption spectra of the gels (Figure 4.13 (a)) show peaks at 472, 496 and a shoulder at $546 \mathrm{~nm}$. No significant shift is seen with concentration. With $3.0 \mathrm{mM}$ concentration, $\mathrm{I}_{472}$ is slightly lower than $\mathrm{I}_{496}(0.9 / 1.0)$, and these peaks are of equal intensity with $5 \mathrm{mM}$ concentration. With further increase in concentration, the $\mathrm{I}_{472}$ peak is the most intense. The decrease of fluorescent intensity with concentration as seen in Figure 4.13 (b), combined with the blue shift of the maxima from the solution to the gel state indicates H-stacking of the perylene units in the gel. With Di-PDMS/chloroform solution the maxima are seen at 538 and $600 \mathrm{~nm}$ and they blue-shift to 498 and $540 \mathrm{~nm}$ in the case of the gels $(10 \mathrm{mM})$.

A small blue shift is seen as the concentration is increased from $3 \mathrm{mM}(503,547 \mathrm{~nm})$ to $10 \mathrm{mM}(498,540 \mathrm{~nm})$. A shoulder is seen at $620 \mathrm{~nm}$. Figure 4.13 (d) shows that the fluorescent intensity decreases with an increase in concentration of Di-PDMS in the gels. This indicates that the perylene units are H-stacked in the Di-PDMS gels also. The absorption spectra of the xerogels are shown in Figure 4.14. In contrast to the case of the gels where there was no significant change in the peak positions with concentration, a red shift is seen in the case of xerogels. With Mono-PDMS xerogel, as the concentration increases from 1.0 to $10.0 \mathrm{mM}$, the $\mathrm{S}_{0 \text { - }}$ 1 peak shifts from 492 to $502 \mathrm{~nm}$, and the $\mathrm{S}_{0-0}$ peak, from 548 to $556 \mathrm{~nm}$. The red-shift is more for the xerogels of Di-PDMS, with the $\mathrm{S}_{0-1}$ peak shifting from 507 to $525 \mathrm{~nm}$ and the $\mathrm{S}_{0-0}$ peak 
from 582 to $604 \mathrm{~nm}$. Hence, a reorganization seems to take place in the stacking of the perylene units during the drying process.
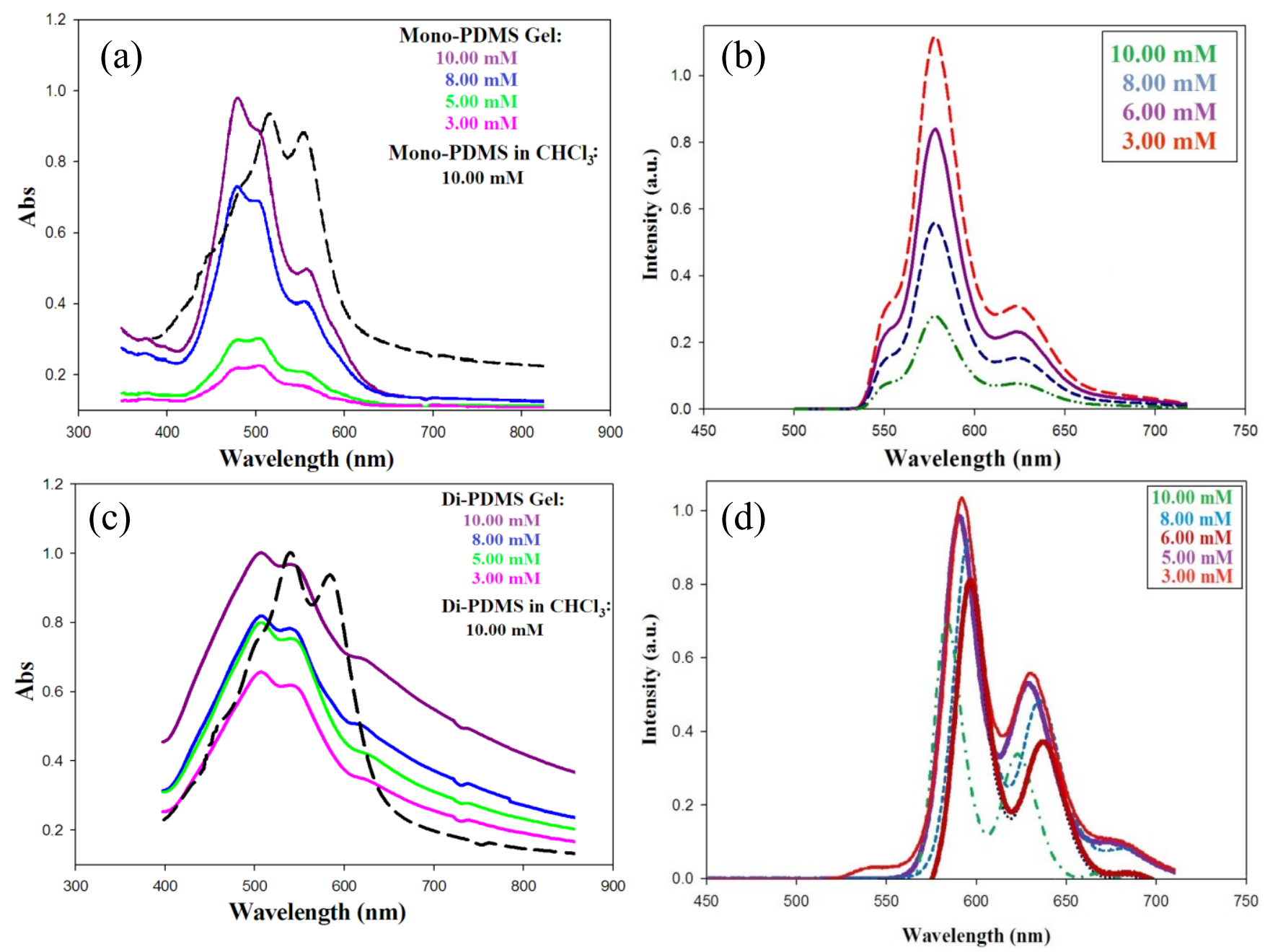

Figure 4.13 (a) UV-Vis absorption spectra of Mono-PDMS/propylamine gel with various concentrations of Mono-PDMS. A solution spectrum in chloroform (10 mM) is also shown. (b) Fluorescence spectra of Mono-PDMS gel with various concentrations (c) UV-Vis absorption spectra of Di-PDMS/propylamine gel with various concentrations of Di-PDMS. A solution spectrum in chloroform $(10 \mathrm{mM})$ is also shown. (d) Fluorescence spectra of Di-PDMS gel with various concentrations 

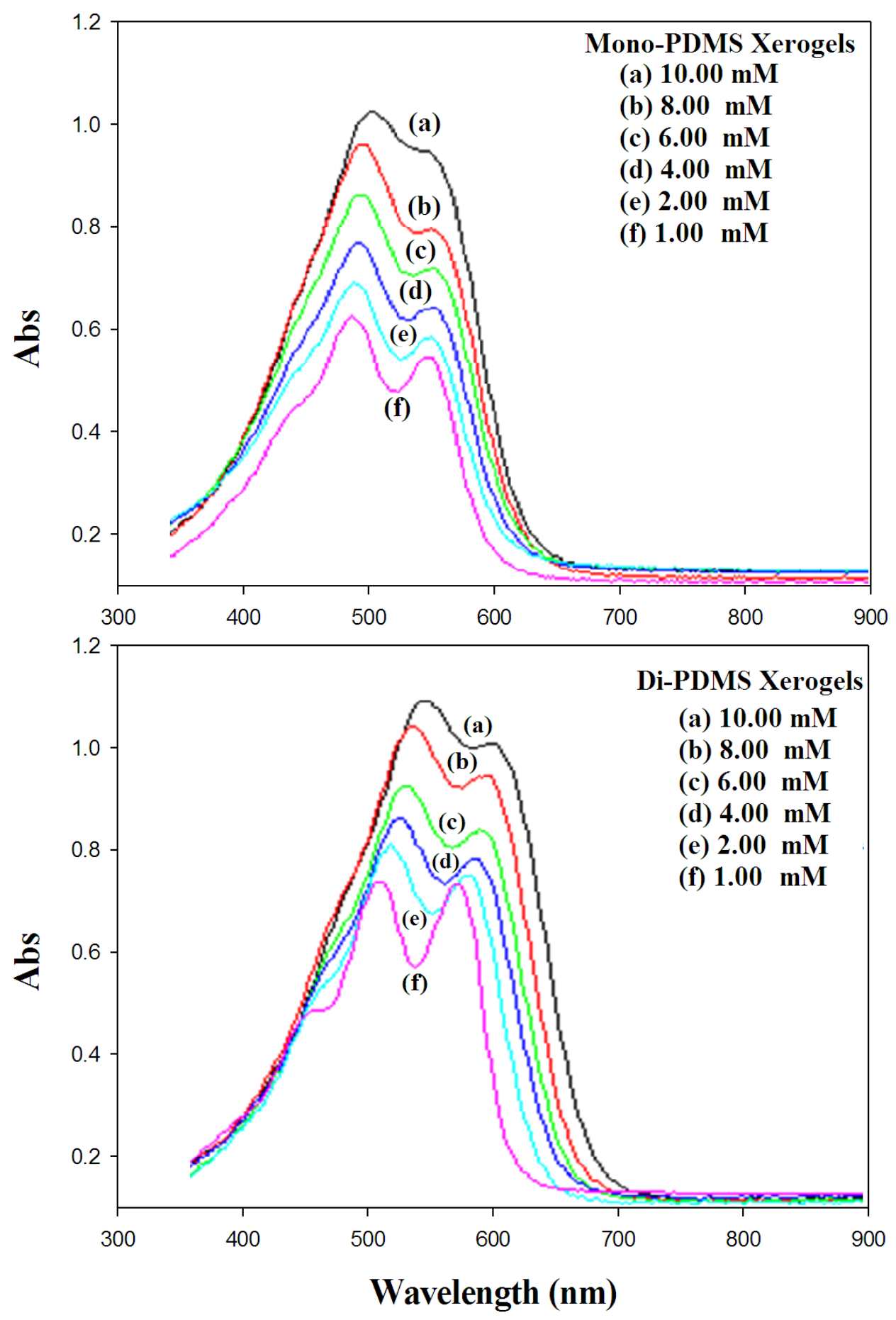

Figure 4.14 Absorption spectra of xerogels of Mono- and Di-PDMS 
The absorption spectra for gels $(10 \mathrm{mM})$ with blends of Di- and Mono-PDMS with 80/20, 20/80 and 50/50 ratios are shown in Figure 4.15. Also shown are the spectra in choloroform solutions. The solution spectra of Di- and Mono- PDMS (Figure 4.13) showed the presence of self-assembled structure of the perylene segment. However, the solution spectra in Figure 4.15 for the blends show only monomeric structure. With Mono-/Di- PDMS ratios of 80/20 and 50/50 in solution, Figures 4.15 (a) and (c) show that the 0-1 and 0-0 peaks are at 530 and $575 \mathrm{~nm}$ respectively, in-between those of 100\% Mono- and 100\% Di- PDMS solutions in Figures 4.13 (a) and (c). A well-defined 0-2 peak is also seen at $495 \mathrm{~nm}$. However, with Mono-/Di- PDMS ratio of 20/80, a significant red shift is seen, with peaks appearing at 570 and $610 \mathrm{~nm}$. As for the gels of the $80 / 20$ and 50/50 blends, the $0-1$ and $0-0$ peaks are seen at 465 and 502, respectively, which are similar to those for 100\% Mono-PDMS gel (473 and 498nm, Figure 4.13 a). When DiPDMS is the predominant component (Mono-/Di- PDMS: 20/80), a large red shift is seen with peaks at 536 and $582 \mathrm{~nm}$ (compared to the peaks at 498 and $540 \mathrm{~nm}$ for the 100\% Di-PDMS gel). Thus, the presence of Mono-PDMS as a minor component significantly affects the absorption spectrum of the blend. 

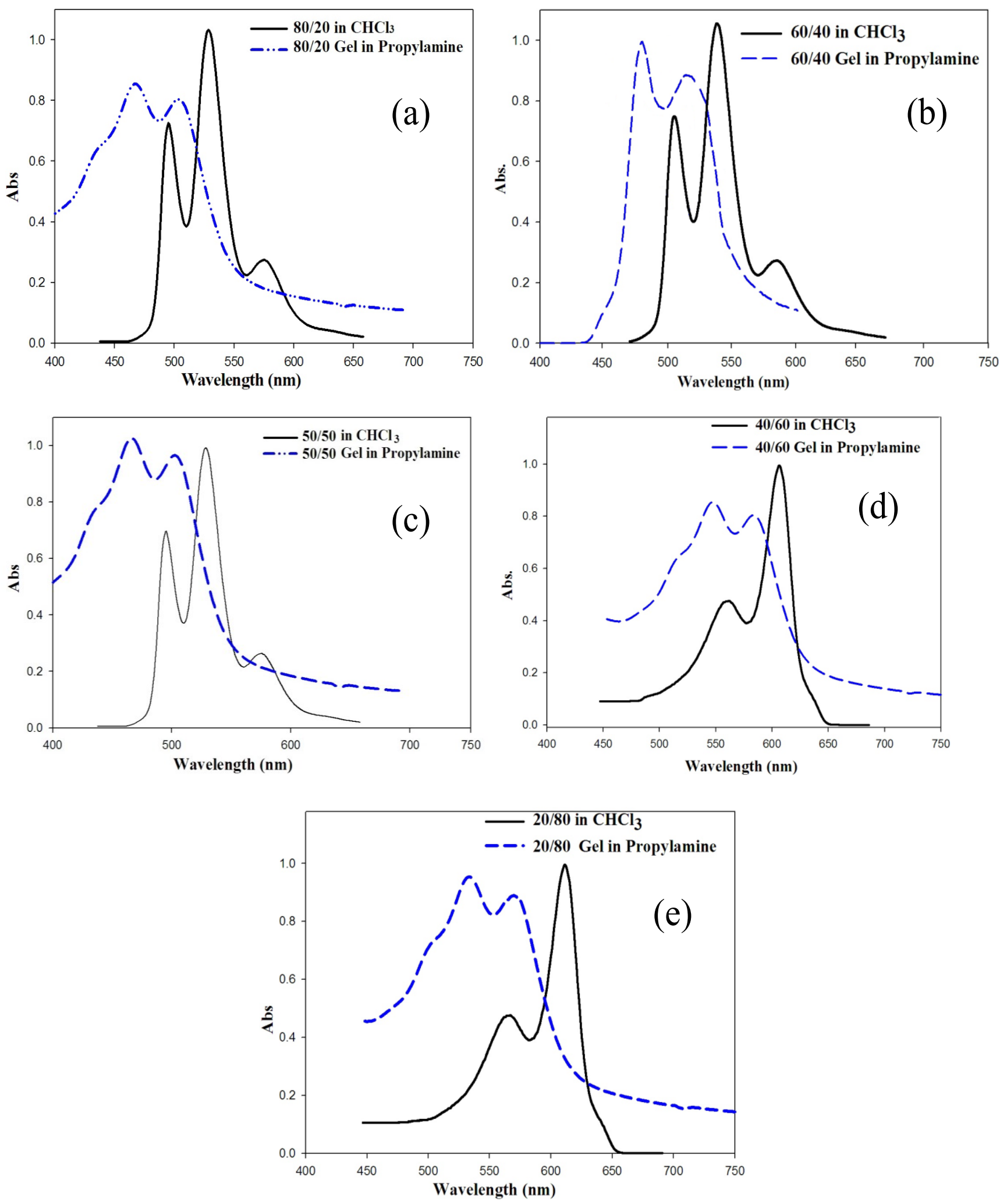

Figure 4.15 Absorption spectra of gels and solutions of blends of Mono- and Di-PDMS. The weight ratios of Mono/ di-PDMS are shown 
The absorption spectra for the gels of Mono- and Di-PDMS prepared with various concentrations in hexane/water mixtures (95/5) are shown in Figures 4.16 (a) and (c), respectively. The peaks are well resolved compared to those in Figure 4.13 for the gels prepared with propylamine. The 0-1 peak for Mono-PDMS shifts from 547 to $550 \mathrm{~nm}$ with an increase in concentration. The red shift of the $0-0$ peak is more significant, from 580 to $596 \mathrm{~nm}$. Likewise, for Di-PDMS, the 0-1 peak shifts from 532 to $540 \mathrm{~nm}$, and the 0-0 peak, from 578 to $588 \mathrm{~nm}$. Figures 4.16 (b) and (d) show that with an increase in the concentration of water to $15 \%$, the spectrum changes drastically. For Mono-PDMS (Figure 4.16 (b)), when the water content is increased from 5 to $15 \%$, the peak at $550 \mathrm{~nm}$ decreases in intensity, the peak at $596 \mathrm{~nm}$ blueshifts to $582 \mathrm{~nm}$. The ratio of the intensities of the two peaks increases from 0.48 to 0.92 . In addition a shoulder appears at $515 \mathrm{~nm}$. For Di-PDMS, (Figure 4.16(d)), the 0-0 peak at $588 \mathrm{~nm}$ shifts to $561 \mathrm{~nm}$ and there is an intensity reversal between the $0-1$ and $0-0$ peaks. This shows that while addition of a small amount of water to hot hexane solution leads to gelation, higher amounts lead to gelation and crystallization. This is also seen in Figure 4.5 (d) which shows precipitated crystals along with gel fibers with hexane/water: 85/15. 

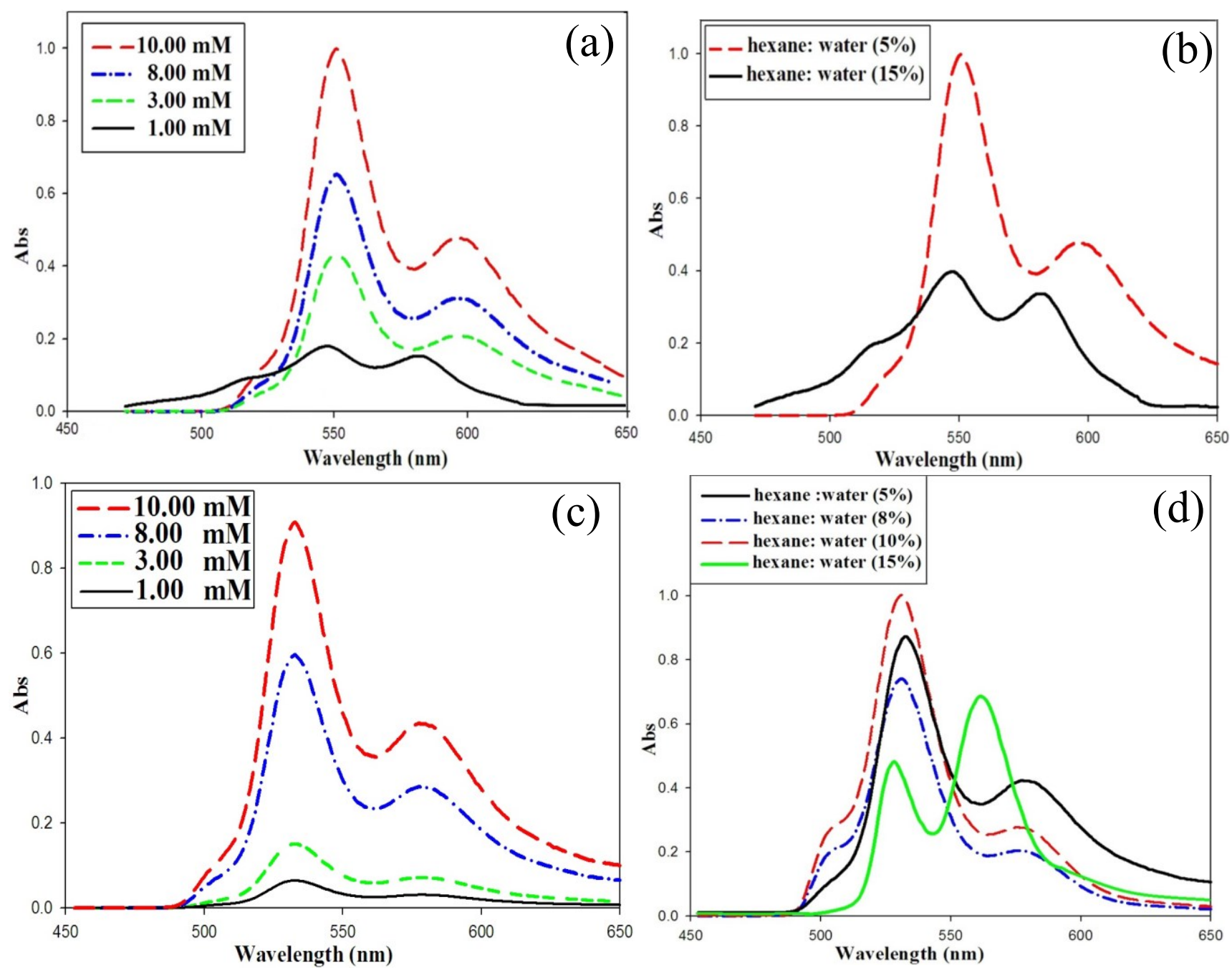

Figure 4.16 Absorption spectra of (a) Mono-PDMS gels in hexane/ water: 95/5 mixtures, with different concentrations. (b) Mono-PDMS gels (10mM) in hexane/water: 95/5 and 85/15. (c) DiPDMS gels in hexane/ water: 95/5 mixtures, with different concentrations. (d) Di-PDMS gels $(10 \mathrm{mM})$ in hexane/ water mixtures with different water concentrations

To estimate the extent of precipitated crystals, the heat of fusion $(\Delta \mathrm{H})$ was measured for the xerogels with various Di-PDMS concentrations, for hexane/water: $95 / 5$ and $85 / 15$ compositions. Table 4.1 shows that $\Delta \mathrm{H}$ is consistently higher with $15 \%$ water concentration. It is also seen that $\Delta \mathrm{H}$ is very large with the $3.0 \mathrm{mM}$ concentration of Di-PDMS. This is due to the 
fact that this is the minimum concentration required for gelation, and while some of the material causes gelation, the rest precipitate.

Table 4.1. Variation of heat of fusion of perylene crystals in xerogels of Di-PDMS with hexane/water mixtures

\begin{tabular}{|c|c|c|}
\hline Di-PDMS Xerogel (mM) & \multicolumn{2}{|c|}{ Heat of Fusion (J/g) } \\
\hline & Hexane: Water (5\%) & Hexane: Water (15\%) \\
\hline 3.00 & 13.02 & 23.52 \\
\hline 6.00 & 0.806 & 6.770 \\
\hline 8.00 & 0.813 & 10.97 \\
\hline 10.00 & 0.923 & 15.17 \\
\hline
\end{tabular}

The quantum yields $(\Phi)$ for the gels were calculated using Rhodamine $6 \mathrm{G}$ as the standard. Figure 4.17 shows the variation of $\Phi$ with concentration for Mono- and Di-PDMS gels with propylamine and hexane/ water: $95 / 5$ mixtures. It is less than 0.7 for all cases, with the gels in mixed solvents showing higher $\Phi$. The propylamine based gels show very little variation with concentration, whereas the change is more significant in the mixed solvent. 

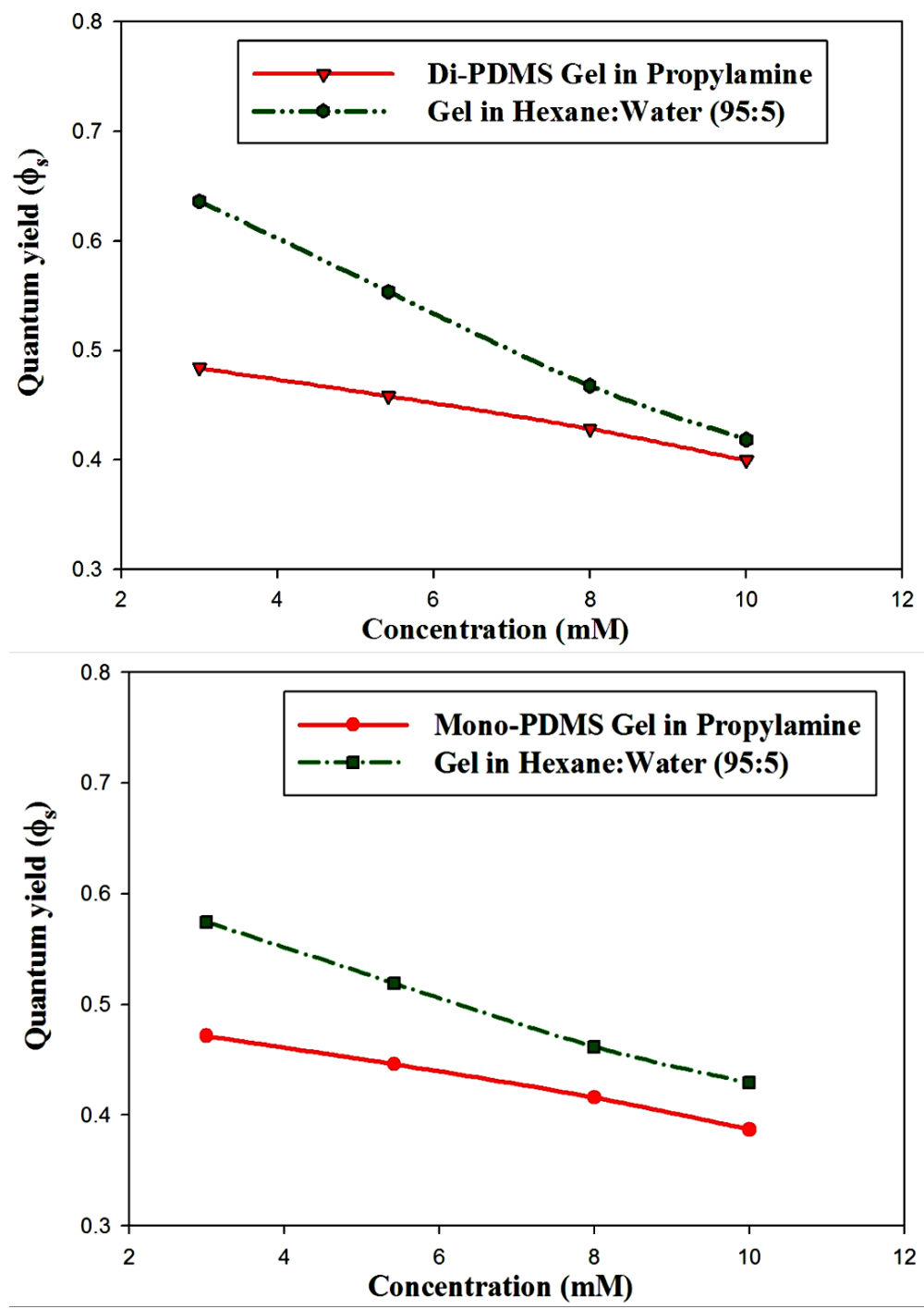

Figure 4.17 Quantum Yields for Mono- and Di-PDMS Gels with propylamine and hexane/water mixtures

\subsubsection{Rheology}

The variations of G' and G" with frequency are shown in Figure 4.18 for the Mono- and Di-PDMS based gels with propylamine and hexane/water: 95/5 mixture. The modulus G' being higher than G" and the constancy confirms the formation of the gels. It is also seen that the moduli are smaller for both Mono- and Di-PDMS based gels, with hexane/water mixture. 

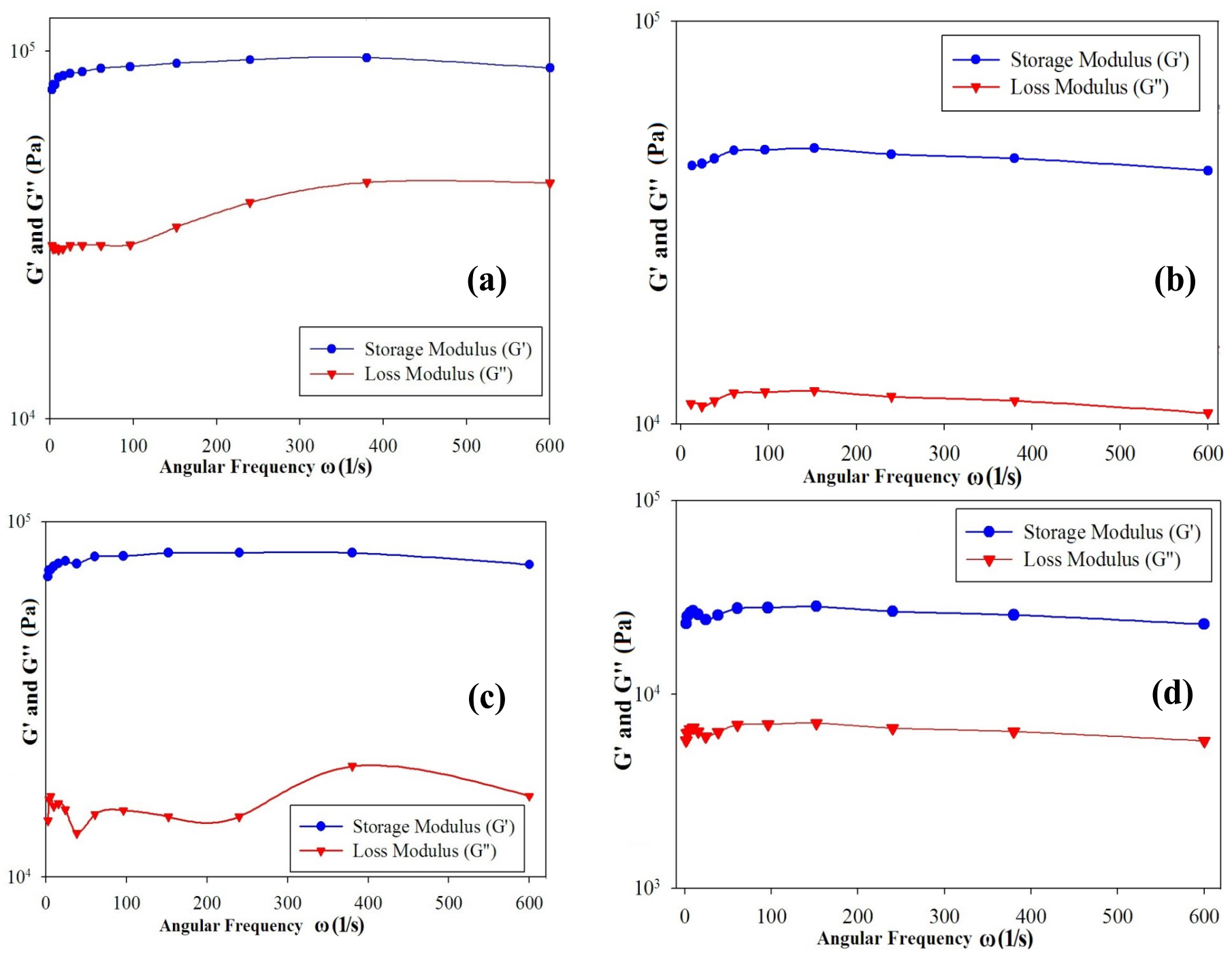

Figure 4.18 Storage and loss moduli of the gels (a) Di-PDMS/propylamine (b) Di-PDMS/

(hexane/water: 95/5) (c) Mono-PDMS/propylamine (d) Mono-PDMS/ (hexane/water: 95/5) 


\subsection{Conclusions}

The present work shows that perylene imides substituted with PDMS either on one imide nitrogen (Mono-PDMS) or on both (Di-PDMS) form organo-gels although there is no hydrogen bonding group in the structure. This is in contrast to the studies reported so far on PTCDI based gels, all of which included a hydrogen bonding moiety. We attribute this to the entanglement of the PDMS, in addition to the $\pi$-interaction mediated self-assembly of the perylene segments. We also presented the first case of the gelation involving mono-substituted PDI (Mono-PDMS), in which the hollow sphere morphology is seen. The morphology of Di-PDMS based gels consist of ribbons, which tend not to be flat, but are folded along the length into an eaves trough. Following the rationalization that we offered in our previous work for the case of a hydrogen-bond mediated organo-gel, the folding is attributed to the asymmetry of interactions between the molecules in the three packing directions: predominant $\pi \ldots \pi$ and van der Waals interactions along one direction and just the latter along the two other directions. The TEM images showed that the fine structure of the ribbons consist of strands of about $1 \mathrm{~nm}$ thick.

Apart from gelation without the presence of hydrogen bonds, gels with Mono-PDMS and Di-PDMS could be made with solvents in which both these solutes dissolve at room temperature. Adding water as a non-solvent at room temperature leads to precipitation. However, it appears that upon heating the solution of these molecules with hexane or diisopropylamine, the PTCDI segment phase separates, and adding water triggers the phase separation of PDMS segment also, and leads to gelation. This type of behavior provides more options in terms of selection of solvents for preparing gels. 


\subsection{References}

1. Law, K. Y. Organic Photoconductive Materials: Recent Trends and Developments.

Chem. Rev. 1993, 93, 449-486.

2. Schmidt-Mende, L.; Fechtenkötter, A.; Müllen, K.; Moons, E.; Friend, R. H.; MacKenzie, J. D. Self-Organized Discotic Liquid Crystals for High-Efficiency Organic Photovoltaics . Science 2001, 293, 1119-1122.

3. Klebe, G.; Graser, F.; Hädicke E. and Berndt, J. Crystallochromy as a Solid-State Effect: Correlation of Molecular Conformation, Crystal Packing and Color in Perylene-3, 4:9, 10-bis (dicarboximide) Pigments. Acta Crystallogr., Sect. B. 1989, 45,69-77.

4. Struijk, C. W.; Sieval, A. B.; Dakhorst, J. E. J.; Van Dijk, M.; Kimkes, P.; Koehorst, R. B. M.; Donker, H.; Schaafsma, T. J.; Picken, S. J.; vande Craats, A. M.; Warman, J. M.; Zuilhof, H.; Sudhölter, E. J. R. Liquid Crystalline Perylene Diimides: Architecture and Charge Carrier Mobilities. J. Am. Chem. Soc. 2000, 122, 11057-11066.

5. Balakrishnan, K.; Datar, A.; Oitker, R.; Chen, H.; Zuo, J.; Zang, L. Nanobelt SelfAssembly From an Organic n-type Semiconductor: Propoxyethyl-PTCDI. J. Am. Chem. Soc. 2005, 127, 10496-10497.

6. Balakrishnan, K.; Datar, A.; Naddo, T.; Huang, J.; Oitker, R.; Yen, M.; Zhao, J.; Zang, L. Effect of Side-Chain Substituents on Self-Assembly of Perylene Diimide Molecules: Morphology Control. J. Am. Chem. Soc., 2006, 128, 7390-7398.

7. Yagai, S.; Monma, Y.; Kawauchi, N.; Karatsu, T.; Kitamura, A. Supramolecular Nanoribbons and Nanoropes Generated from Hydrogen-bonded Supramolecular Polymers Containing Perylene Bisimide Chromophores. Org. Lett. 2007, 9, 1137-1140. 
8. Seki, T.; Maruya, Y.; Nakayama, K.; Karatsu, T.; Kitamura, A.; Yagai, S. Solution Processable Hydrogen-bonded Perylene Bisimide Assemblies Organizing into Lamellar architectures. Chem. Commun., 2011, 47, 12447-12449.

9. Yao, D.; Bender, T. P.; Gerroir, P. J.; Sundararajan, P. R. Self-Assembled Vesicular Nanostructures of Perylene End-Capped Poly(dimethylsiloxane). Macromolecules 2005, 38, $6972-6978$.

10. Yao, D.; Tuteja, B.; Sundararajan, P. R. Pigment-mediated Nanoweb Morphology of Poly(dimethylsiloxane)-sunstituted Perylene Bisimides. Macromolecules 2006, 39, 7786-7788.

11. Sreedevi T.; Ramya Devi. D.; Vedha Hari. B. N. An Emerging Era in Topical Delivery: Organogels. International Journal of Drug Development \& Research 2012, 4, 35-40.

12. Garg, T; Bilandi, A; Kapoor, B; Kumar, S; Joshi, R Organogels: Advanced and Novel Drug Delivery System. International Research Journal of Pharmacy 2011, 2, 15-21.

13. Díaz, D. D.; Kühbeck, D.; Koopmans, R. J. Stimuli-Responsive Gels as Reaction Vessels and Reusable Catalysts. Chem. Soc. Rev., 2011, 40, 427-448.

14. Krishnan, A.S.; Roskov, K. E.; Spontak, R. J. Nanostructured Organogels via Molecular Self-Assembly. in Advanced Nanomaterials Geckeler, K. E.; Nishide, H. Eds. 2010, $2,791-834$.

15. Vintiloiu, A.; Leroux, J-C. Organogels and Their Use in Drug Delivery - A review. Journal of Controlled Release 2008, 125, 179-192.

16. Fujita, N.; Mukhopadhyay, P.; Shinkai, S. Recent Development of Organogels Towards Smart and Soft Materials. Annual Review of Nano Research 2006, 1, 385-428. 
17. George, M.; Weiss, R.G. Molecular Organogels. Soft Matter Comprised of LowMolecular-Mass Organic Gelators and Organic Liquids. Accounts of Chemical Research 2006, 39, 489-497.

18. Abdallah, D. J.; Weiss, R. G. Organogels and Low Molecular Mass Organic Gelators. Advanced Materials 2000, 12, 1237-1247.

19. Terech, P.; Weiss, R.G. Low Molecular Mass Gelators of Organic Liquids and the Properties of Their Gels. Chem. Rev. 1997, 97, 3133-3159.

20. Ajayaghosh, A.; George, S. J. First Phenylenevinylene Based Organogels: SelfAssembled Nanostructures via Cooperative Hydrogen Bonding and $\pi$-Stacking. $J$. Am. Chem. Soc. 2001, 123, 5148-5149.

21. Ajayaghosh, A.; Praveen, V. K. $\pi$-Organogels of Self-Assembled p-Phenylenevinylenes: Soft Materials with Distinct Size, Shape, and Functions. Acc. Chem. Res.2007, 40, 644656.

22. Sugiyasu, K.; Fujita, N.; Shinkai, S. Visible-Light-Harvesting Organogel Composed of Cholesterol-Based Perylene Derivatives. Angew. Chem. Int. Ed. 2004, 43, 1229-1233.

23. Würthner, F.; Hanke, B.; Lysetska, M.; Lambright, G.; Harms, G. S. Gelation of a Highly Fluorescent Urea-Functionalized Perylene Bisimide Dye. Org. Lett. 2005, 7, 967-970.

24. Li, X.Q.; Stepanenko, V.; , Chen, Z.; Prins, P.; Siebbeles, L.D.; Würthner, F. Functional Organogels From Highly Efficient Organogelator Based on Perylene Bisimide Semiconductor. Chem. Commun (Camb). 2006, 37, 3871-3873.

25. Wu, H. X.; Xue, L.; Shi, Y.; Chen, Y. L; Li, X. Y. Organogels Based on J- and H-Type Aggregates of Amphiphilic Perylenetetracarboxylic Diimides. Langmuir 2011, 27, 30743082. 
26. Díaz, D. D.; Cid, J. J.; Vázquez, P.; Torres, T. Strength Enhancement of Nanostructured Organogels Through Inclusion of Phthalocyanine-Containing Complementary Organogelator Structures and In Situ Cross-Linking by Click Chemistry. Chem. Eur. J. 2008, 14, 9261 - 9273.

27. Díaz, D. D.; Torres, T.; Zentel, R.; Davis, R.; Brehmer, M. Physicochemical Characterization of Octakis(alkyloxy)-substituted Zn(II)-phthalocyanines Non-covalently Incorporated into an Organogel and Their Remarkable Morphological Effect on the Nanoscale-Fibers. Chem. Commun., 2007, 2369-2371.

28. Dahan, E.; Sundararajan, P. R. Thermo-reversible Physical Gels of Poly(dimethylsiloxane) without Cross-Links or Functionalization. Langmuir 2013, 29, $8452-8458$.

29. Lee, J. N.; Park, C.; Whitesides, G. M. Solvent Compatibility of Poly (dimethylsiloxane)Based Microfluidic Devices. Anal. Chem. 2003, 75, 6544-6554.

30. Khanna, S.; Khan, M. K.; Sundararajan, P. Influence of Double Hydrogen Bonds and Alkyl Chains on the Gelation of Non-chiral Polyurethane Model Compounds: Sheets, Eaves Trough, Tubes and Oriented Fibers. Langmuir, 2009, 25, 13183-13193.

31. Schnur, J. M.; Ratna, B. R.; Selinger, J. V.; Singh, A.; Jyothi, G.; Easwaran, K. R. K. Diacetylenic Lipid Tubules: Experimental Evidence for a Chiral Molecular Architecture. Science 1994, 264, 945-947.

32. Schnur, J.M. Lipid tubules: A Paradigm for Molecularly Engineered Structures. Science 1993, 262, 1669-1676.

33. Helfrich, W.; Prost, J. Intrinsic Bending Force in Anisotropic Membranes Made of Chiral Molecules. Phys. Rev. A. 1988, 38, 3065 -3068. 
34. Fetters, L. J.; Lohse, D. J.; Scott T. Milner, S. T.; William W. Graessley, W. W. Packing Length Influence in Linear Polymer Melts on the Entanglement, Critical, and Reptation Molecular Weights. Macromolecules 1999, 32, 6847-6851.

35. Fetters, L. J.; Lohse, D. J.; Richter, D.; Witten, T. A.; Zirkel, A. Connection between Polymer Molecular Weight, Density, Chain Dimensions, and Melt Viscoelastic Properties Macromolecules, 1994, 27, 4639-4647. 


\section{CHAPTER 5}

The Thermo-reversible Gelation of PTCDI Mono/Di- Substituted with Ethylene Oxide/ Propylene Oxide (Jeffamine ${ }^{\circledR}$ ) Co-oligomer

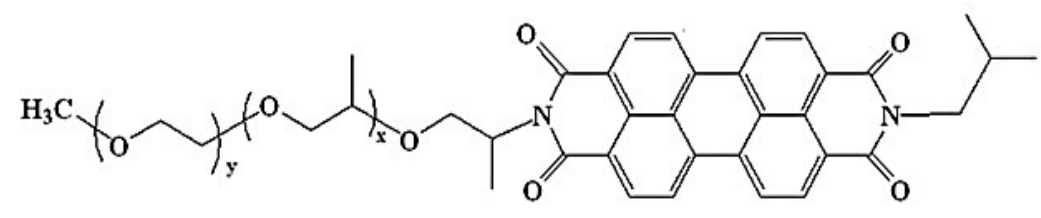
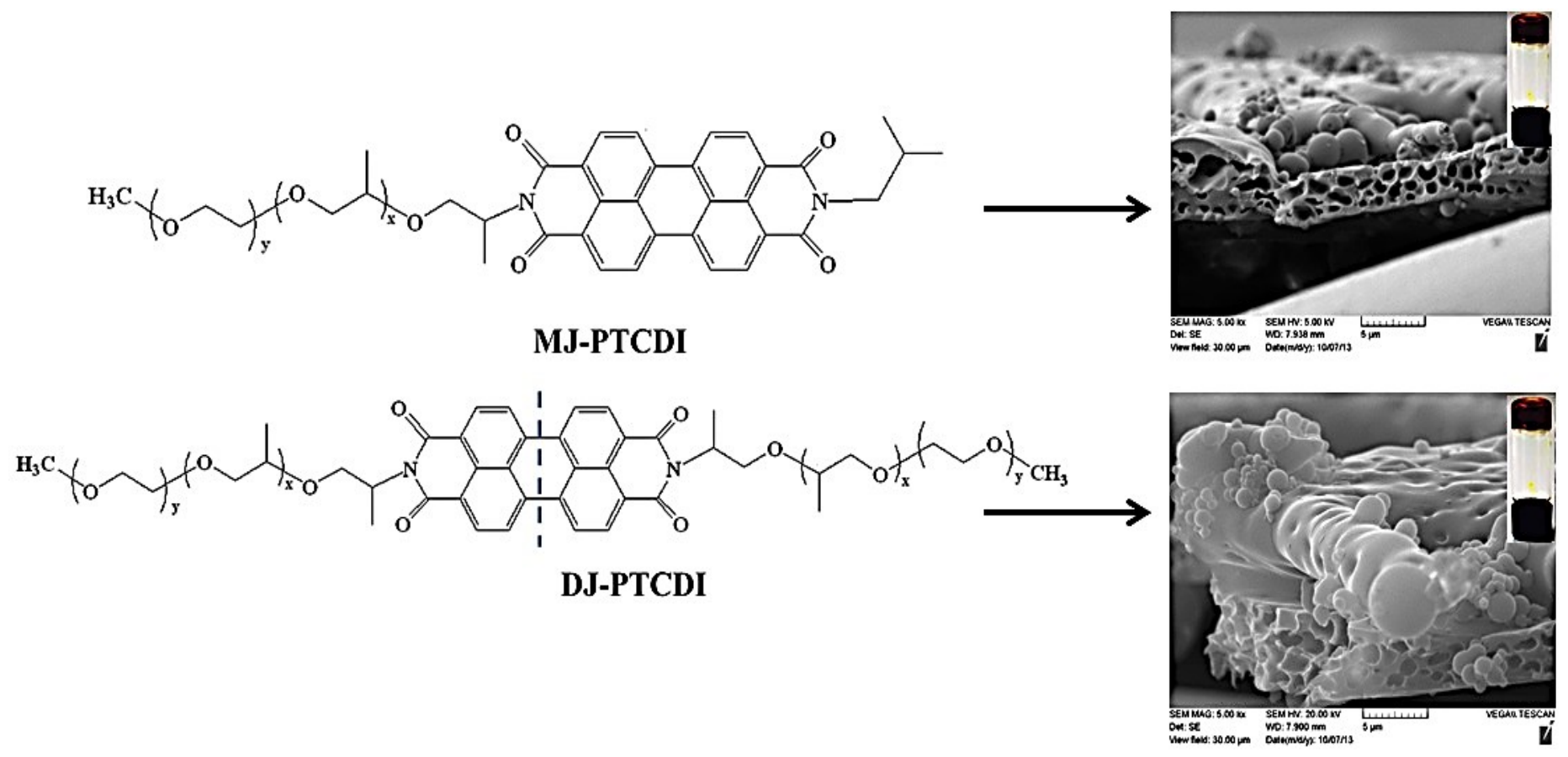


\subsection{Introduction}

Self-assembly of perylene diimide derivatives in solution leads to nanostructures resulting in nanobelts, ${ }^{1,2}$ nanotubes, ${ }^{3,4}$ and nanofibers. ${ }^{5}$ While the $\pi$-interactions of the perylene units enable the self-assembly of these chromophores, the nature of the assembly can be modulated by substitution of side groups. ${ }^{6-9}$ Short linear side groups give rise to liquid crystalline properties ${ }^{10,11}$ and crystallization results in morphologies consisting of nanowires. On the other hand, it has been shown that attachment of longer chains to perylene bisimide impedes liquid crystalline ordering. ${ }^{12}$ As mentioned in Chapter 4, the role of perylene diimides as organo gelators has been examined by a few authors, including us. ${ }^{2}$ In the design of the molecules, other authors introduced hydrogen bonding groups to the structure to augment the $\pi$-interaction for self-assembly leading to gelation. In the last chapter we showed that the PDMS-PTCDI and PDMS-PTCDI-PDMS molecules can lead to gels, without hydrogen bonding groups in the structure. We proposed that the interaction between the PDMS chains would be sufficient to cause gelation. The length of the side chains (PDMS) varied from 10 to 40 siloxane units, in addition to the methylene groups. In this chapter, we used a water-soluble side chain with PTCDI. Here we describe the gelation caused by PTCDI, substituted with Jeffamine ${ }^{\circledR}$ on one imide nitrogen (MJ-PTCDI) or both (DJ-PTCDI), as shown in Scheme 5.1. We used Jeffamine M-2070 polyetheramine, (also shown in Scheme 5.1) which is a propylene oxide/ethylene oxide copolymer (PO/EO mole ratio of 10/31) with $\mathrm{M}_{\mathrm{w}}=2000$ (Huntsman Corporation Technical Bulletin). In this case again, there is no hydrogen bonding group $(\mathrm{N}-\mathrm{H}, \mathrm{C}=\mathrm{O})$ in the side chain structure. 


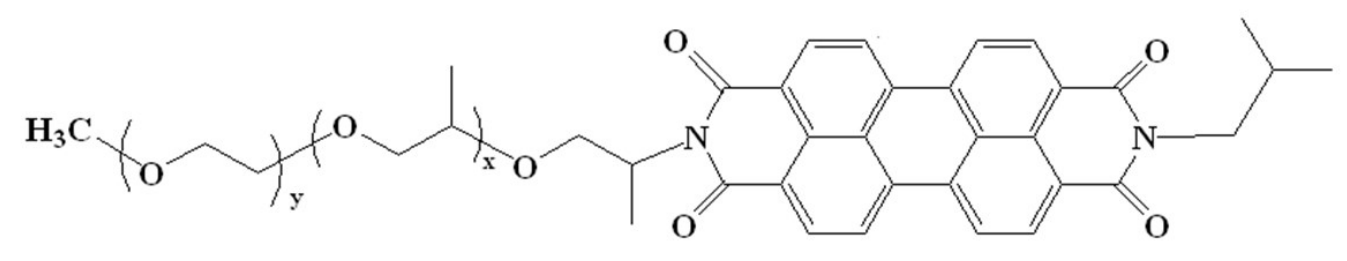

(a) MJ-PTCDI

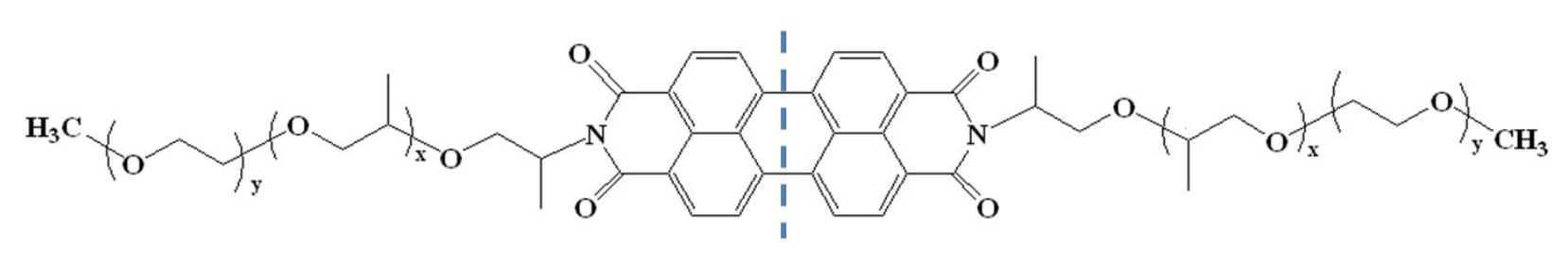

(b) DJ-PTCDI

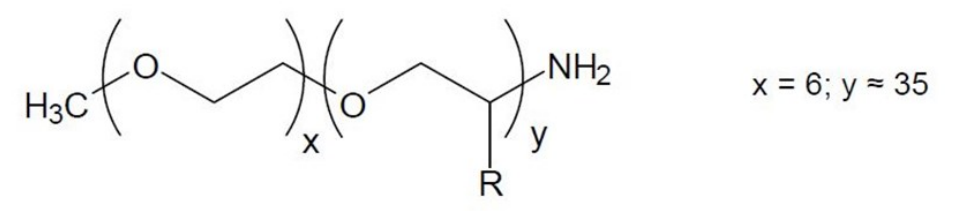

$\mathrm{R}=\mathrm{H}$ for $(\mathrm{EO})$, or $\mathrm{CH}_{3}$ for $(\mathrm{PO})$

\section{Jeffamine $2070^{\circledR}$}

Scheme 5.1 PTCDI substituted with Jeffamine ${ }^{\circledR}$ on one imide nitrogen (a) (MJ-PTCDI) or both (b) (DJ-PTCDI). The dashed line demonstrates the symmetry of DJ-PTCDI

As with the Di-PDMS and Mono-PDMS described in the last chapter, the DJ-PTCDI is a Gemini surfactant and MJ-PTCDI is an inverse macromolecular surfactant.

\subsection{Experimental}

The synthesis of MJ-PTCDI and DJ-PTCDI has been described by Islam et al. ${ }^{12}$ The synthetic route is given in Appendix 2. MJ-PTCDI and DJ-PTCDI gels of different concentrations (3.00-10.0 mM) were prepared by dissolving the solutes in the required amount of 
propylamine at temperatures ranging from $56-65{ }^{\circ} \mathrm{C}$ with constant stirring; this resulted in a red

solution. Closed vials were used to avoid the evaporation of the solvent. The gels were prepared by adding different water concentrations ranging from $1-5 \%$ and slow cooling the solution to room temperature. Gelation was confirmed by tube inversion, i.e., no flowing solvent. Xerogels were prepared by drying the gels at room temperature for a few days and then under vacuum overnight for complete removal of solvents. The same procedure was followed to prepare gels from cyclohexane/toluene and cyclohexane/ toluene mixtures, at different non-solvent: solvent ratios ranging from 1 to $5 \%$ solvent.

\subsection{Results and Discussion}

Polyethylene glycol belongs to a family of polymers that form hydrogels which have numerous advantages with respect to their role as delivery vehicles; with numerous hydrogel systems it has been shown that one can control the release characteristics through systematic changes in the gel's physical and chemical structure ${ }^{13}$. With respect to tailoring gel properties, those derived from synthetic polymers, such as poly (ethylene glycol) (PEG), poly(vinyl alcohol) (PVA), poly(N-isopropylacrylamide) (PNIPAAM), are particularly useful and have been used in numerous regenerative medicine applications ${ }^{14}$. Of these, the non-ionic, hydrophilic PEG gel systems are increasingly used; over the past few decades, poly(ethylene glycol) (PEG) hydrogels have been extensively used as matrices for controlling drug delivery, as well as cell delivery

vehicles for promoting tissue regeneration ${ }^{15}$. The versatility of the PEG macromer chemistry ${ }^{16}$, together with its excellent biocompatibility, has spurred the development of numerous intelligently-designed hydrogel systems for regenerative medicine applications ${ }^{17}$. 
In the present case, the $\mathrm{PO} / \mathrm{EO}$ copolymer is attached to perylene diimide, which is not soluble in any common solvents. However, both MJ-PTCDI and DJ-PTCDI are moderately soluble in water, and in acetone, $\mathrm{CH}_{2} \mathrm{Cl}_{2}$, chloroform, DMAc, DMF, methanol, and THF. Previous studies $^{12}$ in our group showed that self-assembly of the PTCDI was seen in water and aqueous solvent mixtures, but not in the other non-aqueous solvents. Aggregation was seen beginning with concentrations of $10^{-7} \mathrm{M}$, and no dissociation was seen up to $90^{\circ} \mathrm{C}$.

\subsubsection{Gelation Characteristics}

In our search for a suitable solvent for gelation, non-aqueous solvents did not lead to a gel. For example, propylamine that we used for PDMS based molecules, dissolved both MJPTCDI and DJ-PTCDI above $65{ }^{\circ} \mathrm{C}$, but precipitation occurred upon cooling. However, upon adding $1 \%(\mathrm{v} / \mathrm{v})$ water to the hot solution resulted in a gel upon cooling. There have been a few publications recently ${ }^{18,19}$ on the phenomenon of non-solvent induced self-assembly and nanofibre formation of molecules such as derivatives of phthalocyanines and porphyrins. In these cases, typically a non-solvent was added at a slow controlled rate to a solution of these molecules. In the present case, water, the solvent, causes gelation upon addition to the nonaqueous solution.

In addition to propylene/ water mixture, we found that gelation occurs with cyclohexane/ toluene, with cyclohexane as the major component. The solution/gelation diagram is shown in Figure 5.1 for MJ-PTCDI and DJ-PTCDI with propylamine and cyclohexane as major solvent components and with 1 and 5\%(v/v) water. In all these cases the minimum solute concentration, also known as the critical gelation concentration (CGC) is $3 \mathrm{mM}$, below which the gelator is soluble, existing primarily as monomers in solution. We find a distinct difference between MJ- 
PTCDI and DJ-PTCDI in the concentration dependence of solution and gelation with temperature. With the former, the solution temperature shows a maximum at a concentration of about $5 \mathrm{mM}$, whereas with DJ-PTCDI, it is about $8 \mathrm{mM}$. This is attributed to the higher molecular weight of DJ-PTCDI. The maximum in the gelation curve, however, is about $8 \mathrm{mM}$ for both. Further, the onset of gelation occurs at a lower temperature with an increase in water content. For example, for MJ-PTCDI, with 99/1 (v/v) propylamine/ water, the onset of gelation with $8 \mathrm{mM}$ concentration is seen at $40{ }^{\circ} \mathrm{C}$, and it reduces to $35^{\circ} \mathrm{C}$ with $95 / 5$ ratio. 

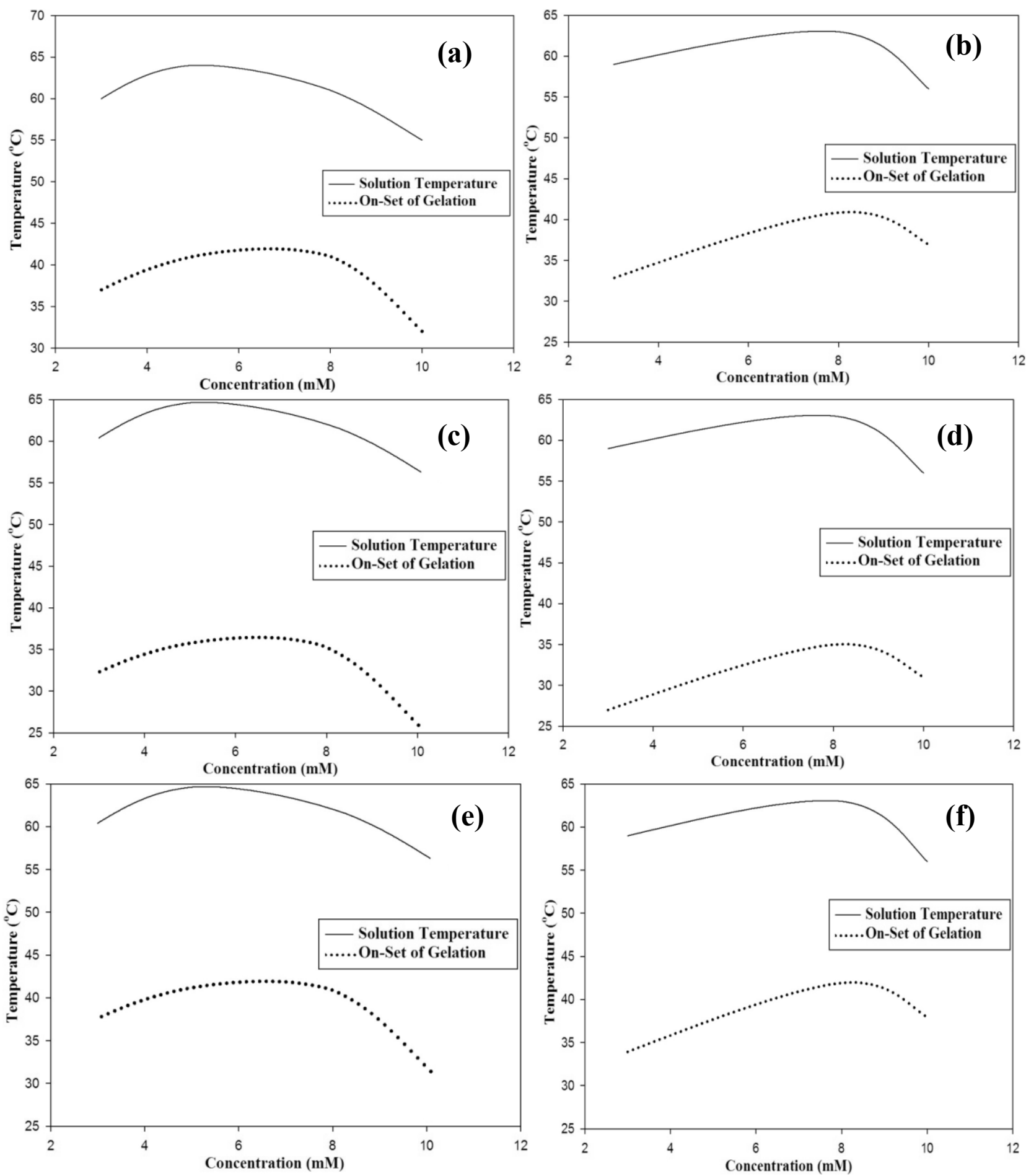

Figure 5.1 (a) MJ-PTCDI with propylamine-water (99/1 v/v) (b) DJ-PTCDI with propylaminewater $(99 / 1 \mathrm{v} / \mathrm{v})$ (c) MJ-PTCDI with propylamine-water $(95 / 5 \mathrm{v} / \mathrm{v})$ (d) DJ-PTCDI with propylamine-water $(95 / 5 \mathrm{v} / \mathrm{v})(\mathrm{e}) \mathrm{MJ}-\mathrm{PTCDI}$ with cyclohexane-toluene $(95 / 5 \mathrm{v} / \mathrm{v})(\mathrm{f}) \mathrm{DJ}-\mathrm{PTCDI}$ with cyclohexane-toluene $(95 / 5 \mathrm{v} / \mathrm{v})$ 


\subsubsection{Morphology}

Figures 5.2 (a), (b), (c) show the OM, SEM and TEM images of a $10.00 \mathrm{mM}$ DJ-PTCDI gel formed with propylamine-water $(99 / 1 \mathrm{v} / \mathrm{v})$. All the images show spherical domains of about the same size aggregating predominantly in one direction to form fiber like morphology. In particular, the OM image in Figure 5.3 (a) shows that the spheres are highly organized into long fibres of several hundred microns in length. We further note that these spheres are hollow, and are large vesicles. This is confirmed by the SEM of the cross section. A piece of gel was dipped in liquid nitrogen, cut, and the fractured morphology was then observed in the SEM. It is seen in Figure $5.3(\mathrm{~g})$ that the vesicles are fused together, forming a honeycomb morphology. This is the vesicular analogue of worm-like micelles. The TEM image in Figure 5.2 (c) also shows the fusion of the vesicles. The smallest of the vesicles is about $50 \mathrm{~nm}$ in diameter. In all the publications on similar disubstituted PTCDI's so far, nano-fiber morphology was seen. Note that in those cases, one substituent was hydrophilic and the other was hydrophobic. With DJ-PTCDI, the substituents on both sides are hydrophilic. This would lead to a vesicular morphology similar to that of phospholipids. Two possible models for the packing is shown in Figure 5.3.

The gelation of mono-substituted PDI has not been reported so far. The OM and SEM images of MJ-PTCDI based gels are shown in Figures 5.2 (d) and (e). This gel also shows vesicular morphology, as seen from the TEM image in Figure 5.2 (f). The diameter varies from 50 to $100 \mathrm{~nm}$. Although fibres as in the OM of DJ-PTCDI are not seen here, the "chaining" of the spheres seems to be present, as indicated by the yellow line in Figure 5.2 (d). The SEM image of the cross section of the sample in Figure 5.2 (h) shows the honeycomb morphology similar to that in Figure 5.2 (g) for DJ-PTCDI. Thus with MJ-PTCDI based gels also, the wormlike vesicular morphology occurs. The association to form long fibers was seen in our previous 
study of the drop-cast films of DJ-PTCDI and MJ-PTCDI and the secondary aggregation of the primary spheres continued to form such long fibers. Formation of worm-like micelles was reported recently, during the association of Gemini surfactants ${ }^{26}$.

The other non-solvent/solvent combinations also led to gelation. Figure 5.4 shows the SEM images of the spherical morphology of DJ-PTCDI and MJ-PTCDI with cyclohexane/ toluene $(95 / 5 \mathrm{v} / \mathrm{v})$ and cyclohexane/toluene $(99 / 1 \mathrm{v} / \mathrm{v})$. Clustering is seen in Figures 5.4 (a) and (b). Thus, with hydrophilic Jeffamine on one side or both sides (Gemini), the surface interactions between the vesicles result in clustering and honeycomb morphology. Another observation was that when less good solvent, such as toluene was used $(99 / 1 \mathrm{v} / \mathrm{v}$ cyclohexane/toluene) the chain clustering was enhanced as seen in Figure 5.4 (e)-(g). This led us to conclude that increasing the amount of good solvent weakens the gel. This is also reflected in the rheology measurements in section 5.3.4. 

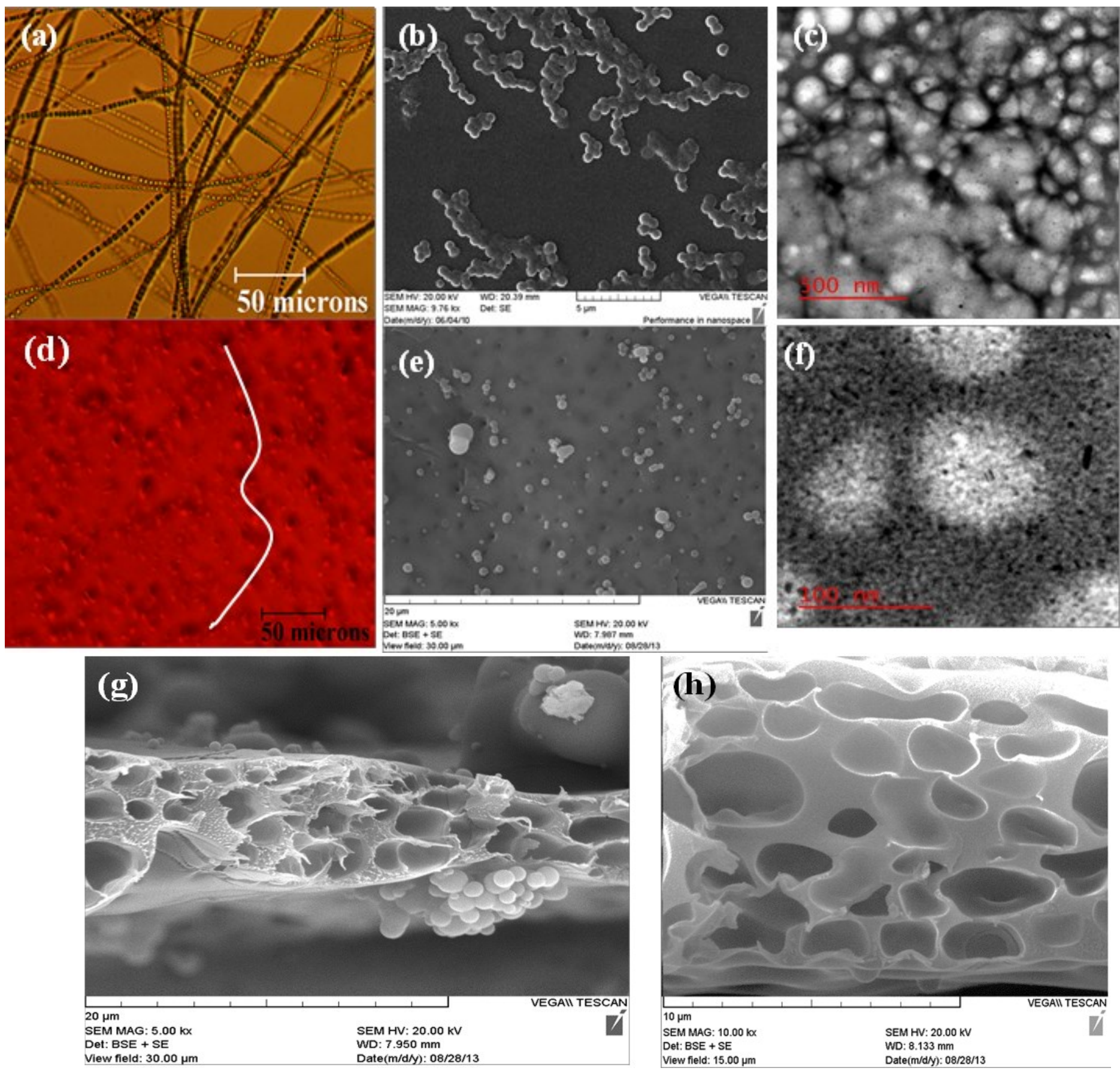

Figure 5.2 (a), (b) and (c): OM, SEM, and TEM, respectively of $10.00 \mathrm{mM}$ DJ-PTCDI gel with

propylamine-water (99/1 v/v); (d), (e) and (f): OM, SEM and TEM, respectively, of $10.00 \mathrm{mM}$

MJ-PTCDI gel with propylamine-water. (g) SEM image of the cross section of DJ-PTCDI gel

(h) cross section of MJ-PTCDI gel 

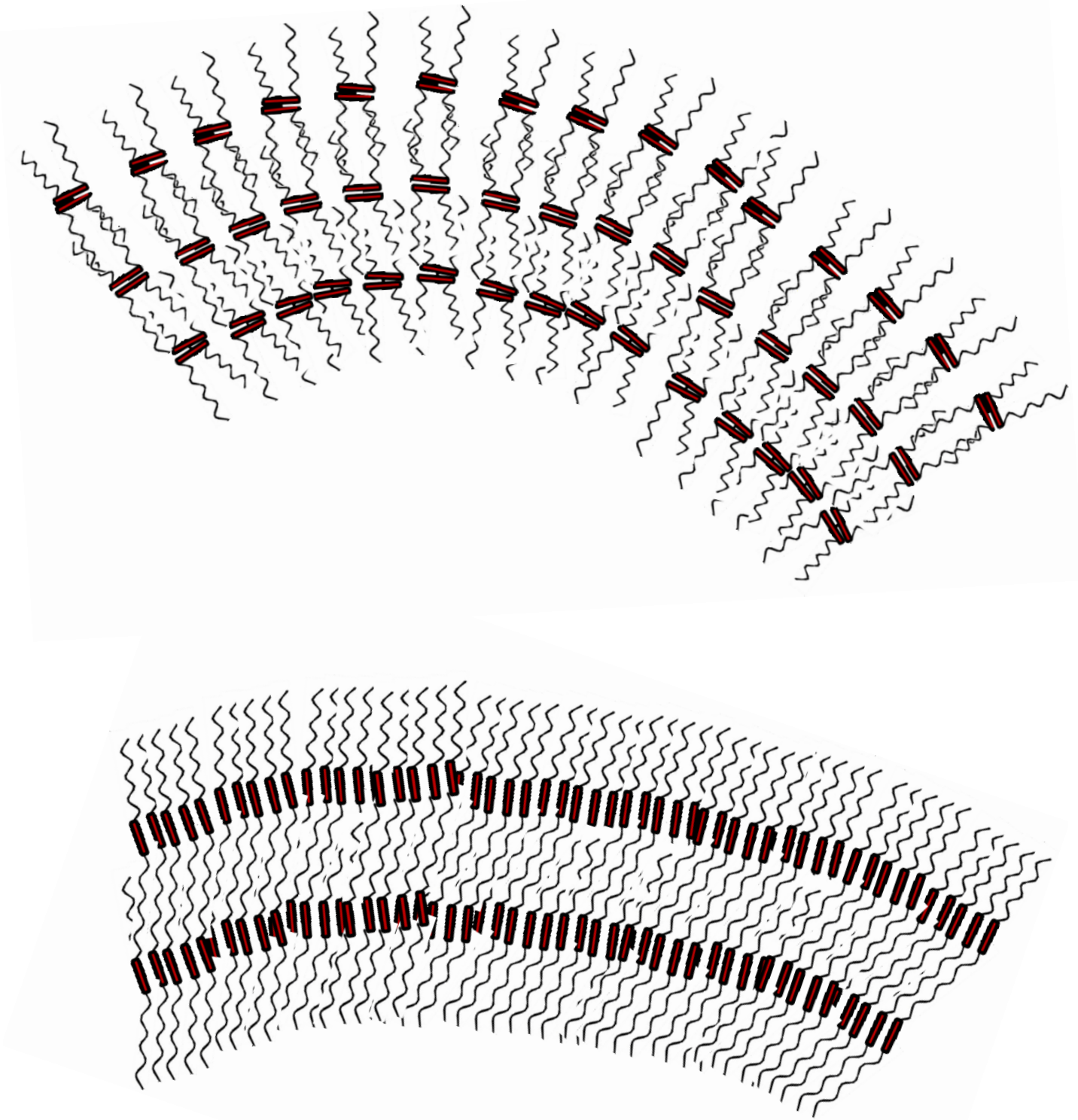

Figure 5.3 Two models of DJ-PTCDI packing; the substituents on bou. . is are hydrophilic. This would lead to a vesicular morphology 

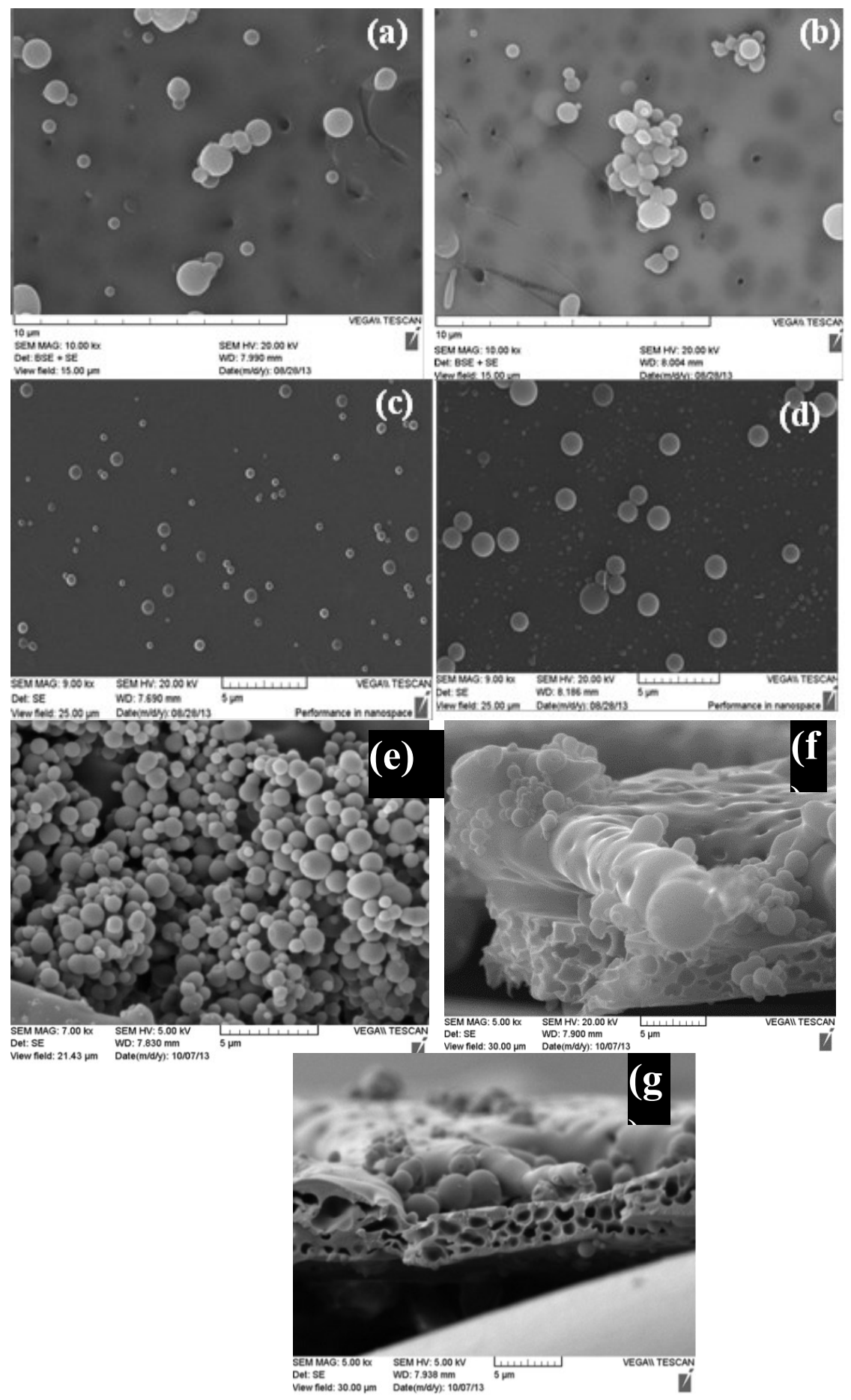

Figure 5.4 SEM of Xerogel formed with cyclohexane/toluene (95/5 v/v) (a) MJ-PTCDI and (b) DJ-PTCDI; (c) MJ-PTCDI (d) DJ-PTCDI; Xerogel with cyclohexane/toluene (99/1 v/v) (e) DJ-PTCDI (f) cross section of DJ-PTCDI and (g) MJ-PTCDI 


\subsubsection{Thermo-reversibility}

The thermo-reversibility of the gels was verified with DSC. The DSC analysis (Figure 5.5 (a)) of the MJ-PTCDI gels showed an endotherm at $42^{\circ} \mathrm{C}$ in the first heating cycle corresponding to the gel-sol transition, and a sol-gel transition exotherm at $30^{\circ} \mathrm{C}$ upon cooling. This shows that the gelation is thermo-reversible. With the scan rate of $5^{\circ} \mathrm{C} / \mathrm{min}$ used here, the sample does not have sufficient time to gel during the cooling cycle and a shallow endotherm for gel-sol transition resulted during the second heating cycle. We found that during the cooling

cycle, it is necessary to maintain the temperature in the DSC close to the sol-gel transition for about 30-45 minutes to observe the well-defined endotherm in the second heating cycle. 

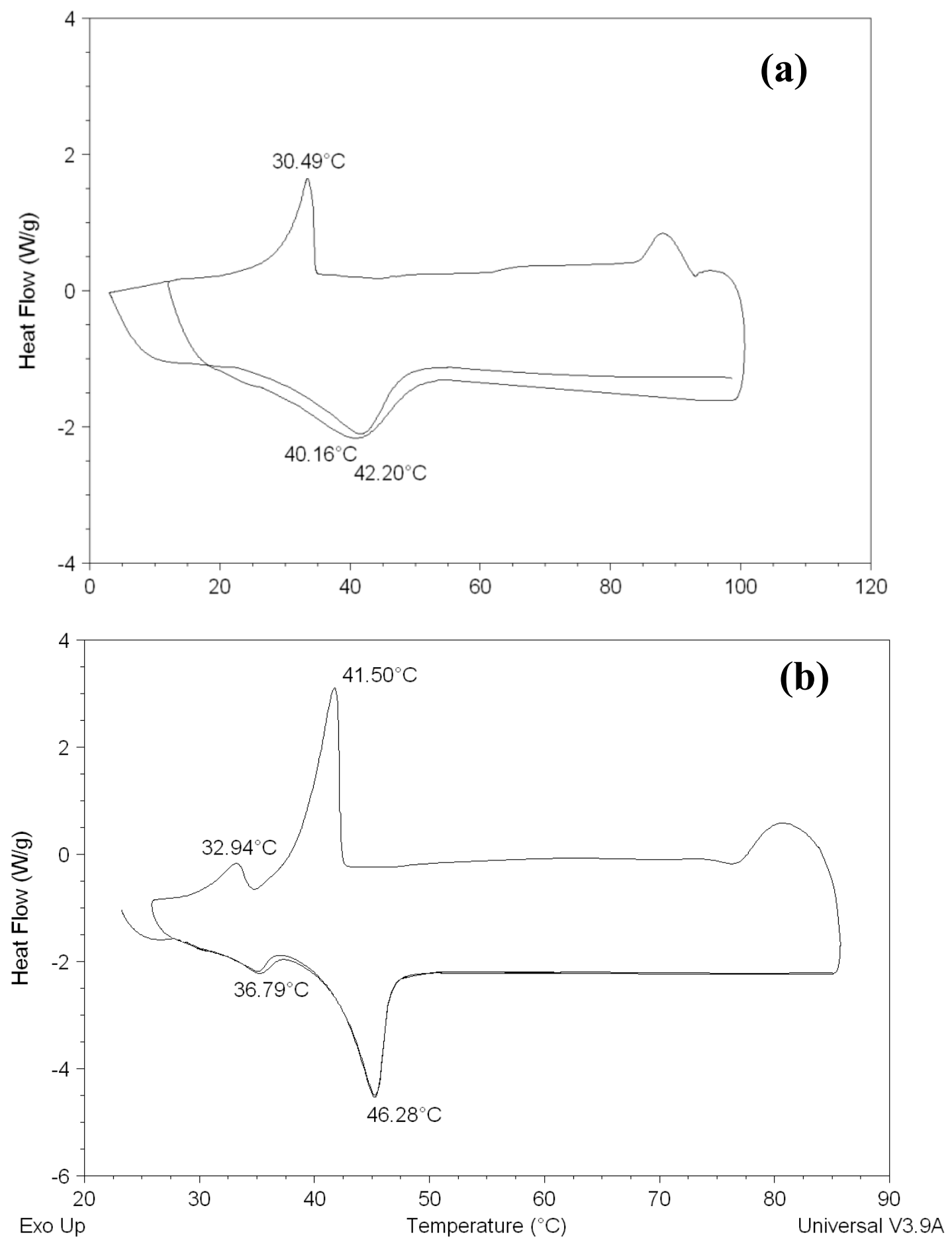

Figure 5.5 DSC of $10.00 \mathrm{mM}$ gels with propylamine-water (99/1 v/v) (a) MJ-PTCDI (b) DJPTCDI 


\subsubsection{Optical Properties}

Generally, an electronic transition is coupled to a single vibration; the intensities of the vibrational transitions follow the order $\mathrm{S}_{0-0}>\mathrm{S}_{0-1}>\mathrm{S}_{0-2}$ for non-aggregated monomers in solution. $^{23,} 24$ Intensity reversal of the peaks at $\mathrm{S}_{0-1}$ or $\mathrm{S}_{0-2}$, is associated with aggregate formation. ${ }^{24}$ It is expected that below the critical gel concentration, the mono- and di-substituted perylene imide show typical monomeric absorption pattern at lower concentrations and an intensity reversal at higher concentration confirming the formation of the gels. Normally at lower concentration if there is no aggregation typical monomeric absorption pattern is expected where most intense peak will be the longer wavelength peak with a very fine vibronic structure in the absorption peak. ${ }^{25}$ This is seen for both the MJ-PTCDI and DJ-PTCDI gels with propylaminewater $(99 / 1 \mathrm{v} / \mathrm{v}$ ) at $1.00 \mathrm{mM}$, where the peaks at 533 (Figure $5.6(\mathrm{a}))$ and $526 \mathrm{~nm}$ (Figure 5.6 (c)), respectively are higher than the peaks at $498 \mathrm{~nm}$ and $491 \mathrm{~nm}$, indicating no aggregation. There is an intensity reversal of these peaks with the $3.00 \mathrm{mM}$ gels, since that was the minimum gel concentration. As concentration increases for MJ-PTCDI gels, the peak at $498 \mathrm{~nm}$ slightly blue shifts to $496 \mathrm{~nm}$ and that at $533 \mathrm{~nm}$ also blue shifts to $531 \mathrm{~nm}$. Also, the intensity of the peak at $496 \mathrm{~nm}$ relative to $531 \mathrm{~nm}$ is much higher (ratio of 1:0.675 for a $10.00 \mathrm{mM}$ gel). This trend is also seen for the DJ-PTCDI gel, the peak at $480 \mathrm{~nm}$ also is a much higher intensity than the peak at $525 \mathrm{~nm}$ (ratio of 1:0.45 for a $10.00 \mathrm{mM}$ gel). As shown in Figures 5.6 (b),(d) the fluorescence spectra of both MJ-PTCDI gels and DJ-PTCDI gels are mirror images of the absorption spectra, with fluorescence quenching going from lower concentration $3.00 \mathrm{mM}$ to higher concentration $10.00 \mathrm{mM}$. The decrease of fluorescent intensity with concentration (Figure 5.6), combined with the blue shift of the maxima from the solution to the gel state 
(Figure 5.7) indicates H-stacking of the perylene units in the gel. This was also observed when cyclohexane-water was used to gelate MJ-PTCDI and DJ-PTCDI (Figure 5.8).
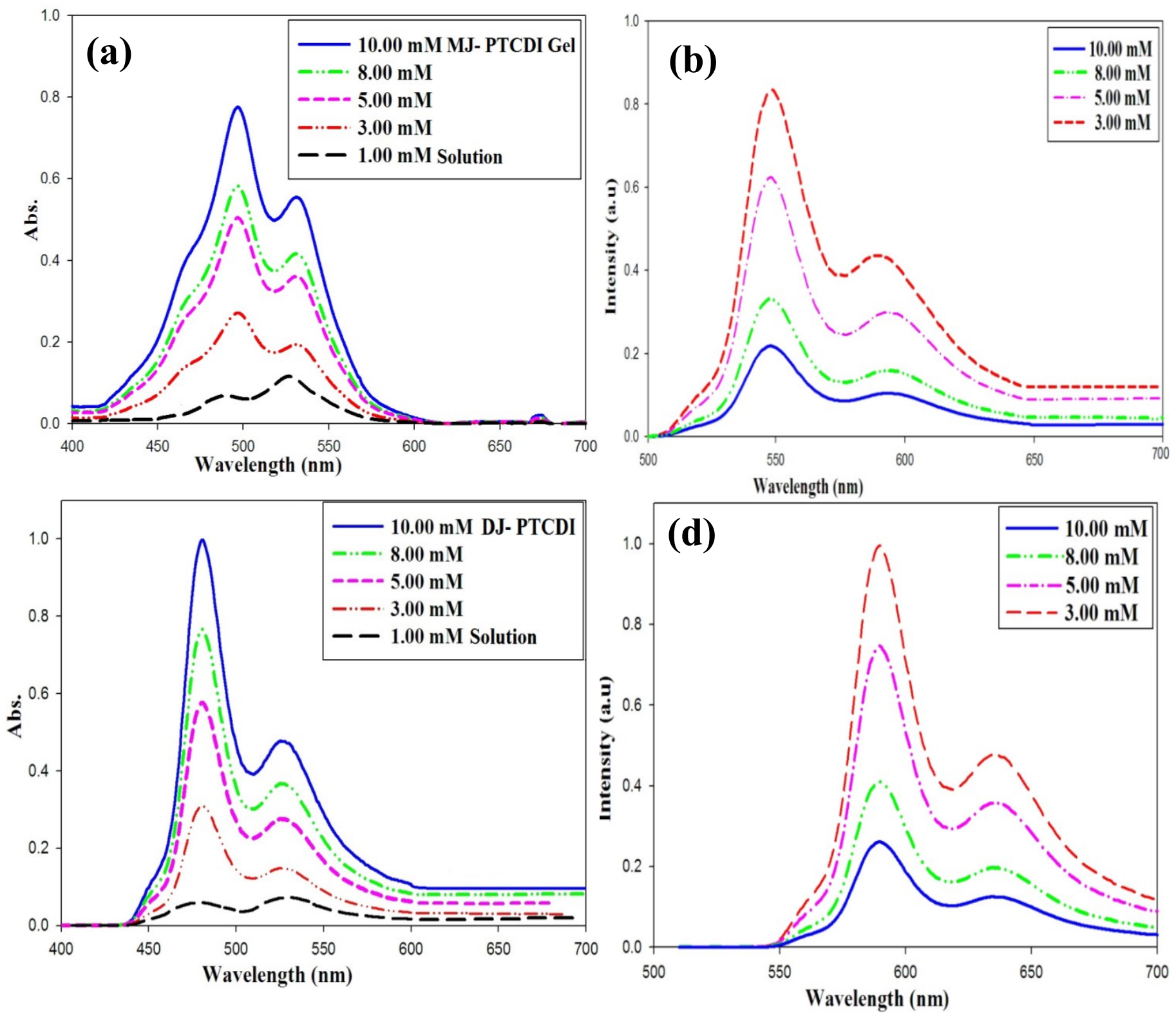

Figure 5.6 (a) UV-Vis absorption (b)Fluorescence of MJ-PTCDI gel with propylamine-water

(99/1 v/v); (c) UV-Vis absorption (d) Fluorescence of DJ-PTCDI gel with propylamine-water $(99 / 1 \mathrm{v} / \mathrm{v})$ 
For both MJ-PTCDI and DJ-PTCDI gels, when the amount of water increases from $1 \%$ to $5 \%$ the peak at the longer wavelength $\left(\mathrm{S}_{0-0}\right)$, increases in intensity and almost has the same intensity as the peak at $\mathrm{S}_{0-1}$ (Figure 5.7). This can be due to the solubility of the Jeffamine ${ }^{\circledR}$ segment with increasing water content.

\subsubsection{Rheology}

The variations of G' and G" with frequency are shown in Figure 5.9 (a)-(g) for the gel samples of MJ-PTCDI and DJ-PTCDI. It is seen that for all the gel samples, the G' is higher than the G" and remains invariant through the frequency range which confirms that the material is a gel. The DJ-PTCDI and MJ-PTCDI gels that were formed with cyclohexane/toluene (99/1 v/v) (Figures 5.9 (e) and (f), respectively) showed higher storage moduli than the gels formed with more toluene $(99 / 5 \mathrm{v} / \mathrm{v})$ (Figures 5.9 (c); (d)); this confirms the initial observation that adding more of a good solvent, weakens the chaining or bundling of the spheres and thus makes for a weaker gel and in this case also the $G^{\prime}$ is higher. 

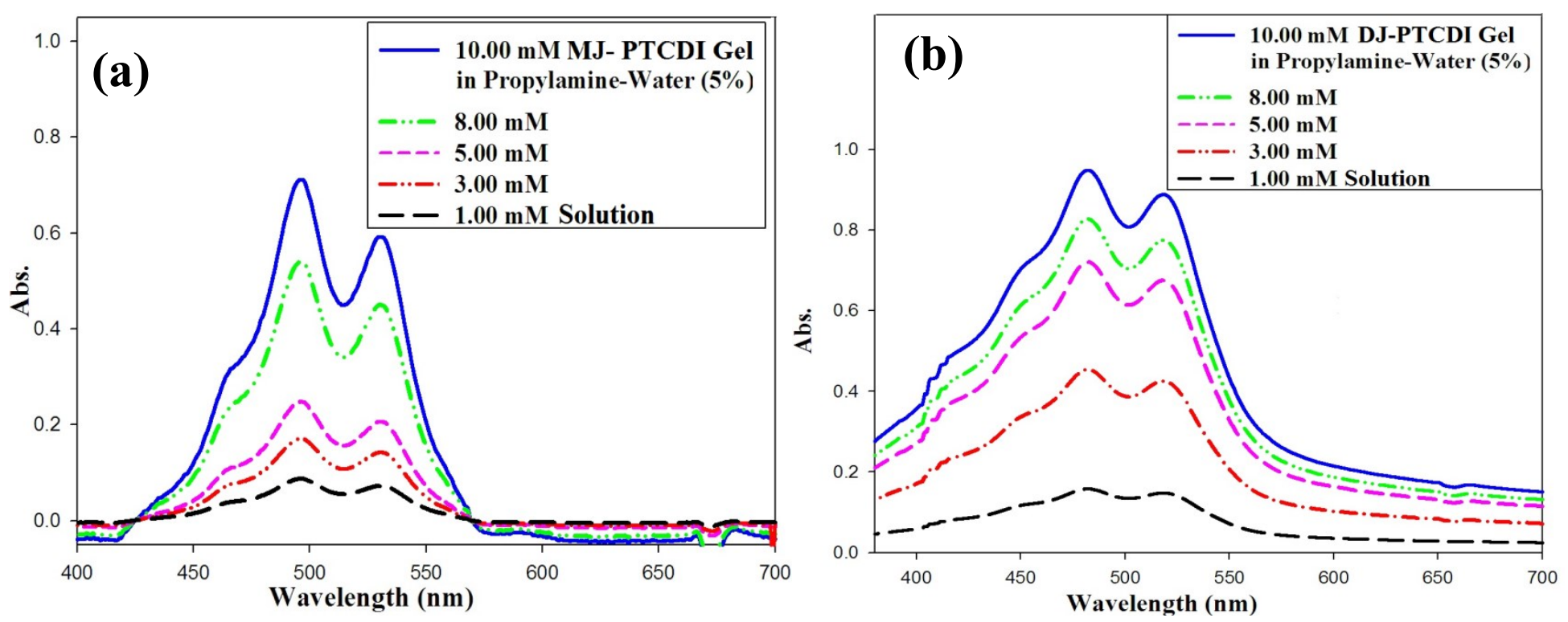

Figure 5.7 UV-Vis absorption (a) MJ-PTCDI gel (b) DJ-PTCDI gel with propylamine-water $(95 / 5 \mathrm{v} / \mathrm{v})$ 

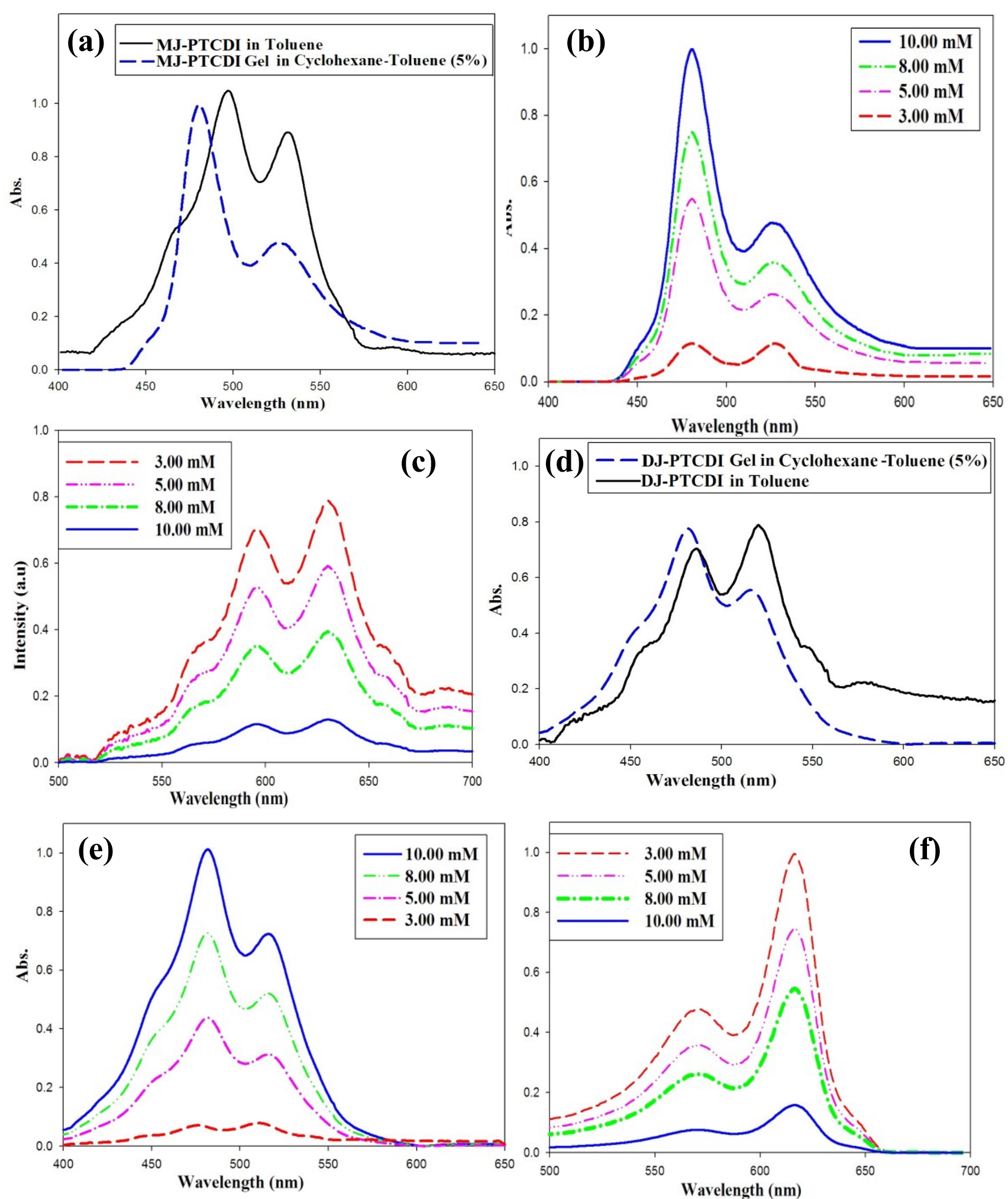

Figure 5.8 (a) $10.00 \mathrm{mM}$ (b) UV-Vis absorption (c) Fluorescence spectra of MJ-PTCDI Gels;

(d)10 mM (e) UV-Vis absorption (f) Fluorescence spectra of DJ-PTCDI Gels ; with cyclohexane/toluene $(95 / 5 \mathrm{v} / \mathrm{v})$ as the solvents for gel formation 

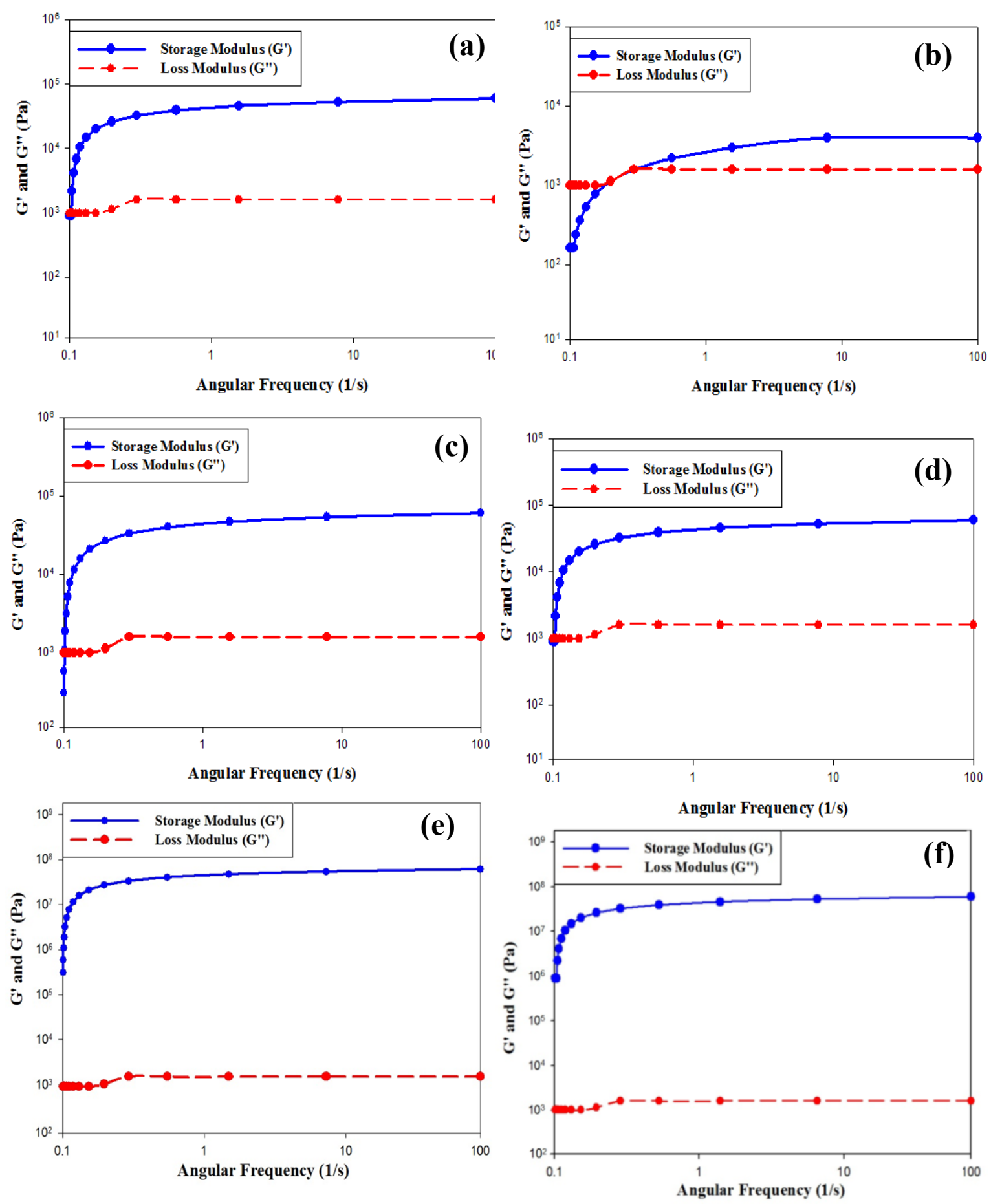

Figure 5.9 Variation of G' and G" with frequency for (a) DJ-PTCDI and (b) MJ-PTCDI Gels with propylamine/water (99/1 v/v); (c) DJ-PTCDI and (d) MJ-PTCDI Gels with cyclohexane/toluene (95/5 v/v); (e) DJ-PTCDI and (f) MJ-PTCDI Gels with cyclohexane/toluene $(99 / 1 \mathrm{v} / \mathrm{v})$ 


\subsection{Conclusions}

In this chapter, we describe the gelation caused by PTCDI, substituted with Jeffamine ${ }^{\circledR}$ on one imide nitrogen (MJ-PTCDI) or both (DJ-PTCDI). Since Jeffamine ${ }^{\circledR}$ is a propylene oxide/ethylene oxide copolymer which is water soluble; thus DJ-PTCDI is a Gemini surfactant and MJ-PTCDI is an inverse macromolecular surfactant. In our previous studies, our group showed that self-assembly of the PTCDI was seen in water and aqueous solvent mixtures, but not in the other non-aqueous solvents. In this chapter we show that a good solvent such as water or toluene, causes gelation upon addition to the non-aqueous solution. The morphology of the gels depends on the amount of solvent: non-solvent ratio. DJ-PTCDI formed spheres that were secondarily aggregated to long fibers. MJ-PTCDI also forms vesicles that are fused together, forming honeycomb morphology, however the chaining decreased upon increasing the amount of a good solvent for Jeffamine ${ }^{\circledR}$. Hence by changing the solvents used we were able to change the gel morphology. 


\subsection{References}

1. Balakrishnan, K.; Datar, A.; Oitker, R.; Chen, H.; Zuo, J.; Zang, L. Nanobelt SelfAssembly from an Organic n-Type Semiconductor: Propoxyethyl-PTCDI. J. Am. Chem. Soc. 2005, 127, 10496-10497.

2. Balakrishnan, K.; Datar, A.; Naddo, T.; Huang, J.; Otiker, R.; Yen, M.; Zhao, J.; Zang, L. Effect of Side-Chain Substituents on Self-Assembly of Perylene Diimide Molecules: Morphology Control. J. Am. Chem. Soc. 2006, 128, 7390-7398.

3. Sinks, L. E.; Rybtchinski, B.; Iimura, M.; Jones, B. A.; Goshe, A. J.; Zuo, X.; Tiede, D. M.; Li, X.; Wasielewski, M. R. Self-assembly of Photofunctional Cylindrical Nanostructures Based on Perylene-3,4 : 9,10-bis(dicarboximide). Chem. Mater. 2005, 17, 6295-6303.

4. Gesquiere, A.; Jonkheijm, P.; Hoeben, F. J. M.; Schenning, A. P. H. J.; De Feyter, S.; De Schryver, F. C.; Meijer, E. W. 2D-Structures of Quadruple Hydrogen Bonded Oligo(p-phenylenevinylene)s on Graphite: Self-Assembly Behavior and Expression of Chirality. Nano Lett. 2004, 4, 1175-1179.

5. Schenning, A. P. H.; Herrikhuyzen, J. V.; Jonkheijm, P.; Chen, Z.; Wurthner, F.; Meijer, E. W. Photoinduced Electron Transfer in Hydrogen-bonded Oligo(pphenylene vinylene)-Perylene Bisimide Chiral Assemblies. J. Am. Chem. Soc. 2002, $124,10252-10253$.

6. Yang, X.; Xu, X.; Ji, H.F. Solvent Effect on the Self-Assembled Structure of an Amphiphilic Perylene Diimide Derivative. J Phys. Chem. B. 2008, 112, 7196. 
7. Zang, L.; Che, Y.; Moore, J.S. One-Dimensional Self-Assembly of Planar $\pi$ Conjugated Molecules: Adaptable Building Blocks for Organic Nanodevices. Acc. Chem. Res. 2008, 41, 1596.

8. Chen, Z.; Stepanenko, V.; Dehm, V.; Prins, P.; Siebbeles, L.A.; Seibt, J.; Würthner, F. Photoluminescence and Conductivity of Self-Assembled $\pi-\pi$ Stacks of Perylene Bisimide Dyes. Chem. Eur. J. 2007, 13, 436.

9. Wu, H. X.; Xue, L.; Shi, Y.; Chen, Y. L; Li, X. Y. Organogels Based on J- and HType Aggregates of Amphiphilic Perylenetetracarboxylic Diimides. Langmuir 2011, $27,3074-3082$.

10. Schmidt-Mende, L.; Fechtenkötter, A.; Müllen, K.; Moons, E.; Friend, R. H.; MacKenzie, J. D. Self-Organized Discotic Liquid Crystals for High-Efficiency Organic Photovoltaics. Science 2001, 293, 1119-1122.

11. Struijk, C. W.; Sieval, A. B.; Dakhorst, J. E. J.; Van Dijk, M.; Kimkes, P.; Koehorst, R. B. M.; Donker, H.; Schaafsma, T. J.; Picken, S. J.; vande Craats, A. M.; Warman, J. M.; Zuilhof, H.; Sudhölter, E. J. R. Liquid Crystalline Perylene Diimides: Architecture and Charge Carrier Mobilities. J. Am. Chem. Soc. 2000, 122, 11057-11066.

12. Islam, M.; Dahan, E.; Sundararajan, P.R. Preclusion of Nano Scale Self-Assembly in Block-Selective Non-Aqueous Solvents for Rod-Coil and Coil-Rod-Coil Macromolecular Surfactants Based on Perylene Tetracarboxylic Diimide. European Polymer Journal. 2012, 48(9), 1538-1554.

13. Lin, C.C.; Metters, A.T. Hydrogels in Controlled Release Formulations: Network Design and Mathematical Modeling. Adv. Drug Deliv. Rev. 2006, 58, 1379-1408. 
14. Peppas, N.A.; Bures, P.; Leobandung, W.; Ichikawa, H. Hydrogels in Pharmaceutical Formulations. Eur. J. Pharm. Biopharm. 2000, 50, 27-46.

15. Peppas, N. A.; Hilt, J. Z.; Khademhosseini, A.; Langer, R. Hydrogels in Biology and Medicine: From Molecular Principles to Bionanotechnology. Adv. Mater. 2006, 18, $1345-1360$.

16. Nuttelman, C. R.; Rice, M. A.; Rydholm, A. E.; Salinas, C. N.; Shah, D. N; Anseth, K. Macromolecular Monomers For the Synthesis of Hydrogel Niches and their Application in Cell Encapsulation and Tissue Engineering. Prog. Polym. Sci. 2008, $33,167-179$.

17. Wu, H. X.; Xue, L.; Shi, Y.; Chen, Y. L; Li, X. Y. Organogels Based on J- and HType Aggregates of Amphiphilic Perylenetetracarboxylic Diimides. Langmuir 2011, $27,3074-3082$

18. Gao, Y.; Chen, Y.; Li, R.; Bian,Y.; Li, X.; Jiang, J. Nonperipherally Octa(butyloxy)Substituted Phthalocyanine Derivatives with Good Crystallinity: Effects of MetalLigand Coordination on the Molecular Structure, Internal Structure, and Dimensions of Self-Assembled Nanostructures. Chem. Eur. J. 2009, 15, 13241-13252.

19. Gao, J.; Cheng, C.; Zhou, X.; Li, Y.; Xu, X.; Du , X.; Zhang, H. Synthesis of Size Controllable cu-phthalocyanine Nanofibers by Simple Solvent Diffusion Method and their Electrochemical Properties. Journal of Colloid and Interface Science 2010, 342, $225-228$.

20. Dahan, E.; Sundararajan, P.R. Thermo-reversible Gelation of Rod-Coil and Coil-RodCoil Molecules Based on Poly (Dimethyl Siloxane) and Perylene Imides without Hydrogen Bonding Groups. Submitted to Langmuir 2013 
21. Brandrup, J. (Johannes) Polymer Handbook Chichester, Wiley: New York, 1999.

22. Li, Y.; Ma, Q.; Huang, C.; Liu, G. Crystallization of Poly (ethylene glycol) in Poly (methyl methacrylate) Networks. Materials Science 2013, 19(2), 147-151.

23. Kasha, M.; Rawls, H. R.; El-Bayoumi, M. A. The Exciton Model in Molecular Spectroscopy. Pure Appl. Chem. 1965, 11, 371-372.

24. Clark, A. E.; Qin, C.; Li, A. D. Beyond Exciton Theory: A Time-Dependent DFT and Franck-Condon Study of Perylene Diimide and its Chromophoric Dimer. J. Am. Chem. Soc. 2007, 129, 7586-7595.

25. Wang, W.; Han, J. J.; Wang, L. Q.; Li, L. S.; Shaw, W. J.; Li, A. D. Dynamic $\pi-\pi$ Stacked Molecular Assemblies Emit from Green to Red Colors. Nano Lett. 2003, 3, 455-458.

26. Qintang Li,Q.; Wang,X.; Yuea, X.; Chen, X. Wormlike Micelles Formed Using Gemini Surfactants with Quaternary Hydroxyethyl Methylammonium Head Groups.

Soft Matter. 2013, 9, 9667-9674 


\section{CHAPTER 6}

\section{Gelation of Oligostyrene Functionalized Perylene Diimide}

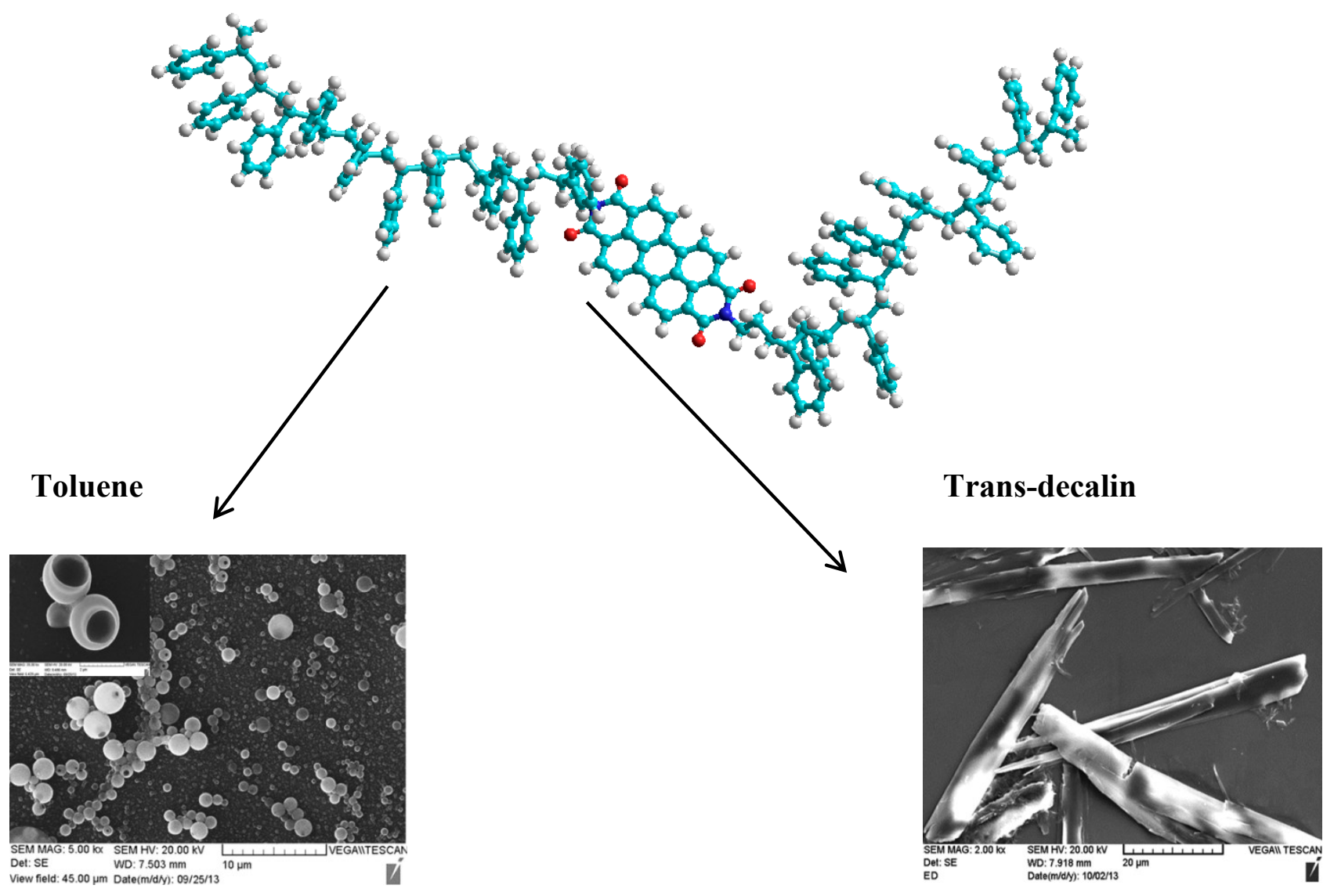




\subsection{Introduction}

Polystyrene is one of the polymers, the gelation of which was studied by several authors over three decades ago. These studies were aimed towards understanding the mechanism of gelation of polymers. While the phenomenon is generally attributed to liquid-liquid phase segregation arrested by vitrification, there is a further dimension to this due to the tacticity of the polymer. In the case of isotactic polystyrene (i-PS), phase separation would be caused by crystallization as well. Lemstra and Challa ${ }^{1}$ reported the crystallization of i-PS from dilute solutions. Wellinghoff et. al. ${ }^{2}$ studied the gelation of isotactic and atactic polystyrenes (a-PS) from various solvents, for the purpose of preparing glassy polymer films via the gelation route. They found that the glassy film from i-PS in fact consisted of microcrystalline regions. X-ray diffraction studies of Atkins et. al. ${ }^{3}$, Girolamo et. al. ${ }^{4}$, and Sundararajan et. al. ${ }^{5}$ indicated that even the conformation of the i-PS chain depended on the type of solvent used for the gelation experiment. Sundararajan et. al. ${ }^{5}$, showed that the conformation of the chain in the gels using aromatic vs aliphatic solvents differed. Guenet et. al. ${ }^{6}$ argued that the differences in the X-ray diffraction profiles could be attributed to complex formation of the solvent with i-PS chains. The fact that crystallization of the chain is not a requirement was established by gelation studies on aPS. It was demonstrated by Hikmet et. al. ${ }^{7}$ that thermo-reversible gelation of atactic polystyrene (a-PS) with cyclohexanol as a solvent, is the consequence of liquid-liquid phase segregation arrested by vitrification. The gel setting temperature was found to be close to $50^{\circ} \mathrm{C}$, and the morphology was described as molecularly connected glassy beads in a solvated matrix.

Oligostyrene is widely used in studies of block copolymer self-assembly ${ }^{8-13}$. These consisted usually of highly asymmetric blocks, with one of the blocks being water soluble. Eisenberg et. al. pioneered the research on such amphiphilic block copolymers. For example, 
depending on the relative length of the blocks in polystyrene-b-poly (acrylic acid), morphologies ranging from micelles to vesicles to worm-like structures have been reported. Deepak and Sundararajan ${ }^{14}$ attached an oligostyrene segment (with a $\mathrm{T}_{\mathrm{g}}$ of $43^{\circ} \mathrm{C}$ ) to terthiophene, and the self-assembly of the terthiophene segment led to solid or hollow spheres depending on the solvent composition. In the present work, we attached oligostyrene to both sides of the PTCDI (PS-PTCDI-PS), to form a coil-rod-coil molecule, and studied its gelation behavior. This differs from the case of coil-rod-coil molecules discussed in Chapters 4 and 5. With Di-PDMS, the $T_{g}$ of the PDMS segment is $-125{ }^{\circ} \mathrm{C}$. With DJ-PTCDI, considering that the glass transition temperatures of PEO and PPO are -50 and $-75{ }^{\circ} \mathrm{C}$, respectively, the $\mathrm{T}_{\mathrm{g}}$ of Jeffamine can be expected to be about $-60{ }^{\circ} \mathrm{C}$. However, the oligostyrene segment that we used here has a $\mathrm{T}_{\mathrm{g}}$ of 43 ${ }^{\circ} \mathrm{C}$. Further, while the rod segment is a large aromatic chromophore, and the oligostyrene coil has aromatic side groups (Scheme 6.1). Previous studies of Islam and Sundararajan ${ }^{15}$ on this molecule in solution showed that self-assembly occurred only in solvent mixtures.

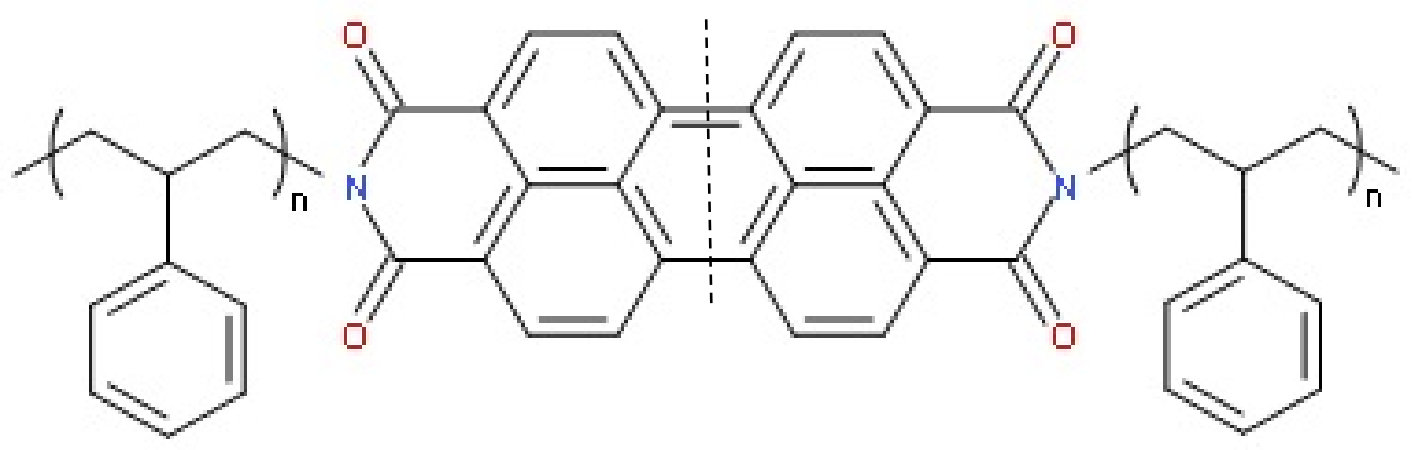

Scheme 6.1 Structure of PS-PTCDI-PS block co-polymer used the gelator. Dashed line is to illustrate symmetry of the block co-polymer

In this chapter, we discuss the effect of the oligostyrene coil on the self-assembly of PTCDI in block selective solvents as well as the conditions under which self-assembly and hence 
gelation occurs. Since aPS has a planar $\pi$-bonding side group, we investigated whether additional support to the $\pi-\pi$ interaction of the perylene units would enhance aggregation and hence gelation. This chapter describes the gelation properties as well as the resulting morphology of the extended $\pi$-electron system of a-PS. We show here how the unique combination of a flexible polymer and a rigid, extended, $\pi$-electron system (PS-PTCDI-PS) could lead to interesting morphologies.

\subsection{Experimental}

To synthesize PS-PTCDI-PS, 3, 4, 9, 10-Perylenetrtracarboxylic acid dianhydride, dimethyl acetamide (DMAc) and zinc acetate dihydrate were purchased from Aldrich Chemical Company and used as received. Amine terminated polystyrene (average molecular weight 1200) was purchased from Polymer Source Inc. and used as received. The synthetic procedure was described by Islam and Sundararajan, ${ }^{15}$ and is given in Appendix 3. For the gelation studies, the solvents used were purchased from Aldrich Chemical Company Inc. PS-PTCDI-PS gels of different concentrations $(3.00-10.0 \mathrm{mM})$ were prepared by dissolving the solutes in the required amount of cyclohexane, toluene, or trans-decalin at temperatures ranging from $78-83{ }^{\circ} \mathrm{C}$ with constant stirring. This resulted in a red solution. Closed vials were used to avoid the evaporation of the solvent. The gels were prepared by slow cooling the solution and gelation was confirmed by tube inversion, i.e., no flowing solvent. Xerogels were prepared by drying the gels at room temperature for a few days and then under vacuum overnight for complete removal of solvents. 


\subsection{Results and Discussion}

Figure 6.1 shows the effect of concentration on the temperatures of dissolution, onset of gelation and completion of gelation, respectively. For example with the gels that were formed with cyclohexane at $8.0 \mathrm{mM}$ concentration (Figure 6.1 (a)), a red solution was obtained at $78{ }^{\circ} \mathrm{C}$. When the solution was slow cooled, a deep red-colored, opaque and immobile gel began to form at $47^{\circ} \mathrm{C}$. It is seen that the onset of gelation differs from one solvent to the other, for example, the on-set of gelation with cyclohexane is $47^{\circ} \mathrm{C}$ at $8.00 \mathrm{mM}$ while for the gels formed with trans-decalin, it is higher, at $53^{\circ} \mathrm{C}$. This could be due to the different packing of the molecules, which ultimately leads to a different morphology. In addition, the minimum gel concentration with cyclohexane and toluene is $5.00 \mathrm{mM}$ while that of trans-decalin is $3.00 \mathrm{mM}$; which confirms that the form of aggregation is different when using the different solvents. It was mentioned above that the gelation of isotactic or atactic polystyrene (the polymer by, without the perylene diimide as used here) was influenced by the type of solvent.

DSC measurements for the different solvents not only confirm the reversibility of the gels but also, the variation of the gelation temperature with the different solvents. Figure 6.2 (a) shows the DSC trace of the gels formed with cyclohexane with an endotherm at $47^{\circ} \mathrm{C}$ in the first heating cycle corresponding to the gel-sol transition, and a sol-gel transition exotherm at $41^{\circ} \mathrm{C}$ upon cooling, and a gel-sol transition during the second heating cycle. This confirms the thermoreversibility of the gels. Figure 6.2 (b) shows the DSC trace of the gels formed with trans-decalin with an endotherm at $52{ }^{\circ} \mathrm{C}$ in the first heating cycle corresponding to the gel-sol transition, and a sol-gel transition exotherm at $46^{\circ} \mathrm{C}$ upon cooling, and a gel-sol transition during the second heating cycle. This confirms that the onset of gelation is higher for trans-decalin than the other two solvents, which may be due to the difference in the molecular packing. 

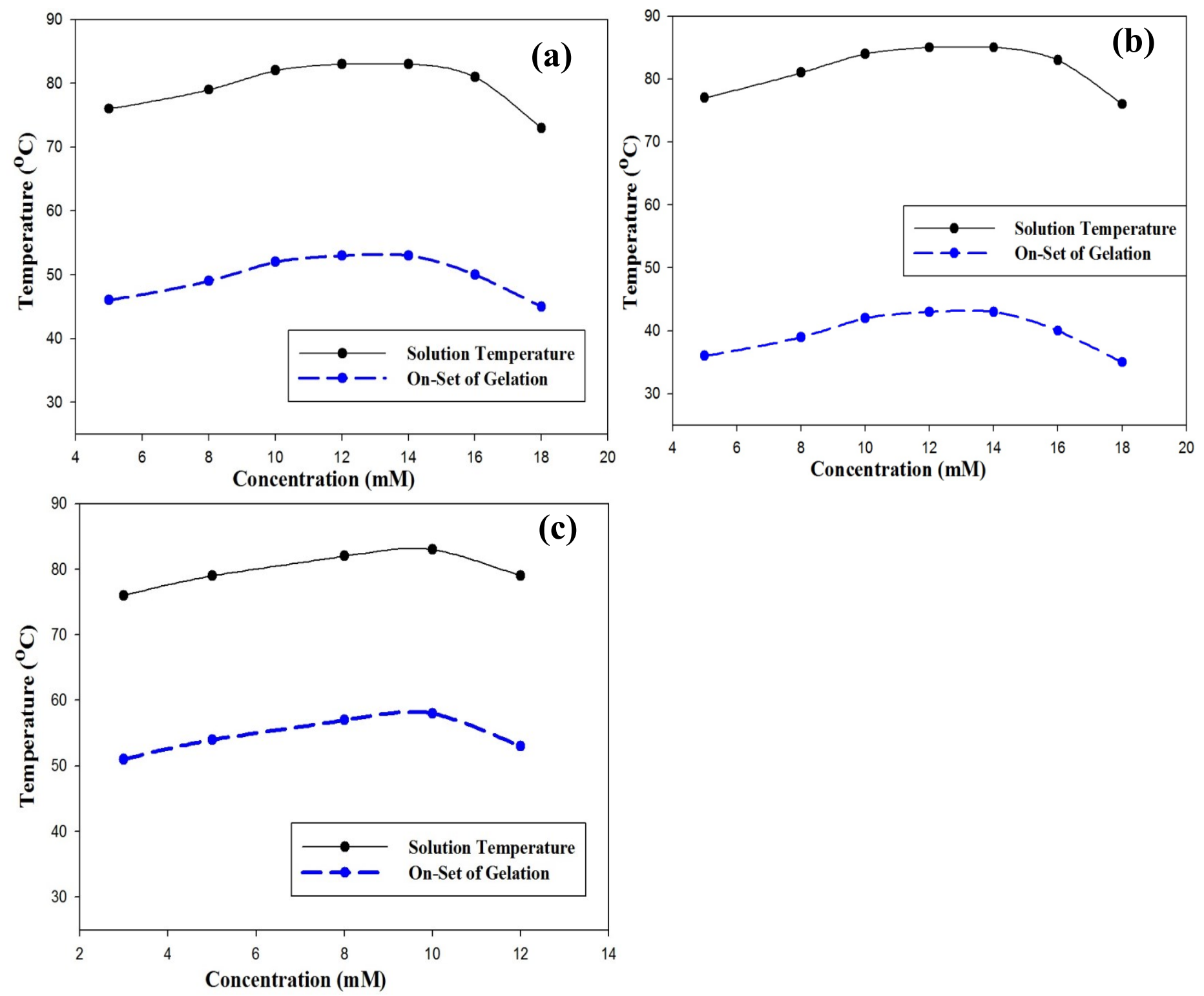

Figure 6.1 Phase diagrams of gels formed with (a) cyclohexane (b) toluene (c) trans-decalin 

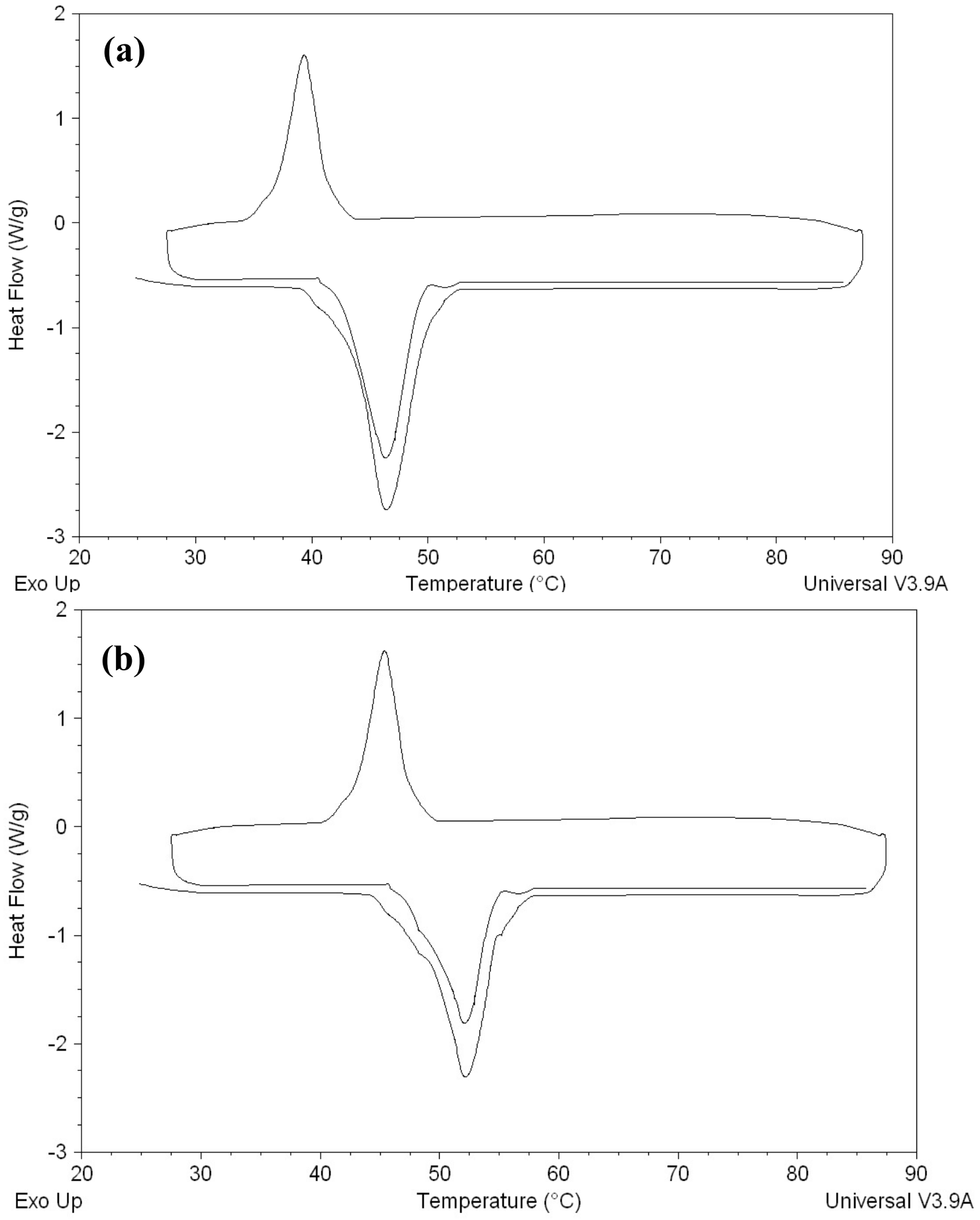

Figure 6.2 DSC traces of $8.00 \mathrm{mM}$ Gels in (a) cyclohexane (b) trans-decalin 


\subsubsection{Morphology}

In this work, the short hydrophobic polystyrene is used as the peripheral side chain. Polystyrene has a planar $\pi$ bonding moiety that may give additional support to the $\pi-\pi$ interaction of the perylene units. Different morphologies varying from hollow spheres to folded fibers are seen, depending on the solvents used. As seen in the SEM and TEM images (Figure 6.3), when toluene and cyclohexane were used as the solvents, a spherical morphology was obtained; however when trans-decalin was used as the solvent, fibers are seen, with what appears to be folded as eaves trough. The fibers are a few hundred microns in length. What can also be observed is that the fibers become denser with an increase in concentration.

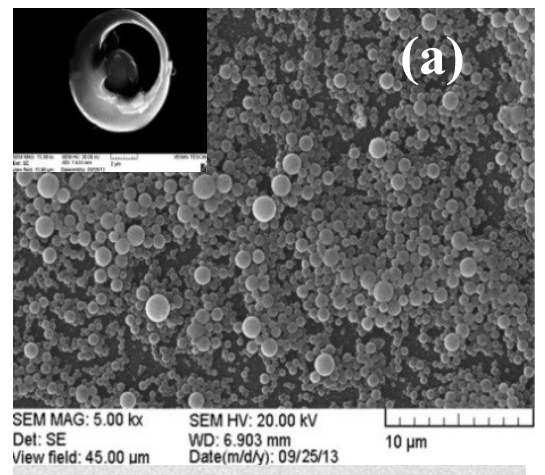

(d)

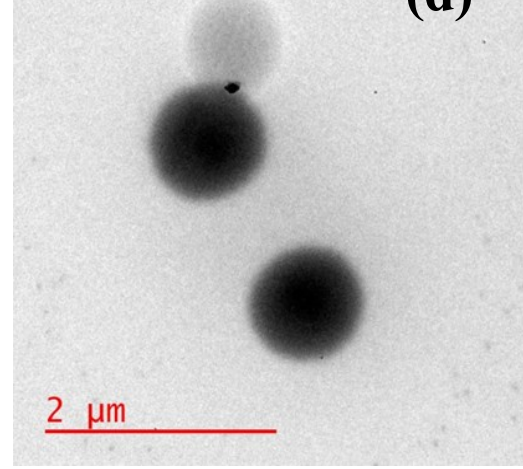

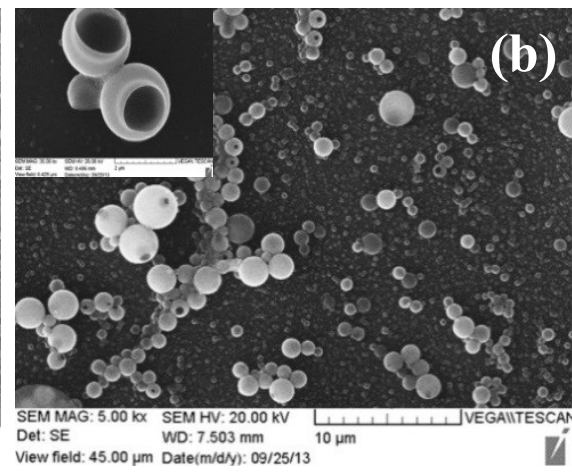

(e)

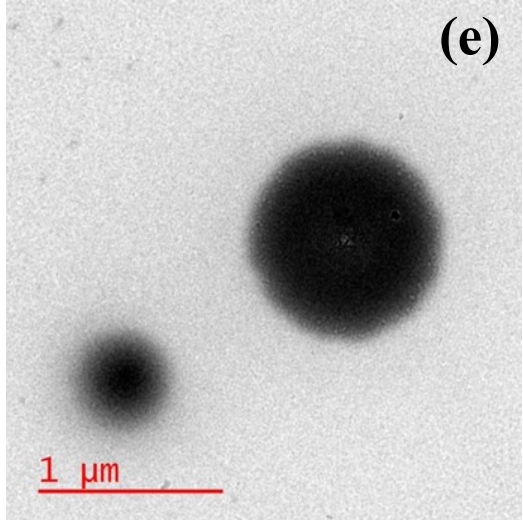

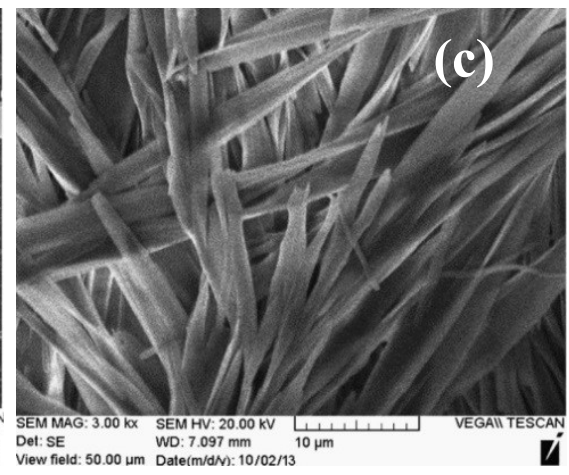

(f)

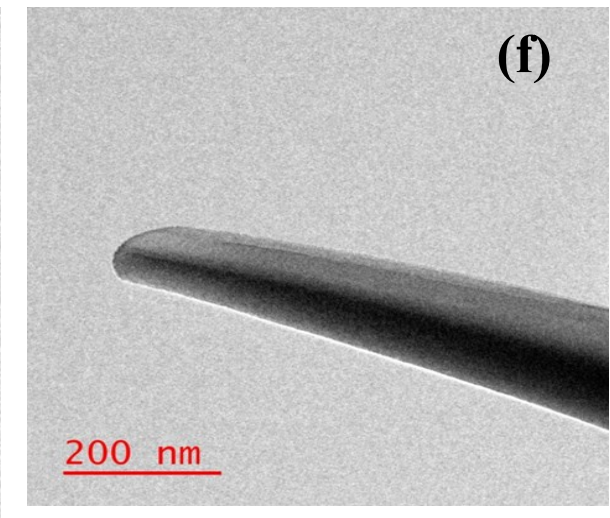

Figure 6.3 SEM of Xerogels from (a) Cyclohexane (b) Toluene (c) trans-decalin; and TEM of

Xerogels from (d) Cyclohexane (e) Toluene (f) trans-decalin 
However when the concentration of the gelator increases to and beyond $10.00 \mathrm{mM}$, part of the sample starts to crystallize instead of forming a gel (Figure 6.4 (d), (e)). In a previous work by De Rudder et. al. ${ }^{16}$ it was found that in solutions of syndiotactic polystyrene in cisdecalin there is a competition between crystallization and gelation. The molecular conformation that is adopted by the polymer chain depends on the annealing temperature. At low temperature the helix conformation is kinetically favored and a gel is formed; however, between 45 and $70^{\circ} \mathrm{C}$ the zigzag conformation prevails and limited crystallinity is obtained ${ }^{9}$. However, in the case of our work, the perylene segment is attached to atactic polystyrene. Since the atactic polystyrene (a-PS) segment is totally uncrystallizable, yet the gelator was found to gel trans-decalin at lower concentrations, crystallization is attributed to the packing of the perylene segment. 

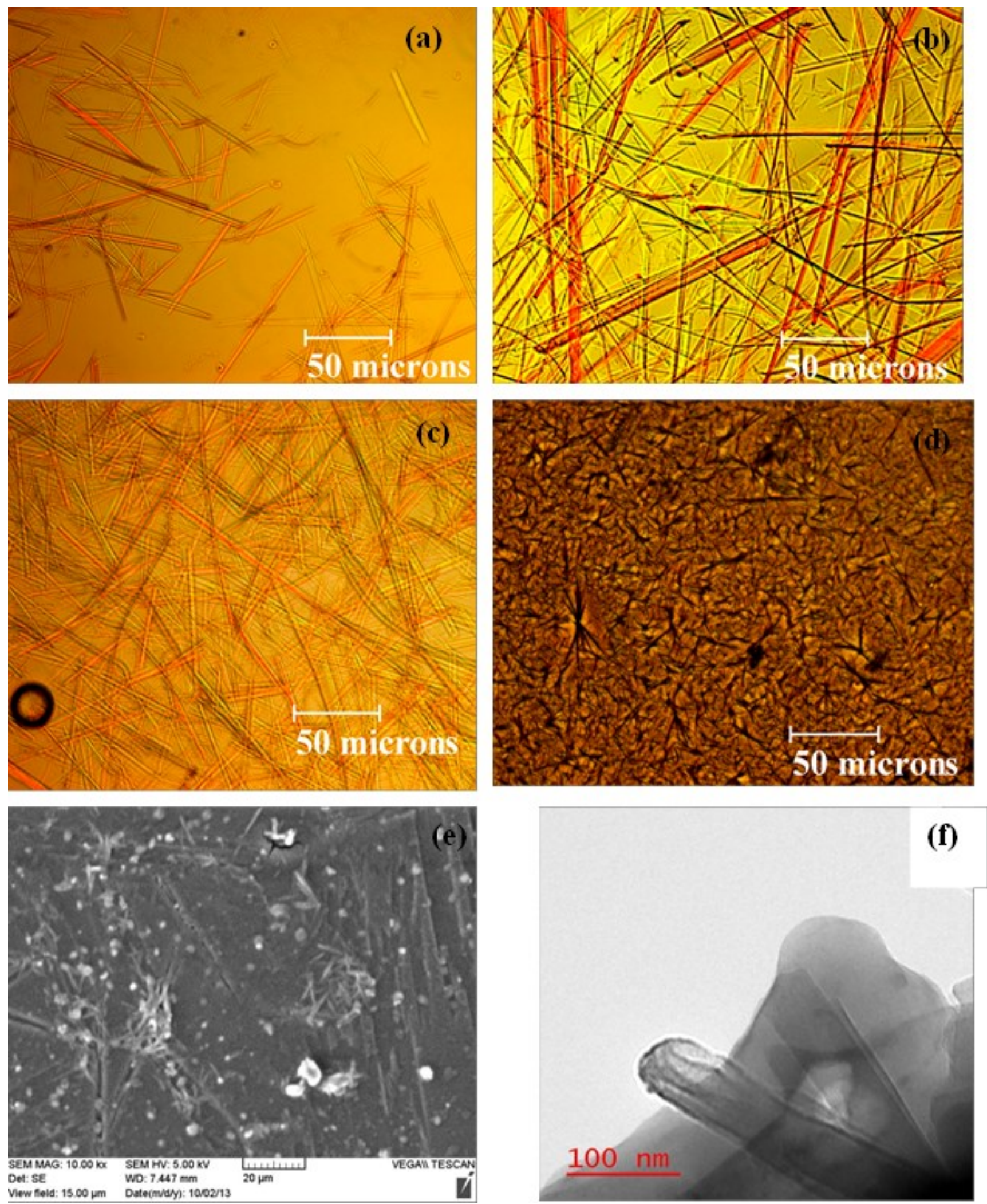

Figure 6.4 OM of Gels in trans decalin at: (a) 3.00 (b) 5.00 (c) 8.00 (d) $10.00 \mathrm{mM}$; (e) SEM and

(f) TEM of Xerogel at $10.00 \mathrm{mM}$ 


\subsubsection{Optical Properties}

In our previous studies ${ }^{15}$, on the self-assembly of PS-PTCDI-PS, in various solvents, we found that the monomeric form of this compound in chloroform showed three distinct peaks in UV-Vis absorption studies. The peak positions originating from S0-2, S0-1 and S0-0 vibronic transitions are at 456, 489 and $526 \mathrm{~nm}$ respectively. In our present gelation studies, we found that the UV-Vis spectra show only two peaks that red shifted relative to the monomeric form. Due to the presence of the short chain atactic PS chain, complete face to face overlap between two successive perylene units is distorted and a transverse stacking becomes stable. Figure 6.5 (a) is a UV-Vis spectrum of $10.00 \mathrm{mM}$ gels prepared with toluene, cyclohexane or trans-decalin. With toluene, the major peak is at $501 \mathrm{~nm}$, a second peak is at $536 \mathrm{~nm}$ and they have an intensity ratio of 1: 0.85 . With cyclohexane, the major peak is at $504 \mathrm{~nm}$, the second peak is $540 \mathrm{~nm}$, and their relative intensity ratio is $1: 0.94$. With trans-decalin, the major peak is at $502 \mathrm{~nm}$, a second peak is at $547 \mathrm{~nm}$ and their intensity ratio is 1:0.49. This difference in intensity and slight blue shift from the other solvents used, may be due to the difference in packing of the PTCDI, and hence the difference in morphology as seen in the other sections. The intensity of the peak at $547 \mathrm{~nm}$, increases at lower gel concentration (Figure 6.5 (b)). The fluorescence spectrum shows fluorescence quenching with a slight red shift from $539 \mathrm{~nm}$ to $543 \mathrm{~nm}$ when increasing the concentration from $3.00 \mathrm{mM}$ to $10.00 \mathrm{mM}$ (Figure 6.5 (c)). At higher concentration, more aggregation of PTCDI occurs and the polystyrene segment is also a collapsed state hence the fluorescence quenching. 

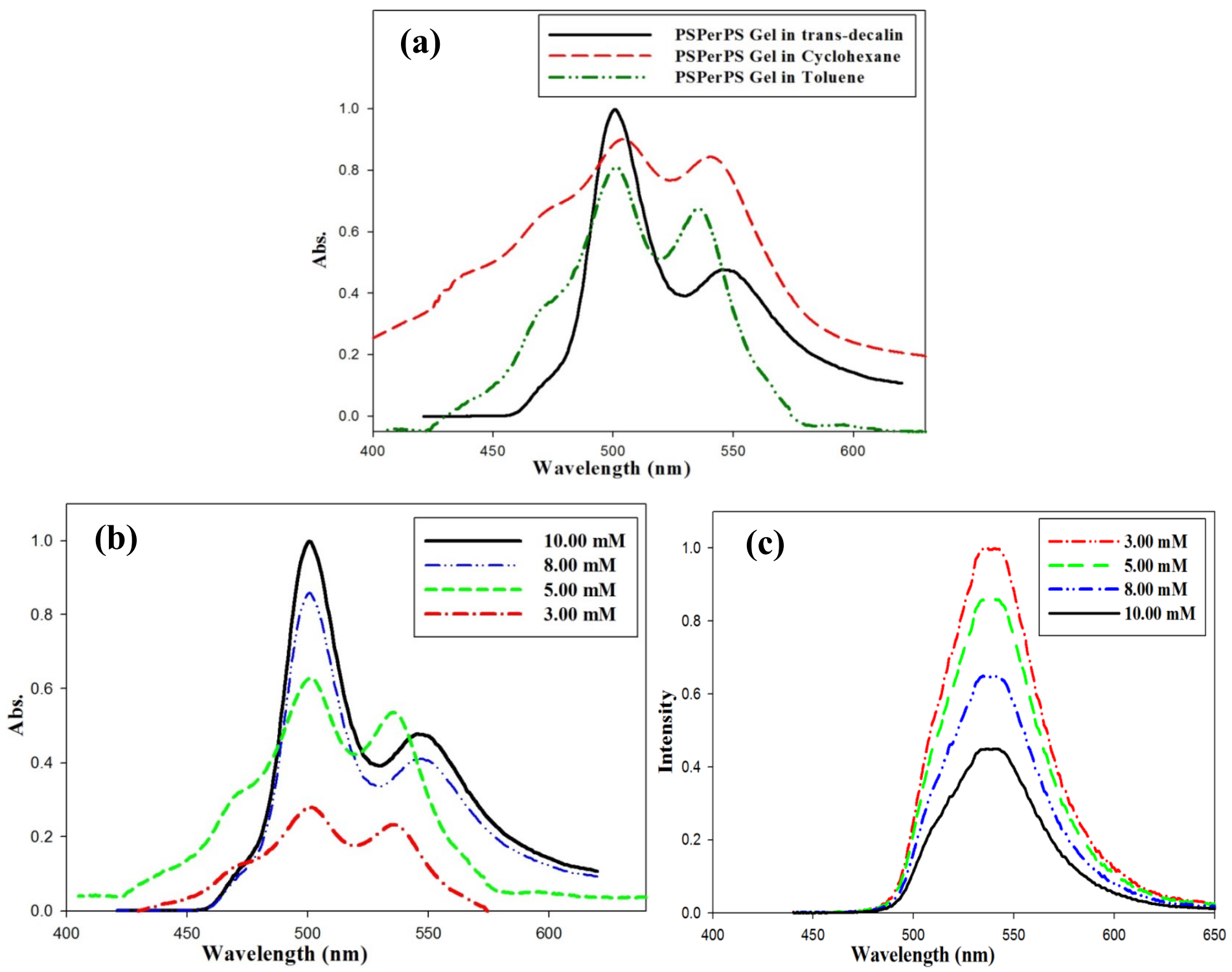

Figure 6.5 (a) UV-Vis of $10.00 \mathrm{mM}$ PSPerPS Gel from different solutions; (b) UV-Vis and

(c) Fluorescence Intensity of PSPerPS Gel from trans- decalin 


\subsubsection{XRD Studies}

PS-PTCDI-PS shows diffraction peaks at 8.03, 6.34, 3.36 and 3.05 , and $2.91 \AA$ confirming the aggregation of the perylene segment in cyclohexane (Figure 6.6 (a)). In transdecalin (Figure 6.6 (b)), we see additional peaks and the peaks shift to larger 2-theta values, due to the difference in packing of the perylene segments and the fact that fibers were obtained as opposed to vesicles, when trans decalin was used as the solvent. The large hump (amorphous scattering) at about $2 \theta=20-25^{\circ}$ is due to the atactic polystyrene segment. With very few reflections, it is difficult to make any conclusions with regard to the packing of the molecules, except to say that the diffraction profiles of the gels from the two solvents and hence the packing of the molecules are different. 


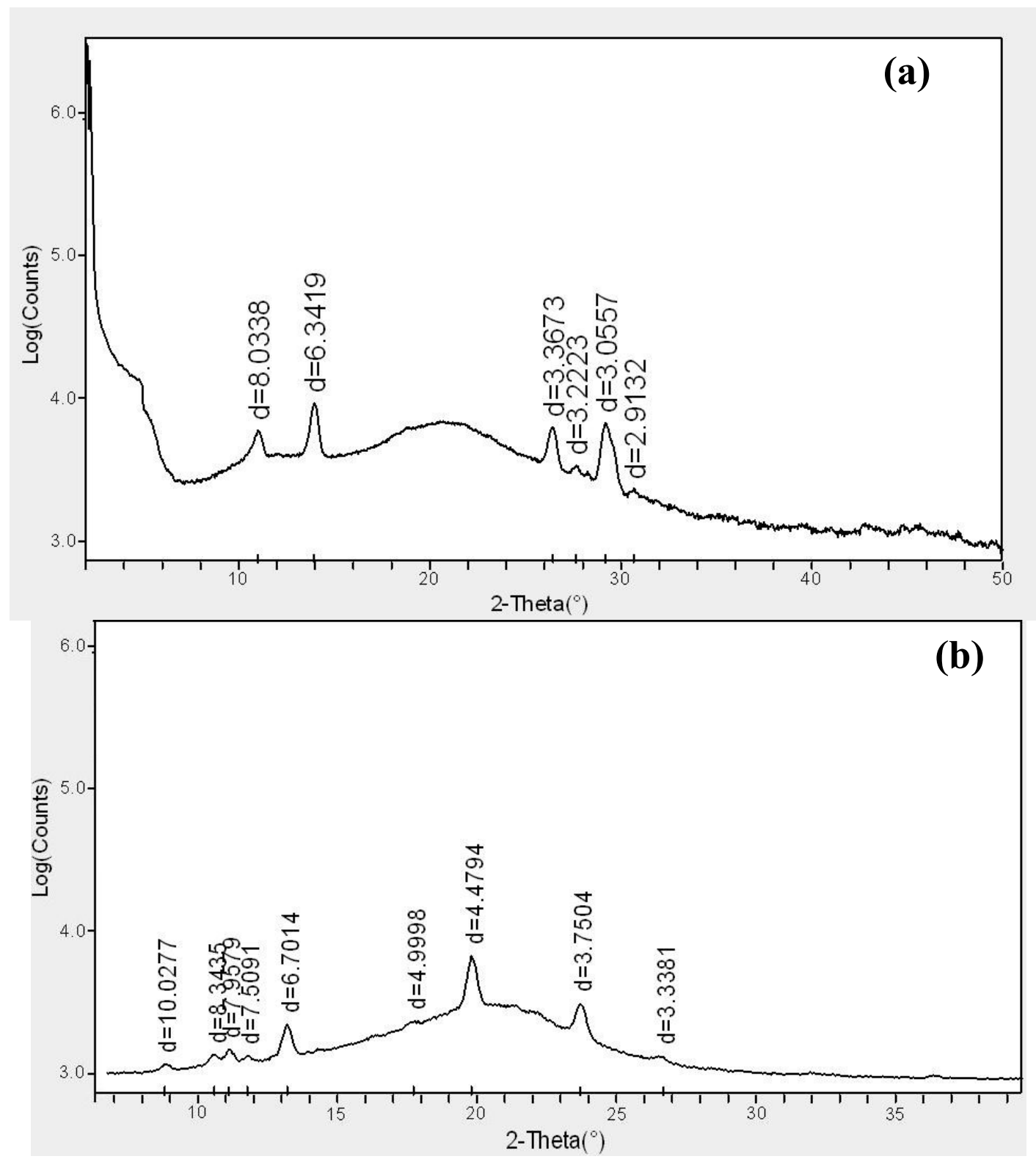

Figure 6.6 XRD traces of PS-PTCDI-PS Xerogels from (a) cyclohexane (b) trans-decalin 


\subsubsection{Rheology}

It is seen that for all the PS-PTCDI-PS gels, the $G^{\prime}$ is higher than the G" and remains almost invariant through the frequency range. This confirms that all are indeed gels. The gels from trans-decalin (Figure 6.7 (c)) show higher moduli than the gels from cyclohexane (Figure 6.7 (a)) or toluene (Figure 6.7 (b)) and in this case also the $G^{\prime}$ is higher. This may be due to the morphology of these gels which consists of long fibers, with possible entanglements as opposed to spheres. If these gels were compared to the DJ-PTCDI gels in chapter 5 (Figure 5.9), it can be

seen that PS-PTCDI-PS gels have a higher G'. This is possibly attributed to the high $\mathrm{T}_{\mathrm{g}}{ }^{10}\left(\mathrm{~T}_{\mathrm{g}}=\right.$ $43^{\circ} \mathrm{C}$ ) of the amino terminated oligostyrene that was used to synthesize PS-PTCDI-PS; which can contribute to a tougher gel. 

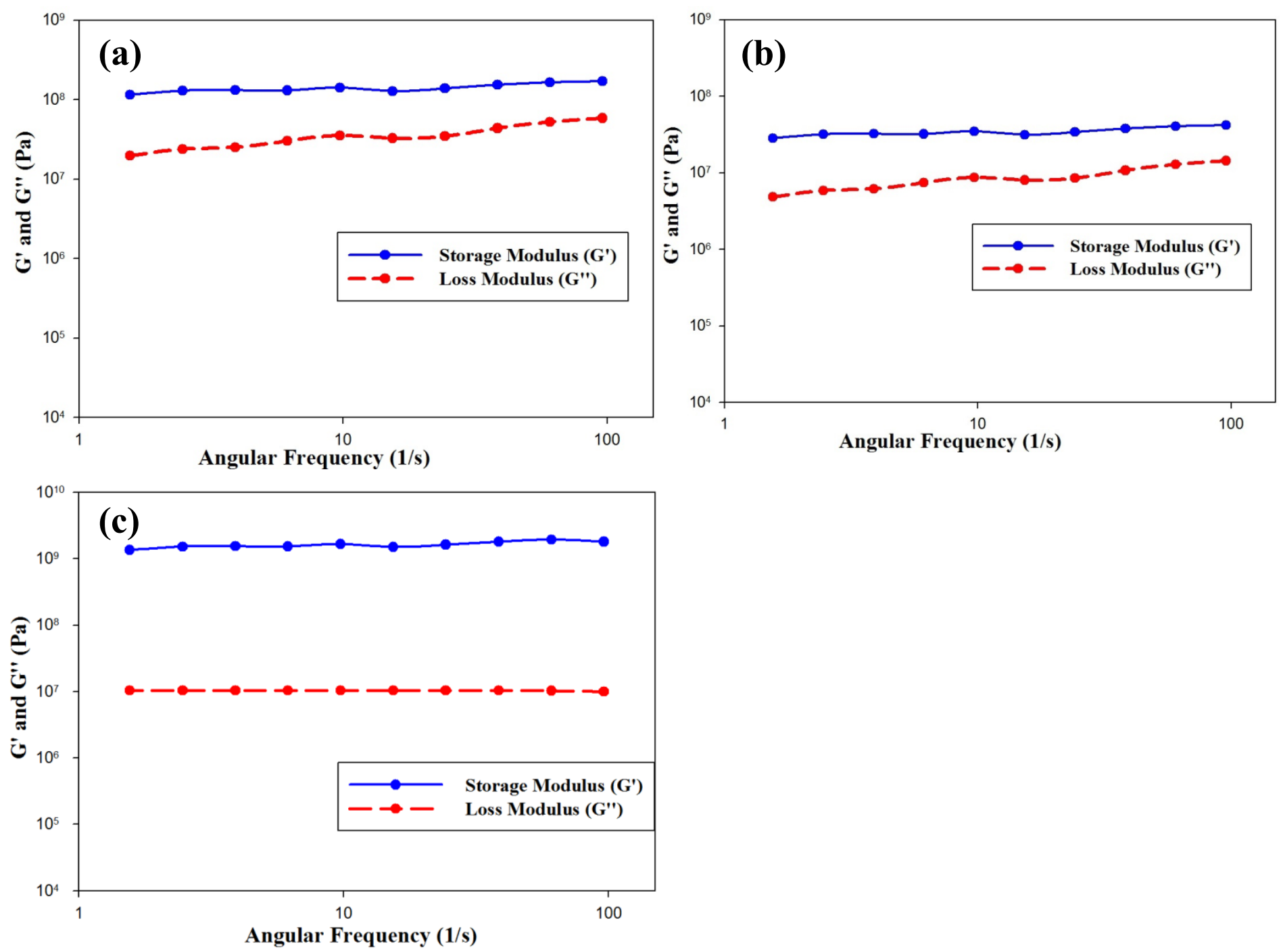

Figure 6.7 Variation of G' and G" with frequency of PS-PTCDI-PS gels with (a) cyclohexane (b) toluene (c) trans-decalin 


\subsection{Conclusions}

This chapter describes the gelation properties as well as the resulting morphology of the extended $\pi$-electron system of PS-PTCDI-PS. We show here how the unique combination of a flexible polymer (a-PS) and a rigid, extended, $\pi$-electron system (PTCDI) could lead to interesting morphologies. When cyclohexane and toluene were used as the solvents, spherical morphologies were obtained; while fiber like morphology was obtained when trans-decalin was used. At higher concentration of the gelator, more aggregation of PTCDI occurs and the polystyrene segment is also a collapsed state hence crystallization may be observed. 


\subsection{References}

1. Lemstra, P. J.; Challa, G. J. Crystallization of Isotactic Polystyrene from Dilute Solutions. Polym. Sci., Polym. Phys. Ed. 1975, 13, 1809.

2. Wellinghoff, S.; Shaw J.; Baer, E. Polymeric Materials from the Gel State. The Development of Fringed Micelle Structure in a Glass. Macromolecules 1979, 12, 932.

3. Atkins, E.D.T.; Isaac, D.H.; Keller, A. Conformation of Polystyrene with Special Emphasis to the Near All-Trans Extended-Chain Model Relevant in Polystyrene Gels $J$. Polym. Sci., Polym. Phys. Ed. 1980, 18, 71.

4. Girolamo, M.; Keller, A.; Miyasaka, K.; Overbergh, N. Gelation-Crystallization in Isotactic Polystyrene Solutions and its Implications to Crystal Morphology, to the Origin and Structure of Gels, and to the Chemical Homogeneity of Polyolefins. J. Polym. Sci. Polym. Phys. Ed. 1976, 14, 39.

5. Sundararajan, P.R.; Tyrer, N.; Bluhm, T. Solvent Dependent Conformations in Gels of Isotactic Polystyrene. Macromolecules, 15, 286-90 (1982).

6. Guenet, J.M.; Lotz, B.; Wittmann, J.C. Thermodynamic Aspects and Morphology of Physical Gels from Isotactic Polystyrene. Macromolecules 1985, 18, 420-427.

7. Hikmet, R.M.; Callister, S.; Keller, A. Thermo-reversible Gelation of Atactic Polystyrene: Phase Transformation and Morphology. Polymer 1988, 29, 1378-1388.

8. Burke, S.; Shen, H.; Eisenberg, A. Multiple Vesicular Morphologies from Block Copolymers in Solution. Macromol. Symp. 2001, 175, 273-283.

9. Discher, D.; Eisenberg, A. Polymer Vesicles Science 2002, 297, 967-973. 
10. Yu, K.; Eisenberg, A. Multiple Morphologies in Aqueous Solutions of Aggregates of Polystyrene-block-Poly(ethylene oxide) Diblock Copolymers. Macromolecules 1996, 29, 6359-6361.

11. Zhang, L.; Eisenberg, A. Morphogenic Effect of Added Ions on Crew-Cut Aggregates of Polystyrene-b-poly(acrylic acid) Block Copolymers in Solutions. Macromolecules 1996, 29, 8805- 8815 .

12. Zhang, L.; Eisenberg, A. Multiple Morphologies of "Crew-Cut" Aggregates of Polystyrene-b-Poly(acrylic acid) Block Copolymers $J$. Am. Chem. Soc. 1996, 118, 31683181.

13. Yu, K.; Eisenberg, A. Bilayer Morphologies of Self-Assembled Crew-Cut Aggregates of Amphiphilic PS-b-PEO Diblock Copolymers in Solution. Macromolecules 1998, 31, 3509-3518.

14. Deepak, V.; Sundararajan, P.R. Solvent Mixture Induced Self Assembly of a Terthiophene Based RodCoil Block Co-oligomer. J. Phys. Chem. B. 2011, 115, 84588464.

15. Islam, ,M.; Sundararajan, P.R. Nano-Scale Self-Assembly Impeded by $\mathrm{CH} \cdots \pi$ Interaction in Block Selective Solvents in the Case of Oligostyrene-Perylene diimide-Oligostyrene (Coil-Rod-Coil) Molecule. European Polymer Journal 2013, 49, 2042-2051.

16. De Rudder, J.; Berge, B.; Berghmans, H. Competition between Gelation and Crystallization in Solutions of Syndiotactic Polystyrene in cis-Decalin. Macromol. Chem. Phys. 2002, 203, 2083-2088. 


\section{CHAPTER 7}

\section{The Fabrication of Composite Films of Self-Assembled PS-PTCDI-PS in a Polymer Matrix}

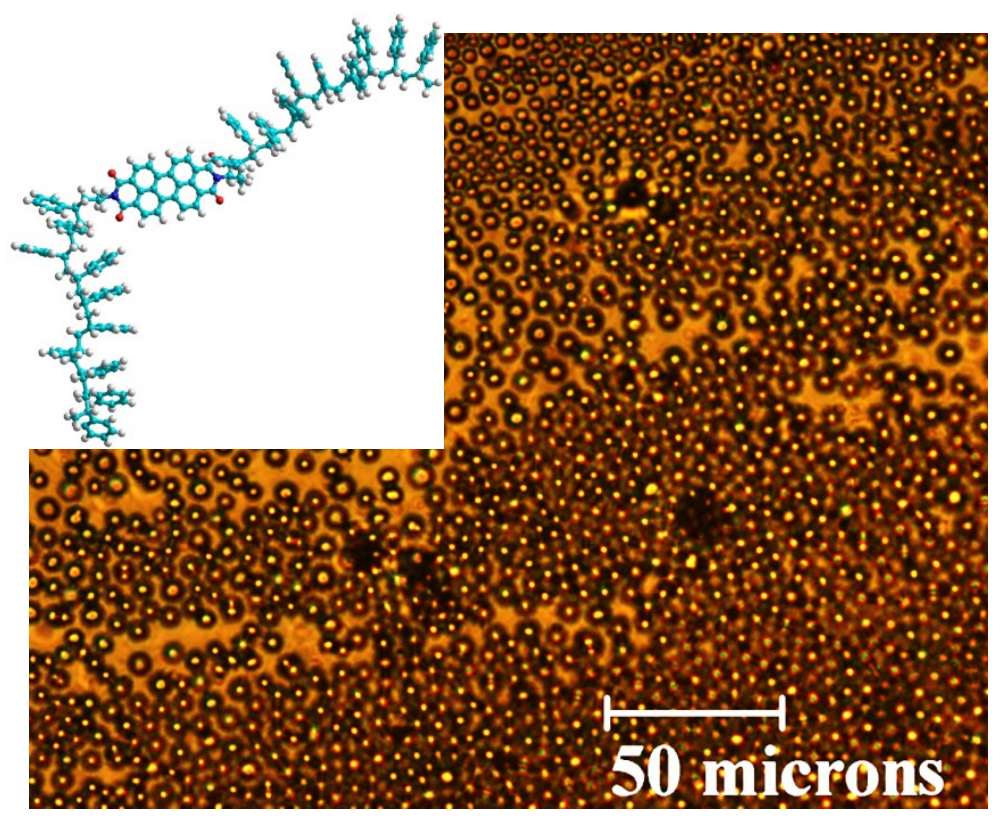




\subsection{Introduction}

Polymers doped with small molecules have a wide range of applications in organoelectronic devices such as photoreceptors, organic light emitting diodes (OLED) and electrochromic devices. These are multi-layer composites, generally prepared as a double solid solution, consisting of charge transporting donor or acceptor molecules dispersed in a polymer binder $^{1,2}$. Several authors have proposed that the charge transport occurs by the hopping mechanism, and this in turn depends on the dispersion of the active molecule and the intermolecular distance between these molecules ${ }^{2-4}$. Any phase separation of the active molecule would lead to its crystallization and deterioration of the device performance. Controlling the phase separation in complex systems such as polymer-small molecule mixtures to render functional morphologies has been an active area of research. For example, Santerre et al. ${ }^{5}$ reported the properties of OLED devices based on N, N'-diphenyl-N, N'-bis (3-methylphenyl)[1, 1'-biphenyl]-4, 4'-diamine (TPD) dispersed in polymers with high $\mathrm{T}_{\mathrm{g}}$. The best performance was obtained with a TPD concentration of $75 \%$. Our group previously studied ${ }^{6}$ binary solid solutions of TPD in bisphenol A polycarbonate (BPAPC) and cyclohexyl polycarbonate (PCZ) with three different molecular weights. It was shown that molecularly doped TPD in polymers significantly depressed the $\mathrm{T}_{\mathrm{g}}$ of the host polymers. It was also noted that lower degree of crystallinity was observed in the case of TPD/PCZ, which is due to the lower molecular flexibility of the PCZ relative to BPAPC ${ }^{6}$. Hence the molecular structures and the conformational flexibility of the host and guest, plays a role in determining the compatibility of the system. However, no specific molecular level interaction, other than a weak van der Waals force, exists between these functional small molecules and the host polymer matrix. 
In another study ${ }^{7}$, Tuteja et. al. described a polymer dispersed self-assembling small molecule system, in which a homologous series of carbamates, with a hydrogen-bonding moiety and alkyl side chains, was dispersed in polycarbonate. The self-assembling molecules form colloidal size domains in the polymer, and this involves a hierarchy of three levels of assembly. The molecules self-assemble into small crystallites, which then organize into spherulitic structures. These spherulites then aggregate to form large, uniform near-spherical domains. The size and uniformity of the domains depend on the length of the alkyl side chain ${ }^{7}$. We anticipated that similar domain formation with other self-assembling systems may be obtained.

In the design of optoelectronic devices, there is an emphasis on flexible configurations. For example, the electric paper should be flexible similar to a regular paper, so that it could be rolled during handling. For such flexible devices, functional small molecules by themselves are not sufficient because they do not form strong films. The small molecule has to be dispersed in a polymer matrix and even then, phase separation could occur. When perylene by itself was dispersed in a polycarbonate matrix, the small molecule formed discrete crystals of a few microns, irrespective of the solvent used for casting the films (Islam and Sundararajan, unpublished work). We believed that by attaching a side group which is compatible with a polymer, the mixing would be better, and a molecularly dispersed composite film could be obtained. For example, with oligostyrene attached to PTCDI, we expected that a better dispersion in polystyrene could be achieved. In this chapter, we disperse PS-PTCDI-PS in their corresponding polymer, polystyrene (PS), in an attempt to make composite polymer films. Further research on these composite polymer films will shed light on their outstanding optical properties due to the presence of the perylene units. 


\subsection{Experimental}

The synthetic route of PS-PCTDI-PS is given in Appendix 3. To cast the films, a high molecular weight polystyrene $\left(M_{w}=239,700\right.$ and $\left.M_{n}=119,600\right)$, was purchased from Aldrich Chemical Company Inc. For most of the experiments, PS-PCTDI-PS concentrations of 1, 5, 10 $\mathrm{wt} \%$ in PS were used. Solvent cast films were prepared by dissolving appropriate mixtures of the PS-PTCDI-PS and polymer in chlorobenzene, THF, or chloroform of laboratory grade. The solvents used were purchased from Aldrich Chemical Company Inc. Films were coated on a glass substrate using an electrically driven film coater and were dried at very low rate of the solvent evaporation at ambient conditions for $24 \mathrm{~h}$ and then under vacuum for $24 \mathrm{~h}$. The final thickness of the films was about $20 \mu \mathrm{m}$.

\subsection{Results and Discussion}

\subsubsection{Morphology}

Even though a high concentration of the charge transport molecule is preferred to improve the overall sensitivity of the device, this must be accomplished without phase separation or crystallization from the polymer matrix. The work described in this Chapter is preliminary and future work would examine the bahaviour of these composites in detail. Figure 7.1 shows the optical micrographs of the films prepared with various solvents. The films that were prepared with chlorobenzene and chloroform showed spherical domains $(10-20 \mu \mathrm{m})$, that were embedded in the polymer matrix as observed in Figure 7.1 (a), (b), (d), (e) and Figure 7.2 (a), (b). This could be attributed to the self-assembly of PS-PTCDI-PS in the polymer matrix. Of note is the difference between the OM and SEM images shown in Figures 7.1 (d), (e) and 7.2 (a) and (b). While the OM images show spherical domains, these are not seen in the SEM images. The 
surfaces in 7.2 (a) and (b) are smooth and do not indicate any phase separation. This difference was seen by Islam and Sundararajan ${ }^{8}$, in their studies on the self-assembly of phthalocyanine in polycarbonate and poly (methyl methacrylate) polymers. The self-assembly of phthalocyanine was so fast, that aggregation occurred even before the films dried. The molecules did not diffuse to the surface and crystallize as would normally happen during phase separation. As a consequence, these domains were in the bulk of film (sub-surface) and not on the surface of the film. Hence, we are able to see them in the transmission mode of the OM, but not in SEM. We observed that as-prepared films were transparent up to 5\% wt PS-PTCDI-PS after which crystallization occurs (Figure 7.3 (a) (b)). Profuse crystallization occurs in all concentrations of PS-PTCDI-PS in films prepared with THF (Figure 7.1 (f) and Figure 7.2 (c)). The behavior does not relate to the solubility parameter of polystyrene and the solvents.
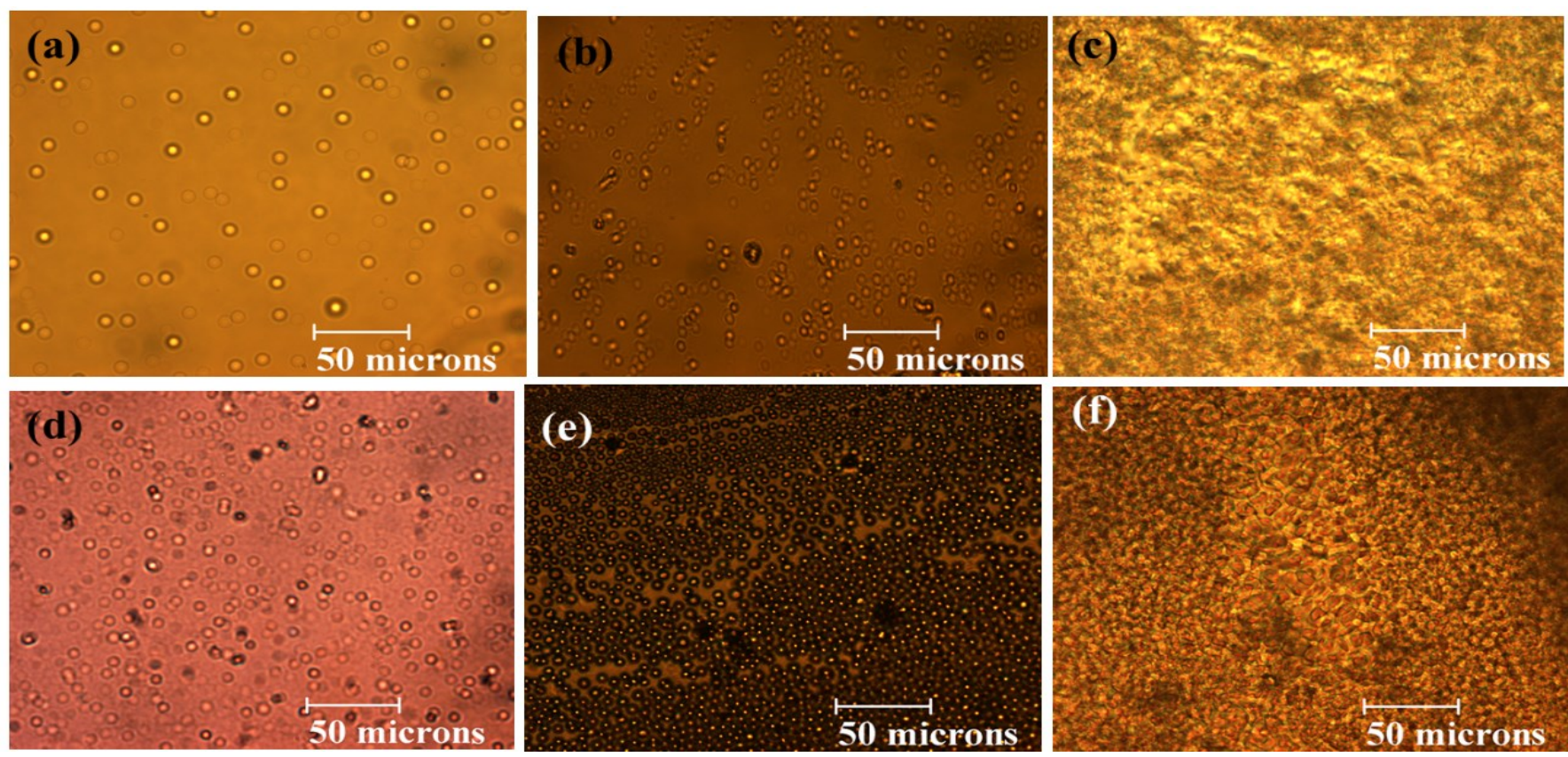

Figure 7.1 OM images of PS/PS-PTCDI-PS films cast from different solvents at different concentrations. $1 \mathrm{wt} \%$ PS-PTCDI-PS cast from (a) chlorobenzene (b) chloroform and (c) THF; $5 \mathrm{wt} \%$ PS-PTCDI-PS cast from (d) chlorobenzene (e) chloroform (f) THF 

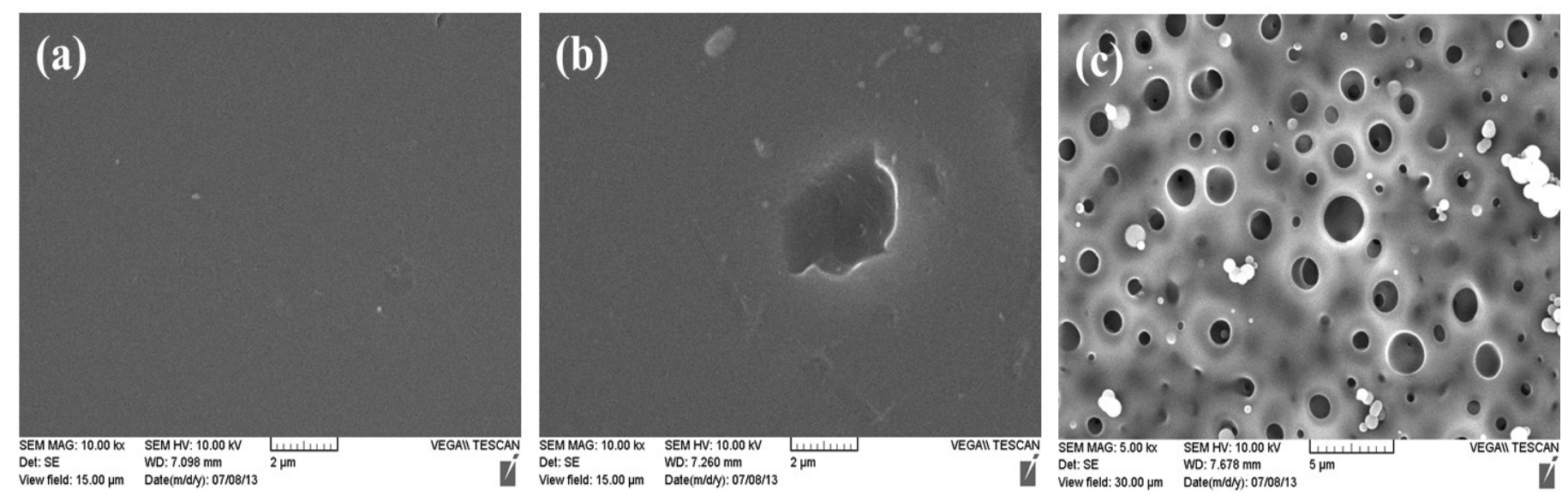

Figure 7.2 SEM images of films at $5 \mathrm{wt} \%$ PS-PTCDI-PS from (a) chlorobenzene (b) chloroform and (c) THF

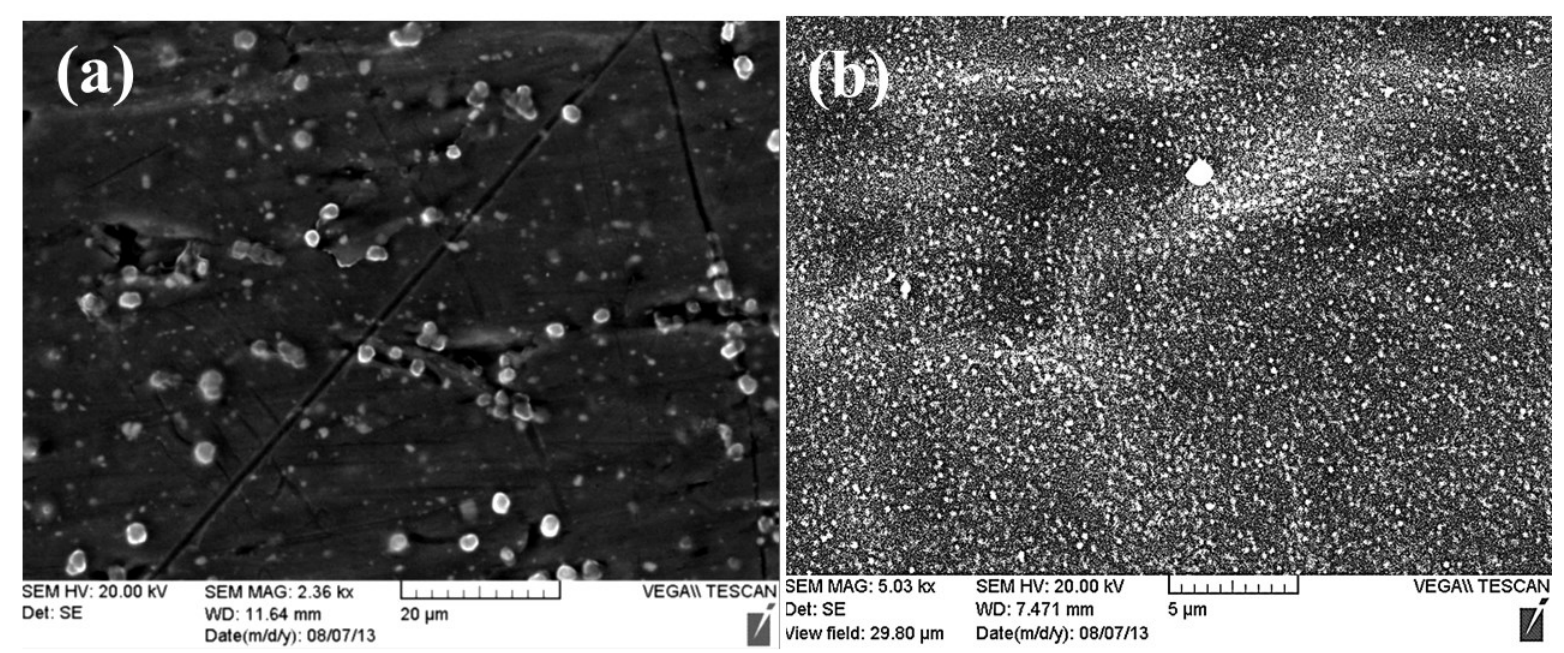

Figure 7.3 SEM of films at 10 wt \% PS-PTCDI-PS from (a) chlorobenzene (b) chloroform

Figure 7.4 (a), (b) shows that with $10 \mathrm{wt} \%$ PS-PTCDI-PS, the PS films lose their transparency. The choice of solvent also plays a role in determining the morphology of the films. In our previous studies ${ }^{13}$ on the self-assembly of PS-PTCDI-PS, no self-assembly was seen in solvents that are favourable for the polystyrene segment. Based on NMR data, it was proposed that the $\mathrm{C}-\mathrm{H} \ldots . . \pi$ interaction between the perylene and the aromatic protons of the phenyl side 
group of polystyrene prevented the self-assembly of the perylene. However, self-assembly was seen in films, upon evaporation of the solvent. A web morphology which arises from chaining of spherical particles was seen when chloroform was the choice of solvent. Stacking was seen in the films due to the collapsed conformation of the oligostyrene segment in these films upon evaporation of the solvent. In the present study, it is expected that a good solvent for the oligostyrene segment, which is also a non-solvent for the PTCDI is expected to promote the selfassembly of the latter in the polymer matrix. However, the morphology resulting from the selfassembly is different for films made with chloroform and chlorobenzene versus THF. 


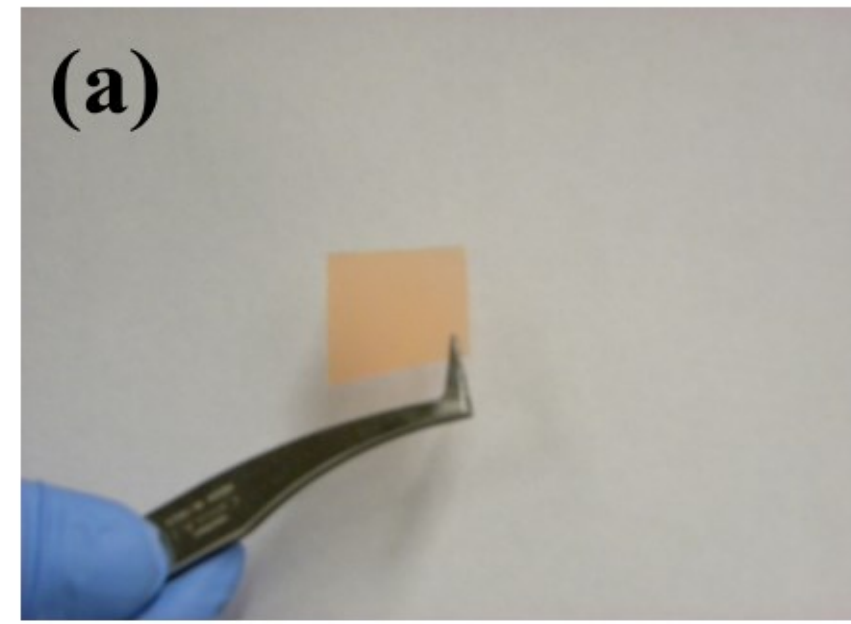

\section{(b)}

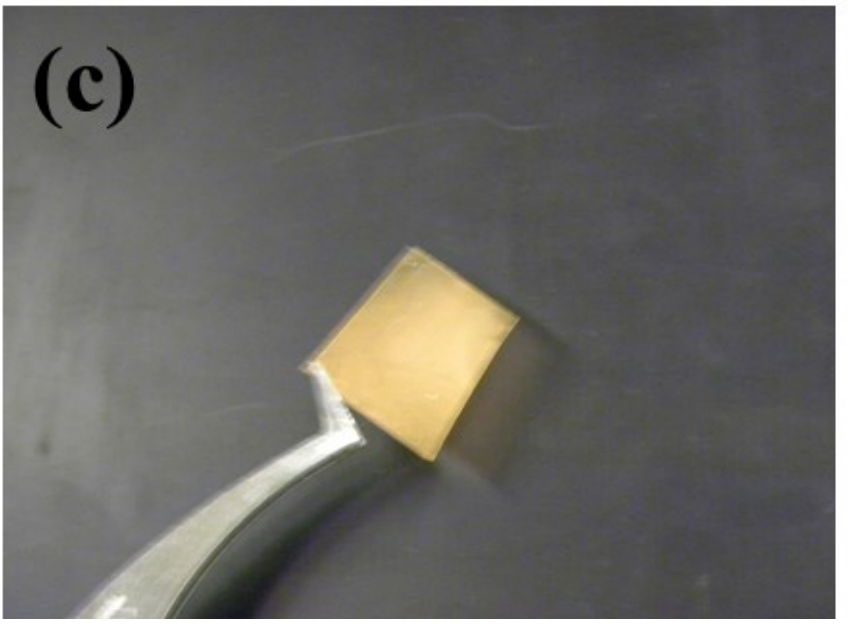

(d)
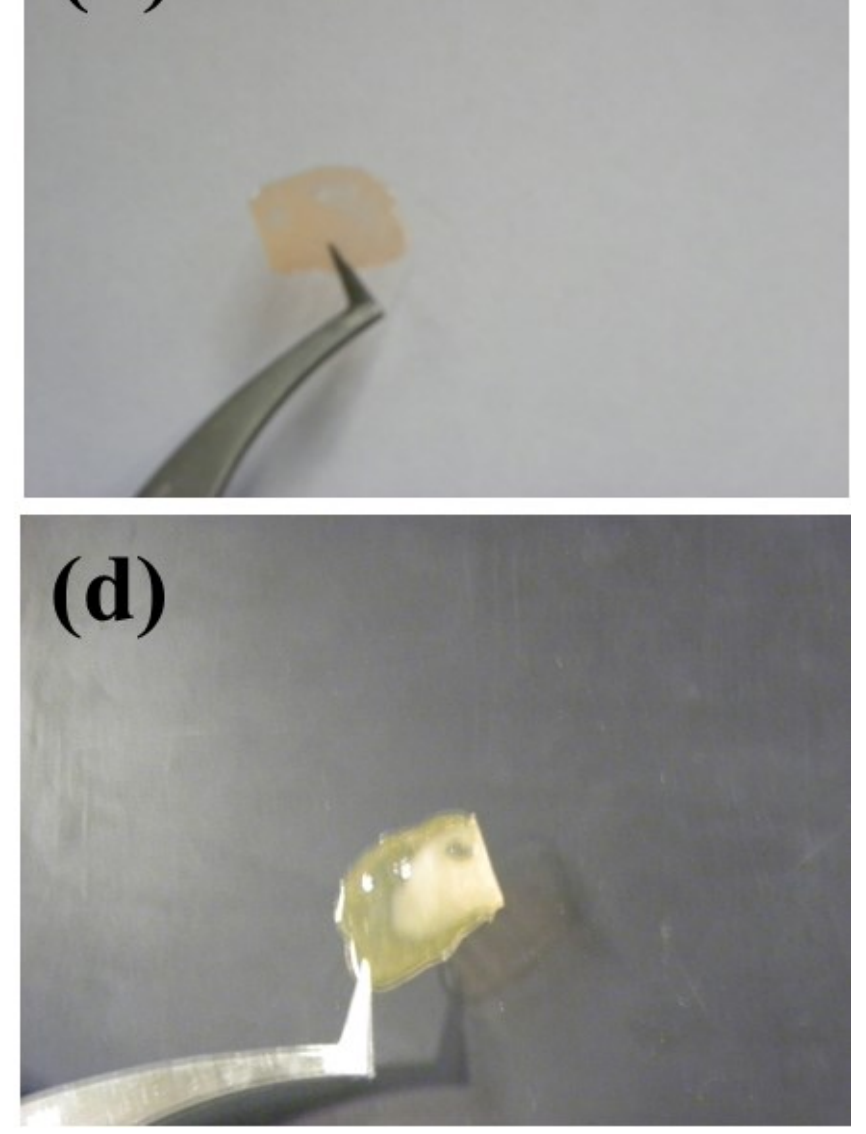

Figure 7.4 Physical appearances of the PS/PS-PTCDI-PS films. $5 \mathrm{wt} \%$ PS-PTCDI-PS cast from

(a) chlorobenzene (b) chloroform; $10 \mathrm{wt} \%$ PS-PTCDI-PS cast from (c) chlorobenzene 


\subsubsection{DSC Measurements}

Research on the relationship between the molecular weight of PS and glass transition has been widely reported in the past ${ }^{8-11}$. The $\mathrm{M}_{\mathrm{w}}$ of the PS used as the polymer matrix in this experiment is 239,700 . The $T_{g}$ of the films cast without the guest PS-PTCDI-PS, was found to be around $104^{\circ} \mathrm{C}$, which is expected for high $\mathrm{M}_{\mathrm{w}}$ PS. Figure 7.5 shows the variation of glass transition temperature as a function of PS-PTCDI-PS concentration in the PS polymer matrices when casting from the three different solvents. It can be seen that with the films cast from THF, which also showed a high degree of crystallinity and phase separation (section 7.1.1), the $\mathrm{T}_{\mathrm{g}}$ depression was the most obvious going from $104^{\circ} \mathrm{C}$ at $0 \mathrm{wt} \%$ PS-PTCDI-PS to $79{ }^{\circ} \mathrm{C}$ at $10 \mathrm{wt} \%$ PS-PTCDI-PS. With $5 \mathrm{wt} \%$ of PS-PTCDI-PS, the decrease in $\mathrm{T}_{\mathrm{g}}$ is just $4{ }^{\circ} \mathrm{C}$ when chloroform or chlorobenzene was used, while it is $14{ }^{\circ} \mathrm{C}$ when THF was used. The extent of depression is similar for all three solvents when the concentration of PS-PTCDI-PS increases to $10 \mathrm{wt} \%$. The differences in the $T_{\mathrm{g}}$ depression could be attributed to the molecular packing differences and the polymer conformation, which would in turn depend on the solvent used. The addition of the PSPTCDI-PS may cause an increase in the free volume of the polymer and this leads to enhanced mobility of the polymer and the depression of $\mathrm{T}_{\mathrm{g}}$. Moreover, the change in free volume of the polymer can occur either on the segment level or at the molecular level ${ }^{12}$. Thus the size of PSPTCDI-PS also affects the extent of $\mathrm{T}_{\mathrm{g}}$ depression. 


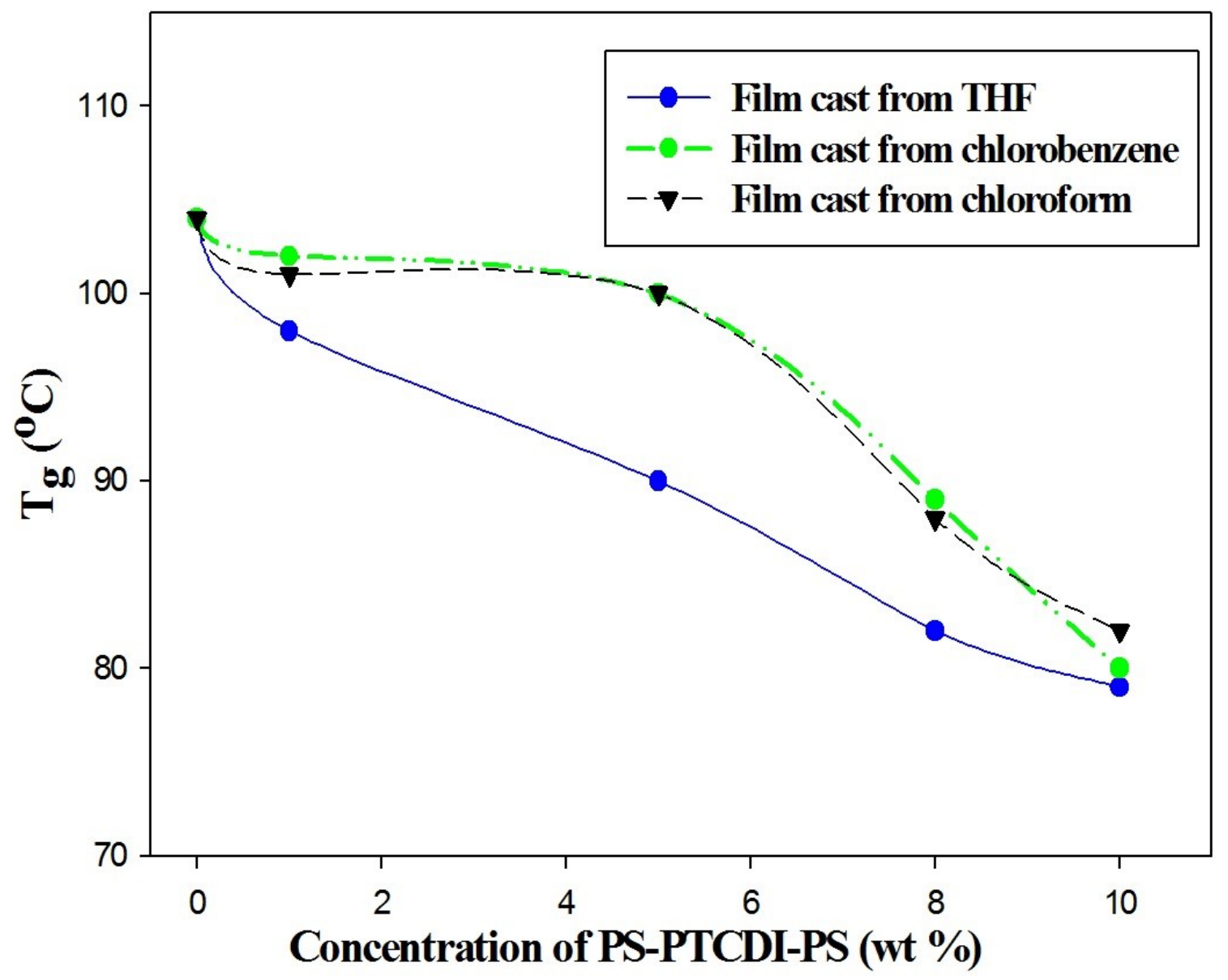

Figure 7.5 The variation of glass transition temperature is plotted as a function of PS-PTCDI-PS concentration in the PS polymer matrices using three different solvents 


\subsubsection{X-Ray Diffraction}

Figure 7.6 shows the XRD of the three types of films drop cast from different solvents. All the blends had a concentration of 5 wt \% PS-PTCDI-PS. The large hump (amorphous scattering) at about $2 \theta=20-25^{\circ}$ is due to the atactic polystyrene segment. With very few reflections, it is difficult to make any conclusions with regard to the packing of the molecules. However, the diffraction profiles of the films drop cast from chloroform and chlorobenzene have diffraction patterns that are similar; while the diffraction pattern of the films drop cast from THF shows extra peak. The extra peaks $8.04,6.41,4.08$ and 3.38 , and $3.06 \AA$ confirm the crystallinity of the films where THF was used, which may be due to aggregation of the perylene segment and phase separation of the guest PS-PTCDI-PS from the polymer matrix. 

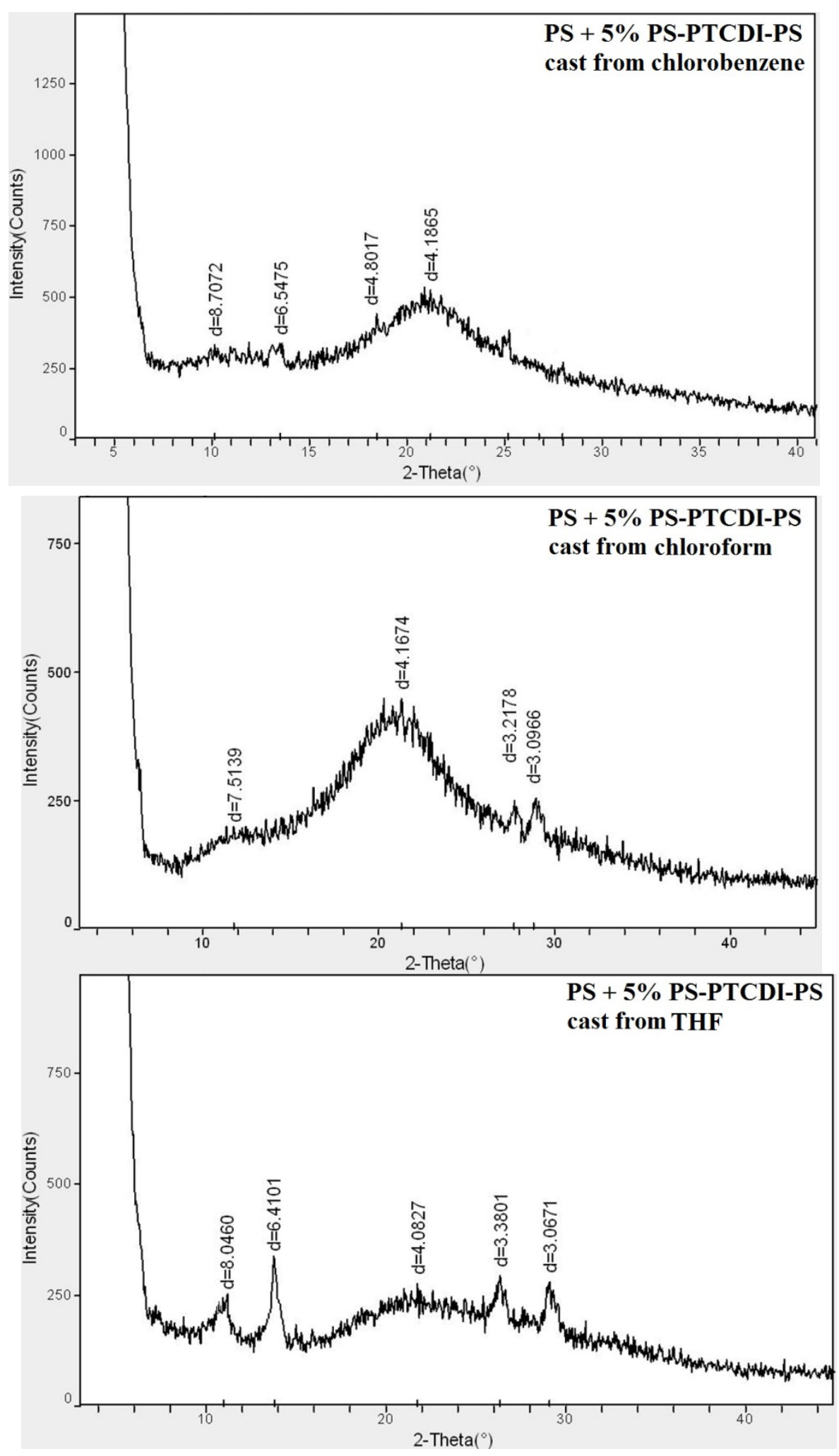

Figure 7.6 XRD patterns of three PS/ PS-PTCDI-PS (5 wt \%) films drop cast from different solvents 


\subsubsection{Optical Properties}

In our previous studies ${ }^{13}$, on the self-assembly of PS-PTCDI-PS, in various solvents, we found that the monomeric form of this compound in chloroform showed three distinct peaks in UV-Vis absorption studies. The peak positions originating from $\mathrm{S}_{0-2}, \mathrm{~S}_{0-1}$ and $\mathrm{S}_{0-0}$ vibronic transitions were at 456, 489 and $526 \mathrm{~nm}$ respectively. However as seen from figure 7.7 (b) even though PS-PTCDI-PS was confined in the PS polymer matrix, with the films, the self-assembly of PS-PTCDI-PS showed only two peaks that red shifted $498 \mathrm{~nm}$ and $532 \mathrm{~nm}$ relative to the monomeric form of PS-PTCDI-PS in solution. The films that were drop cast from chlorobenzene also showed a red shit to $494 \mathrm{~nm}$ and $528 \mathrm{~nm}$. However, when THF was used as the solvent, the UV-Vis spectrum showed the monomeric form of PS-PTCDI-PS. The film drop cast from THF showed a major peak at $526 \mathrm{~nm}$ corresponding to the $\mathrm{S}_{0-0}$ vibronic transition, a second peak at $491 \mathrm{~nm}$ corresponding to the $\mathrm{S}_{0-1}$ vibronic transition, and a hump at $461 \mathrm{~nm}$ corresponding to the $\mathrm{S}_{0-2}$ vibronic transition. It is clear from the microscopy data that phase separation of the PSPTCDI-PS from the polymer matrix occurs when THF is used as the solvent; however the PSPTCDI-PS is in its monomeric form. This is a discrepancy from our other data which suggest that the use of THF in casting the films, cause the crystallization and phase separation of PSPTCDI-PS to the surface. Hence it was expected that the UV-Vis spectra show the aggregate mode of PS-PTCDI-PS; further experiments will be needed to address these results. 

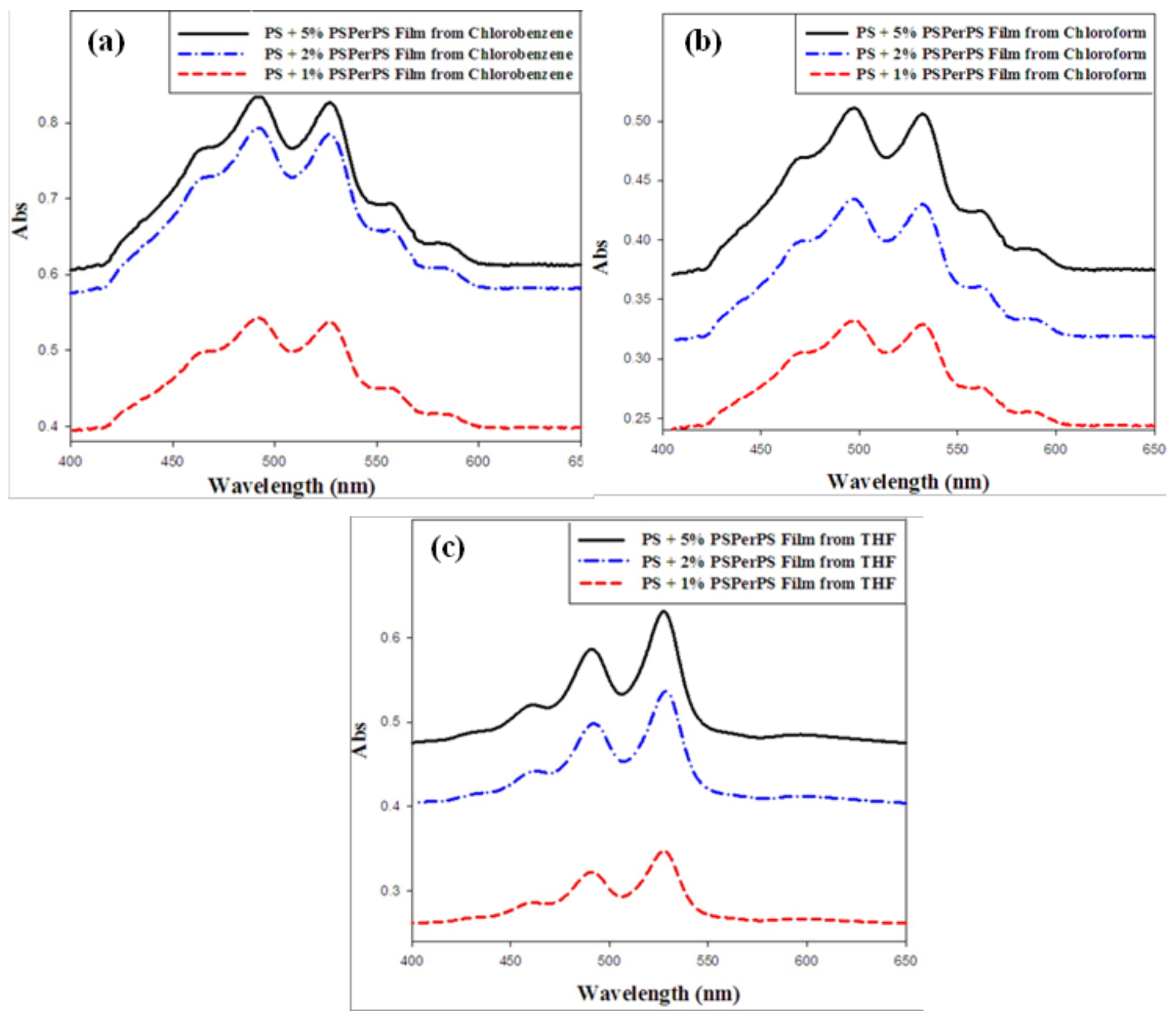

Figure 7.7 UV-Vis absorption spectra of films drop cast from different solvents at different PS-PTCDI-PS concentrations 


\subsubsection{IR Spectra}

When comparing the IR spectra of the pure PS films cast from chloroform versus the ones with 5 wt. \% PS-PTCDI-PS (Figure 7.8), it can be seen that there is a slight shift in the peak positions, as well as the increase in peak intensity. However even though the perylene segment is present, there are no extra peaks in the spectrum. When THF was used to drop cast the films (Figure 7.9), the spectrum changes. We see an extra peak at $1709 \mathrm{~cm}^{-1}$ corresponding to the asymmetric $\mathrm{C}=\mathrm{O}$ stretch of the perylene diimide and one at $1364 \mathrm{~cm}^{-1}$ which may be assigned to the symmetric $\mathrm{C}-\mathrm{N}$ stretch. The peak at $1026-1067 \mathrm{~cm}^{-1}$ may be assigned to the imide ring. Therefore it may be concluded from the IR spectra that when THF was used, phase separation occurs while if the other two solvents were used to drop cast, uniform films were obtained. 


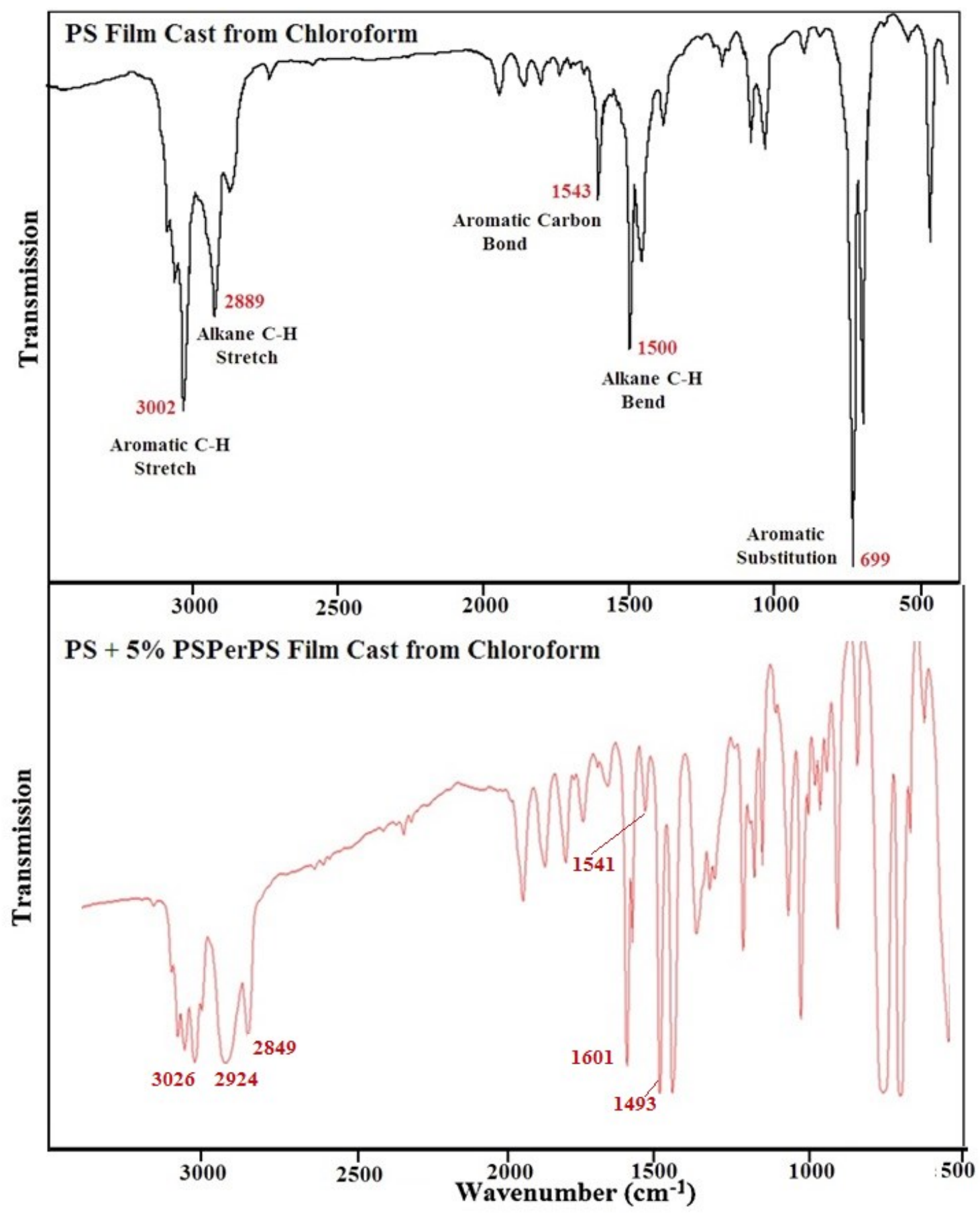

Figure 7.8 IR spectrum of pure PS film cast from chloroform (top) compared to that of the PS/PS-PTCDI-PS (5 wt \%) blended film cast from chloroform (bottom) 


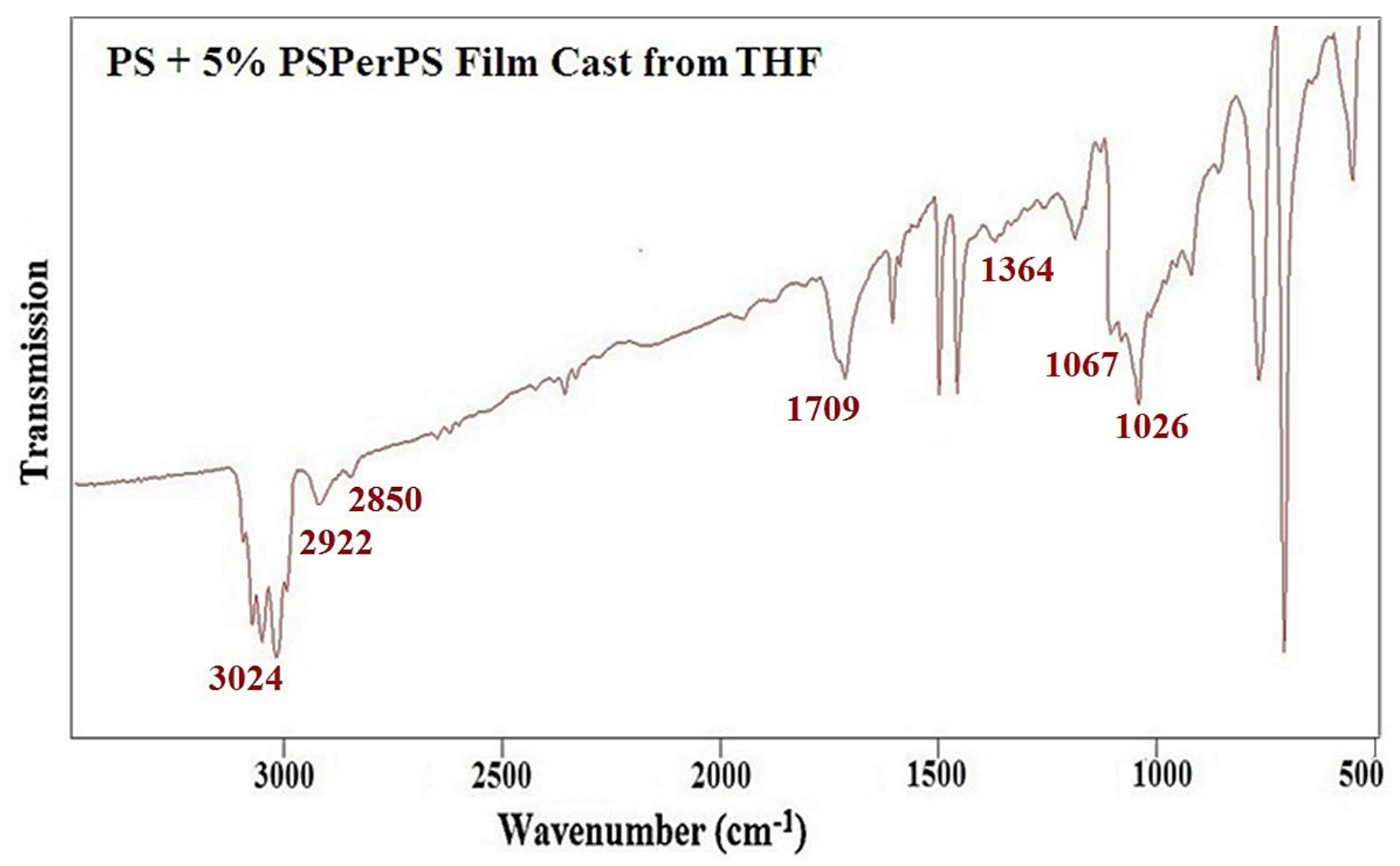

Figure 7.9 IR spectrum of PS/PS-PTCDI-PS ( $5 \mathrm{wt} \%$ ) blended film cast from THF 


\subsection{Conclusions and Future Directions}

In this chapter, we dispersed PS-PTCDI-PS in their corresponding PS polymer matrix. This was an attempt to make composite polymer films with the optical properties of the selfassembled PS-PTCDI-PS, we saw in chapter 6. These composite films had up to $5 \mathrm{wt} \%$ concentration of the host molecules which were shown to be compatible with the polymer matrix. This was proven by the transparency of the films and the retention of their physical properties i.e. no phase separation, while the self-assembly of PS-PTCDI-PS still occurred. Usually when a small molecule is dispersed in a polymer matrix, phase separation from the polymer matrix occurs; it would diffuse to the surface and form single crystals. This is not preferable since it has been shown by several studies that phase separation leads to defects in application purposes such as organo-electronic devices. While attaching oligostyrene to both sides of the PTCDI unit, was shown to increase compatibility between the guest PS-PTCDI-PS and the PS matrix, the choice of solvent is very important when drop casting the films. THF was not an ideal choice while chlorobenzene and chloroform were the solvents which caused the selfassembly of the guest molecules, through $\pi-\pi$ stacking of the perylene units, while properly dispersing the oligostyrene segments, leading to uniform films. 


\subsection{References}

1. Borsenberger, P. M.; Weiss, D. S. Organic Photoreceptors for Xerography, New York, Marcel Decker Inc., 1998.

2. Stolka, M.; Yanus, J. F.; Pai, D. M. Hole Transport in Solid Solutions of a Diamine in Polycarbonate. J. Phys. Chem. 1984, 88, 4707-4714.

3. Borsenberger, P. M. Hole Transport in Tri-Para-Tolylamine-Doped Bisphenol-APolycarbonate. J. Appl. Phys. 1990, 68, 6263-6273.

4. Lin, L. B.; Jenekhe, S. A.; Borsenberger, P. M. High Electron Mobility in Bipolar Composites of Organic Molecules. Appl. Phys. Lett. 1996, 69, 3495-3497.

5. Santerre, F.; Bedja, I.; Dodelet, J. P.; Sun, Y.; Lu, J.; Hay, A. S.; D’Iorio, M. Hole Transport Molecules in High $\mathrm{T}_{\mathrm{g}}$ Polymers: Their Effect on the Performance of Organic Light-Emitting Diodes. Chem. Mater. 2001, 13, 1739.

6. Khan, F.; Sundararajan, P.R. Influence of Polycarbonate Flexibility on the AnnealingInduced Phase Separation of the Hole Transport Molecule TPD in a Model Charge Transport Composite. J. Phys. Chem. B 2004, 108, 117-126.

7. Tuteja, B.; Moniruzzaman, M.; Sundararajan, P.R. Langmuir 2007, 23, 4709-4711.

8. Islam, M. R.; Sundararajan, P. R. Tubular or Subsurface Morphology of Octabutoxyphthalocyanine upon Self-Assembly in Polymer Matrices: Effect of the Casting Solvent. Chem. Eur. J. 2011, 17, 6098 - 6108.

9. Fox, T.G.; Flory, P.J. The Glass Temperature and Related Properties of Polystyrene Influence of Molecular Weight. J. Polym. Sci. 1954, 14, 315-319.

10. Schulz, G. V.; von Günner, K.; Gerrens, H. Z. Phys. Chem. N. F.1955, 4, 192. 
11. Couchman, P. R. The Effect of Degree of Polymerization on Glass-Transition Temperatures. Polym. Eng. Sci.1981, 21, 377.

12. Khan, F.; Khanna, S.; Hor, A.; Sundararajan. P.R. The Role of Molecular Volume and the Shape of the Hole Transport Molecule in the Morphology of Model Charge Transport Composites. Can. J. Chem. 2010, 88, 247-259.

13. Islam, ,M.; Sundararajan, P.R. Nano-Scale Self-Assembly Impeded by $\mathrm{CH} \cdots \pi$ Interaction in Block Selective Solvents in the Case of Oligostyrene-Perylene diimide-Oligostyrene (Coil-Rod-Coil) Molecule. Eur. Polym. J. 2013, 49, 2042-2051. 


\section{Conclusions and Future Directions}

Achieving supramolecular control over chromophore-linked molecular systems continues to be a challenge. The main challenge of 1D self-assembly of PDI molecules lies in controlling and optimizing the strong $\pi-\pi$ interaction between the perylene planes and the intermolecular interactions between the side chains. The self-assembly of the gelator depends upon a variety of factors including the solvent, concentration, temperature, and, above all, the molecular structure. A minimal structural alteration such as the length of the flexible side chain of a gelator is often crucial for the manipulation of the gelation-induced properties depending on the subtle hydrophobic/ hydrophilic balance. For example, most gelators have been reported to form a gel in a single solvent. However, a mixture of solvents could enhance the supramolecular organization of the gelator. Therefore, an optimum balance between the hydrophilic and hydrophobic groups in the gelator, obtained either by varying the molecular structure or changing the polarity of a solvent mixture, could control the gelation-assisted properties.

The preparation of gels of poly(dimethylsiloxane) (PDMS) reported in the literature so far involves catalysts and chemical cross-links (chemical gels) or functionalization with organogelators. In this thesis we reported that thermo-reversible physical gels of PDMS, without cross-links or functionalization, can be made with propylamine or hexylamine as a solvent. The gels consist of spherical domains as small as $20 \mathrm{~nm}$. We showed that these spherical domains are

part of a network. Differential scanning calorimetry (DSC), optical microscopy, and rheology show that the gel is thermo-reversible. With the DSC experiments, we have devised a procedure to achieve thermo-reversibility with very similar gel-sol transition endotherms in the first and second heating cycles. 
Organogels with aromatic molecules such as perylene derivatives and phthalocyanines reported in the literature so far involve self-assembly promoted by hydrogen bonds, in addition to aromatic and van der Waals interactions. In this thesis we presented a case of thermoreversible gelation without a hydrogen bonding group in the structure of (a) a coil-rod-coil molecule based on perylenetetracarboxylic diimide (PTCDI) and poly (dimethyl siloxane) (PDMS) and (b) a rod-coil molecule with perylene dicarboxylic imide (PDI) and PDMS. In addition, publications so far on gelation of perylene diimide based molecules involve groups attached to both imide nitrogens and with or without substitution in the bay position. We discussed here the gelation with a mono-substituted perylene imide. The PDMS segment was attached to one side of PDI (Mono-PDMS) or to both imide nitrogens of PTCDI (Di-PDMS). The Mono-PDMS is an inverse macromolecular surfactant applicable to non-aqueous systems, and the Di-PDMS is a Gemini surfactant. The PDMS segment that we attached to PTCDI here is longer than most substituents used by other authors. These molecules gel propylamine, as well as mixed solvents of hexane/water and diisopropylamine/water. Both hexane and diisopropylamine dissolve Mono-PDMS and Di-PDMS at room temperature and addition water results in precipitation. However, heating the solution to about $70{ }^{\circ} \mathrm{C}$, adding water $(5-15 \% \mathrm{wt})$ and slowly cooling the solution, lead to gelation. The Di-PDMS forms fibers which are not flat but curved as an eaves trough. The Mono-PDMS forms hollow spheres.

Our next step was to attach a water-soluble side chain to PDI. Here we described the gelation caused by PDI, substituted with Jeffamine ${ }^{\circledR}$ on one imide nitrogen (MJ-PTCDI) or both (DJ-PTCDI). We used Jeffamine M-2070 polyetheramine, which is a propylene oxide/ethylene oxide copolymer (PO/EO mole ratio of 10/31). In this case again, there is no hydrogen bonding group $(\mathrm{N}-\mathrm{H}, \mathrm{C}=\mathrm{O})$ in the side chain structure. As with the Di-PDMS and Mono-PDMS, the DJ- 
PTCDI is a Gemini surfactant and MJ-PTCDI is an inverse macromolecular surfactant. Formation of worm-like micelles was reported recently, during the association of Gemini surfactants. The morphology of the gels reported here, depends on the amount of solvent: nonsolvent ratio. DJ-PTCDI formed spheres that were secondarily aggregated to long fibers. MJPTCDI also forms vesicles that are fused together, forming honeycomb morphology. These are also examples of worm-like vesicles.

For our final gelator, we attached oligostyrene to both sides of the PTCDI (PS-PTCDIPS), to form a coil-rod-coil molecule, and studied its gelation behavior. This differs from the case of coil-rod-coil molecules previously discussed. With Di-PDMS, the $T_{g}$ of the PDMS segment is $-125^{\circ} \mathrm{C}$. With DJ-PTCDI, considering that the glass transition temperatures of PEO and PPO are -50 and $-75^{\circ} \mathrm{C}$, respectively, the $\mathrm{T}_{\mathrm{g}}$ of Jeffamine can be expected to be about $-60^{\circ} \mathrm{C}$. However, the oligostyrene segment that we used here has a $\mathrm{T}_{\mathrm{g}}$ of $43{ }^{\circ} \mathrm{C}$. Further, while the rod segment is a large aromatic chromophore, and the oligostyrene coil has aromatic side groups. Since a-PS has a planar $\pi$-bonding side group, we investigated whether additional support to the $\pi-\pi$ interaction of the perylene units would enhance aggregation and hence gelation.

Usually when a small molecule is dispersed in a polymer matrix, phase separation from the polymer matrix occurs; it would diffuse to the surface and form single crystals. This is not preferable since it has been shown by several studies that phase separation leads to defects in application purposes such as organo-electronic devices. In this thesis we use PS-PTCDI-PS as the guest and PS as the polymer matrix, While attaching oligostyrene to both sides of the PTCDI unit, was shown to increase compatibility between the guest PS-PTCDI-PS and the PS matrix, the choice of solvent was very important when drop casting the films. THF was not an ideal choice while chlorobenzene and chloroform were the solvents which caused the self-assembly of 
the guest molecules, through $\pi-\pi$ stacking of the perylene units, while properly dispersing the oligostyrene segments, leading to uniform films. Further research on these composite polymer films or gels will shed light on understanding their optical properties due to the presence of the perylene units. We propose that this research be done on the other coil-rod and coil-rod-coil polymers used in the other chapters, with their corresponding polymers in order to investigate the mechanical and barrier properties of these unique polymer-polymer composite interactions among the compatible polymers used. For example, the study of Di-PDMS and Mono-PDMS dispersed in PDMS films as optically responsive PDMS films or PDMS gels (physical or cross linked). Similarly, DJ-PTCDI and MJ-PTCDI loaded in PEO may be studied as sensors for potential applications in cell research. 
APPENDIX 
Appendix 1 From: Yao, D.; Bender, T. P.; Gerroir, P. J.; Sundararajan, P. R. Macromolecules 2005, 38, 6972

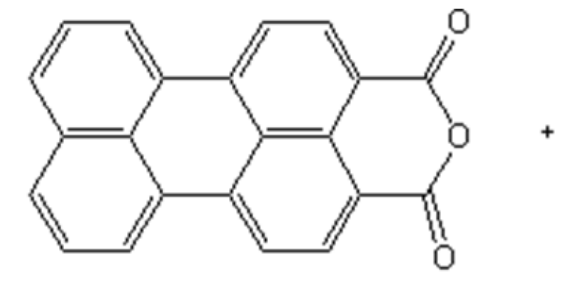

4

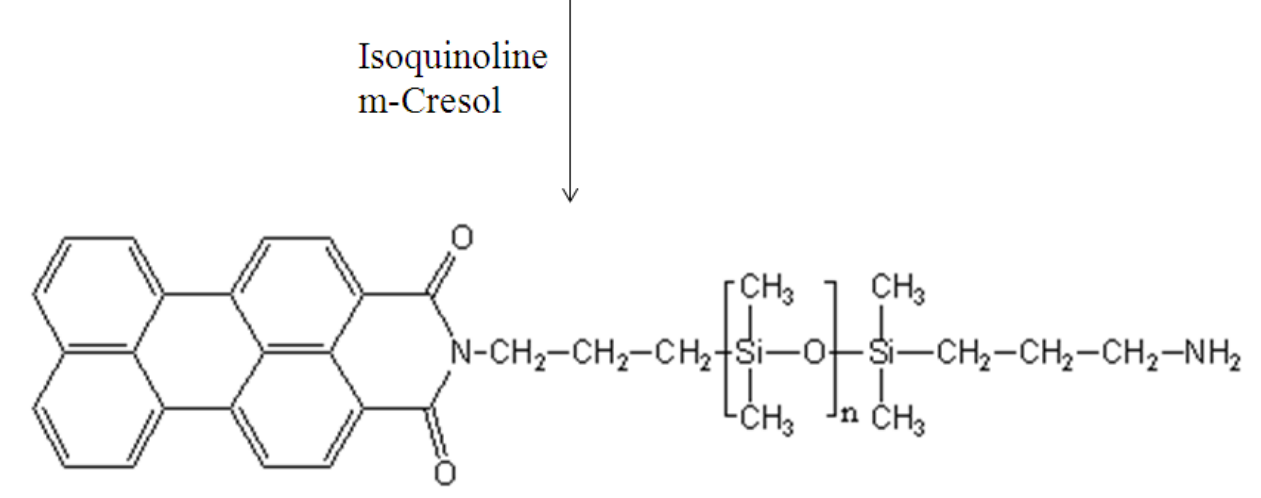

$6,7,8$

Scheme A 1.1 Synthesis of PDMS-end-perylene: (6) $n=10 ;(7) n=20 ;(8) n=40$
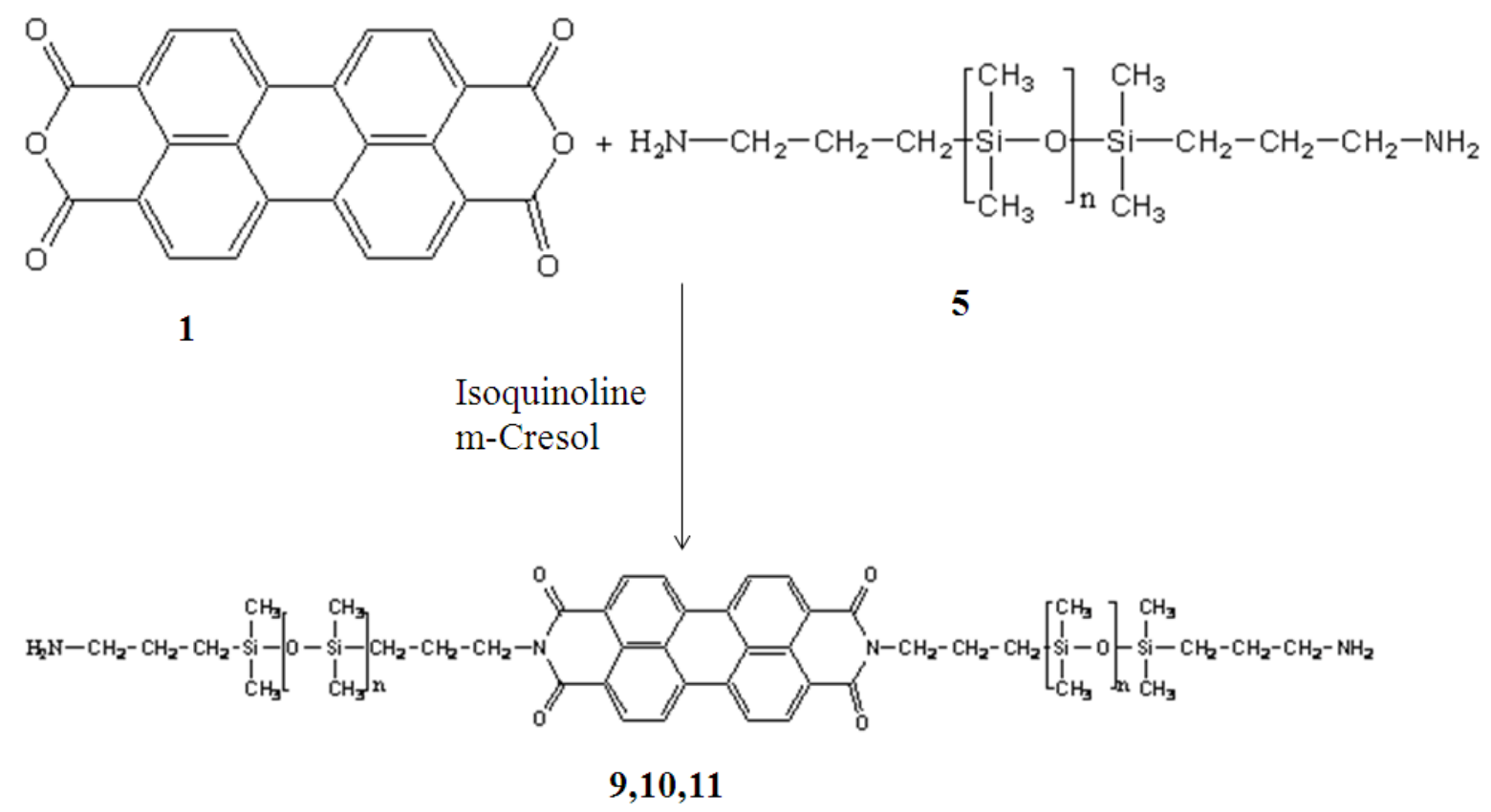

Scheme A 1.2 Synthesis of PDMS perylene bisimides: (9) n=10; (10) n=20; (11) n=40 
Mono-PDMS was prepared by Yao et al. by reaction of perylene-3,4-dicarboxylic anhydride with an amino terminated polysiloxane in m-cresol at elevated temperatures (compounds 6,7 and 8, Scheme A 1.1 ). A 4-fold excess of the amino terminated polysiloxane was used so as to preferentially synthesize polysiloxane that are endcapped at only one end with perylene moieties. The crude materials were washed by $1 \mathrm{~N}$ aqueous sodium hydroxide, in order to change any unreacted perylene 3,4-dicarboxylic anhydride to disodium perylene dicarboxylate and remove it. The column chromatography can remove trace amino-terminated PDMS and other unpurified materials. The reaction was monitored using FT-IR, by observing the wave number of carbonyl group shift from a broad peak between 1730 and $1780 \mathrm{~cm}^{-1}$ for the anhydride to peaks at 1690 to $1650 \mathrm{~cm}^{-1}$ upon imide formation. ${ }^{1} \mathrm{H}$ NMR $\left(\mathrm{CDCl}_{3}\right.$ solution) further confirmed the formation of perylene end-cap PDMS as chemical shifts between 8.0-8.6 ppm correspond to perylene hydrogens, 0.7-4.2 $\mathrm{ppm}$, due to the hydrogens of the alkyl groups $(\alpha-, \beta$ - and $\gamma$ - to the nitrogen atom) and chemical shifts lower than 0.5 due to hydrogens of the methyl groups attached directly to the silicon atoms were observed (Figure A 1.1).

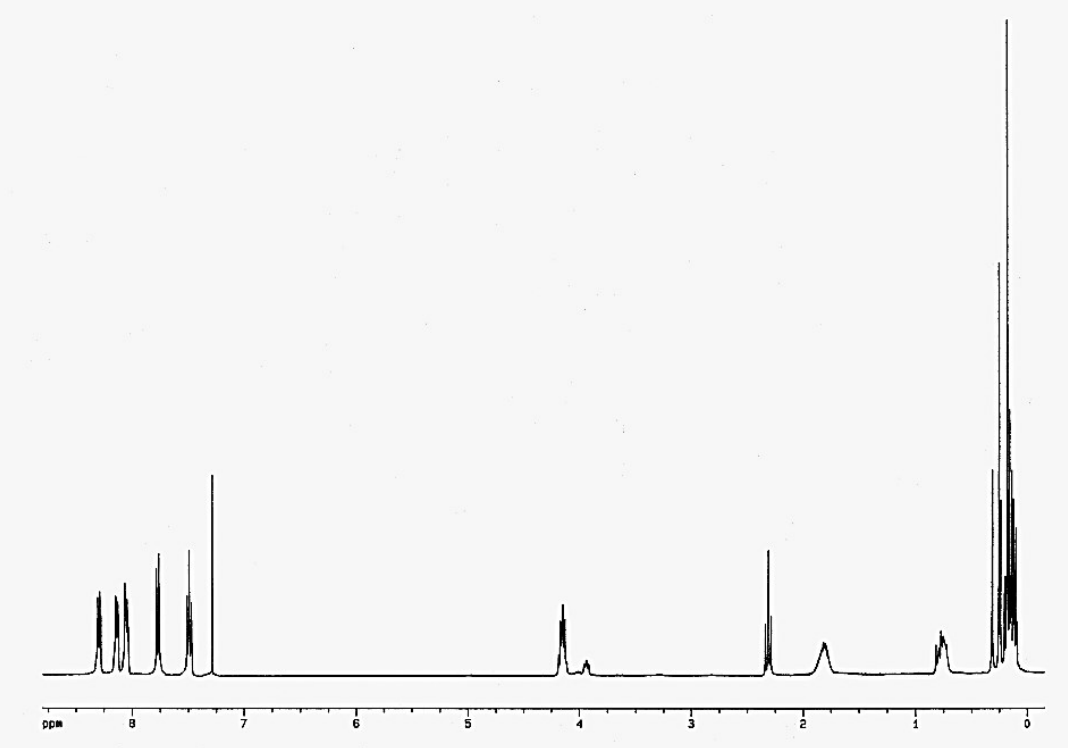

Figure A 1.1 ${ }^{1} \mathrm{H}$ NMR of Mono-PDMS using 400MHz NMR instrument. 
For comparison with PDMS-end-perylene, Yao et. al. also synthesized Di-PDMS by reaction of perylene 3,4,9,10-tetracarboxylic dianhydride with amino terminated polysiloxane in m-cresol at elevated temperatures. The reaction condition is same as (PDMS-end-perylene), except that perylene 3,4,9,10-tetracarboxylic dianhydride should be slowly added into the solution of amino terminated polysiloxane, isoquinoline, and $\mathrm{m}$-cresol, so that only 2 moles of amino terminated poly(dimethyl siloxane) react with 1 mole of perylene 3,4,9,10-tetracarboxylic dianhydride. The appearances of PDMS perylene bisimides are similar to PDMS-end-perylene. All of them are dark red. However, the solubility is lower than PDMS-end-perylene. ${ }^{1} \mathrm{H}$ NMR $\left(\mathrm{CDCl}_{3}\right.$ solution $)$ shows that 0.7-4.2 ppm, due to the hydrogens of the alkyl groups $(\alpha-, \beta$ - and $\gamma$ - to the nitrogen atom), between $8.4-8.6 \mathrm{ppm}$ correspond to perylene hydrogens, and chemical shifts lower than 0.5 due to hydrogens of the methyl groups attached directly to the silicon atoms were observed (Figure A 1.2)

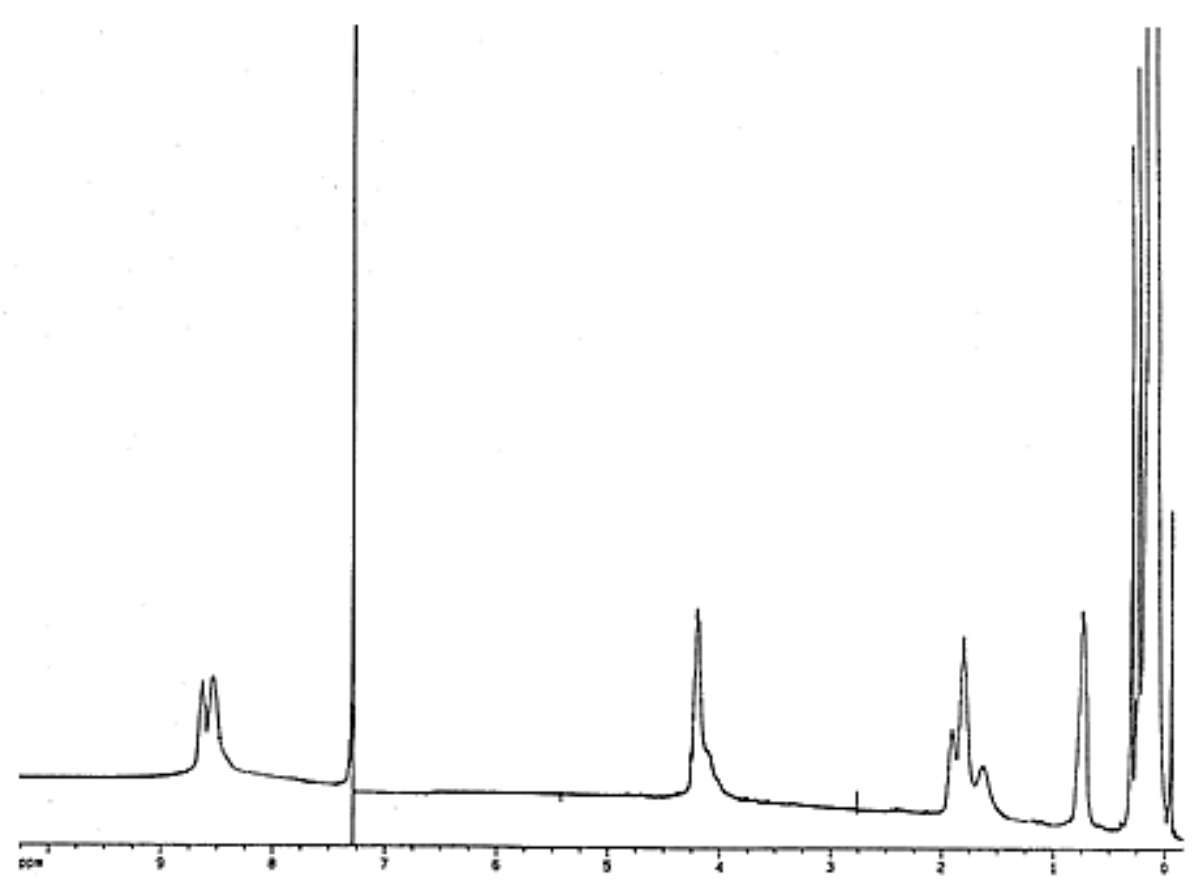

Figure A 1.2 1H NMR of Di-PDMS 
Appendix 2 From: Islam, R. M.; Dahan, E.; Saimani, S.; Sundararajan, P.R. Eur. Polym. J. 2012, 48, 1538-1554

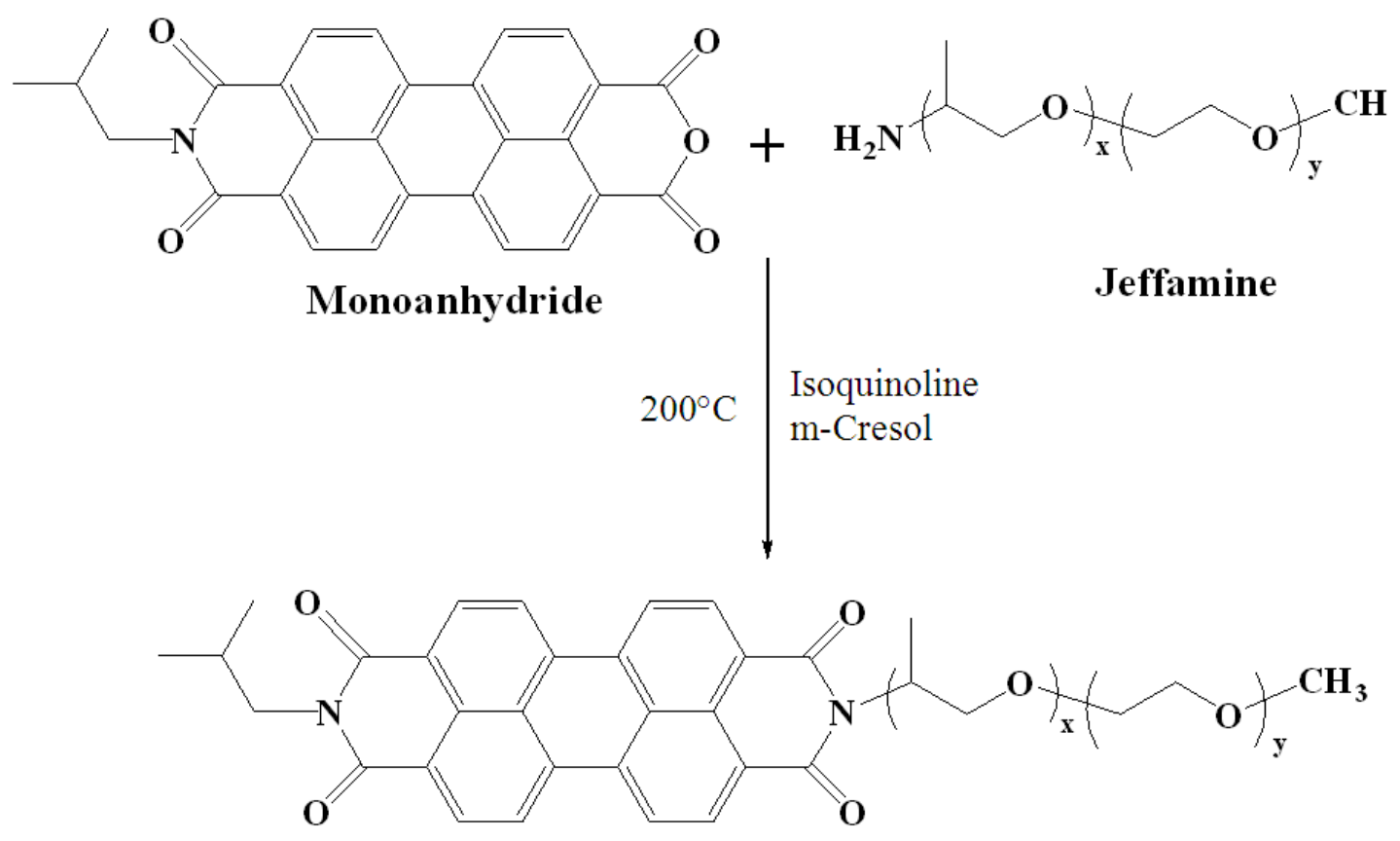

Scheme A2.1 Synthesis of MJ-PTCDI

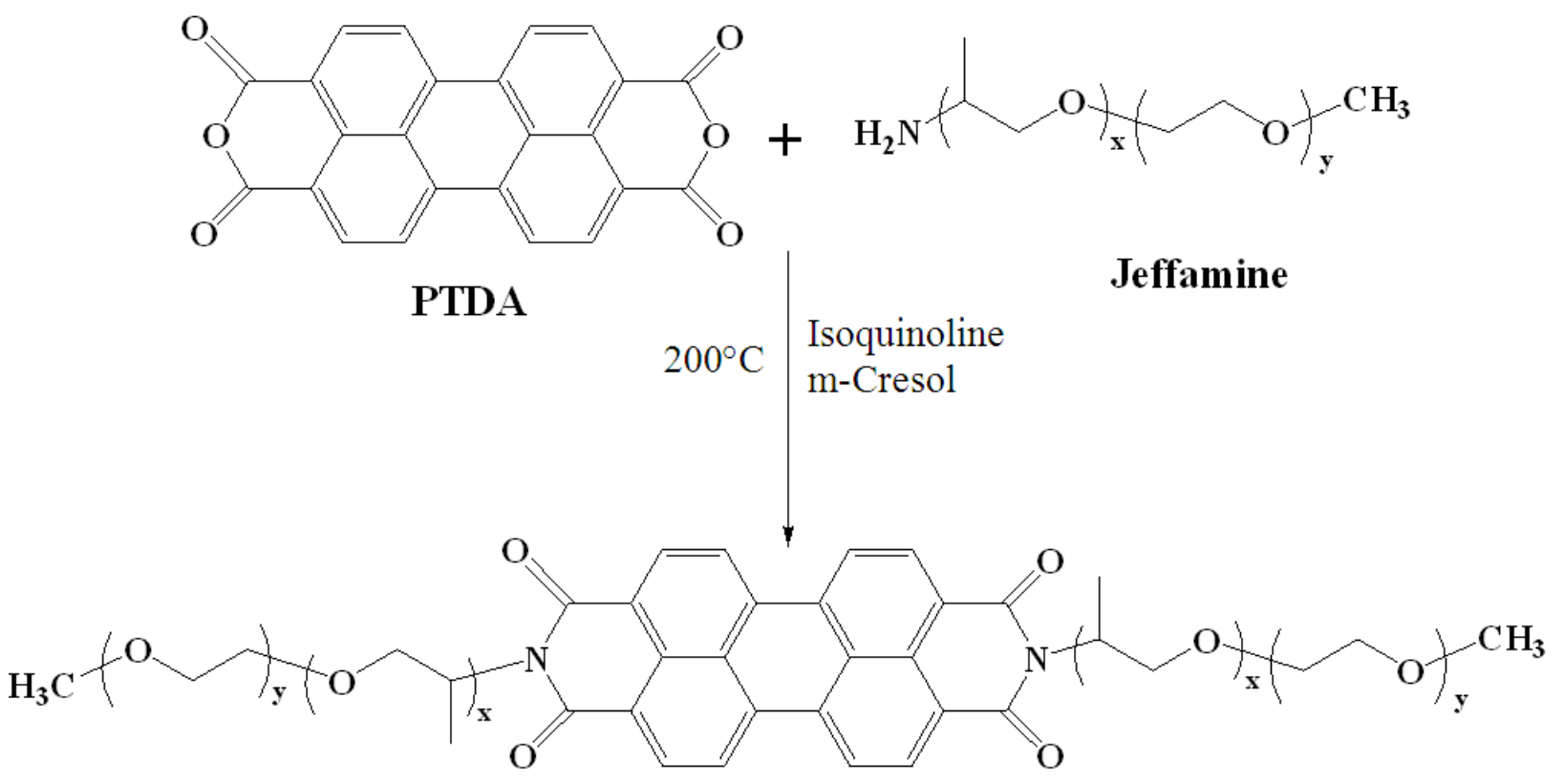

Scheme A 2.2 Synthesis of DJ-PTCDI 
MJ-PTCDI was prepared by Islam et al. by reacting PTDA and mono amino-terminated Poly(ethylene oxide-co-propyleneoxide) monomer (Jeffamine) in the usual procedure. Both the monomers are dispersed in $\mathrm{m}-\mathrm{Cresol}$ and imidized at $200^{\circ} \mathrm{C}$ and the completion of imidization was confirmed by FTIR. The FTIR spectra of the MJ-PTCDI is given in figure A 2.1. The $>\mathrm{C}=\mathrm{O}$ vibration of the anhydride (1773 and1757 $\left.\mathrm{cm}^{-1}\right)$ shifted to lower frequency upon conversion into imides. The symmetric and asymmetric absorption of the imide carbonyl group was observed around 1698 and $1656 \mathrm{~cm}^{-1}$, respectively. The complete disappearance of anhydride carbonyl vibrations indicates the absence of starting materials. The out-of-phase bending of the imide ring was observed around $747 \mathrm{~cm}^{-1}$. The C-N-C stretching vibration of the imide ring was observed around $1372 \mathrm{~cm}^{-1}$.

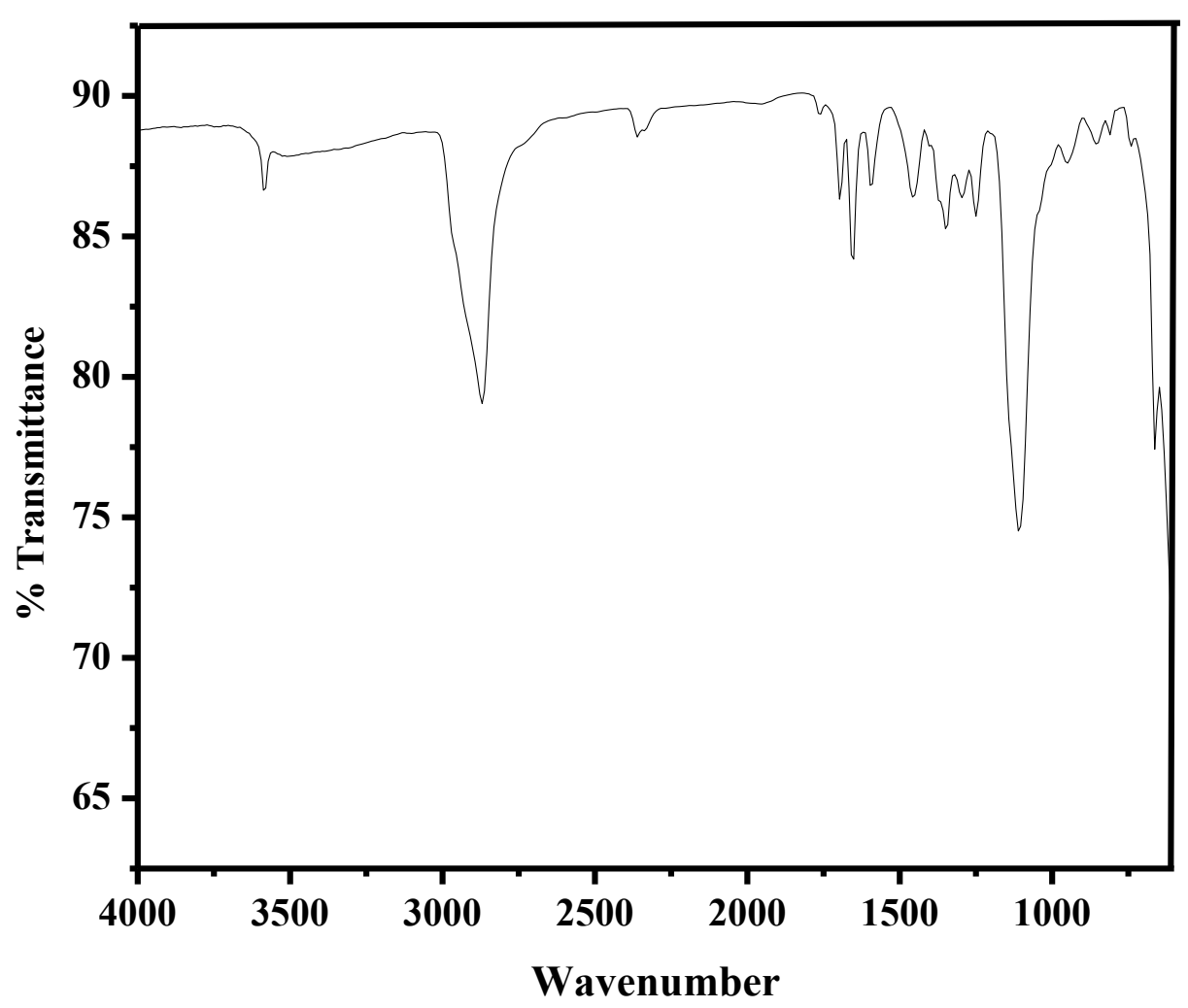

Figure A 2.1 FT-IR Spectrum of MJ-PTCDI 
DJ-PTCDI was also synthesized by Islam et. al. In a round-bottom flask, $0.1 \mathrm{mmol}$ of 3,4,9,10-perylenetetracarboxylic dianhydride, $0.2 \mathrm{mmol}$ of Jeffamine ${ }^{\circledR}$, and $0.2 \mathrm{~g}$ of isoquinoline were dispersed in $15 \mathrm{~mL}$ of $\mathrm{m}$-cresol, and the solution was slowly heated to $200^{\circ} \mathrm{C}$. The reaction mixture was stirred at $200{ }^{\circ} \mathrm{C}$ for $4 \mathrm{~h}$, cooled to room temperature and poured into chloroform. The solution was filtered to remove the unreacted 3,4,9,10-perylenetetracarboxylic dianhydride if any. The filtrate was collected and it was washed with acidified water in a separating funnel to remove the unreacted Jeffamine, if any and the organic layer was separated and dried to yield a red waxy material (yield 85\%). FTIR (KBr, Chloroform): 2858 (-C-H stretching), 1698 (imide), 1656 (imide), 1594 (C-C stretching), 1457(C-H bending), 1094 (C=O stretching), 850.1(Ar-CH out of plane bending), 747.3 (C-H rocking) $\mathrm{cm}^{-1}$. In the FT-NMR spectra, the resonance from the perylene moiety in the aromatic region (8.8-8.6 ppm, multiplet) and the ethylene oxide moiety in aliphatic region also confirmed the completion of reaction (Figure A 2.2)

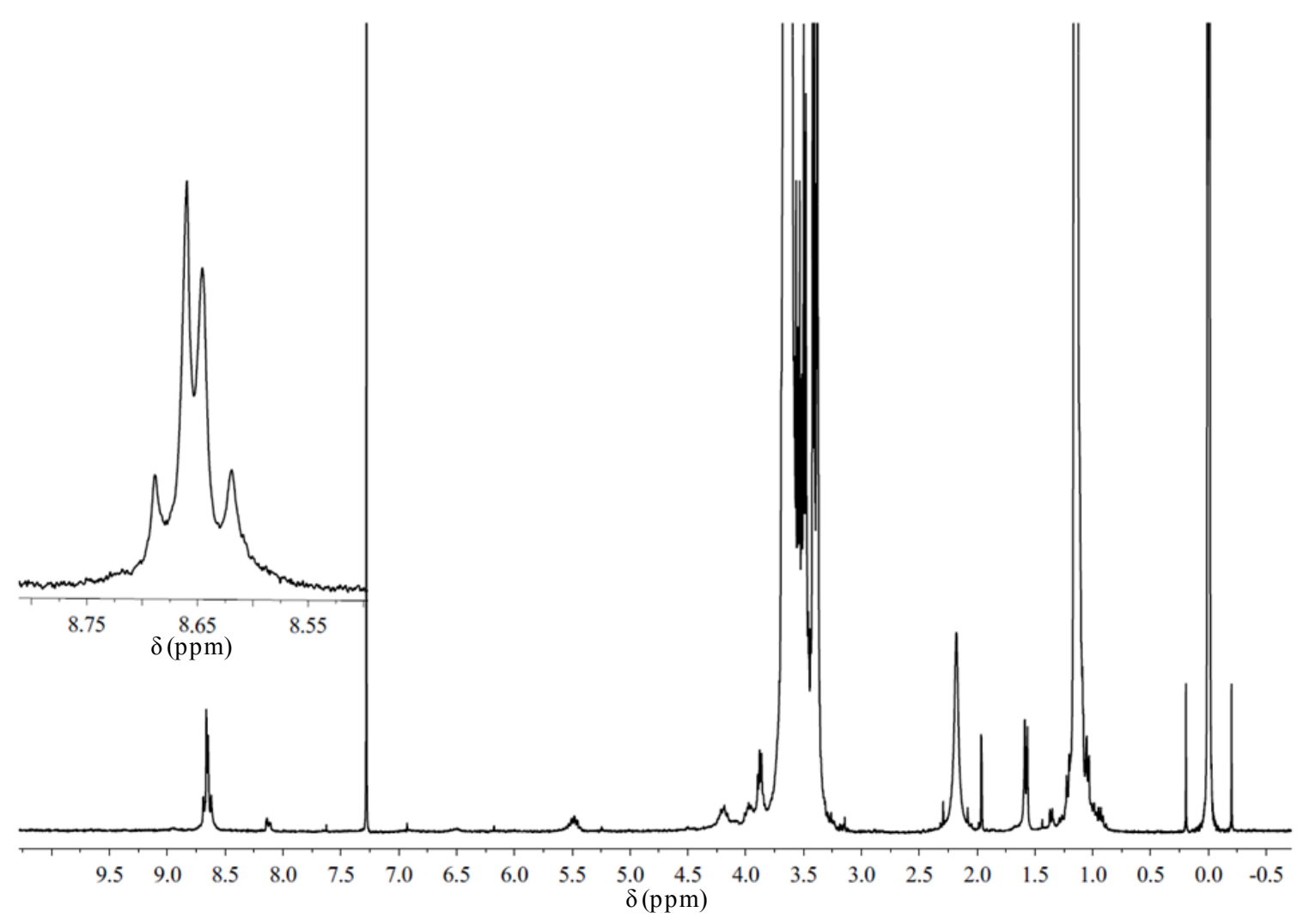

Figure A 2.2 ${ }^{1} \mathrm{HNMR}$ spectra of DJ-PTCDI in CDCl3. 
Appendix 3 From: Islam, M..; Sundararajan, P.R. Eur. Polym. J. 2013, 49(8), 2042-2051.
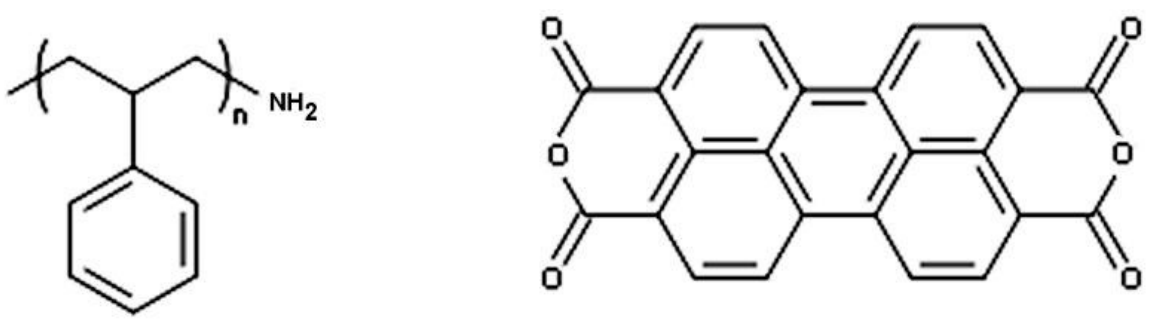

1) DMAc, $\mathrm{Zn}(\mathrm{Oac})_{2}, 2 \mathrm{H}_{2} \mathrm{O}, 110^{\circ} \mathrm{C}, 1 \mathrm{hr}$

2) $\mathrm{PTDA}, 110^{\circ} \mathrm{C}, 3 \mathrm{hrs}$

3) $60^{\circ} \mathrm{C}, 16 \mathrm{hrs}$

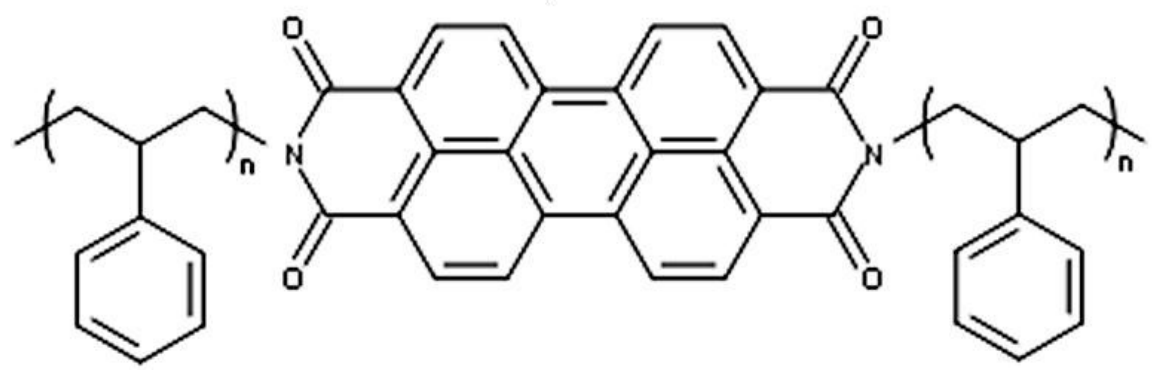

Scheme A 3.1 Synthesis of PS-PTCDI-PS

PS-PTCDI-PS was prepared by Islam et. al. In a round-bottom flask amino terminated polystyrene $(0.40 \mathrm{~g}, 0.33 \mathrm{mmol})$, zinc acetate $(0.001 \mathrm{~g}, 0.006 \mathrm{mmol})$ in N,N-dimethylacetamide $(25$ $\mathrm{mL})$ were heated in an oil bath attached with a condenser for 1 hour with stirring at $110{ }^{\circ} \mathrm{C} .3,4,9,10$ perylenetetracarboxylic dianhydride $(0.03 \mathrm{~g}, 0.08 \mathrm{mmol})$ was added, and the reaction mixture was stirred vigorously at $110{ }^{\circ} \mathrm{C}$ for $3 \mathrm{~h}$ and then continued at $160{ }^{\circ} \mathrm{C}$ for another $16 \mathrm{~h}$ keeping the same set up. The excess solvent was distilled off, and the slurry was washed several times with benzene to remove unreacted amino terminated polystyrene and dissolved in chloroform. The solution was filtered to remove unreacted 3, 4, 9, 10-perylenetetracarboxylic dianhydride if any. The chloroform solution was extracted with acidic distilled water three times and then with distilled water a few more times until the aqueous phase $\mathrm{pH}$ is neutral. The extracted chloroform solution was dried over 
sodium sulphate. The solution was then evaporated to get the final product, which was kept in a vacuum chamber for $24 \mathrm{hrs}$ to remove any solvent and dried to get a red product (yield $78 \%$ ). The FTIR spectra revealed the presence of imide carbonyl absorption frequencies $\left(1697\right.$ and $\left.1655 \mathrm{~cm}^{-1}\right)$ confirming the formation of the compound (Figure A 3.1) as a diimide of PS. ${ }^{1} \mathrm{HNMR}$ spectra also confirmed the presence of perylene group giving a multiplet at around $8.6 \mathrm{ppm}$ (Figure A 3.2)

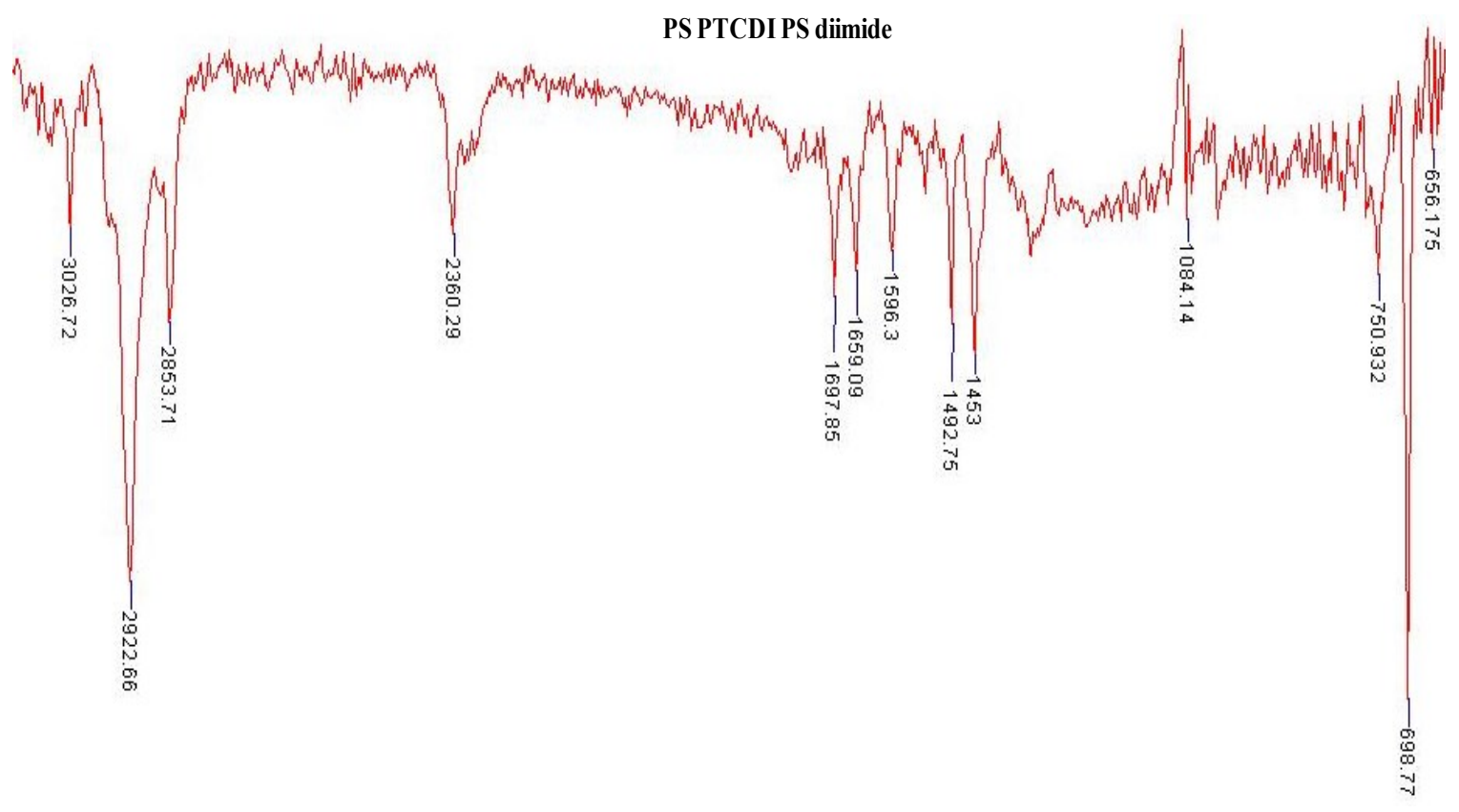

Figure A 3.1 FTIR spectra of PS-PTCDI-PS 


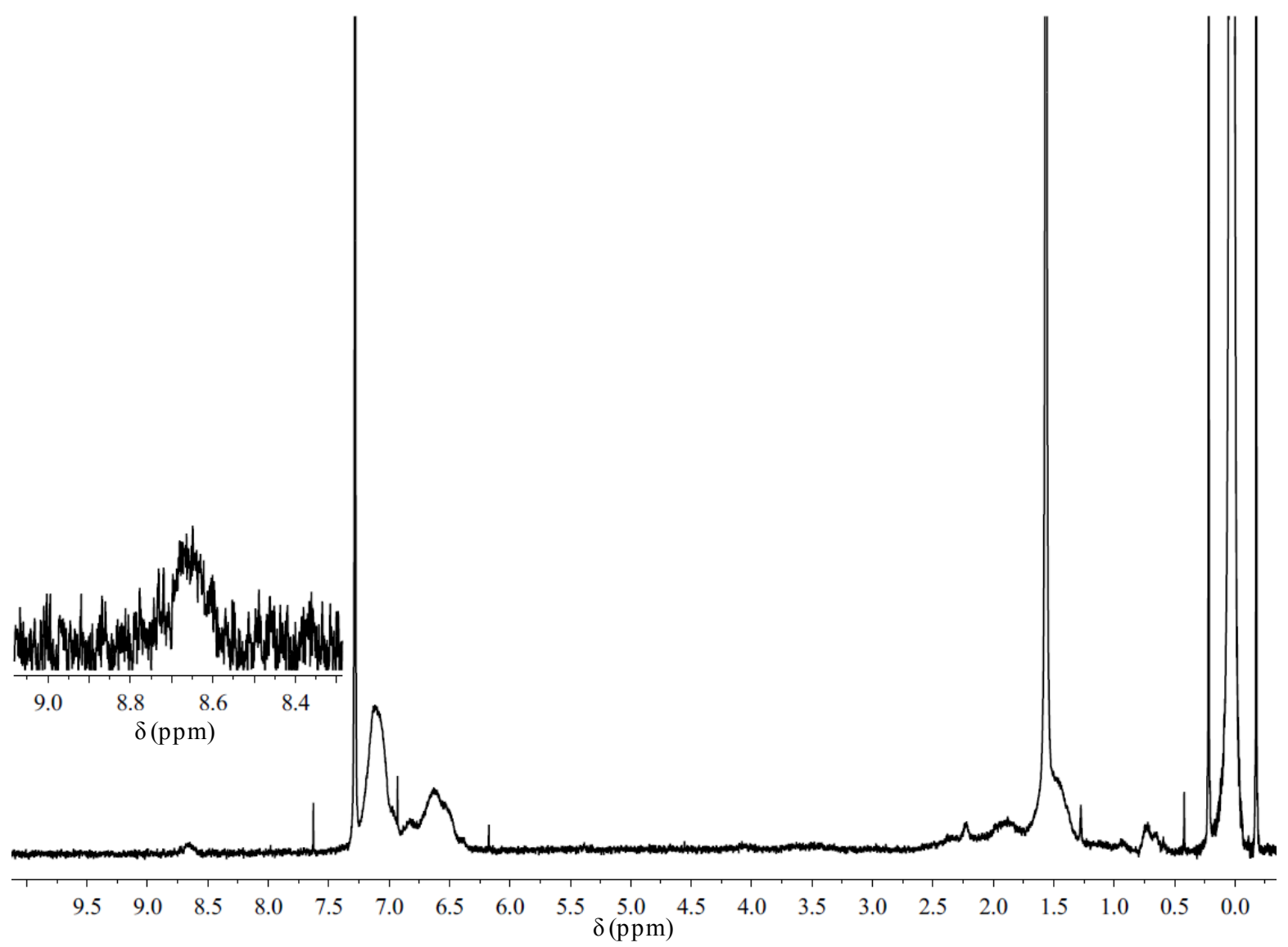

Figure A 3.2 ${ }^{1} \mathrm{HNMR}$ spectra of PS-PTCDI-PS in $\mathrm{CDCl}_{3}$. 
Appendix $4{ }^{1}$ HNMR Spectrum of Propyl amine indicating the purity of the solvent

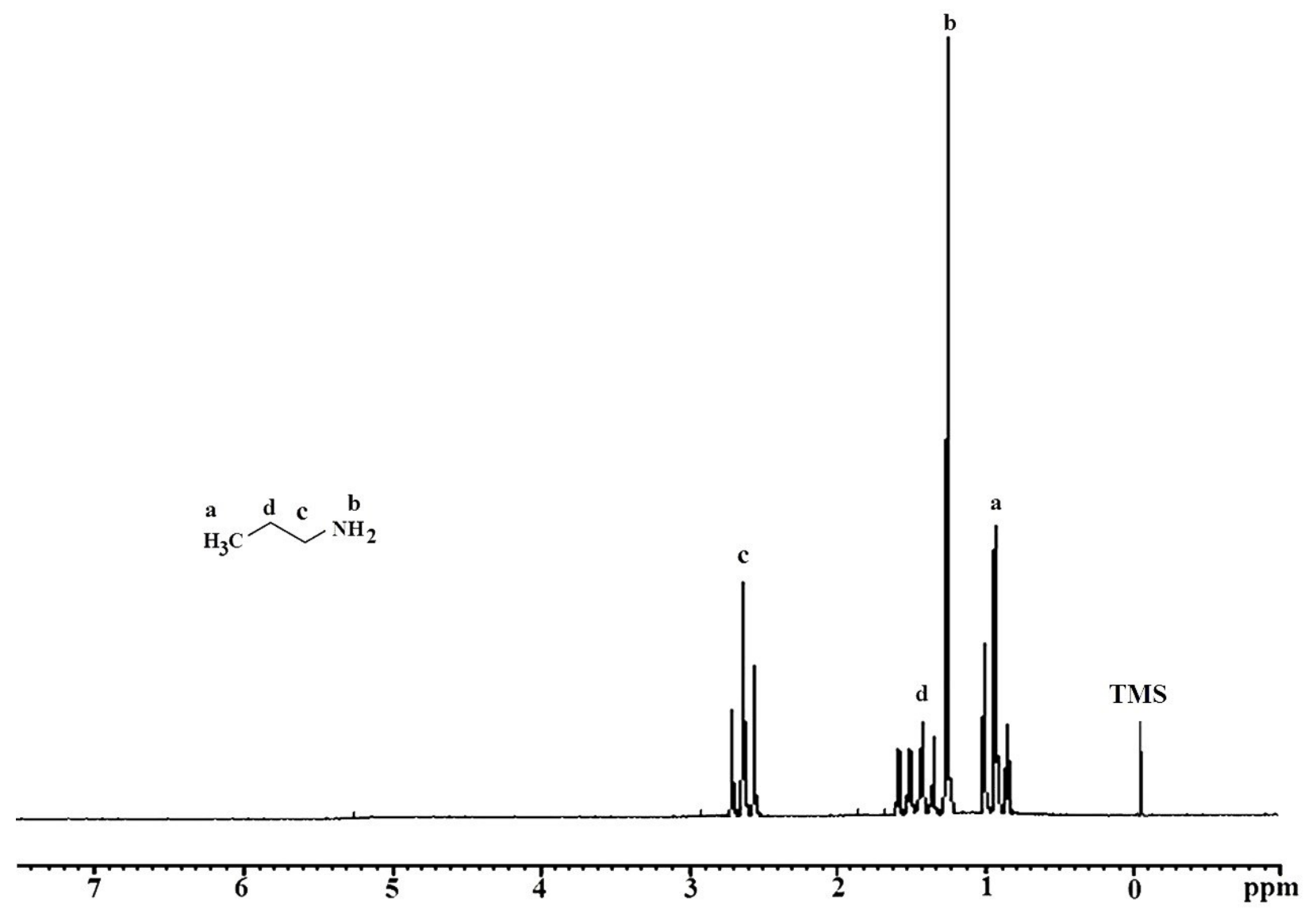

